\title{
The Reaction of 2-Aroylvinylcinnamaldehydes with Aromatic Aldehydes
}

\section{by Dual Catalysis with a Chiral N-Heterocyclic Carbene and a Lewis Acid:}

\section{Enantioselective Construction of Tetrahydroindeno[1,2-c]furan-1-ones}

\author{
Gang Wang, Zhan-Yong Wang, Shuang-Shuo Niu, Yin-Rao and Ying Cheng* \\ College of Chemistry, Beijing Normal University, Beijing 100875, China
}

E-mail: ycheng2@bnu.edu.cn

\section{Table of contents:}

1. X-ray structure of $\mathbf{9 h}$

$\mathrm{pS} 1-\mathrm{S} 2$

2. NMR spectra of major products $4,9,11$, and byproducts $6 \mathbf{a}, \mathbf{6 h}, \mathbf{7 a}$ $\mathrm{pS} 3-\mathrm{S} 80$

3. HPLC spectra of major products $4,9, \mathbf{1 1}$, and byproducts $\mathbf{6 a}, \mathbf{6 h}$. .pS81-S118

Single-crystal diffraction intensity data of the compound $\mathbf{9 h}$ was collected on a Bruker Smart Apex IICCD diffractometer equipped with graphite monochromatized Mo-K $\alpha$ radiation $(\lambda=0.71073 \AA)$ at $100 \mathrm{~K}$. Empirical absorption corrections was applied to the intensities using the SADABS program. The structure was solved by the program SHELXS and refined with the program SHELXL. The disordered solvent molecules were removed with the SQUEEZE routine in PLATON, and the solvent-free model was employed for the final refinement. The crystal data and structure refinement results for compound $\mathbf{9 h}$ are listed in the Table S1.

Table S1. Crystal data and structure refinement results for compound $\mathbf{9 h}{ }^{a}$

\begin{tabular}{|l|l|}
\hline Formula & $\mathrm{C} 33 \mathrm{H} 25 \mathrm{Br} \mathrm{O} 3$ \\
\hline Formula weight & 549.44 \\
\hline Temperature $(\mathrm{K})$ & $100(2)$ \\
\hline Wavelength $(\AA)$ & 0.71073 \\
\hline Crystal system & Monoclinic \\
\hline Space group & $\mathrm{C} 2$ \\
\hline$a(\AA)$ & $\mathrm{a}=28.515(18)$ \\
\hline$b(\AA)$ & $\mathrm{b}=7.530(5)$ \\
\hline$c(\AA)$ & $\mathrm{c}=15.194(10)$ \\
\hline$\alpha\left(^{\circ}\right)$ & $90^{\circ}$ \\
\hline$\beta\left(^{\circ}\right)$ & $115.987(10)^{\circ}$ \\
\hline$\gamma\left({ }^{\circ}\right)$ & $90^{\circ}$ \\
\hline Volume $\left.^{\circ} \AA^{3}\right)$ & $2933(3)$ \\
\hline
\end{tabular}




\begin{tabular}{|l|l|}
\hline $\mathrm{Z}$ & 4 \\
\hline Calculated density $\left(\mathrm{Mg} / \mathrm{m}^{3}\right)$ & 1.244 \\
\hline Absorption coefficient $\left(\mathrm{mm}^{-1}\right)$ & 1.431 \\
\hline $\mathrm{F}(000)$ & 1128 \\
\hline Crystal size $(\mathrm{mm})$ & $0.350 \mathrm{x} 0.150 \mathrm{x} 0.100$ \\
\hline Theta range for data collection $\left(^{\circ}\right)$ & 1.589 to 27.568 \\
\hline Reflections collected & 9785 \\
\hline Independent reflections & 5892 \\
\hline Completeness to theta $=25.242^{\circ}$ & $99.9 \%$ \\
\hline Absorption correction & Semi-empirical from equivalents \\
\hline Max. and min. transmission & 0.75 and 0.50 \\
\hline Refinement method & Full-matrix least-squares on $\mathrm{F}^{2}$ \\
\hline Data / restraints / parameters & $5892 / 13 / 335$ \\
\hline Goodness-of-fit on F ${ }^{2}$ & 1.059 \\
\hline Final R indices [I $>2$ sigma $(\mathrm{I})]$ & $\mathrm{R} 1=0.0672, \mathrm{wR} 2=0.1786$ \\
\hline R indices (all data) & $\mathrm{R} 1=0.0949, \mathrm{wR} 2=0.1922$ \\
\hline Absolute structure parameter & $0.08(2)$ \\
\hline Largest diff. peak and hole & 0.382 and -1.066 e. $\AA^{-3}$ \\
\hline
\end{tabular}

${ }^{a}$ The data is based on the solvent-free model.

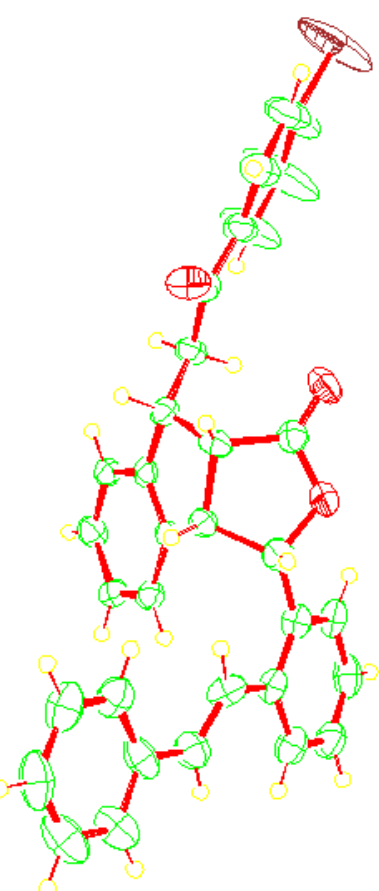

Figure S1. ORTEP drawing of X-ray structure of $\mathbf{9 h}$. The ellipsoid contour probability level is $50 \%$. 


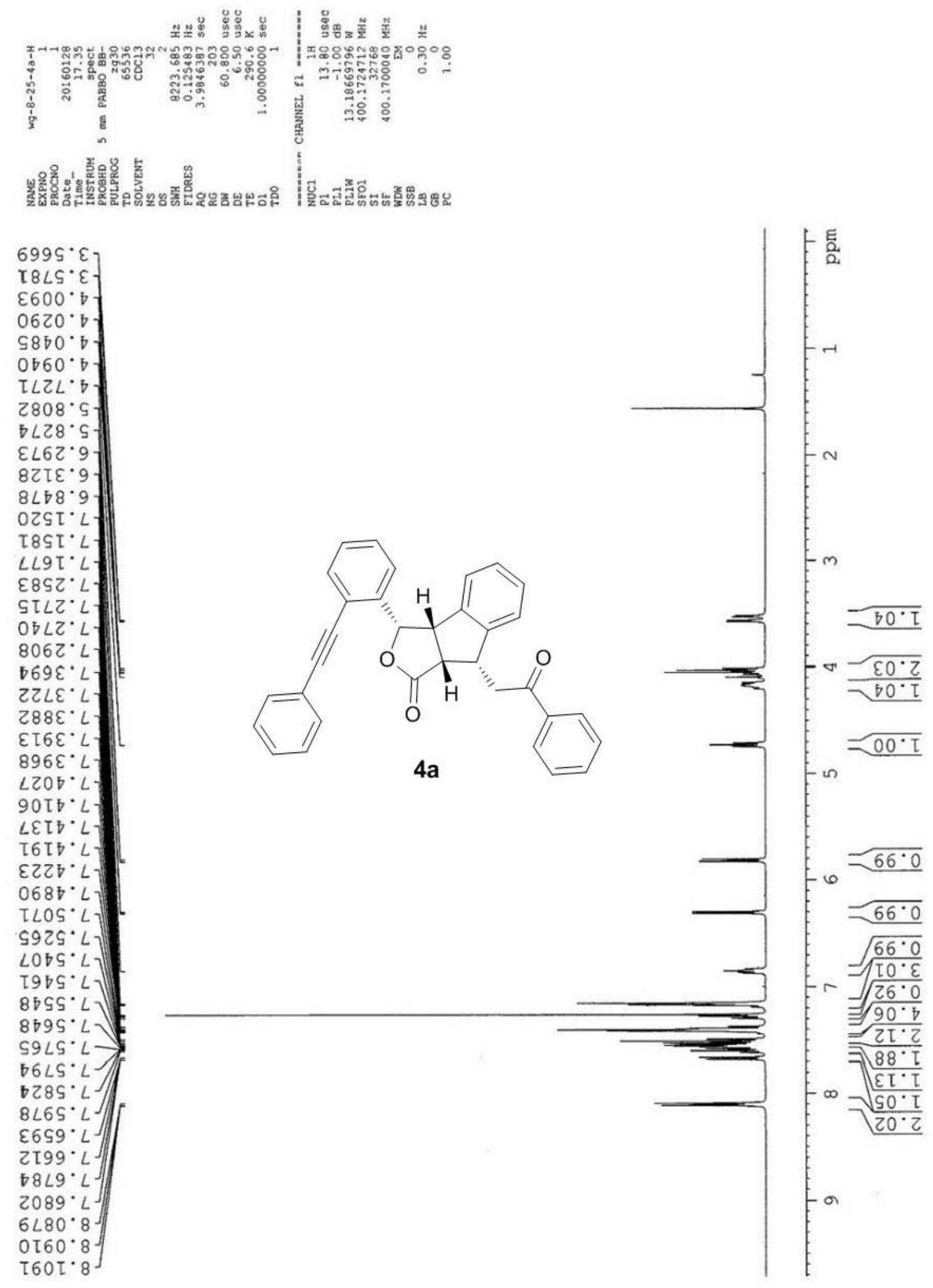



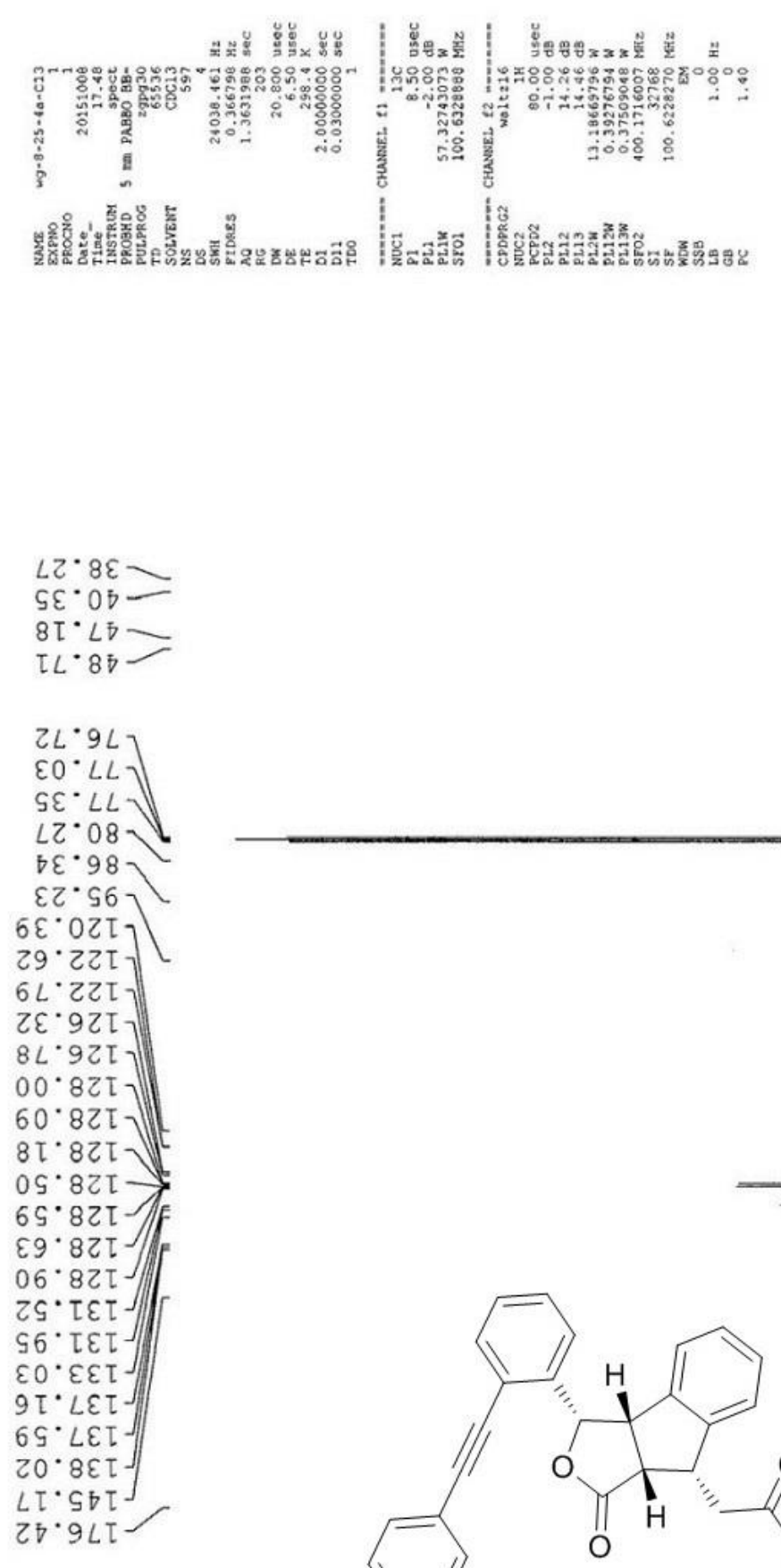

$\varepsilon \varepsilon \cdot 66 \tau-$

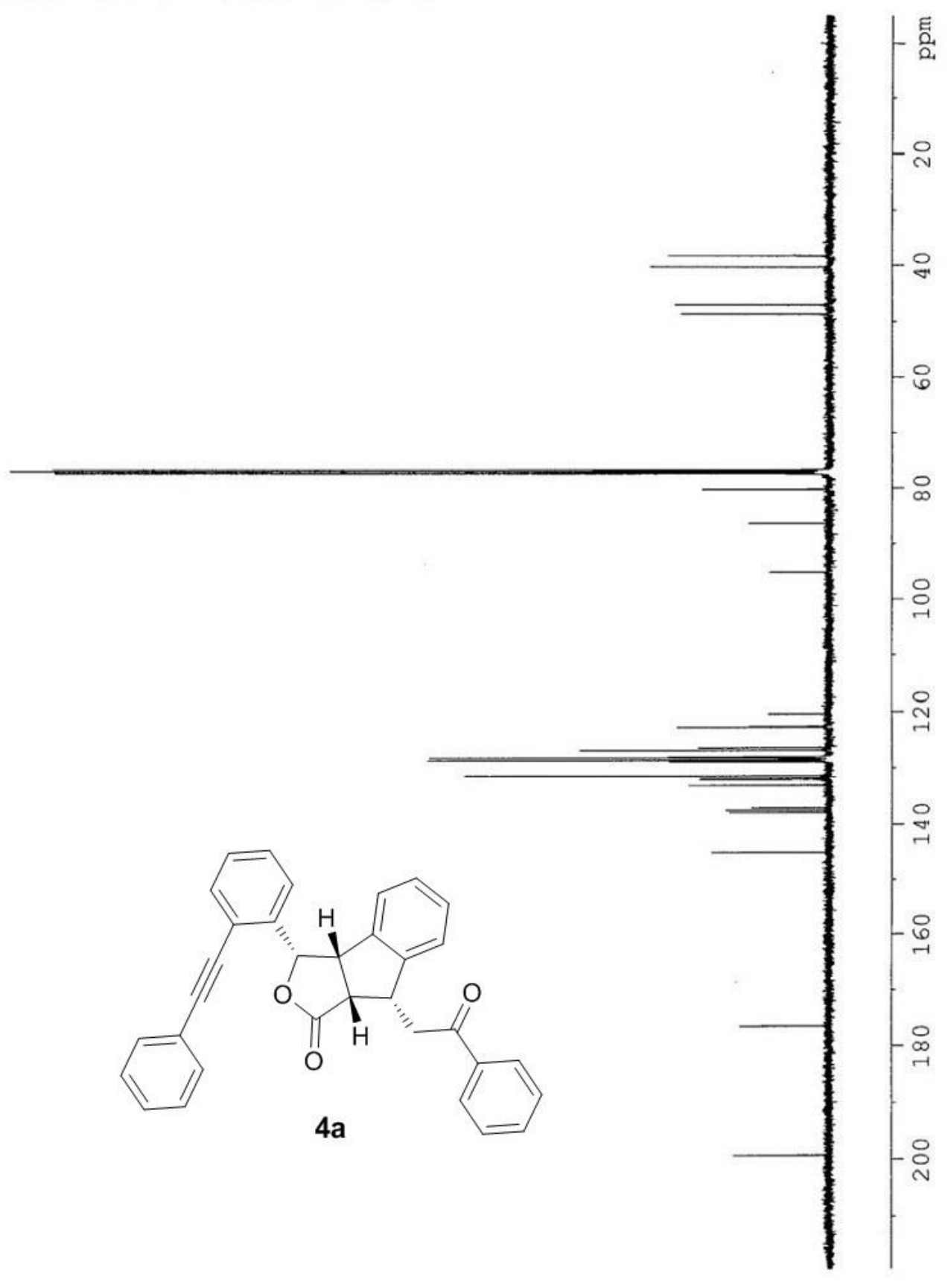




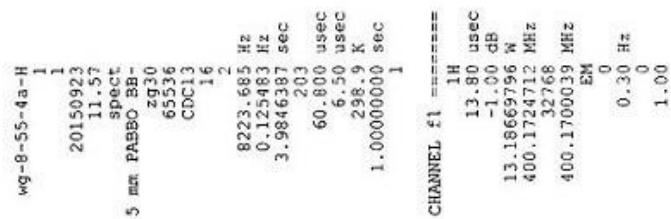

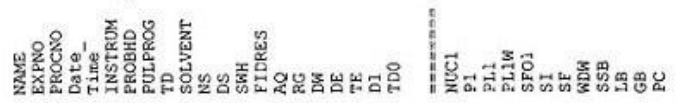
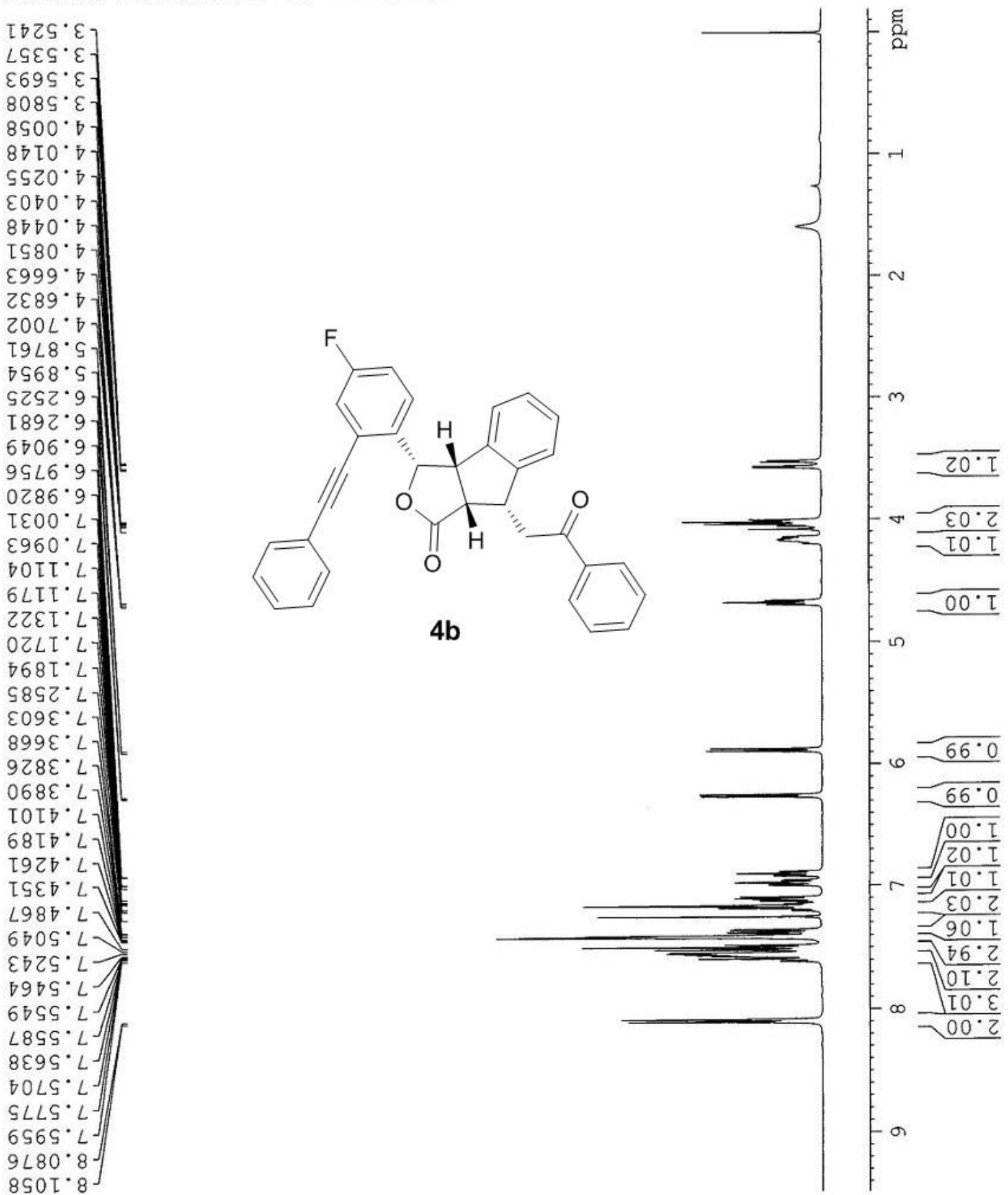


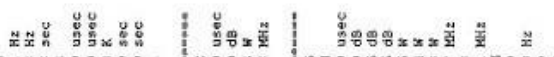
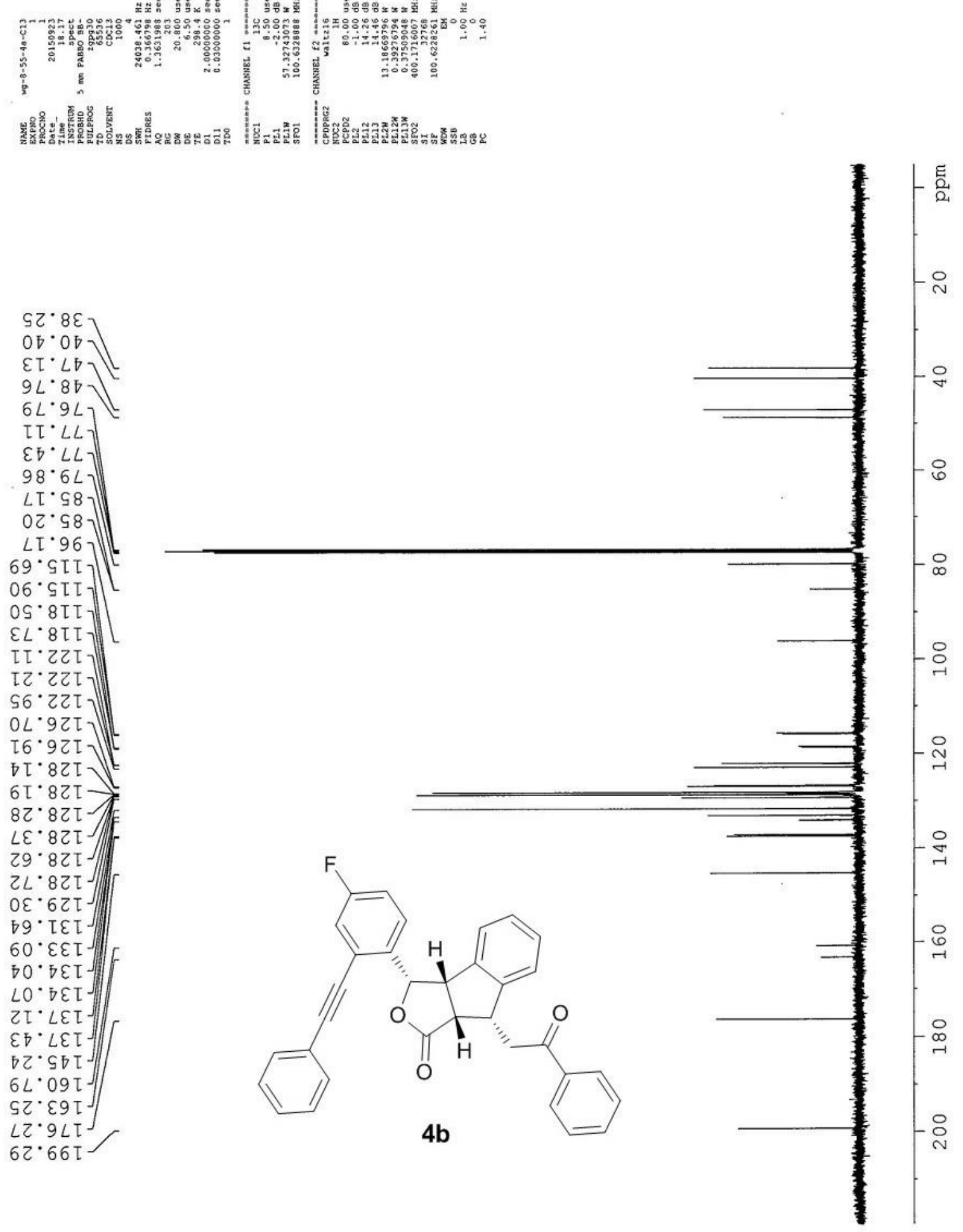

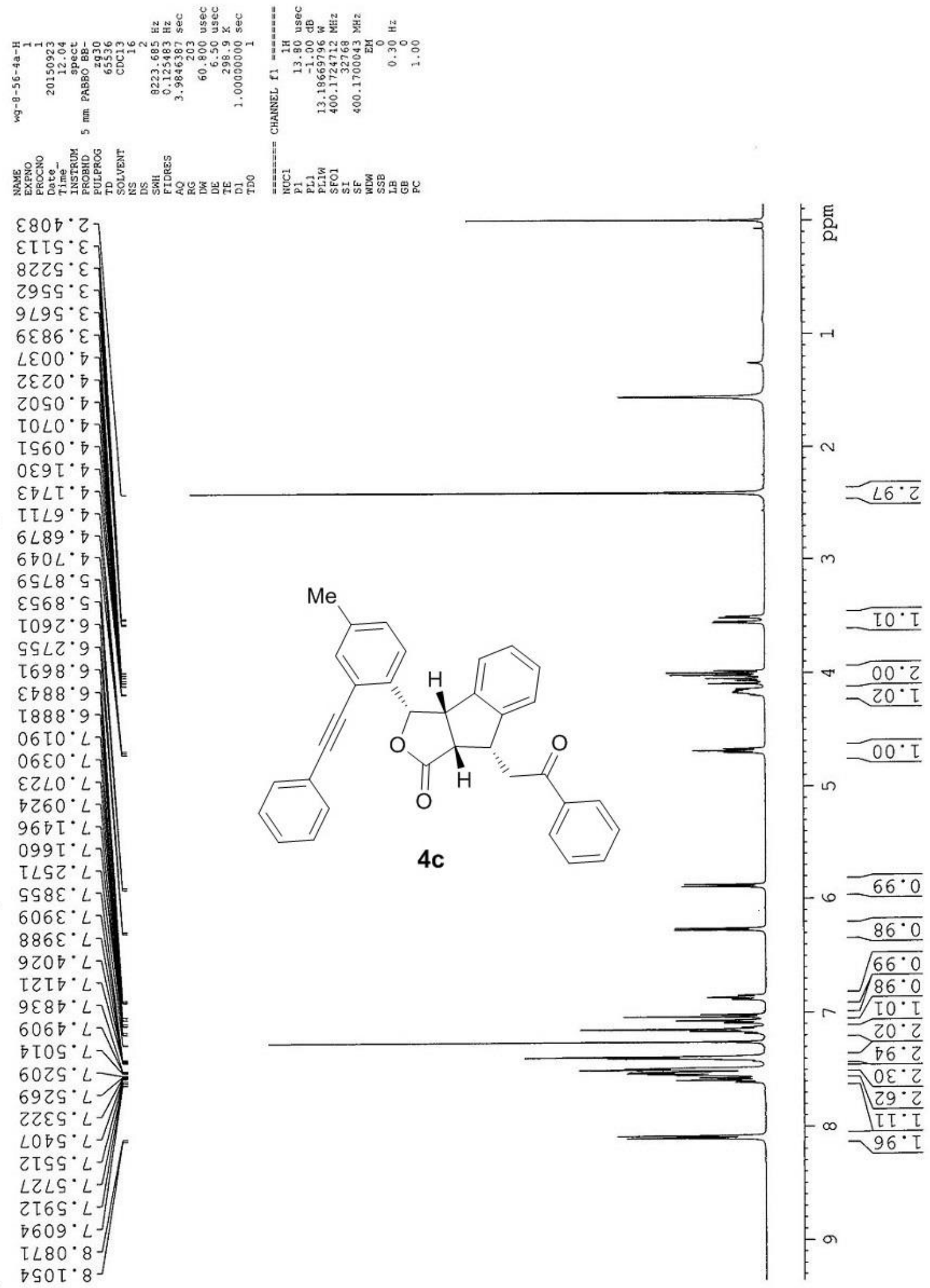


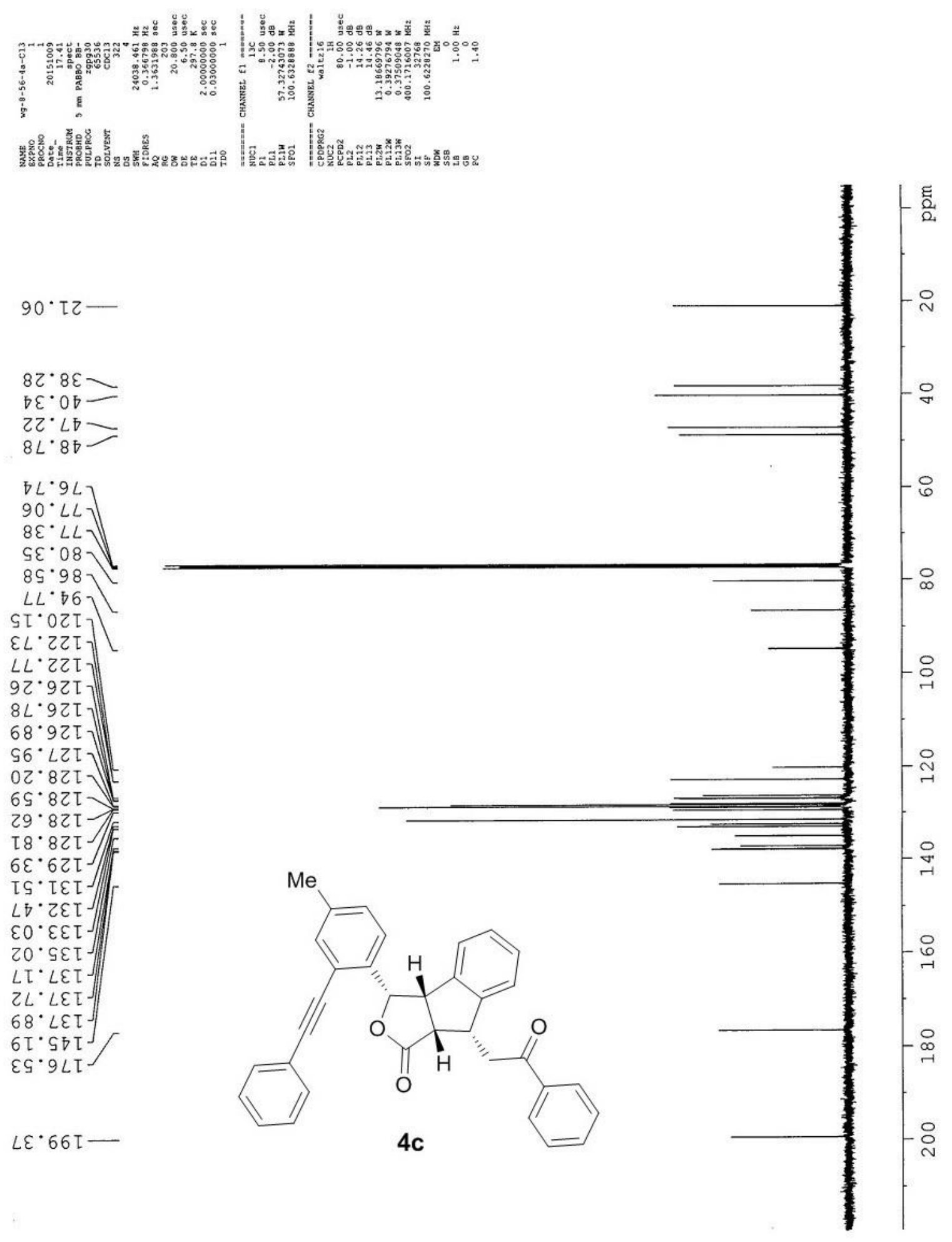




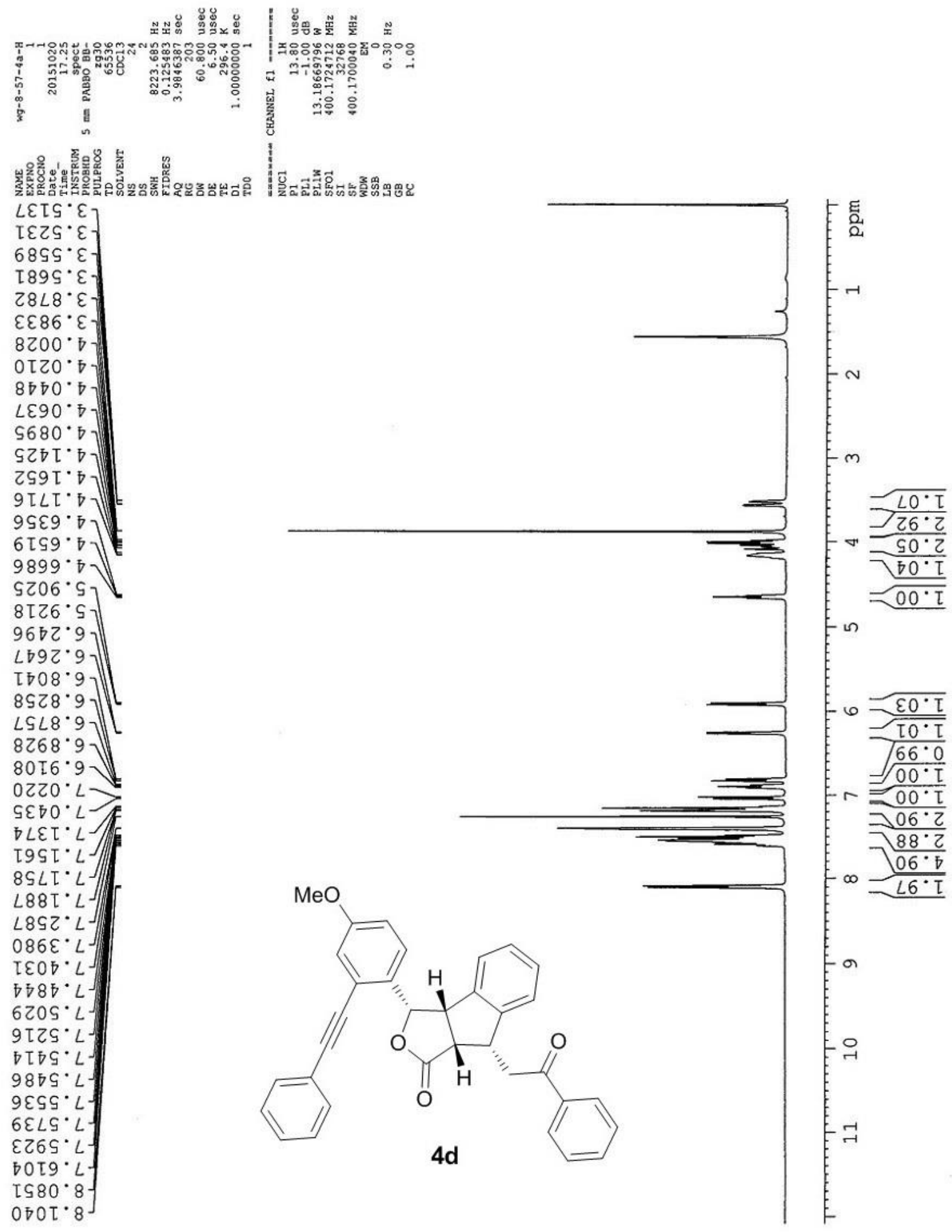




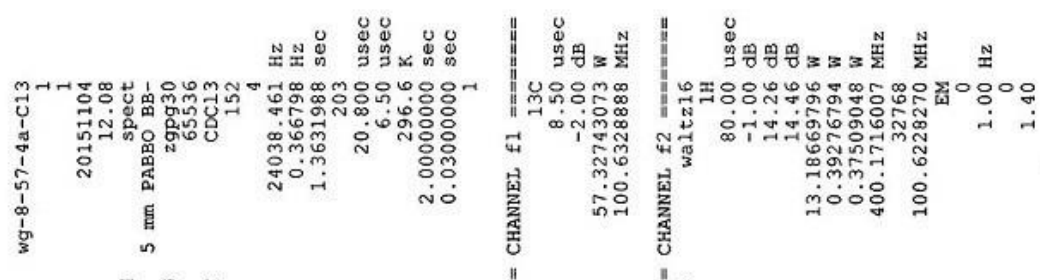

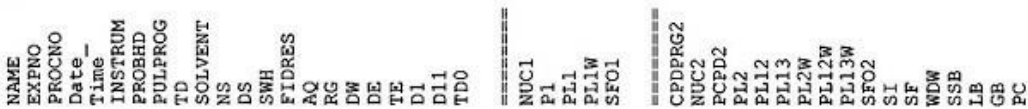

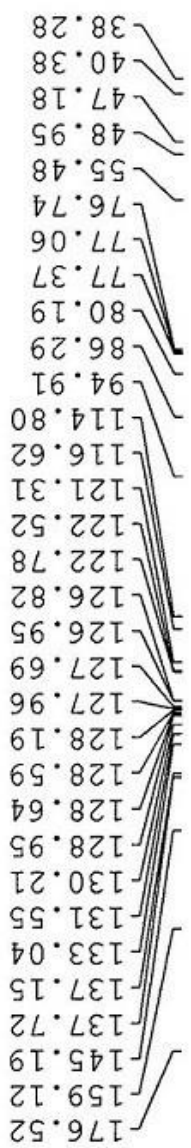

$9 \varepsilon \cdot 66 \tau-$

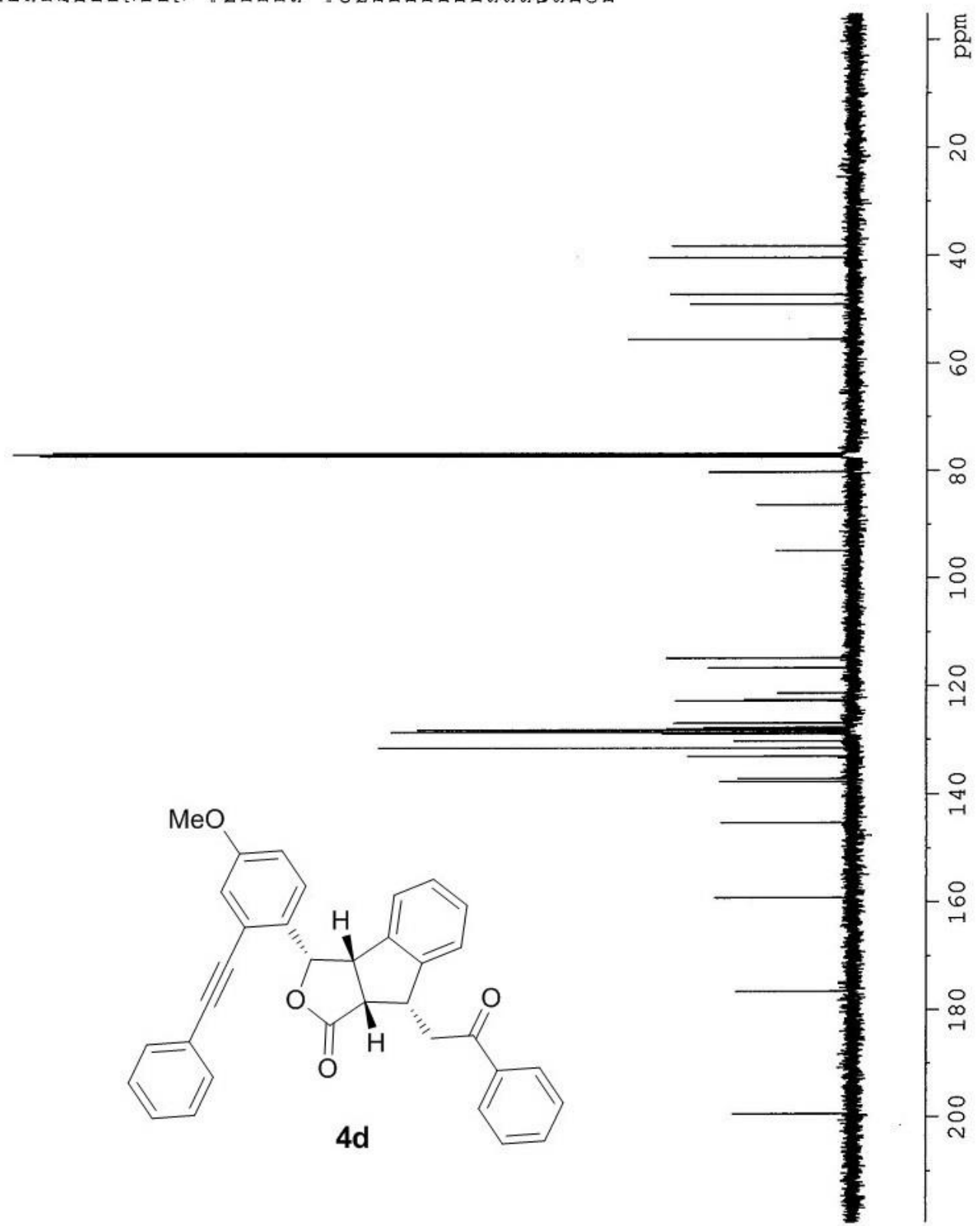




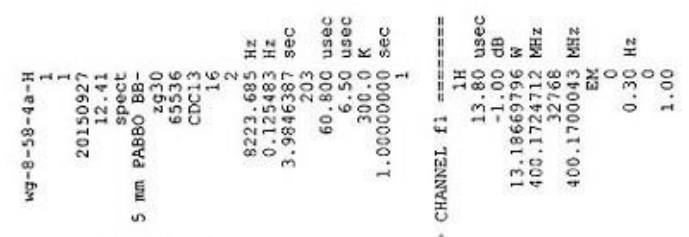

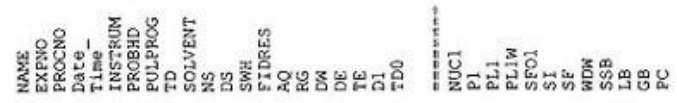
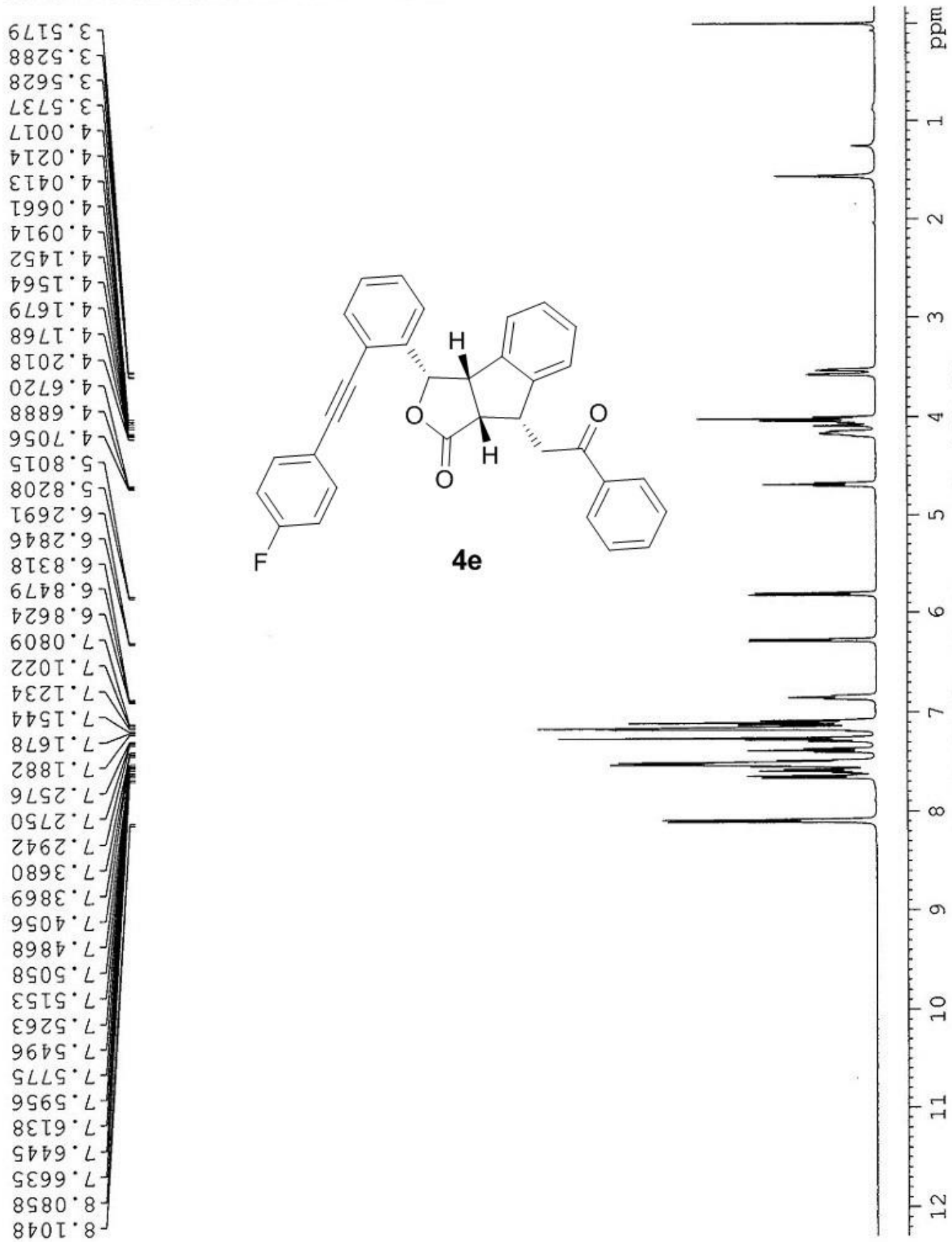

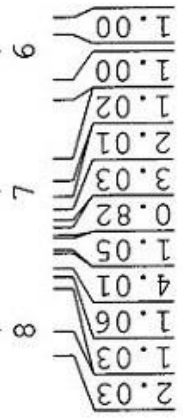

a

E 


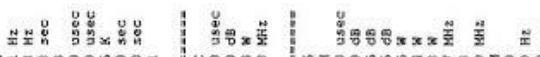
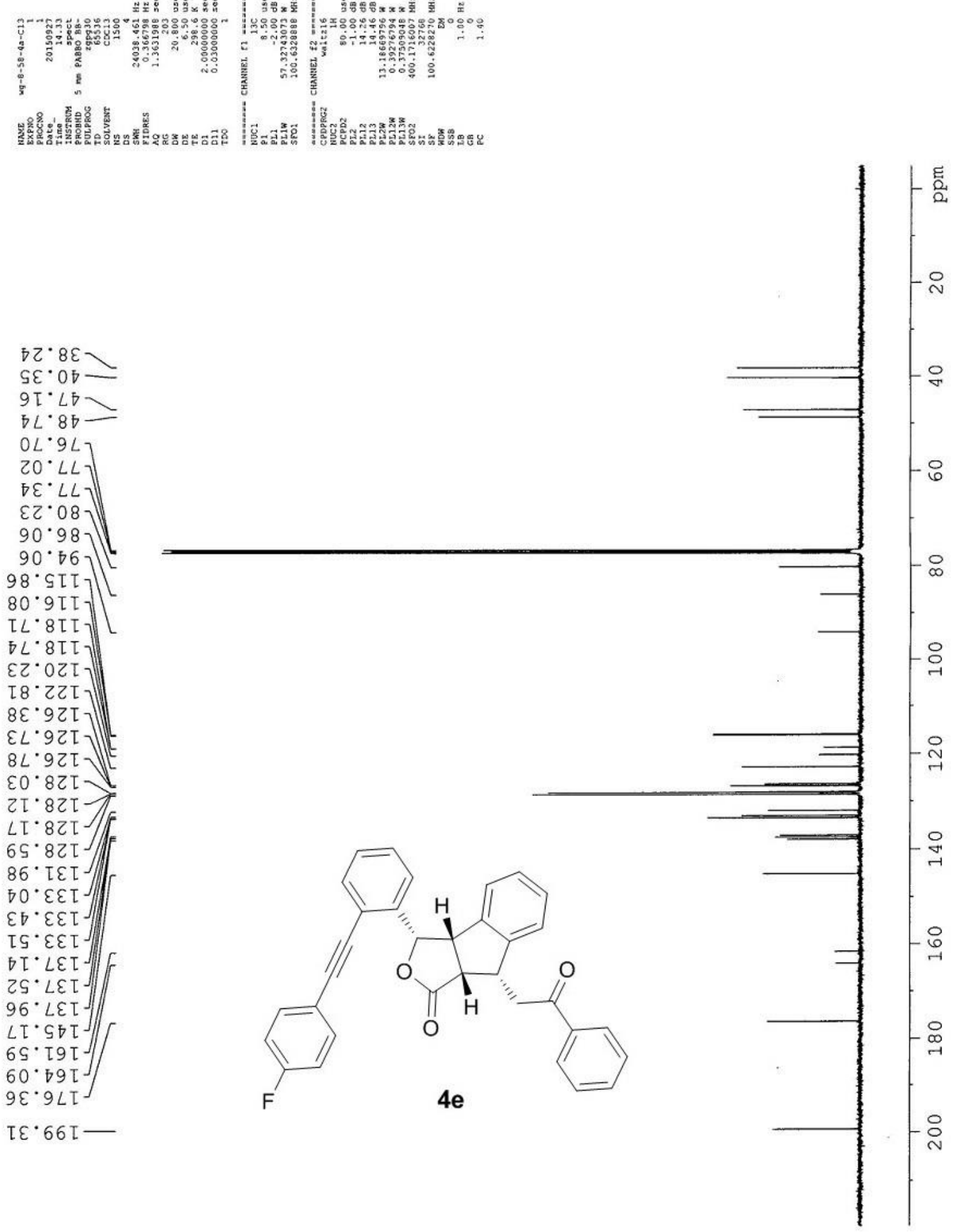


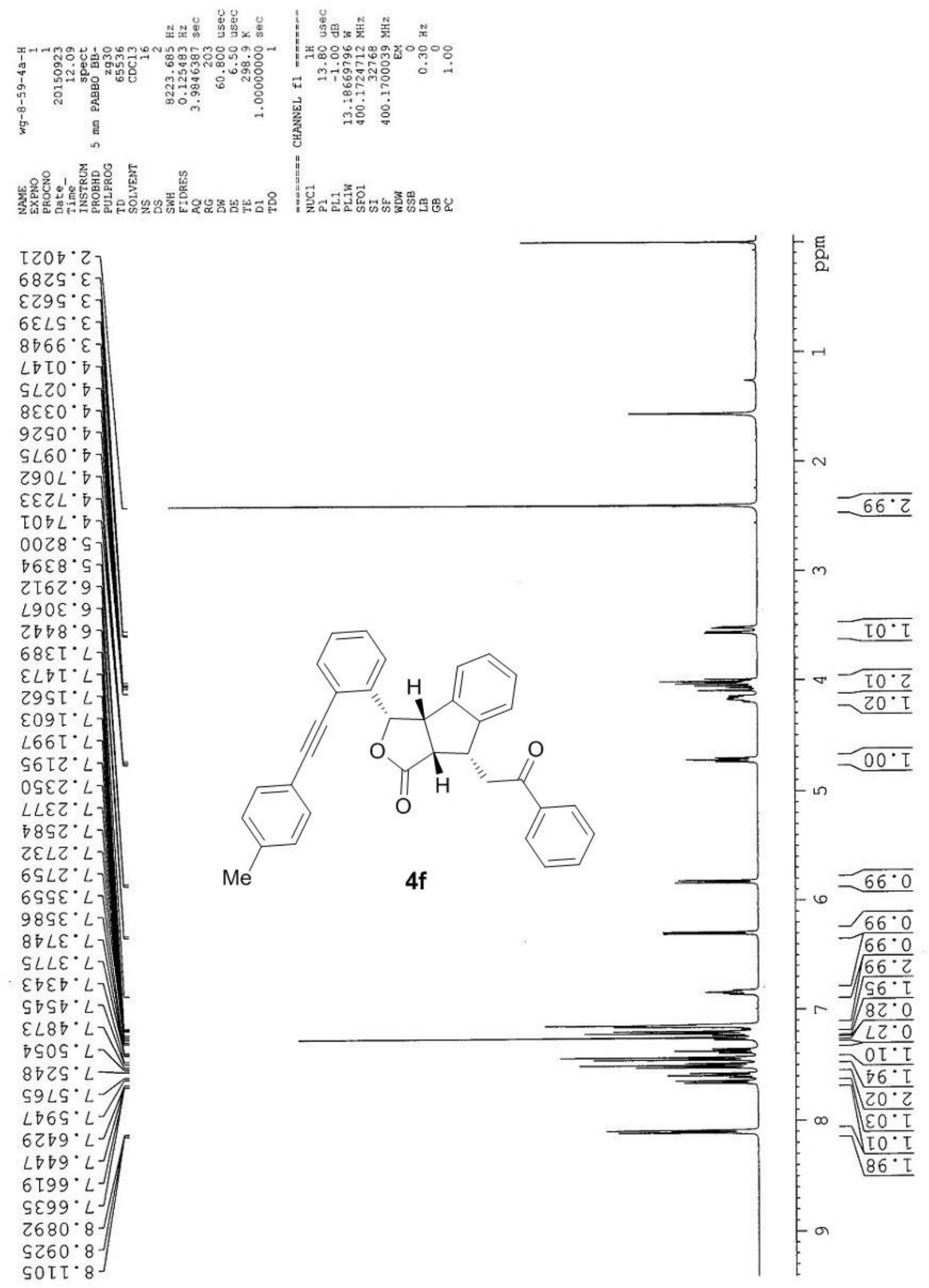




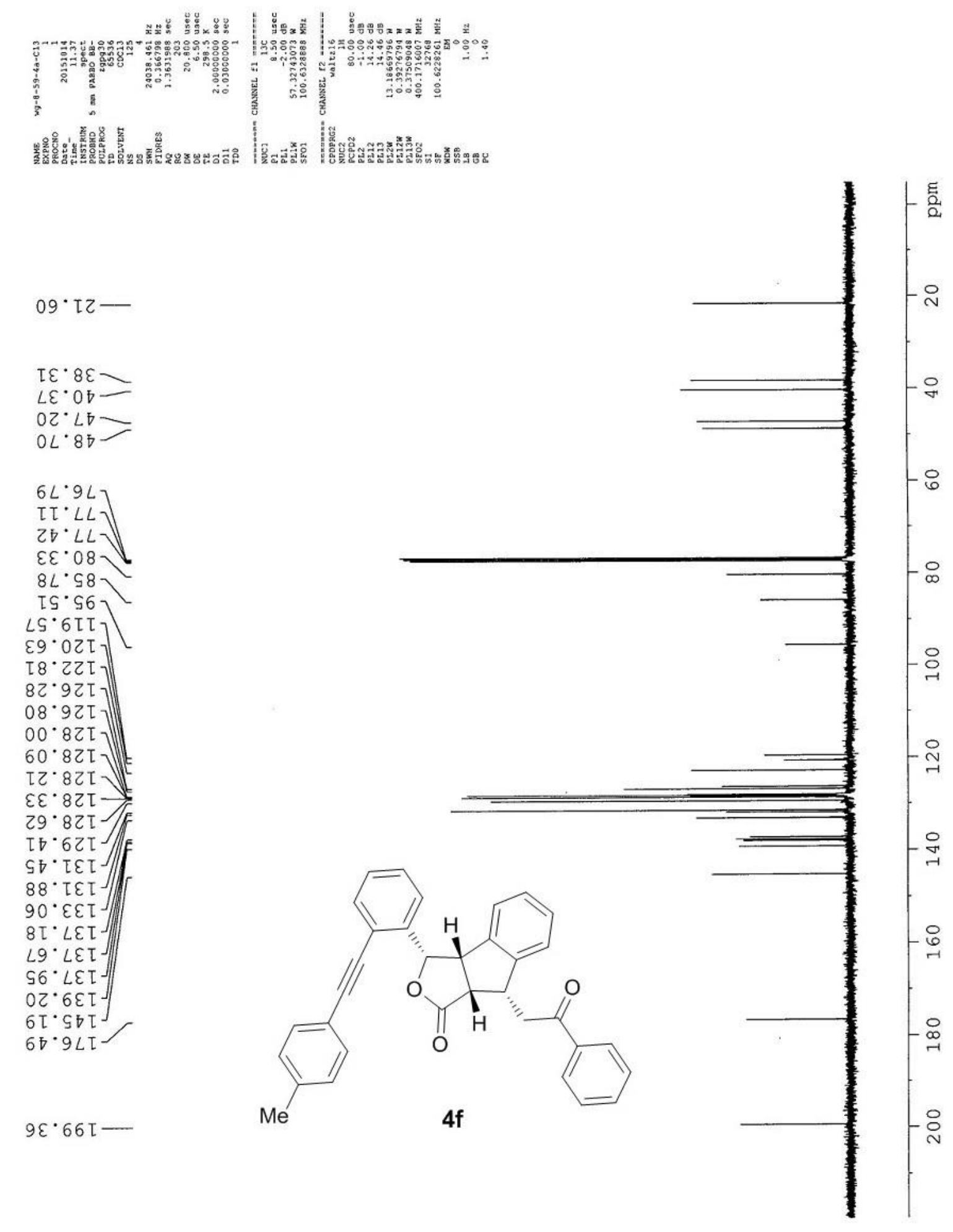



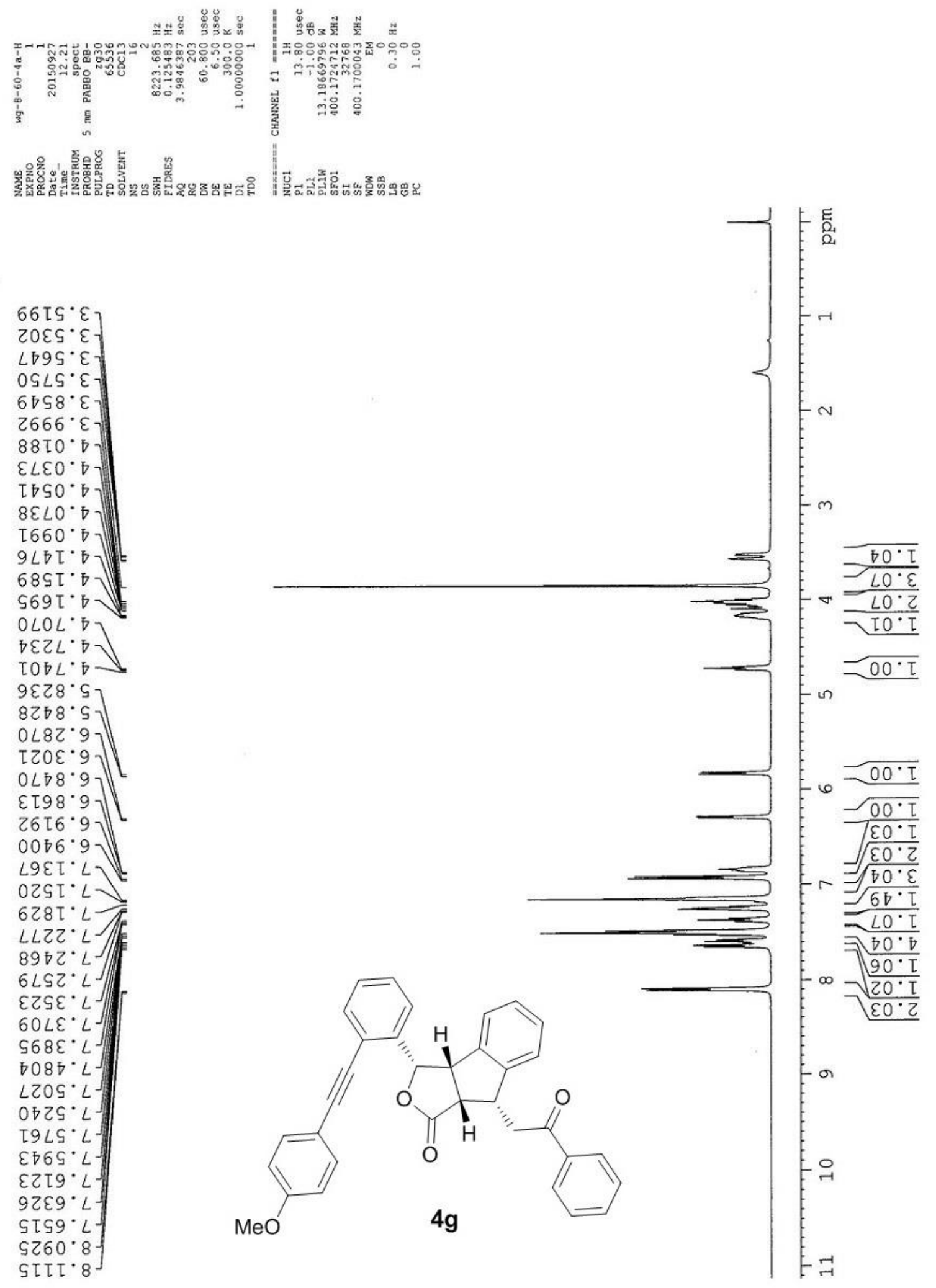

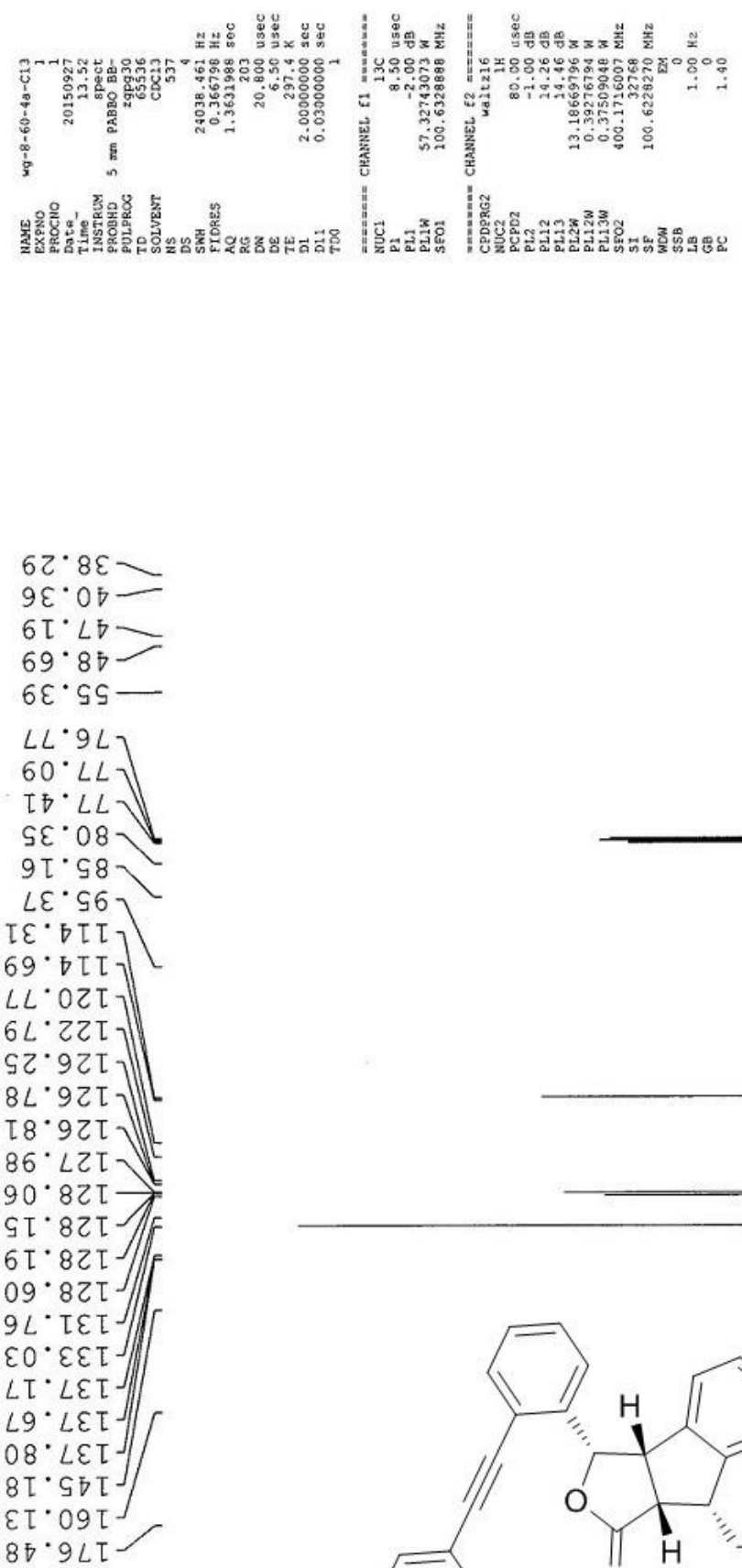

$\varsigma \varepsilon \cdot 66 \mathrm{I}$

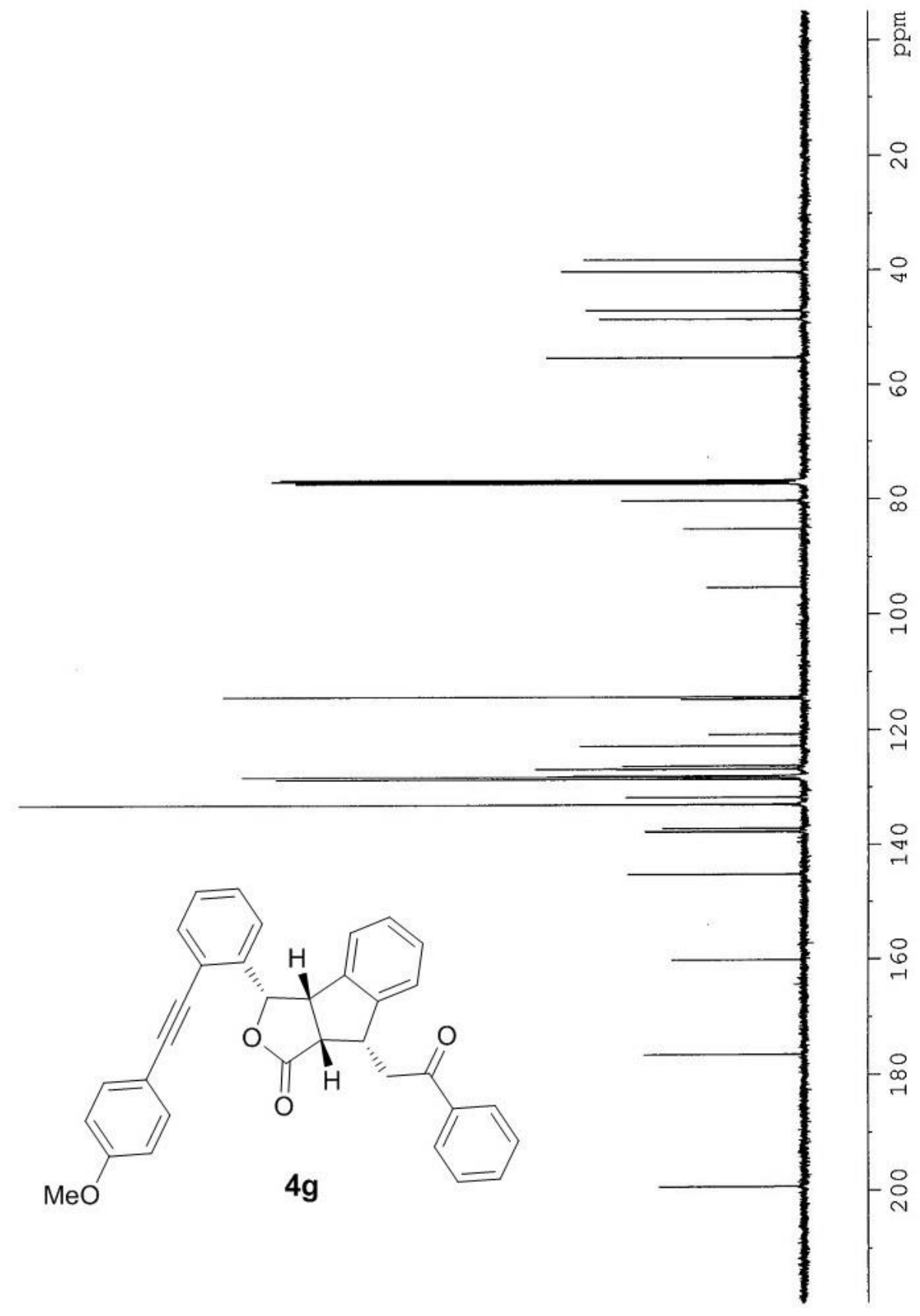




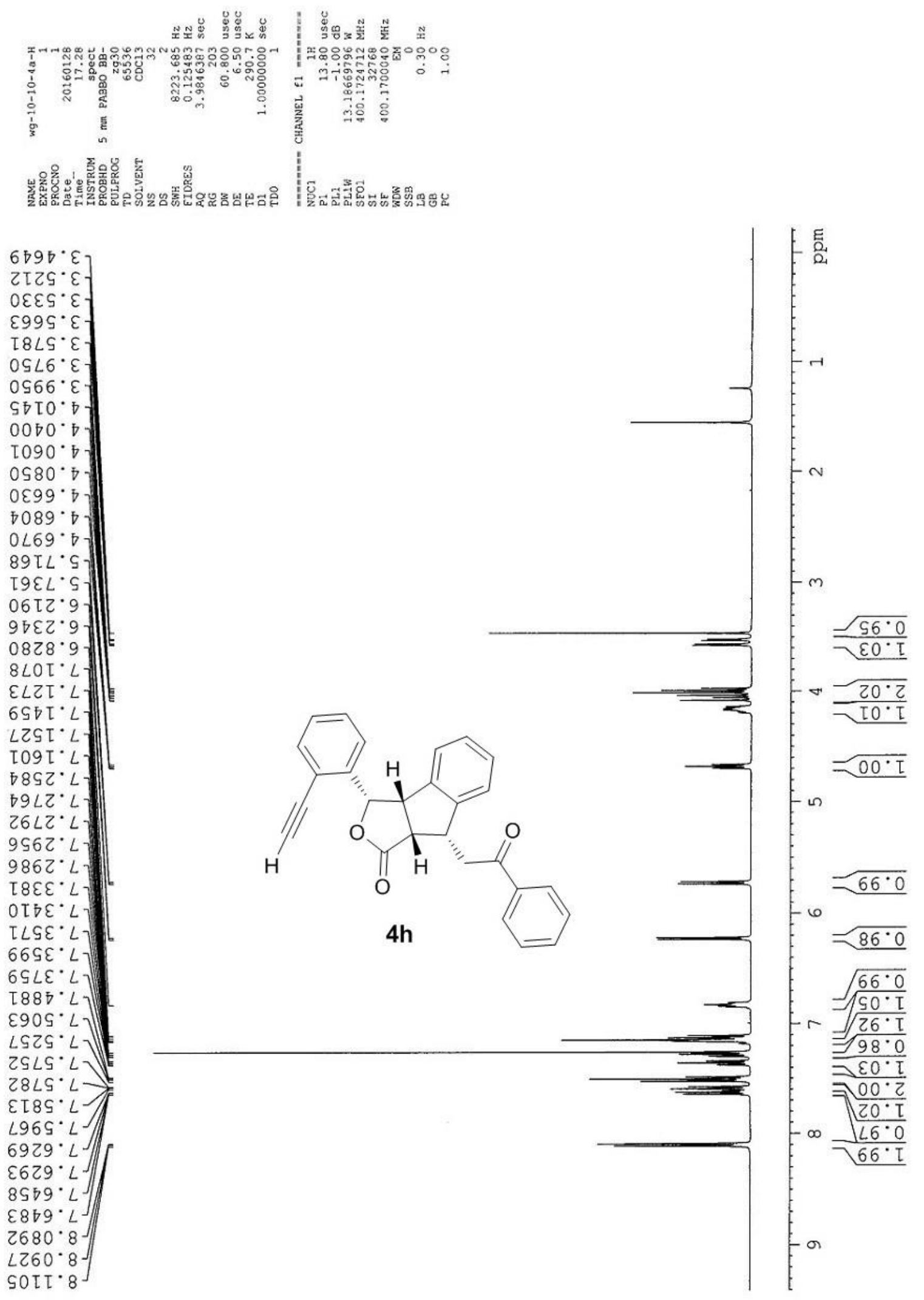



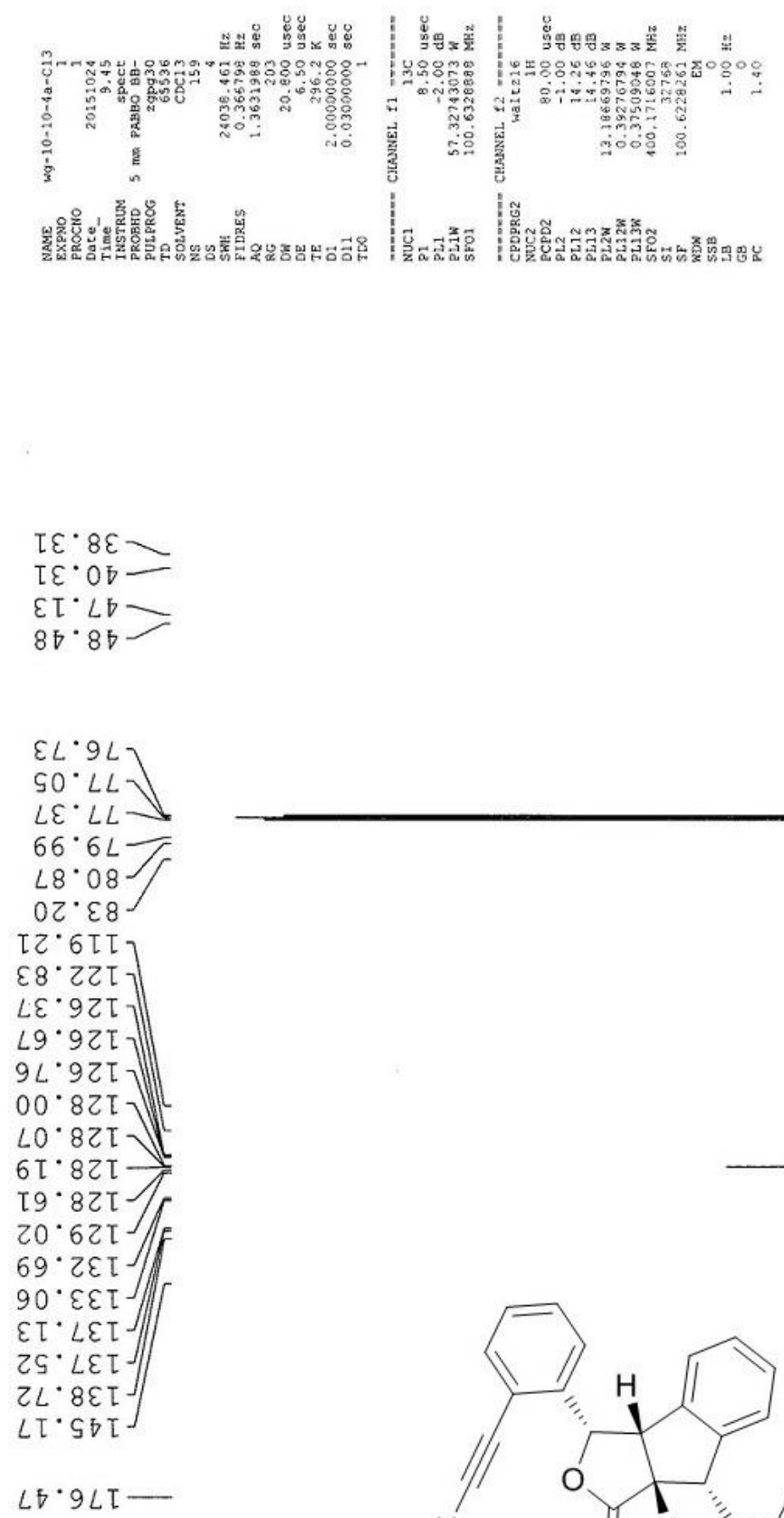

$62 \cdot 66$ I
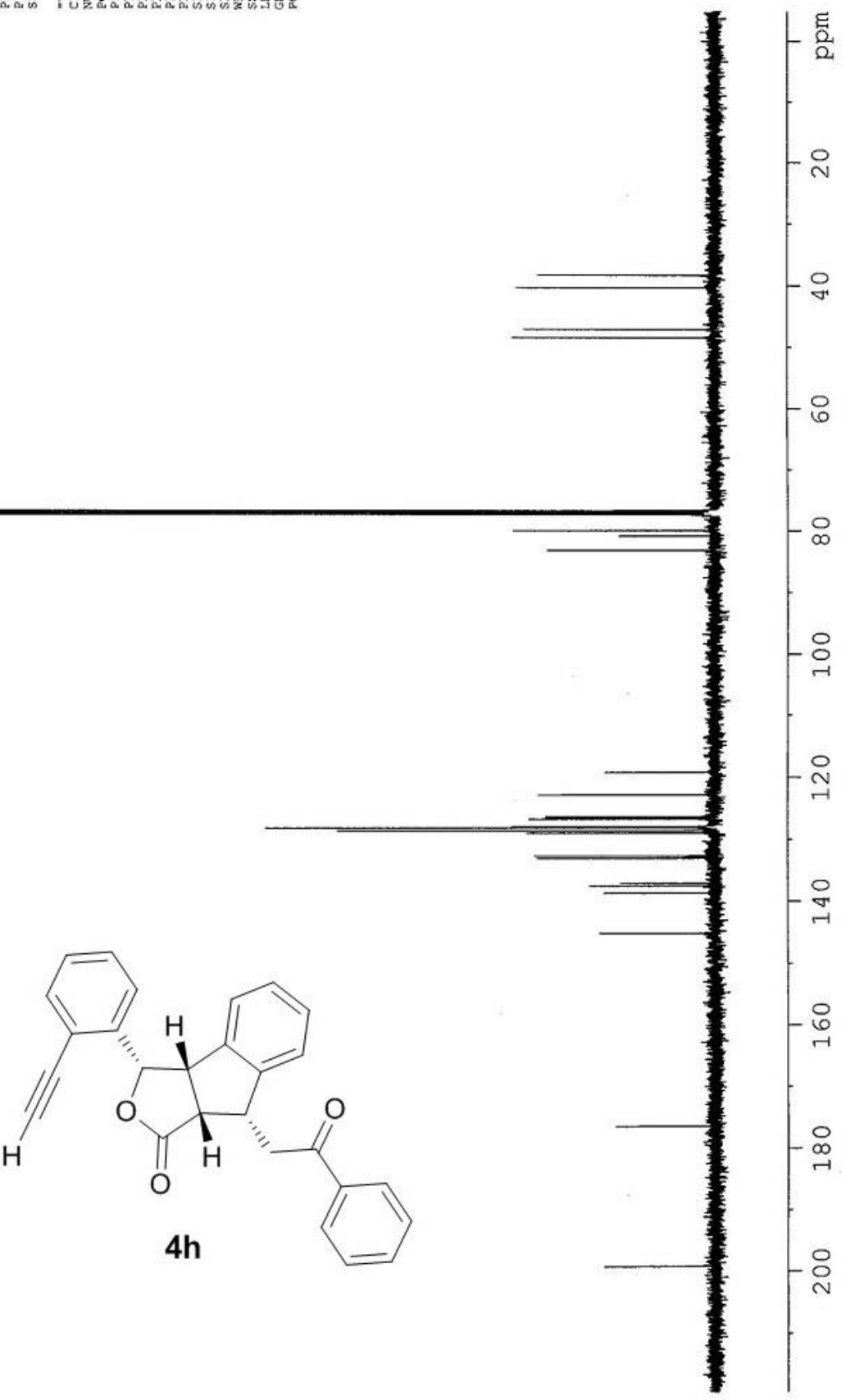


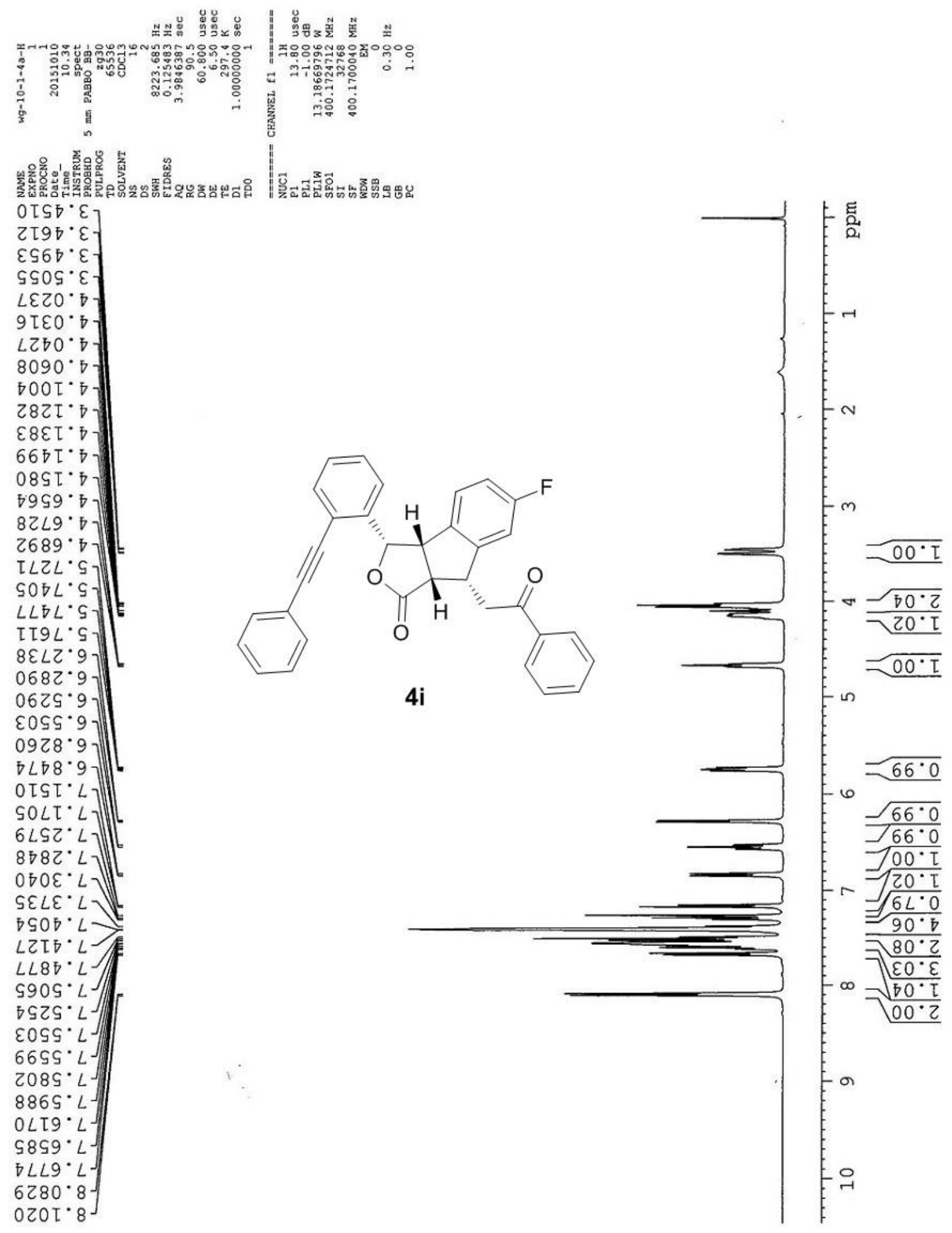




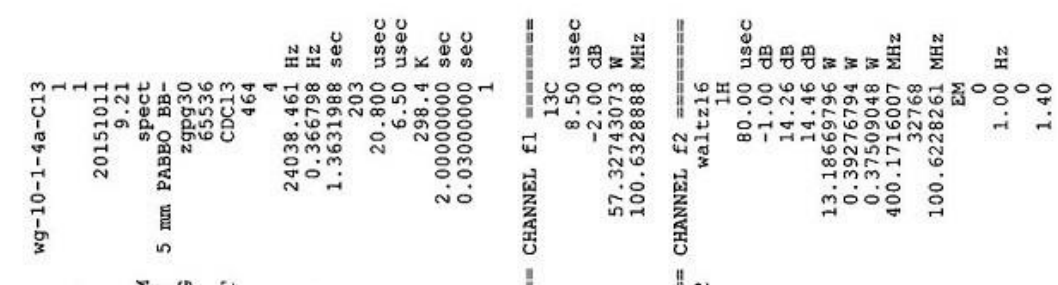

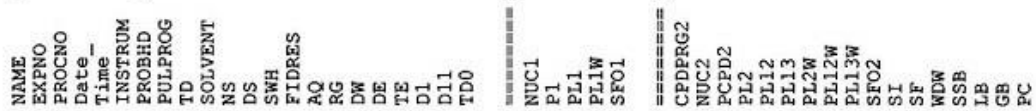
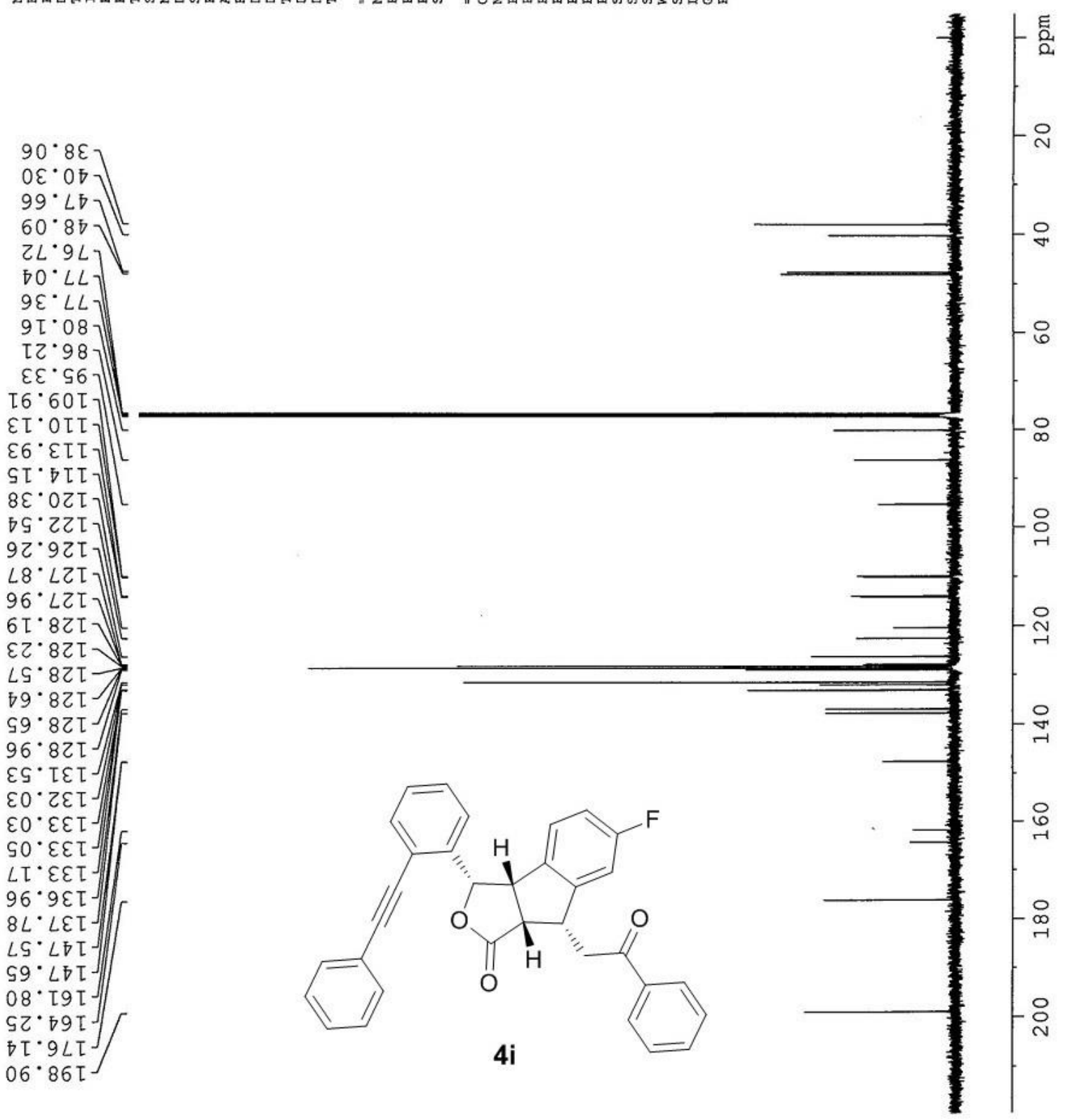


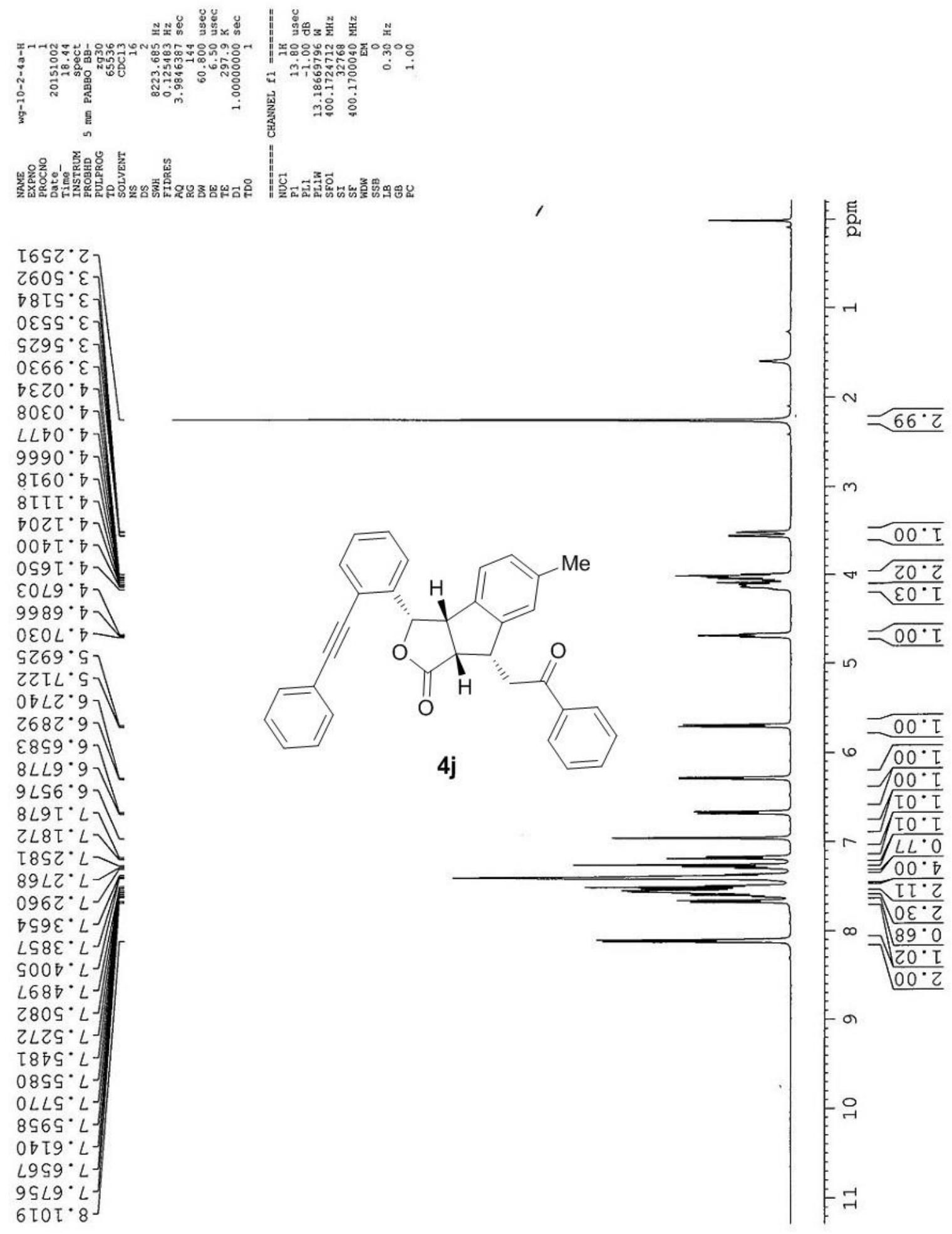




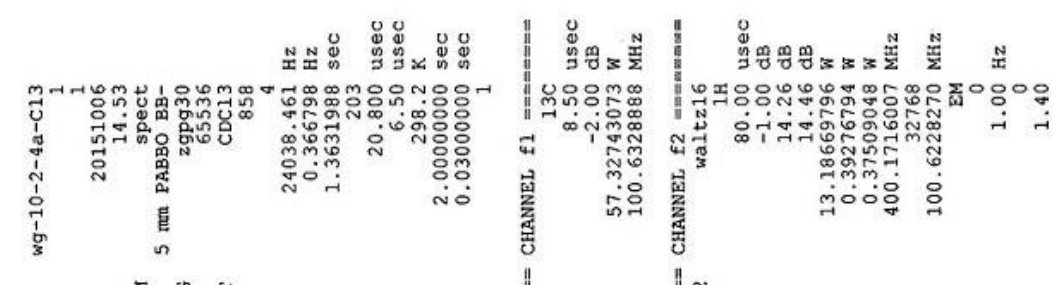

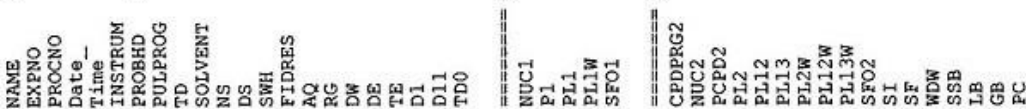

$L Z \cdot I Z=$

$8 Z \cdot 8 \varepsilon=$
$L T \cdot 0 \sigma=$
$\varepsilon \sigma \cdot \angle \sigma=$

$8 \varepsilon \cdot 8 \sigma$

$69 \cdot 9 L$

I0. $L L$

$\left.\begin{array}{l}92 \cdot 08 \\ L \varepsilon \cdot 98\end{array}\right]$

9โ. 56$]$

ธะ.0Z [ ]

$\varepsilon 9^{\circ}$ Zน ]

$6 \varepsilon \cdot \varepsilon ट \tau$

$0 \varepsilon^{\circ} \cdot 9 Z \tau$

ZV.9ZT

$\square L \cdot L Z[]$

$[0.8 Z[1]$

$90^{\circ} 82 \tau$

$\angle S^{\circ} 8 \mathrm{ZL}$

โ9.8Zโ

$98^{\circ} 82$ โ

$0 S^{\circ}$ โ $\tau$ I

โ6. ${ }^{\circ} \varepsilon \tau$

$00^{\circ} \varepsilon \varepsilon \tau$ -

$09^{\circ} \nabla \varepsilon[$

$L I \cdot L E[$

$58^{\circ} L E[$ -

$\mathrm{SI} \cdot 8 \varepsilon[-$

$6 Z \cdot S t[$

$5 \mathrm{~S} \cdot 9 L \tau$

ID. $66 \tau$ -

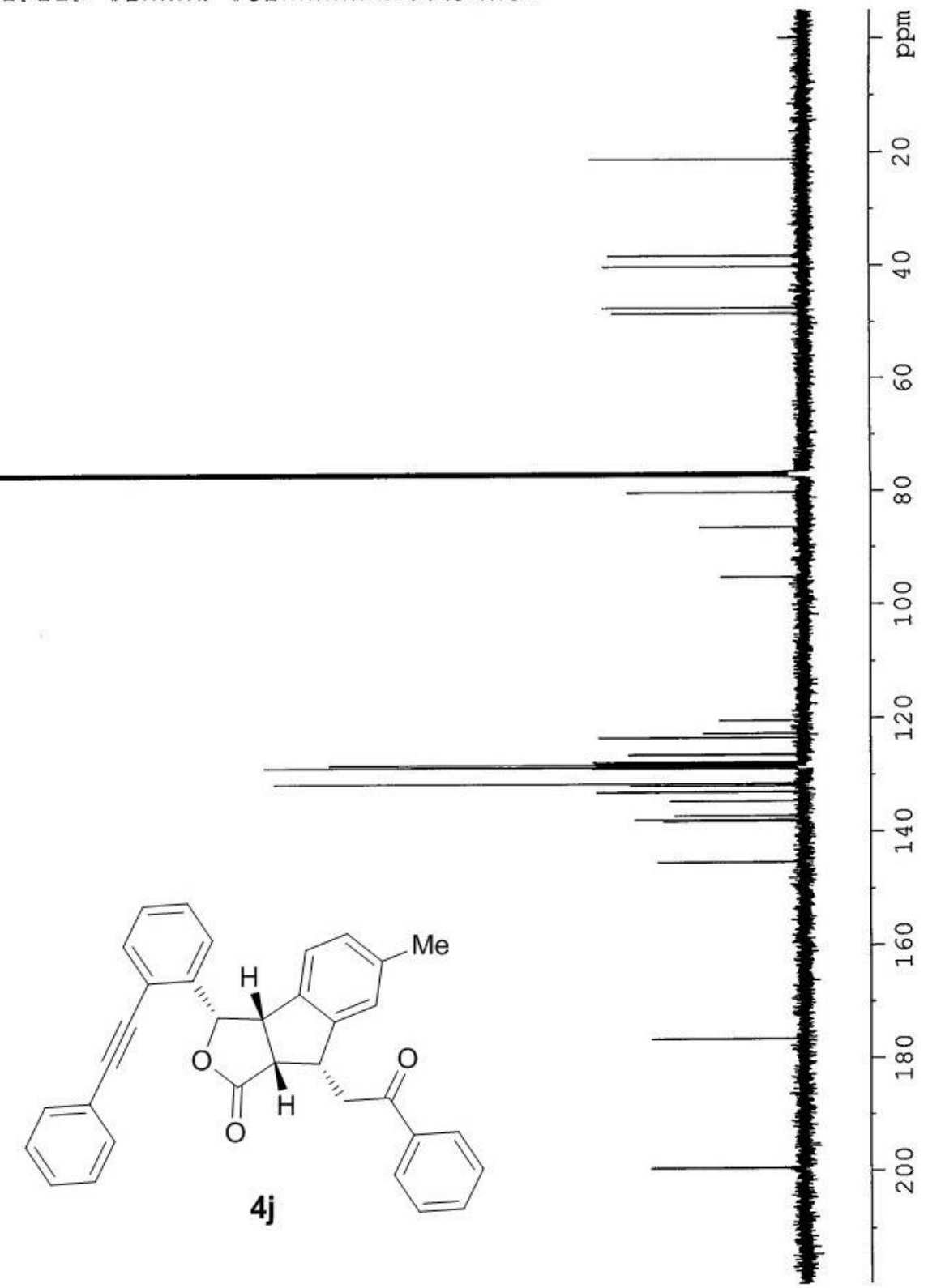




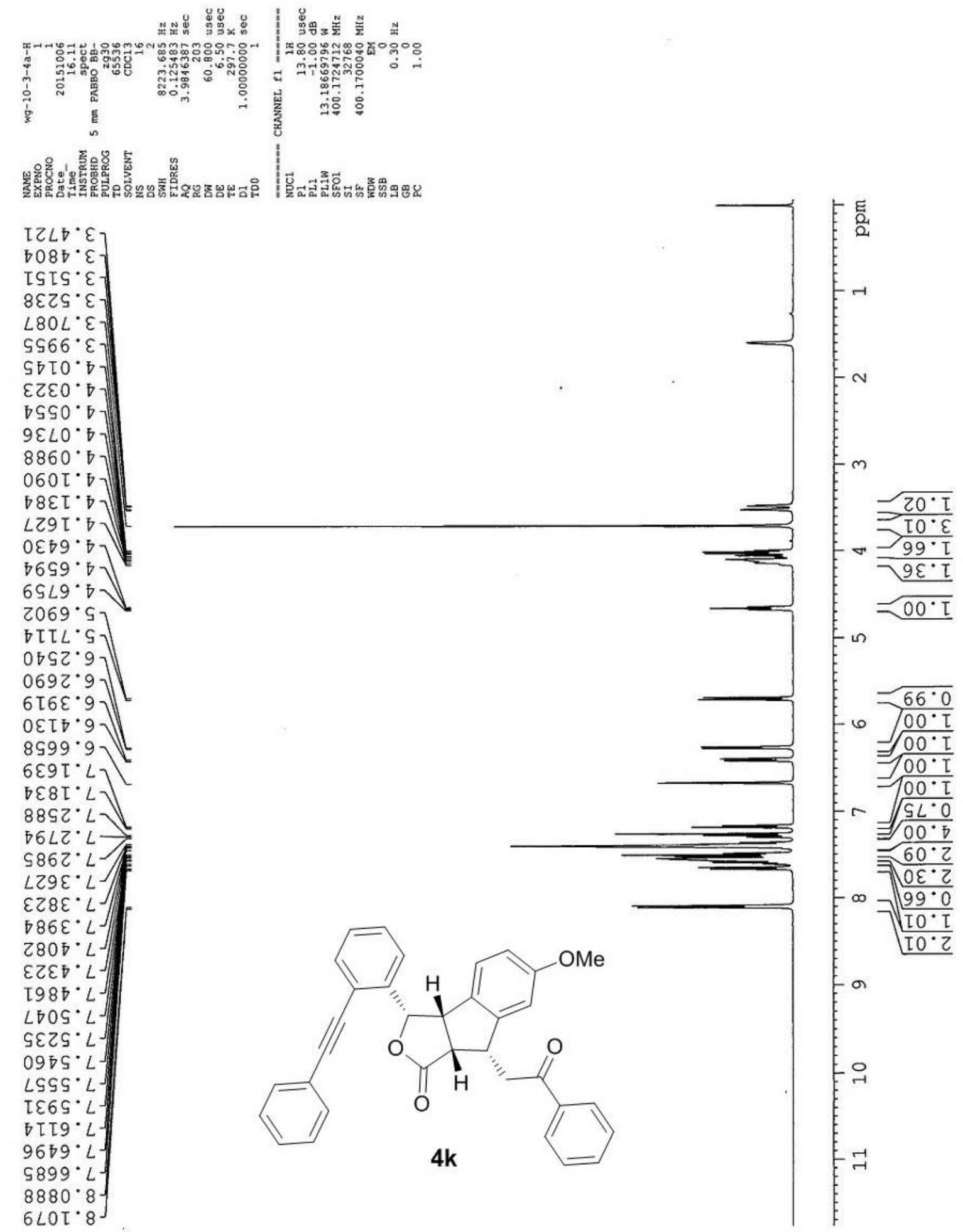




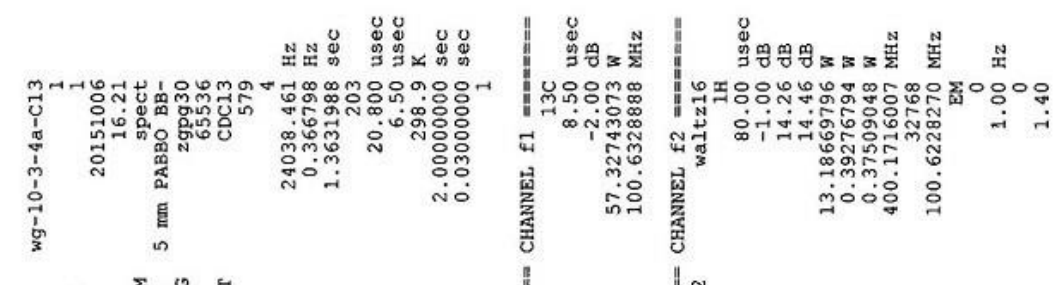

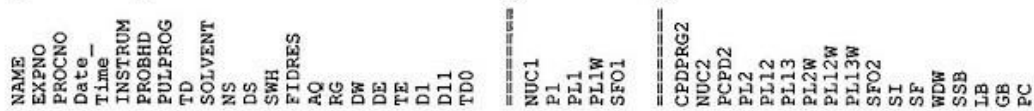

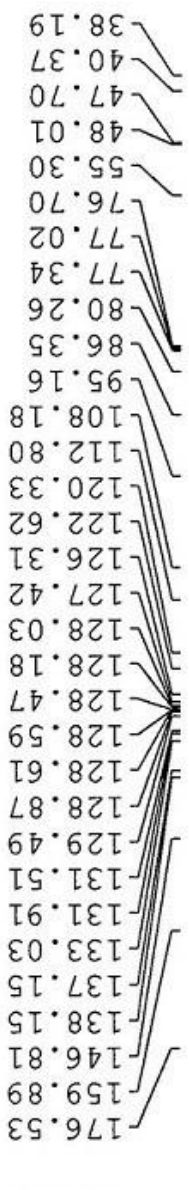

IE. $66 \tau-$

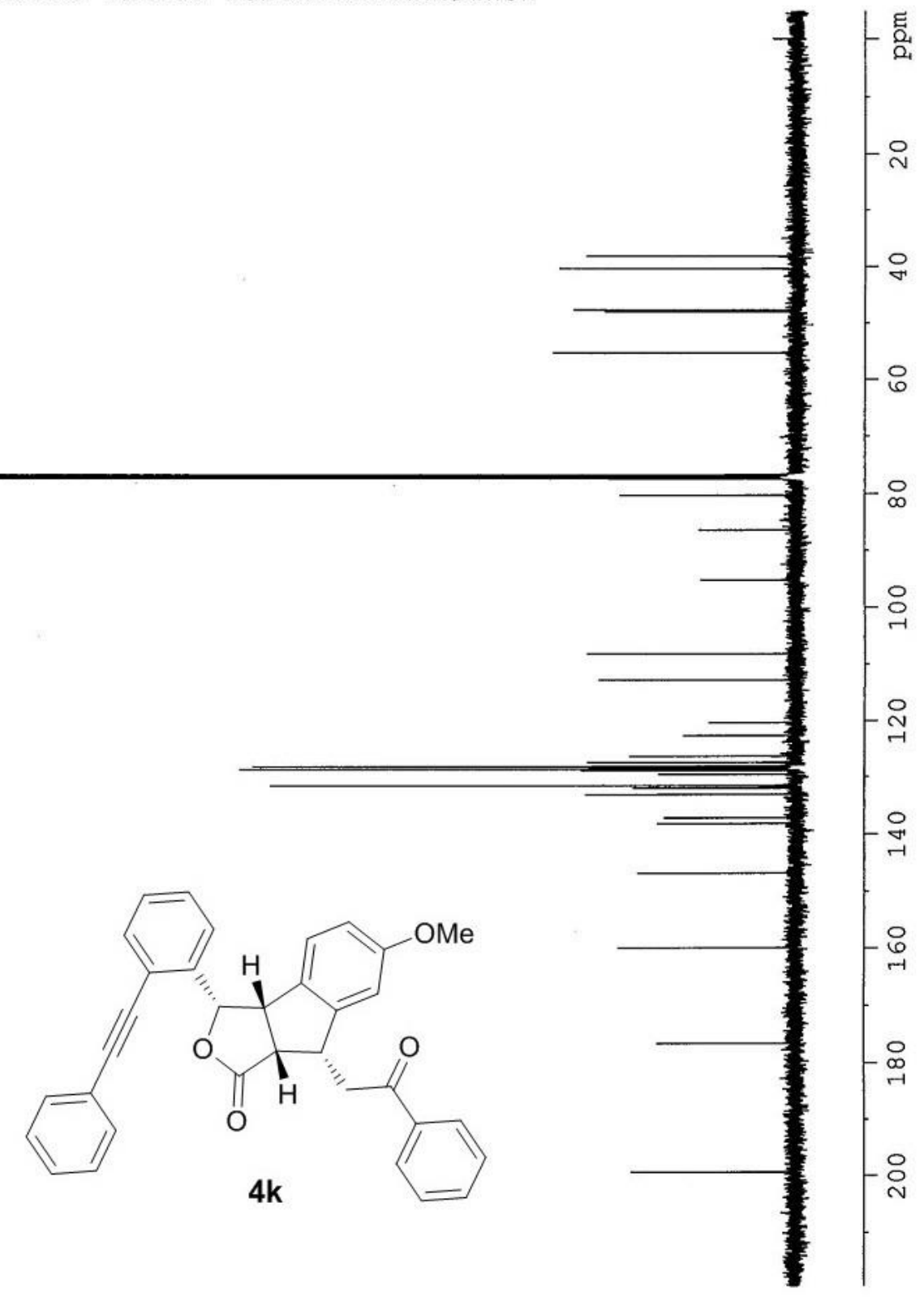




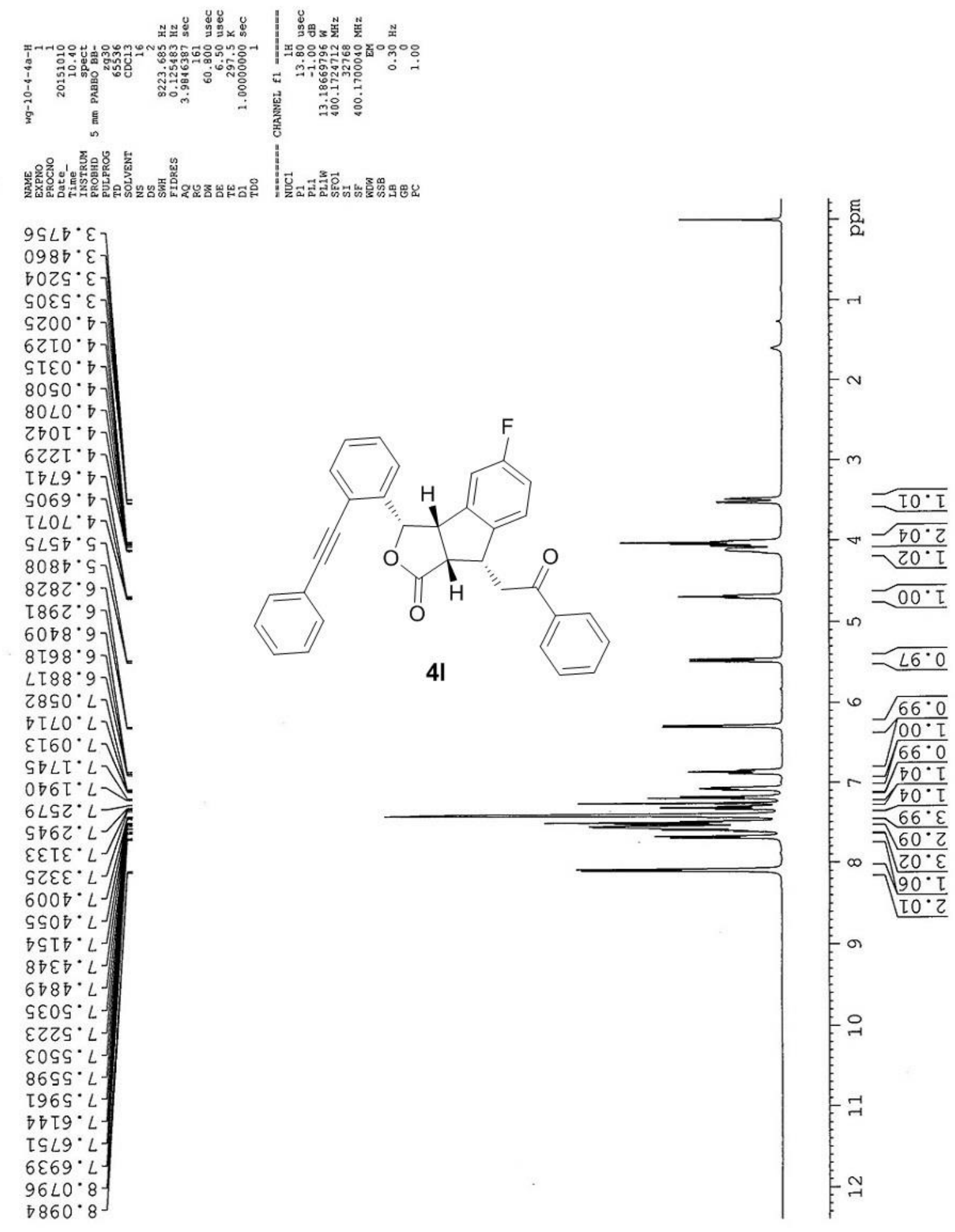



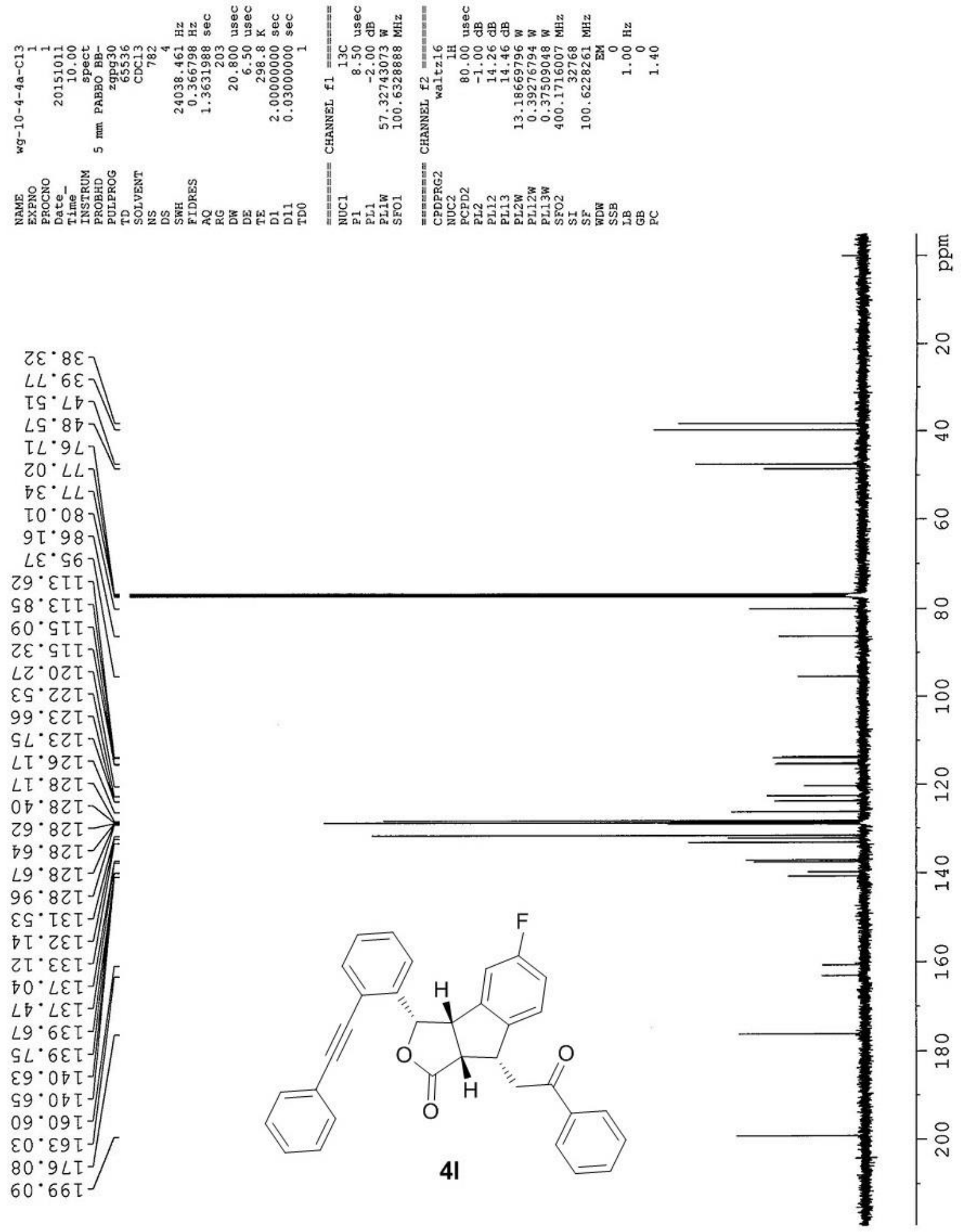

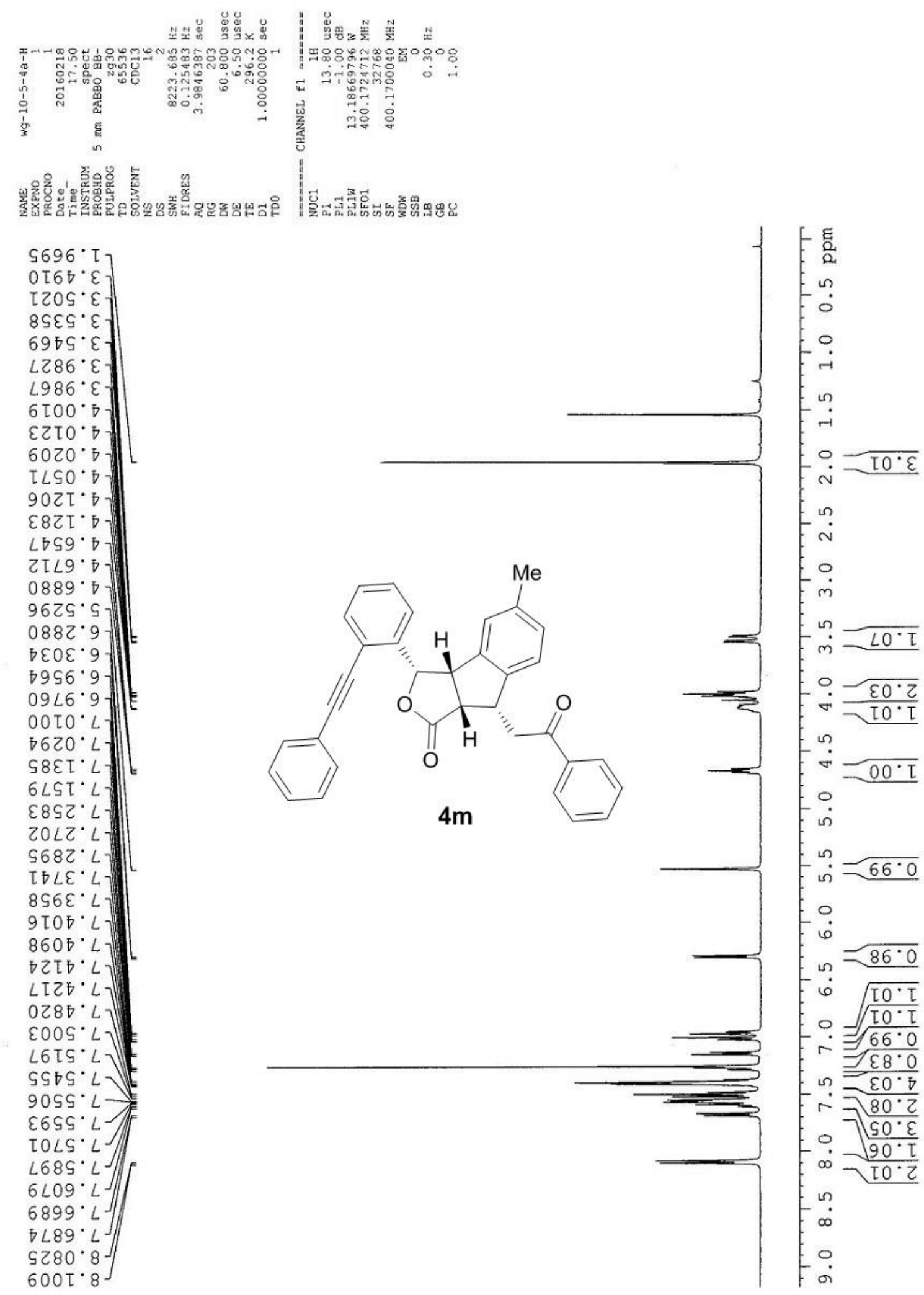


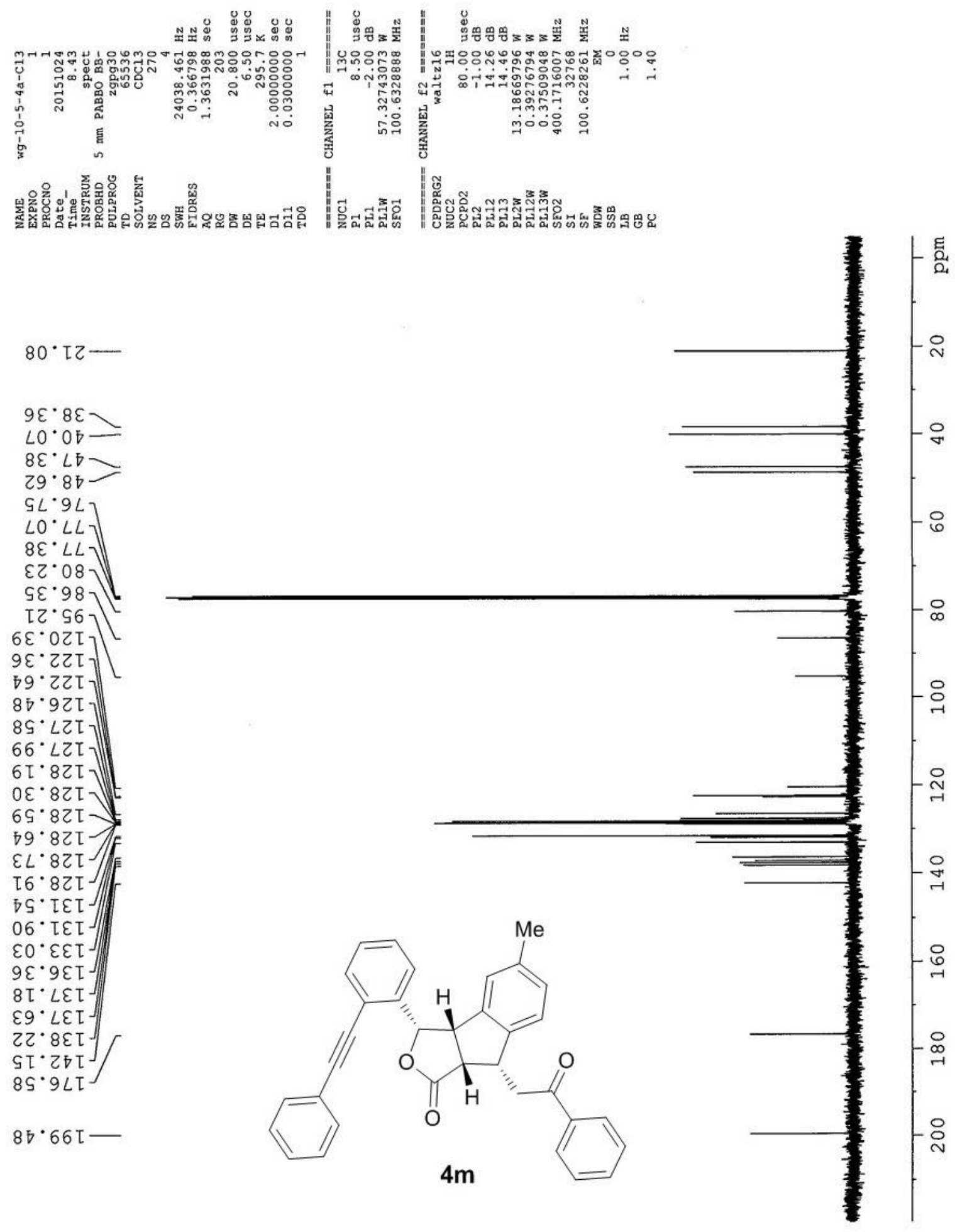



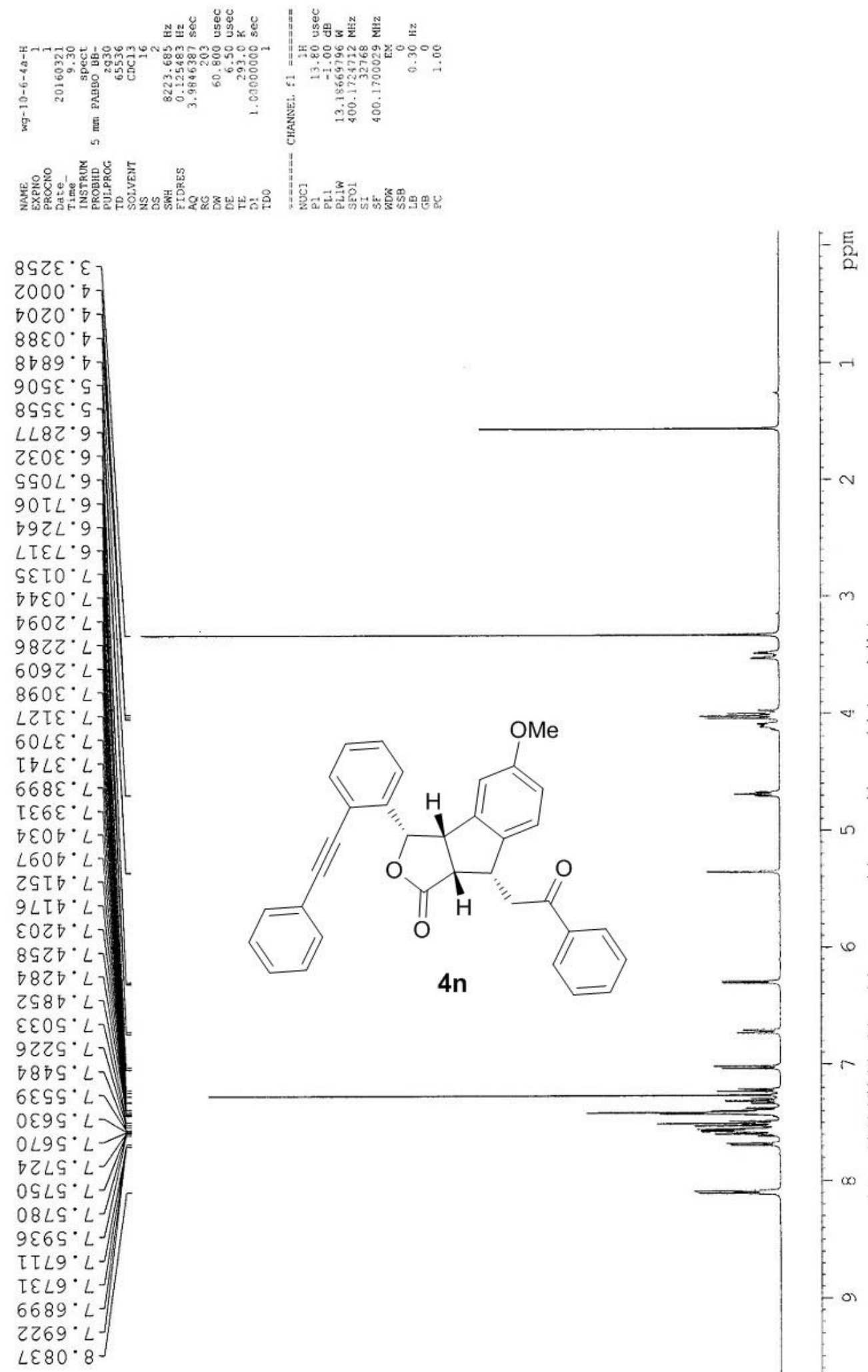

- 点

$\frac{L 0 \cdot \varepsilon}{S 0 \cdot I}$

$\frac{\frac{10}{80} \cdot}{2}$

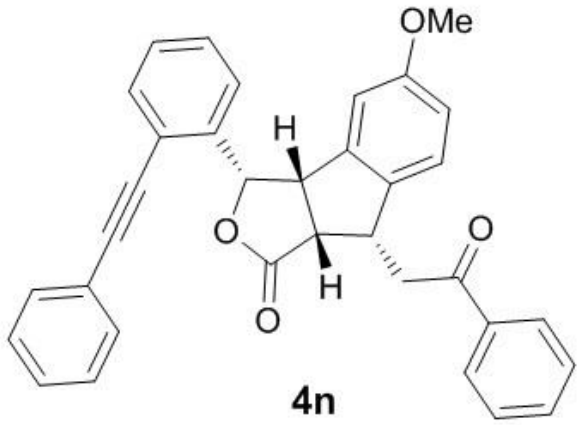

$=00^{\circ}$

-

$=00$.

1

$-66.0$

$\sqrt{66 \cdot 0}$

150

r

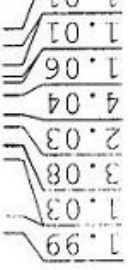

- 


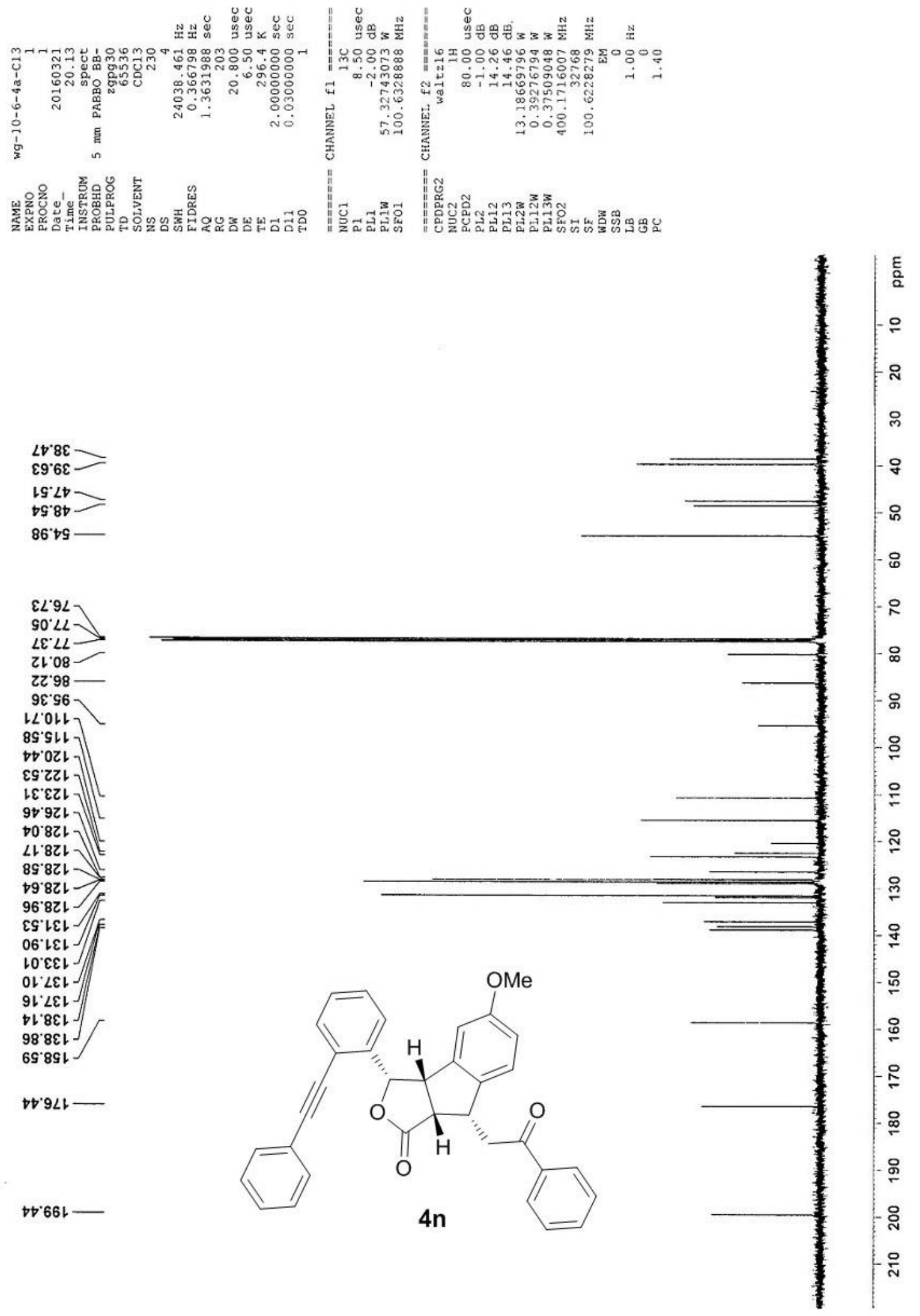




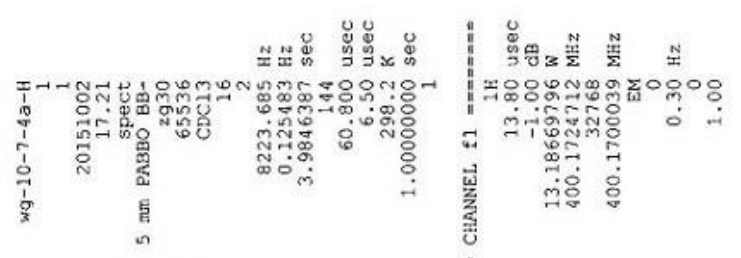

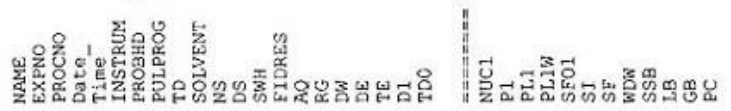
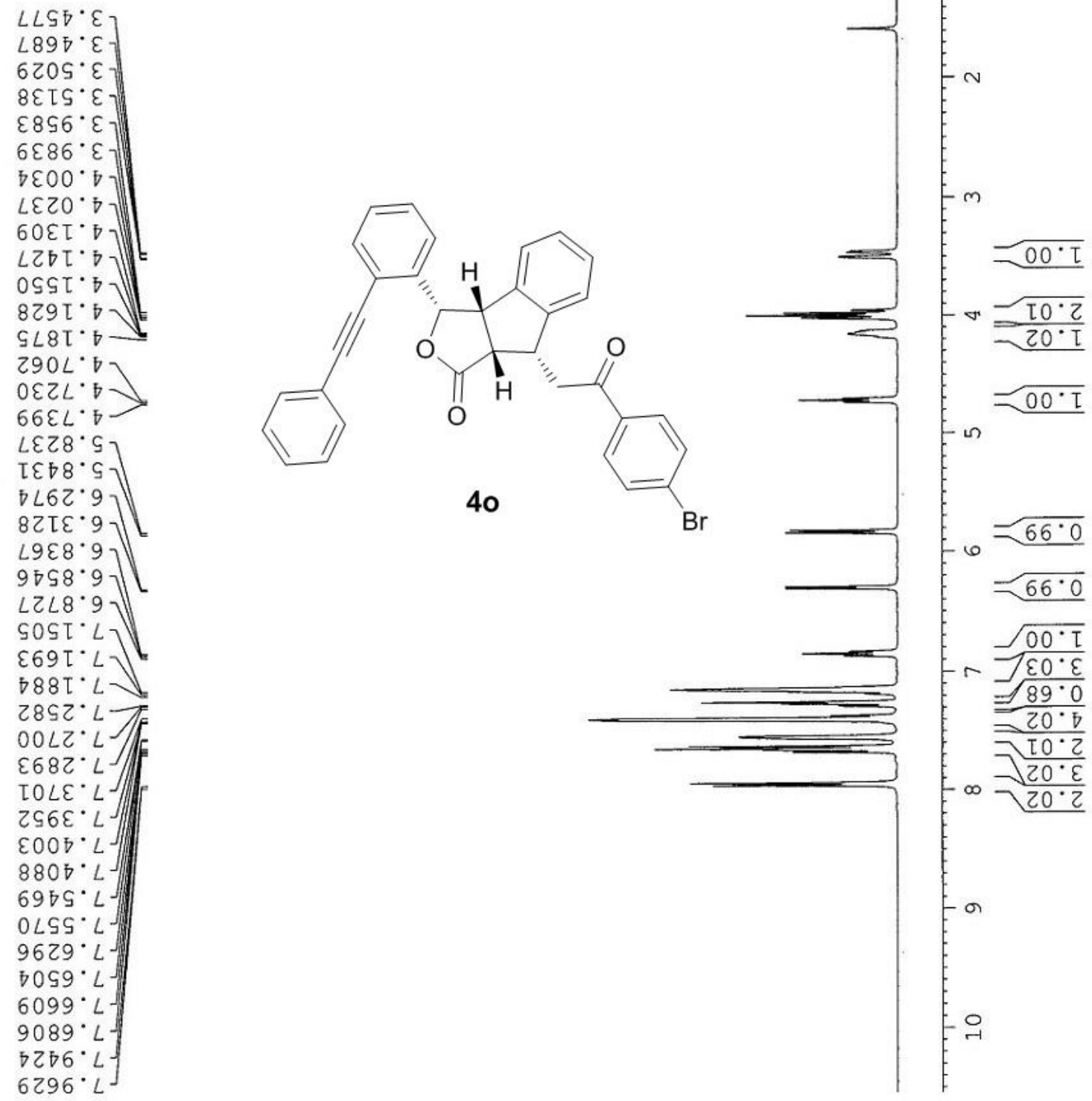


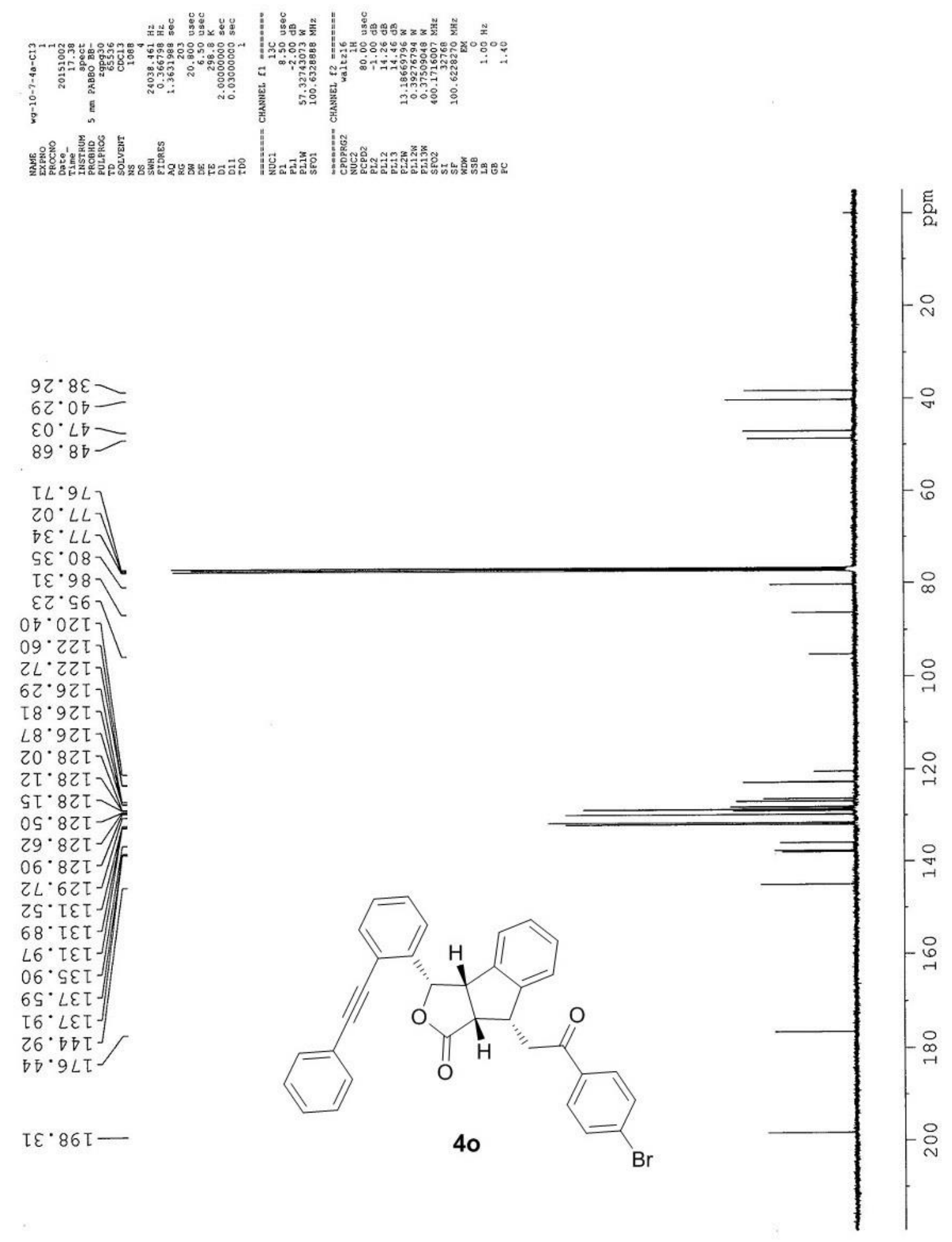



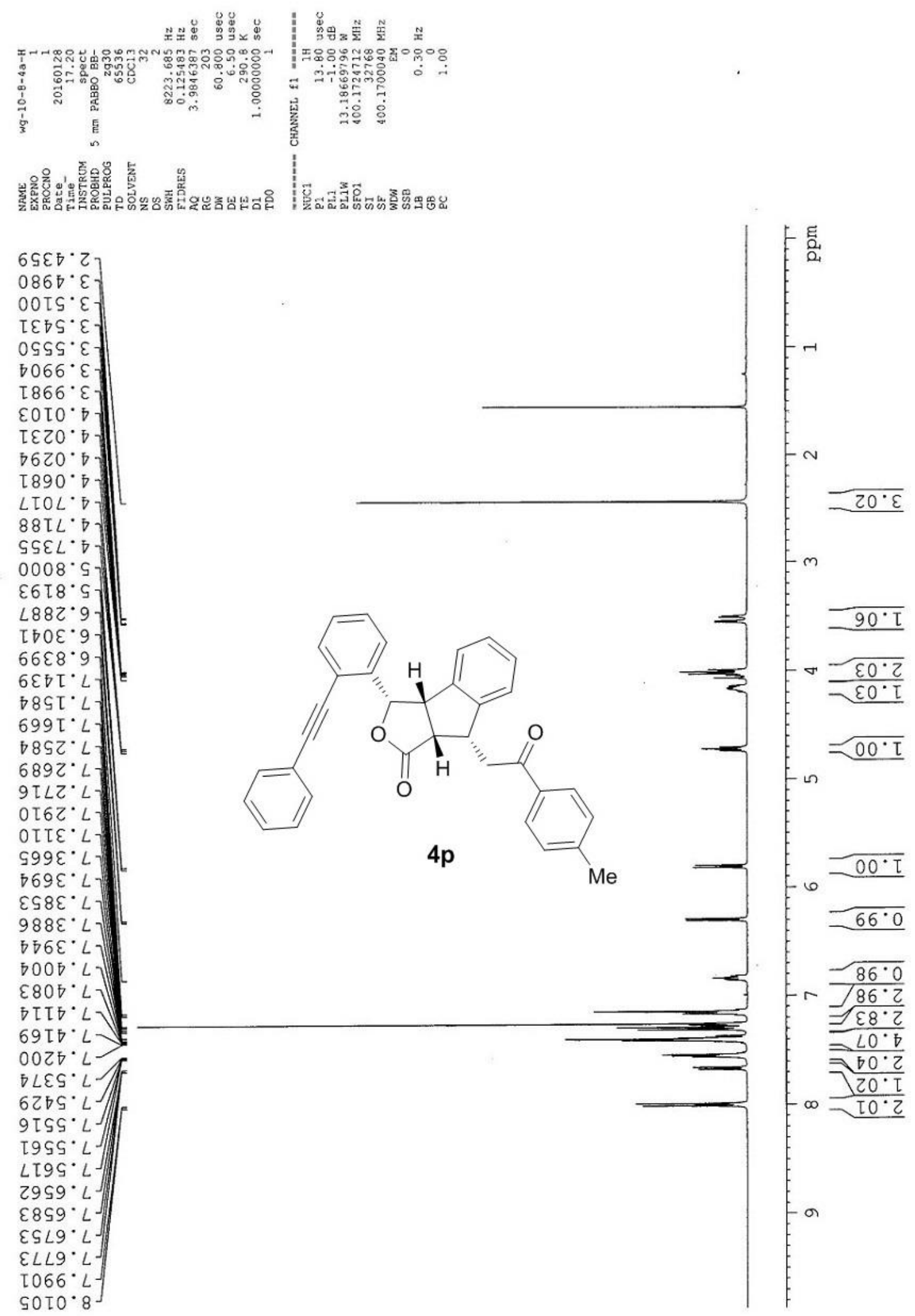


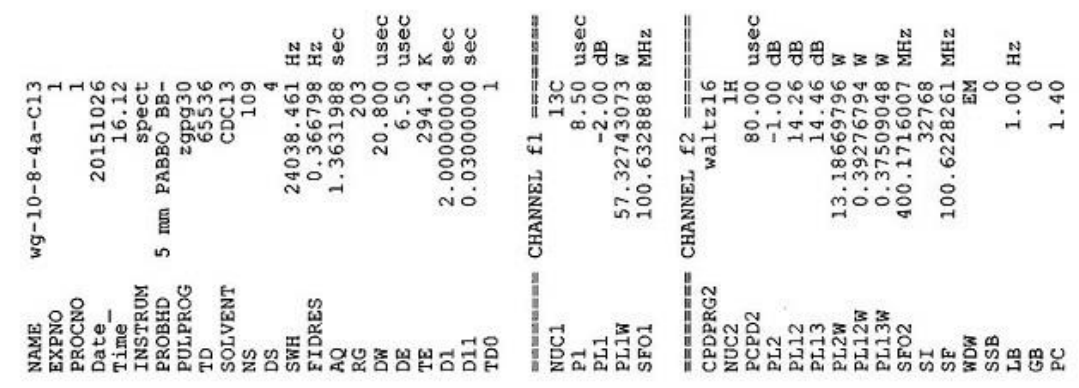

$Z L I Z$

$600^{\circ} 8 \varepsilon$

$8 \varepsilon^{\circ} 0 \mathrm{t}-$

$\angle Z \cdot L b=$

8L' $9 L$

OL'LL

$2 t \div 2 L$

$9 z 08$

$8 \varepsilon^{\prime} 98-$
GZ' $96-$

$8 \varepsilon .0 Z 1$

Z9'ZZL -

s8'ZZL

$\varepsilon \varepsilon ' 9 z \downarrow$

9L'9ZL

00 '8zL

เᄂ.8Zᄂ

$\varepsilon \varepsilon^{\prime} 8 z \downarrow$

$99 \cdot 821$

86.82\}

เE.6ZL 5

ts'lहl f

G9'เع!

$09^{\circ} \angle E$ L

$\angle 0^{\circ} 8 \varepsilon 1$

$z 8$ ' $E$ -

เE'stl

$9 t^{\circ} 921$

$96.861-$

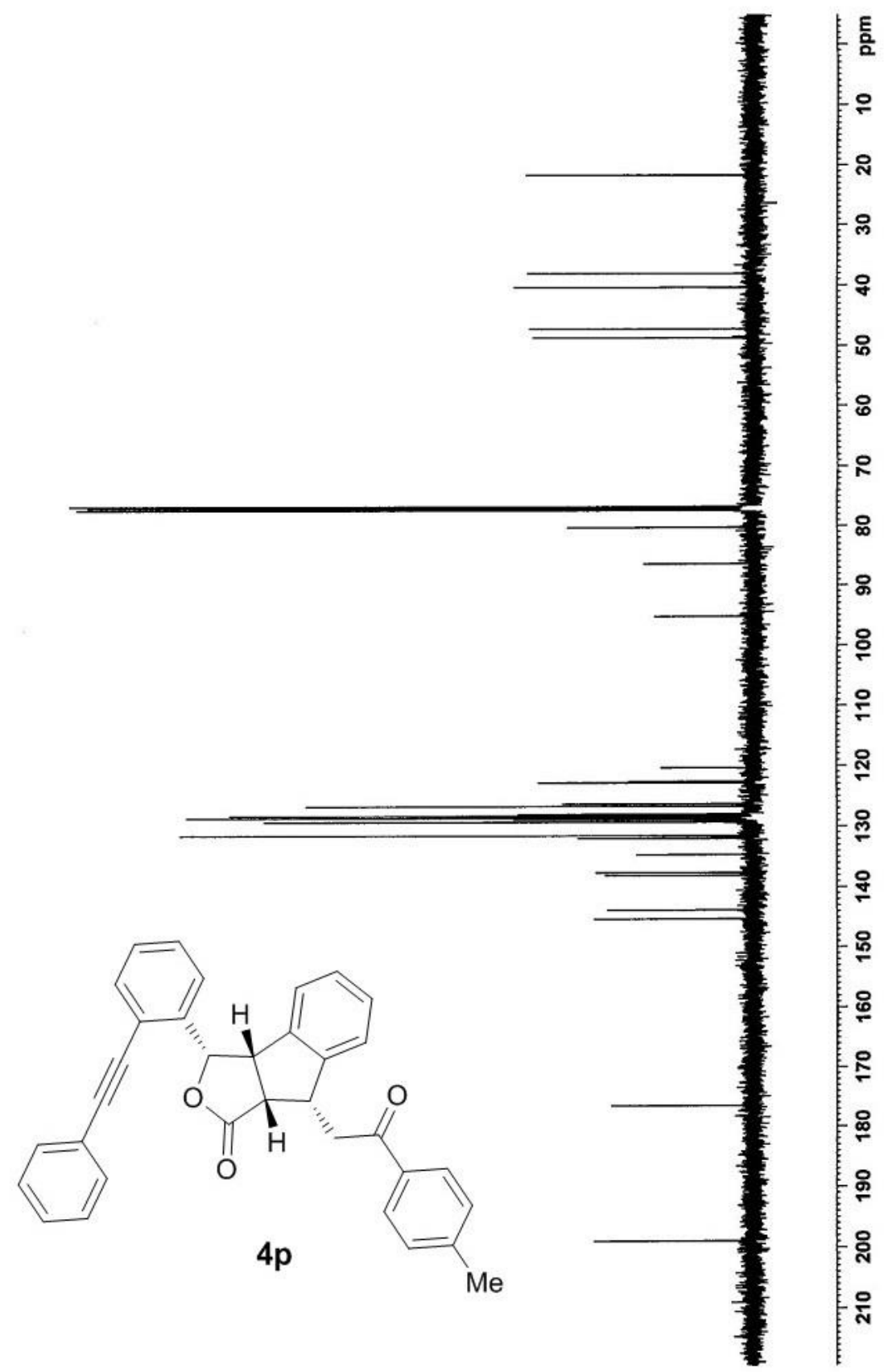



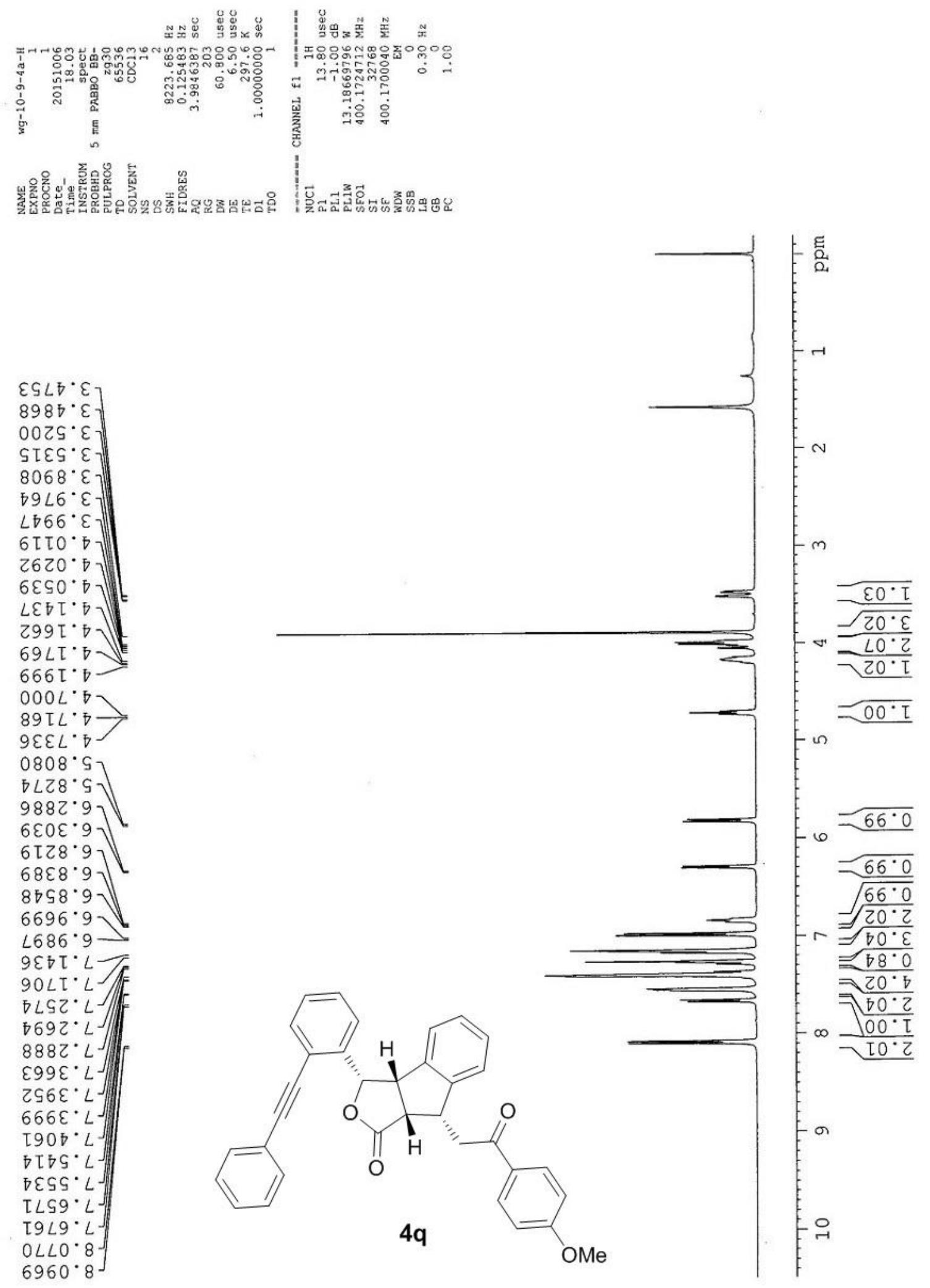


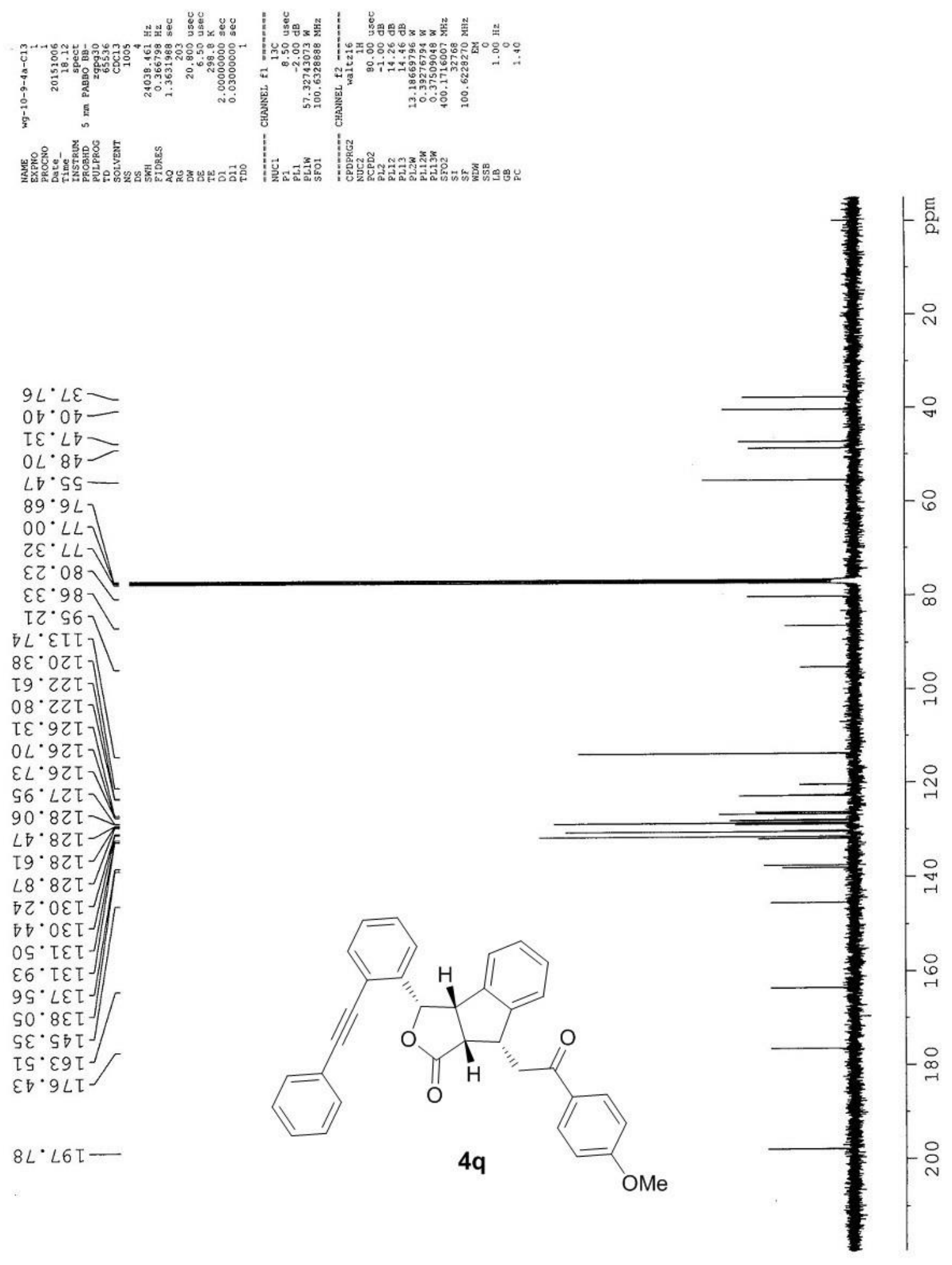




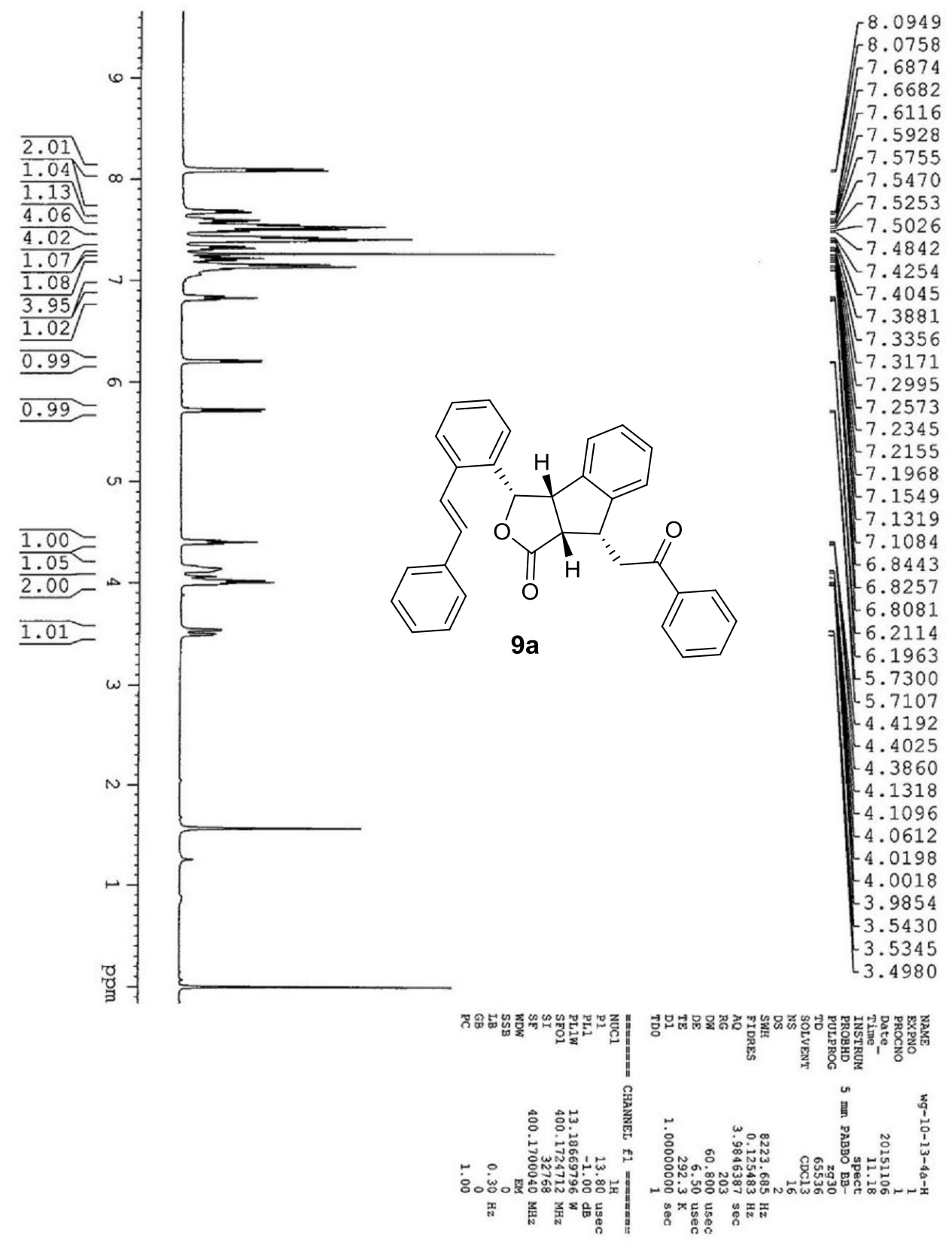




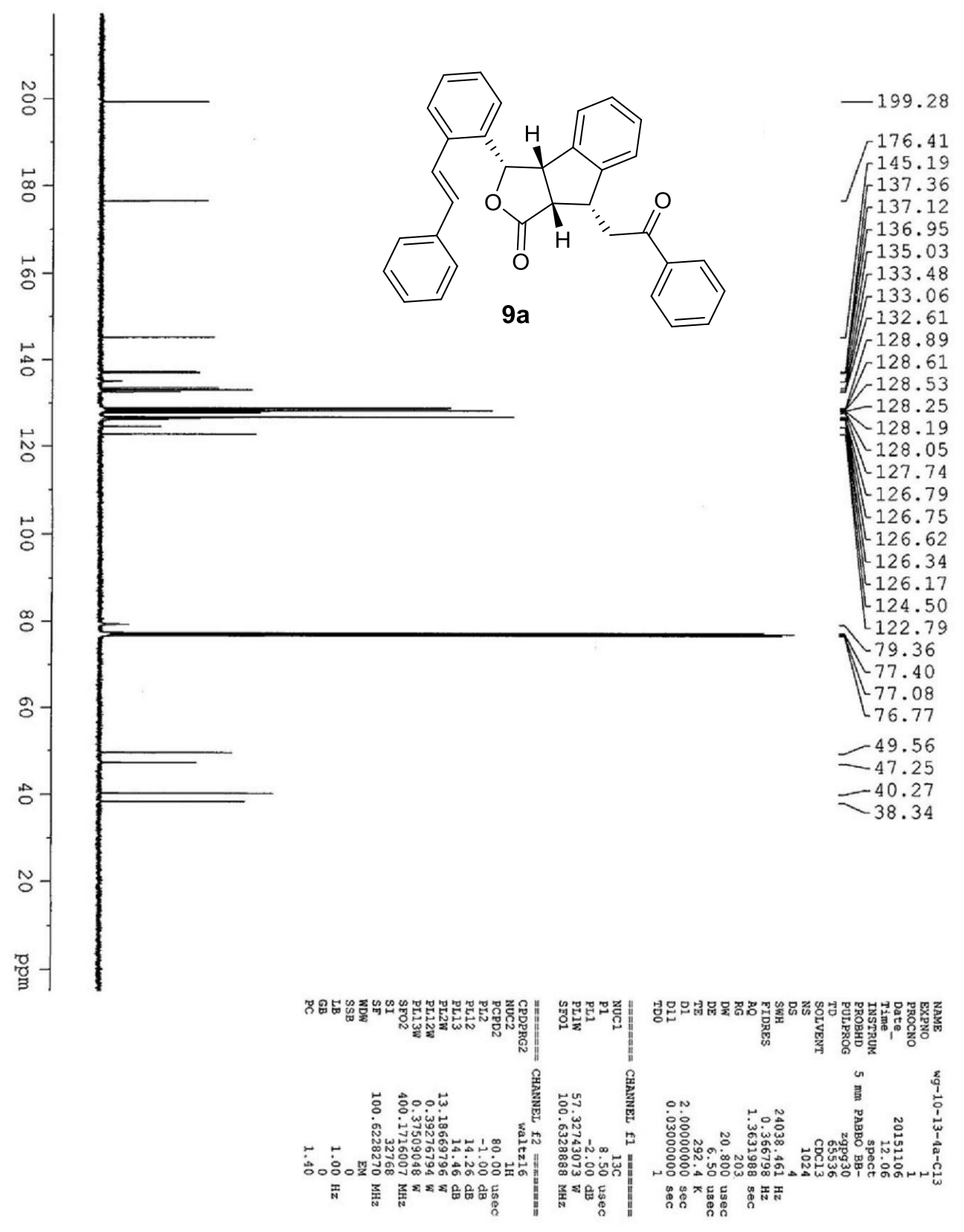




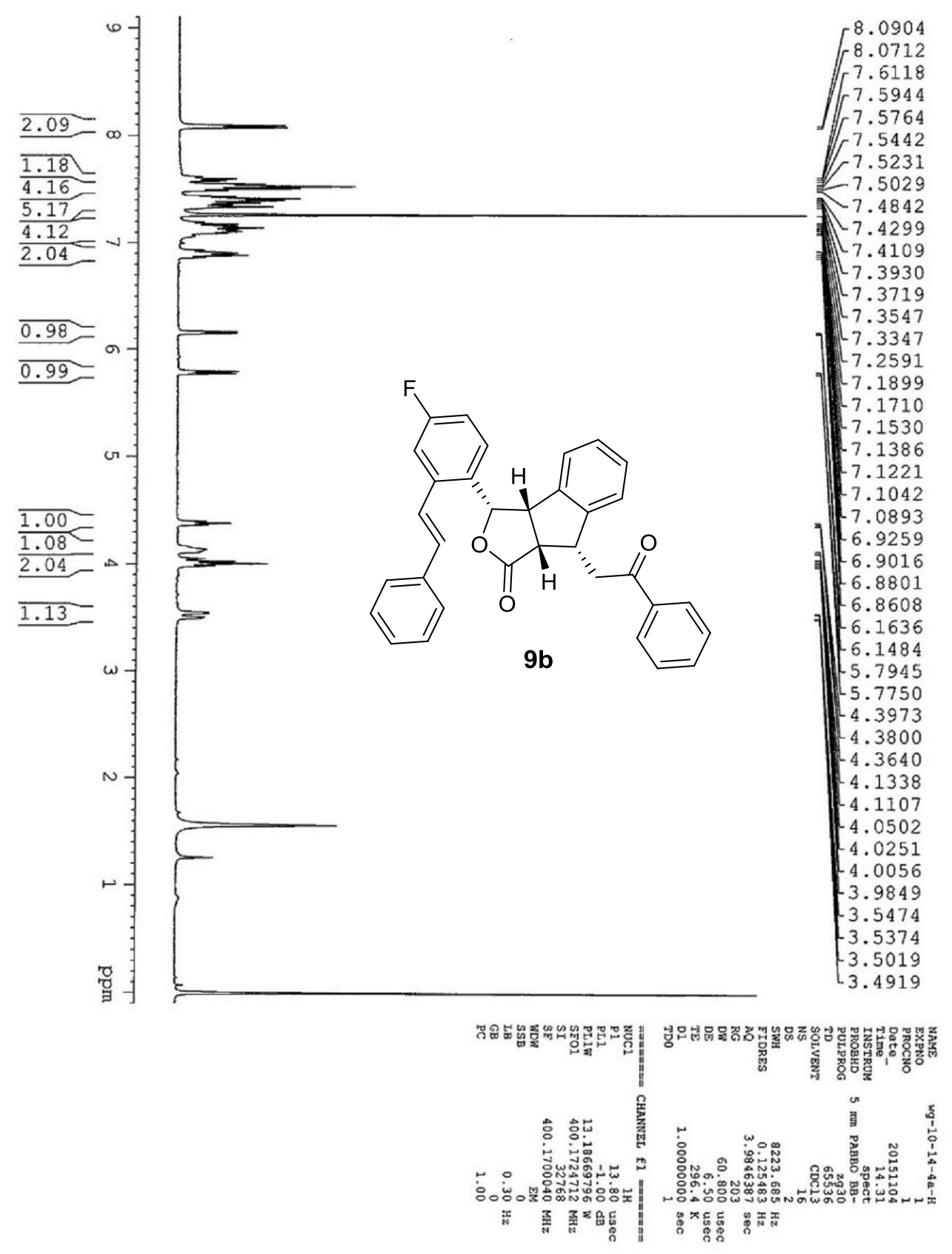




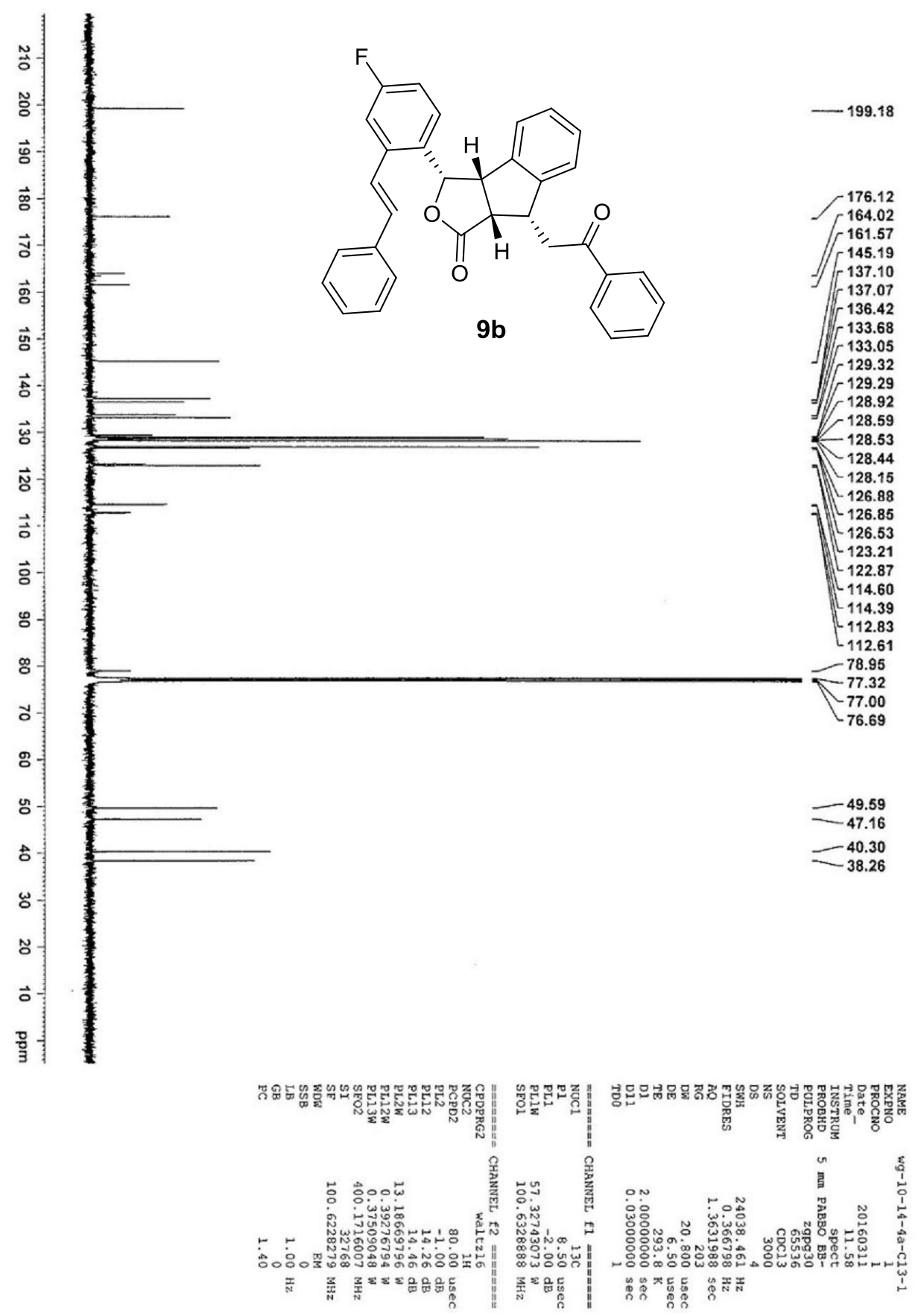




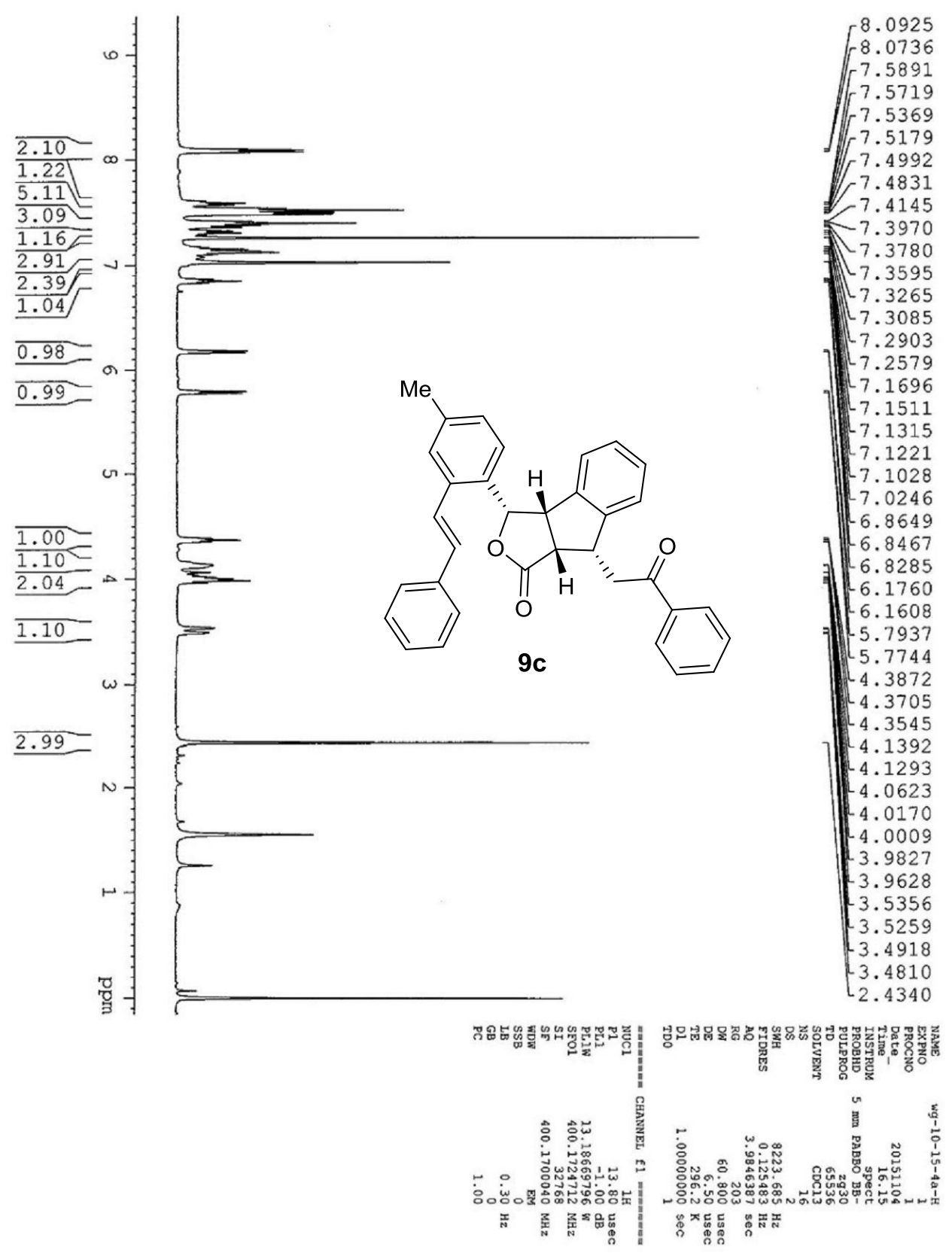




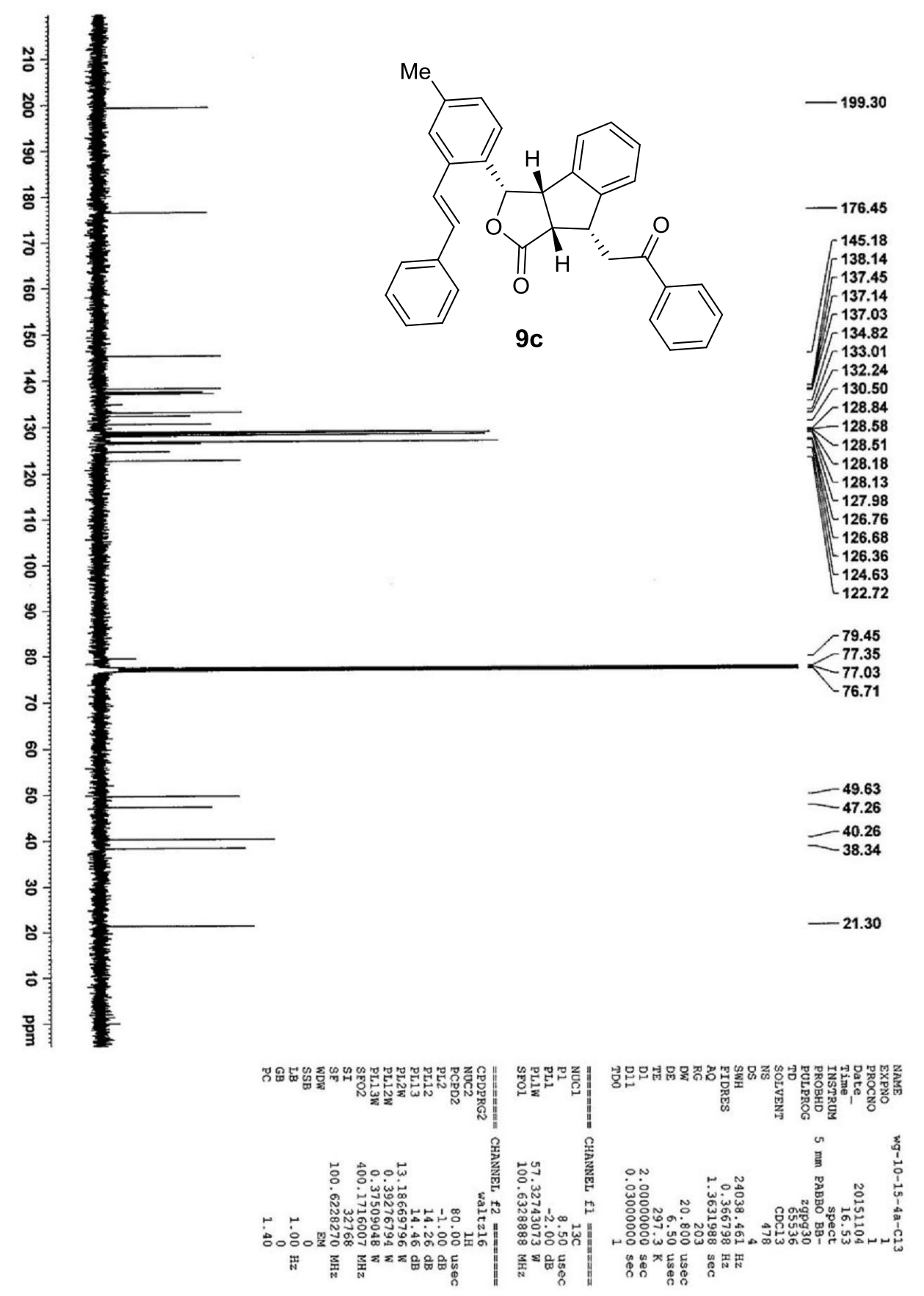




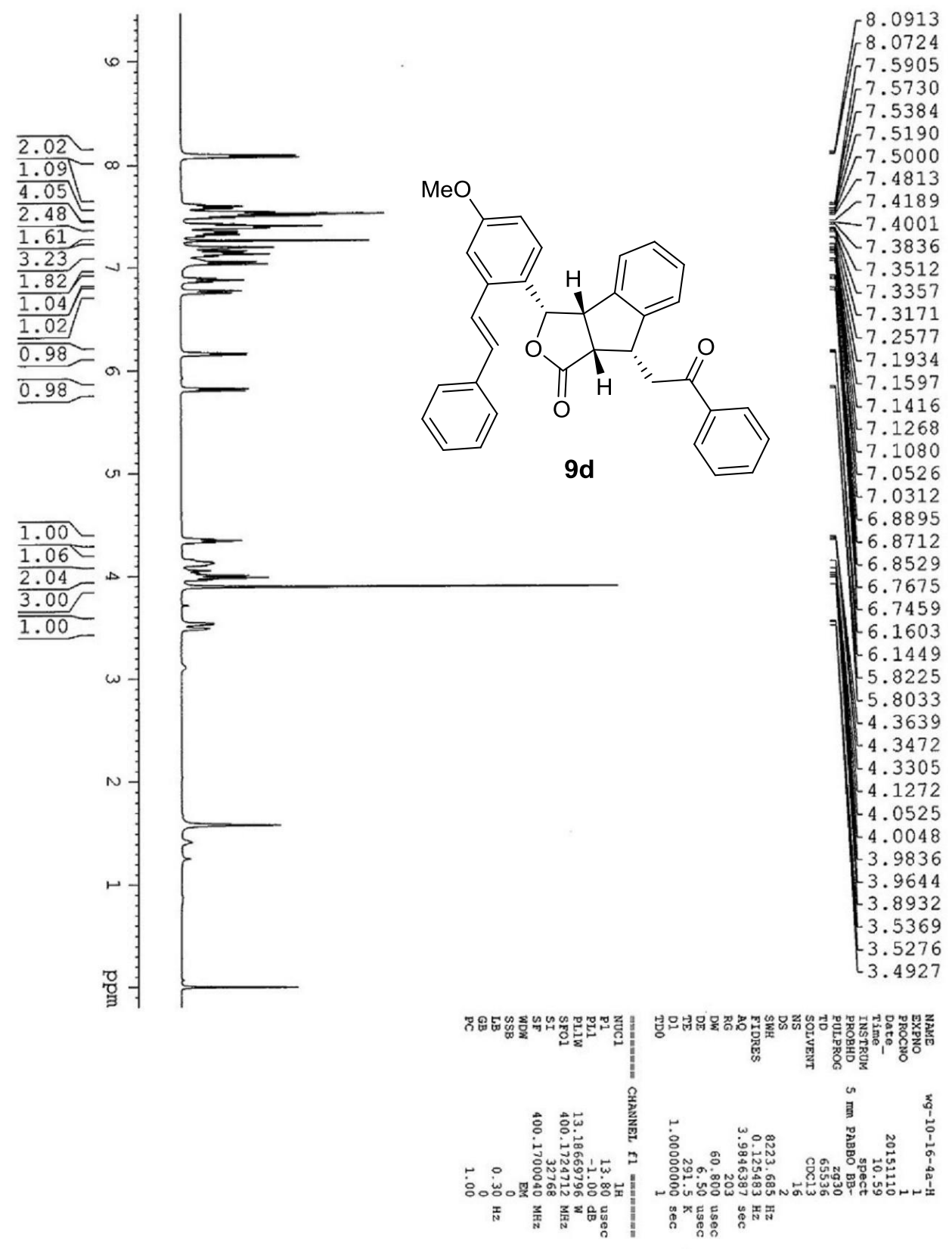



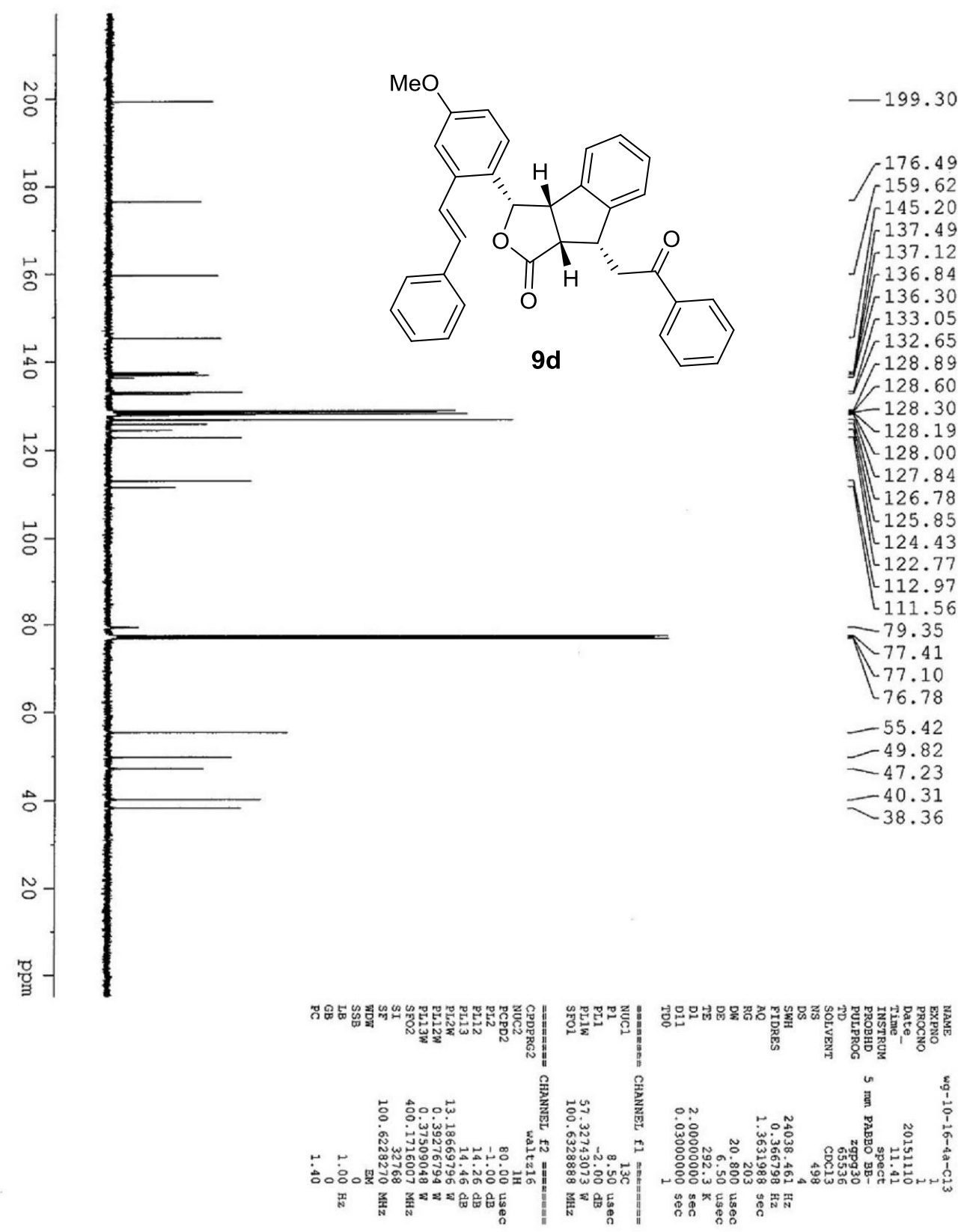


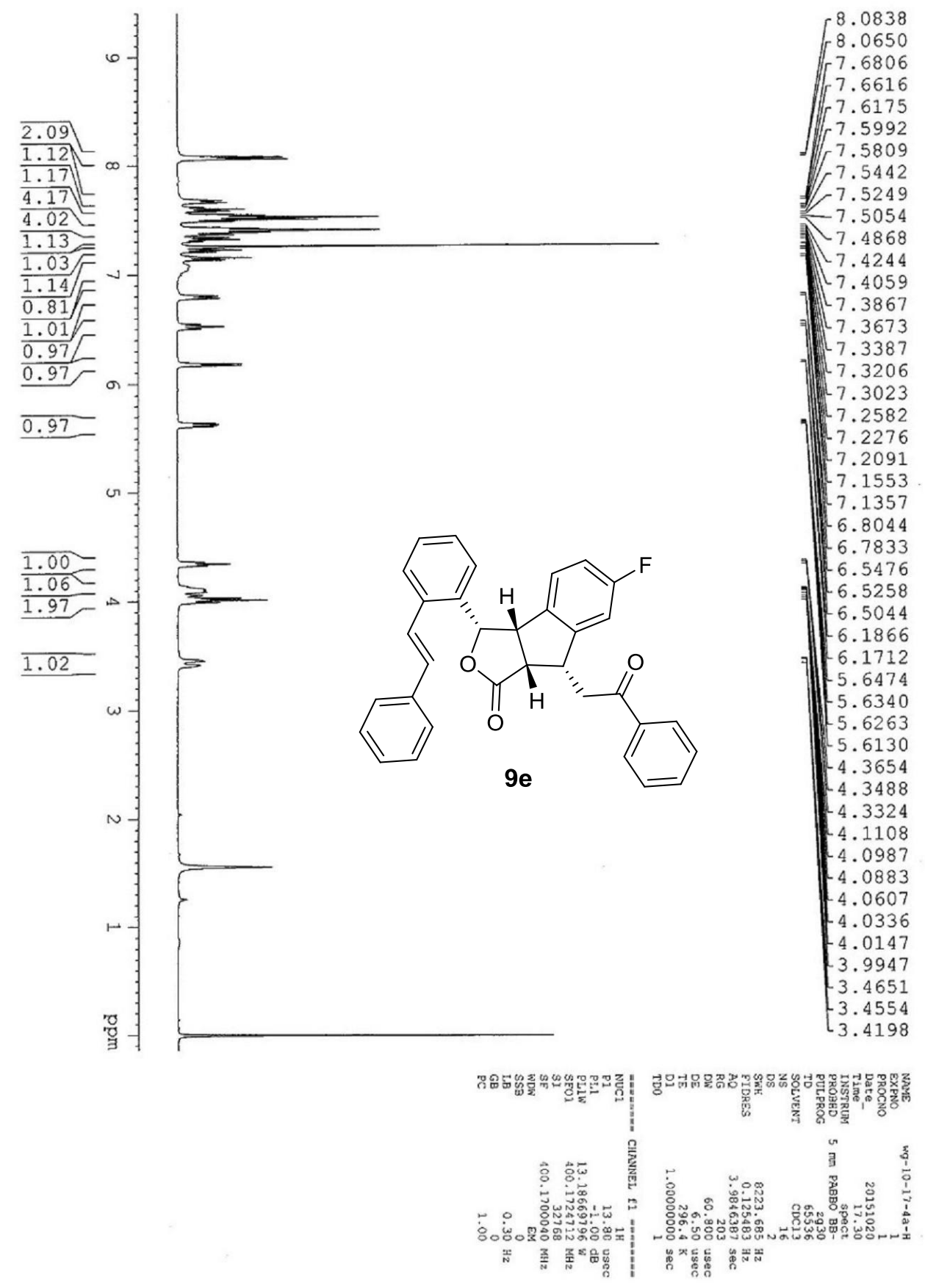




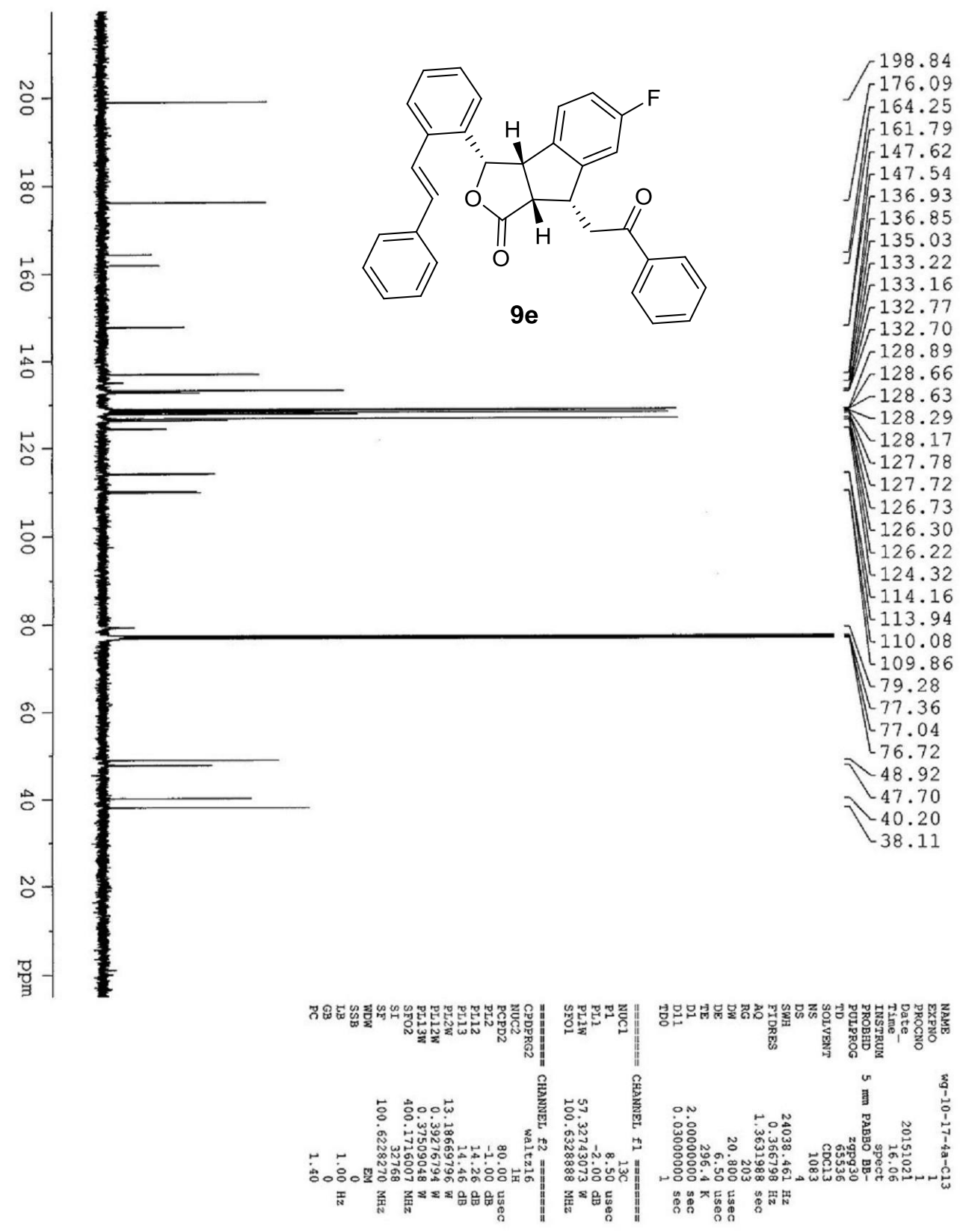




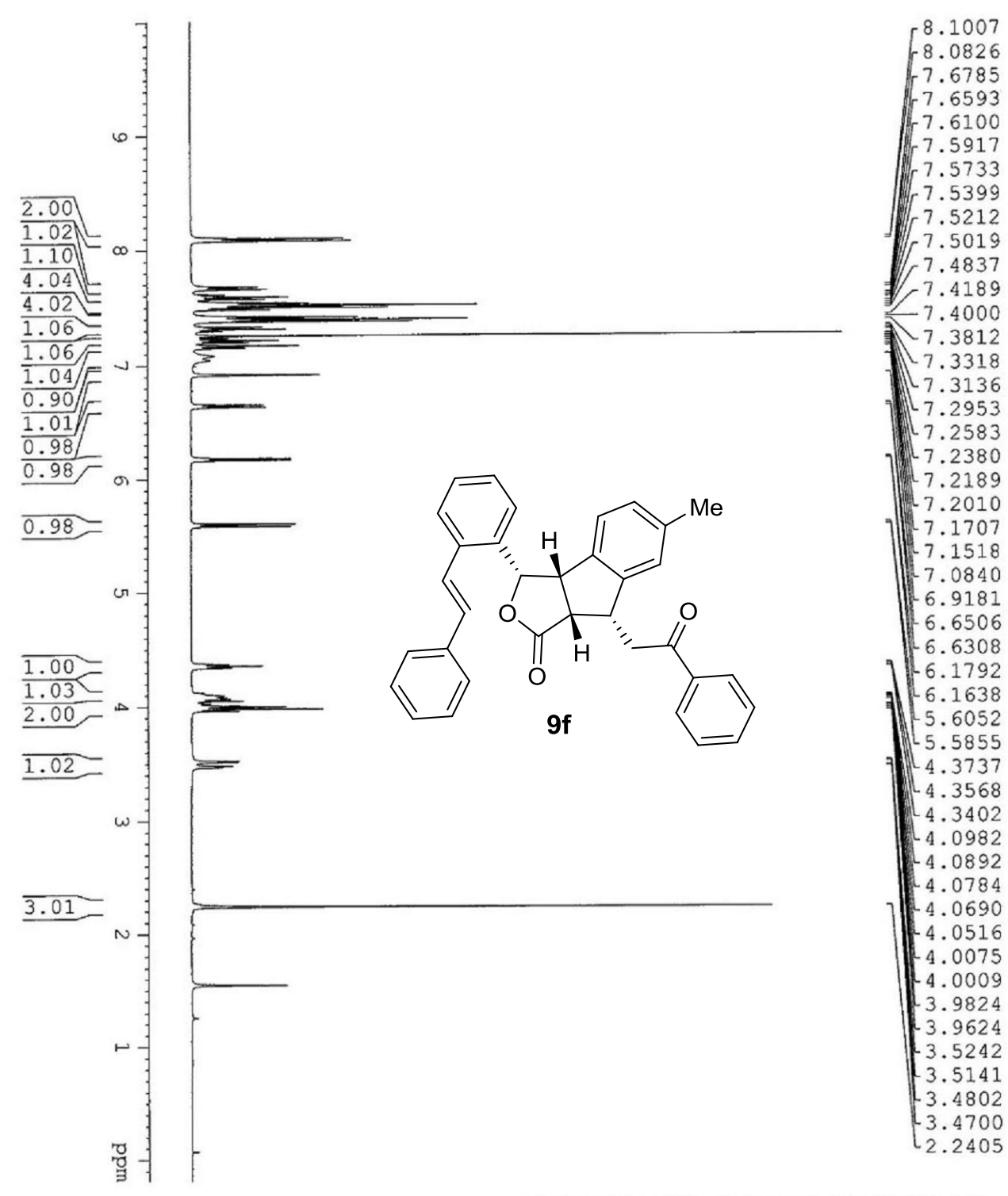

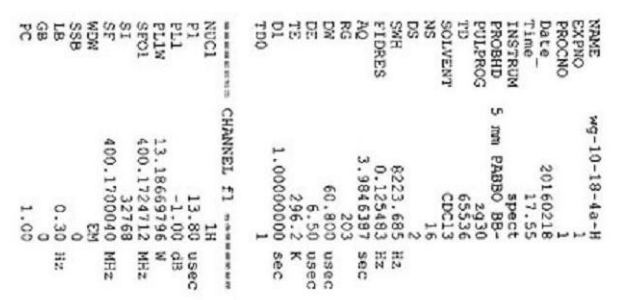




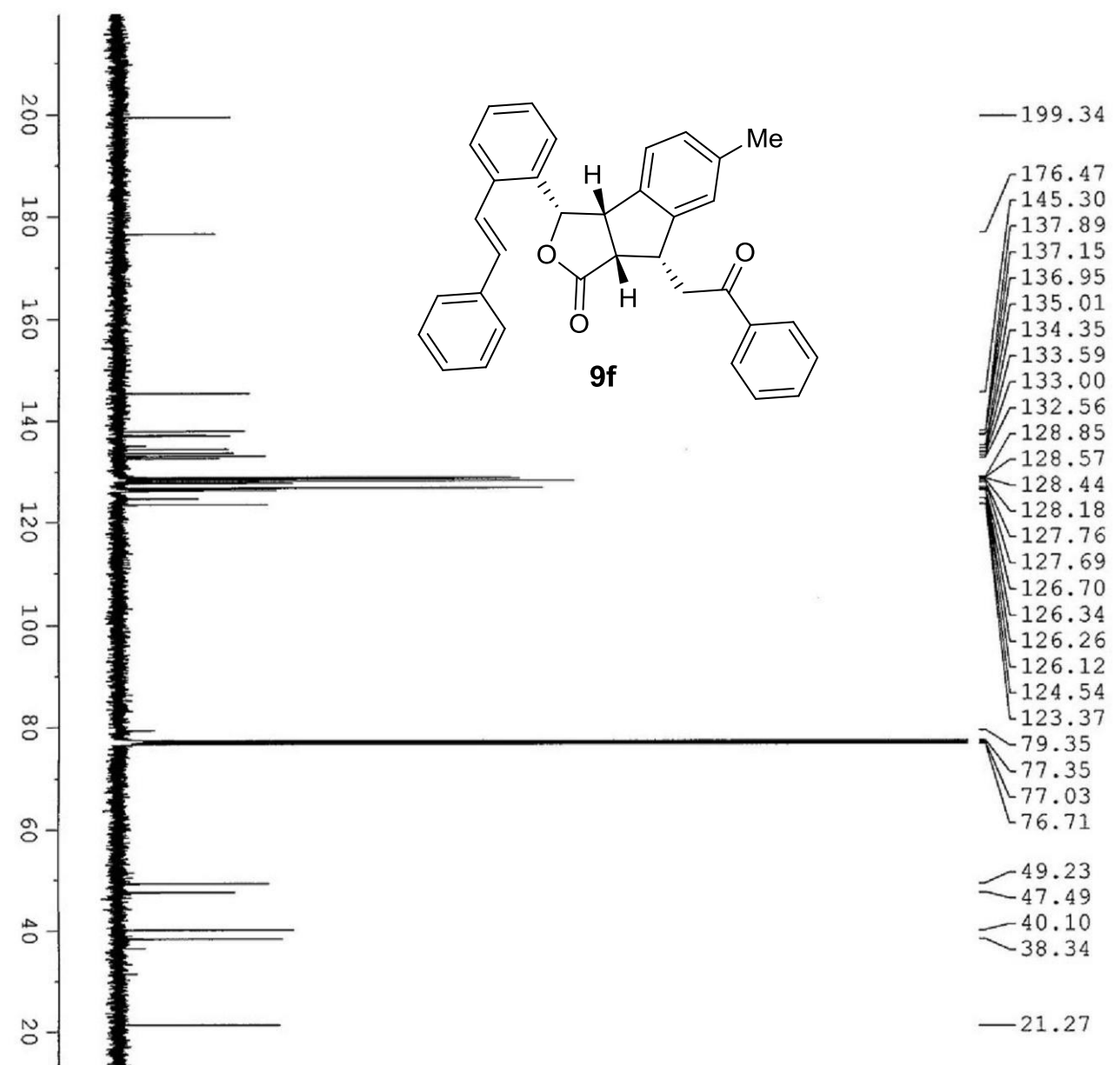

旁

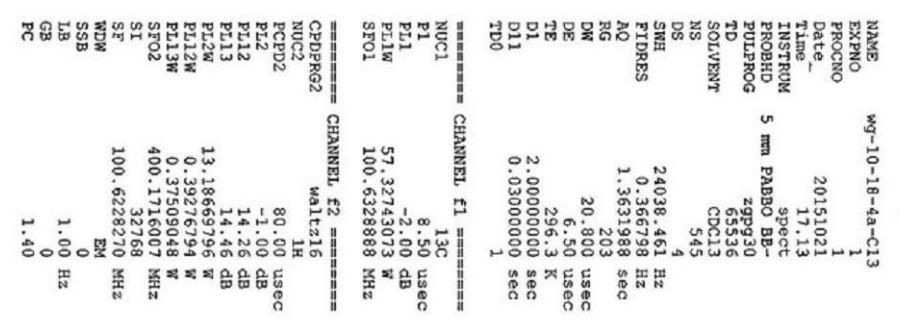




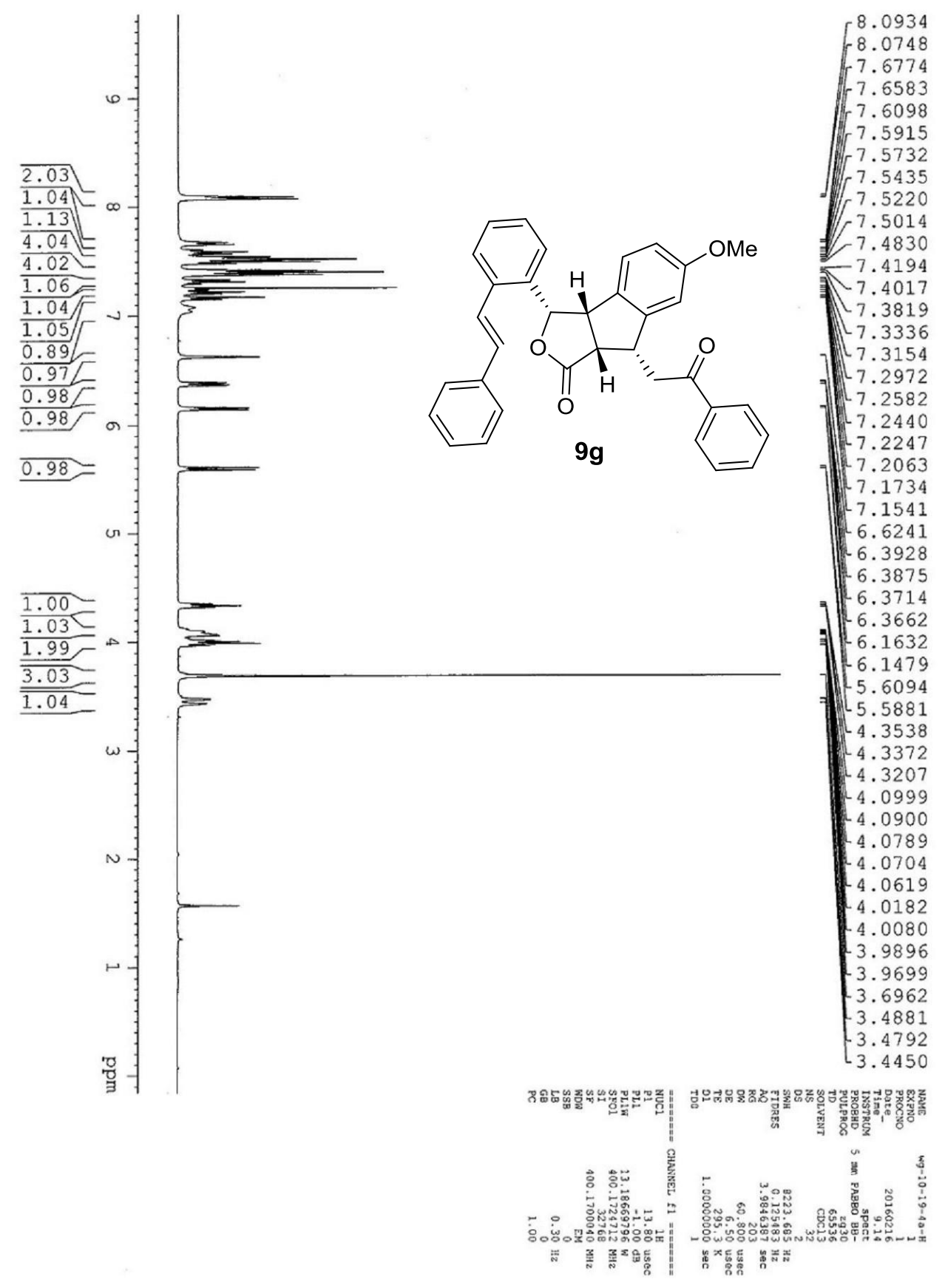




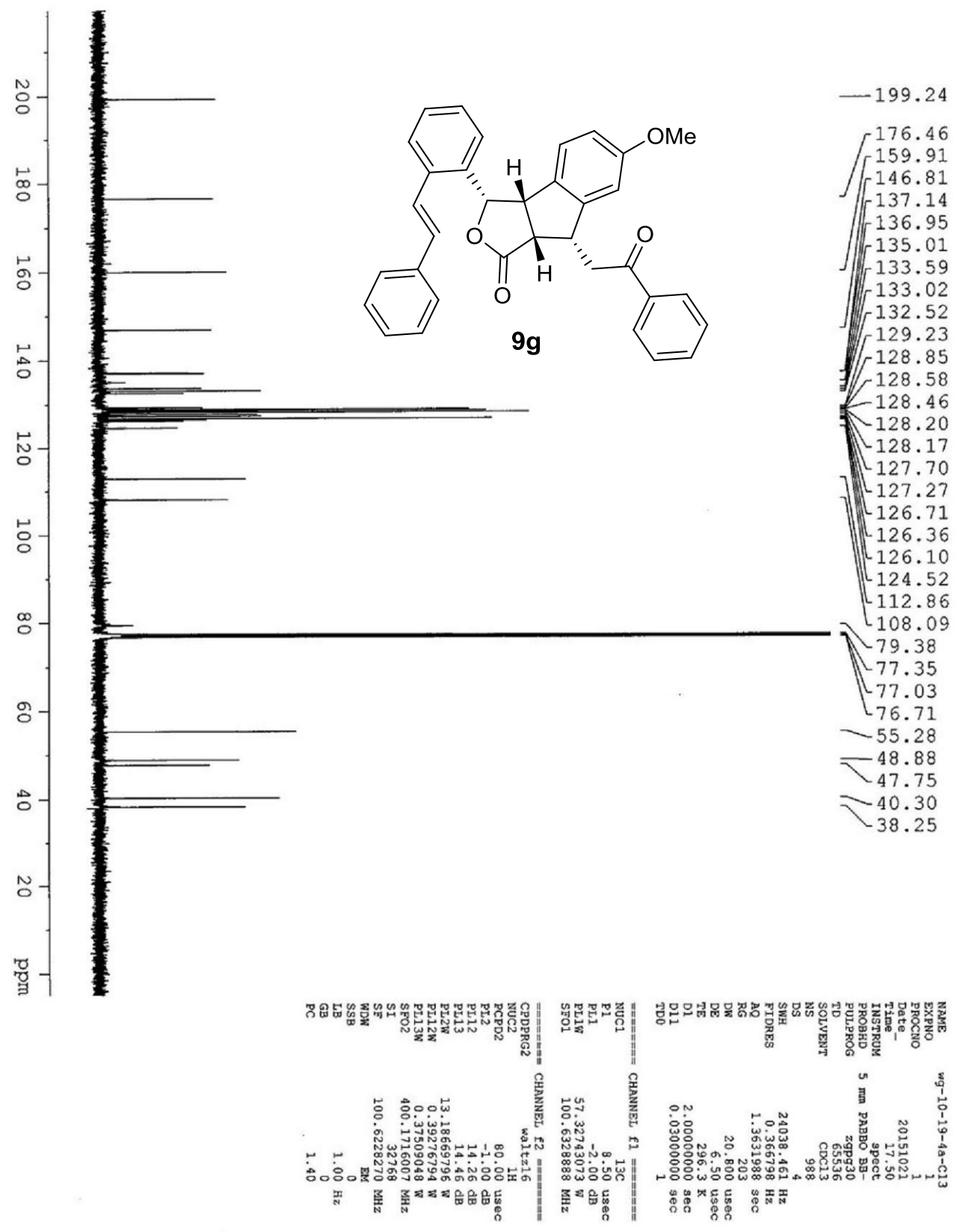




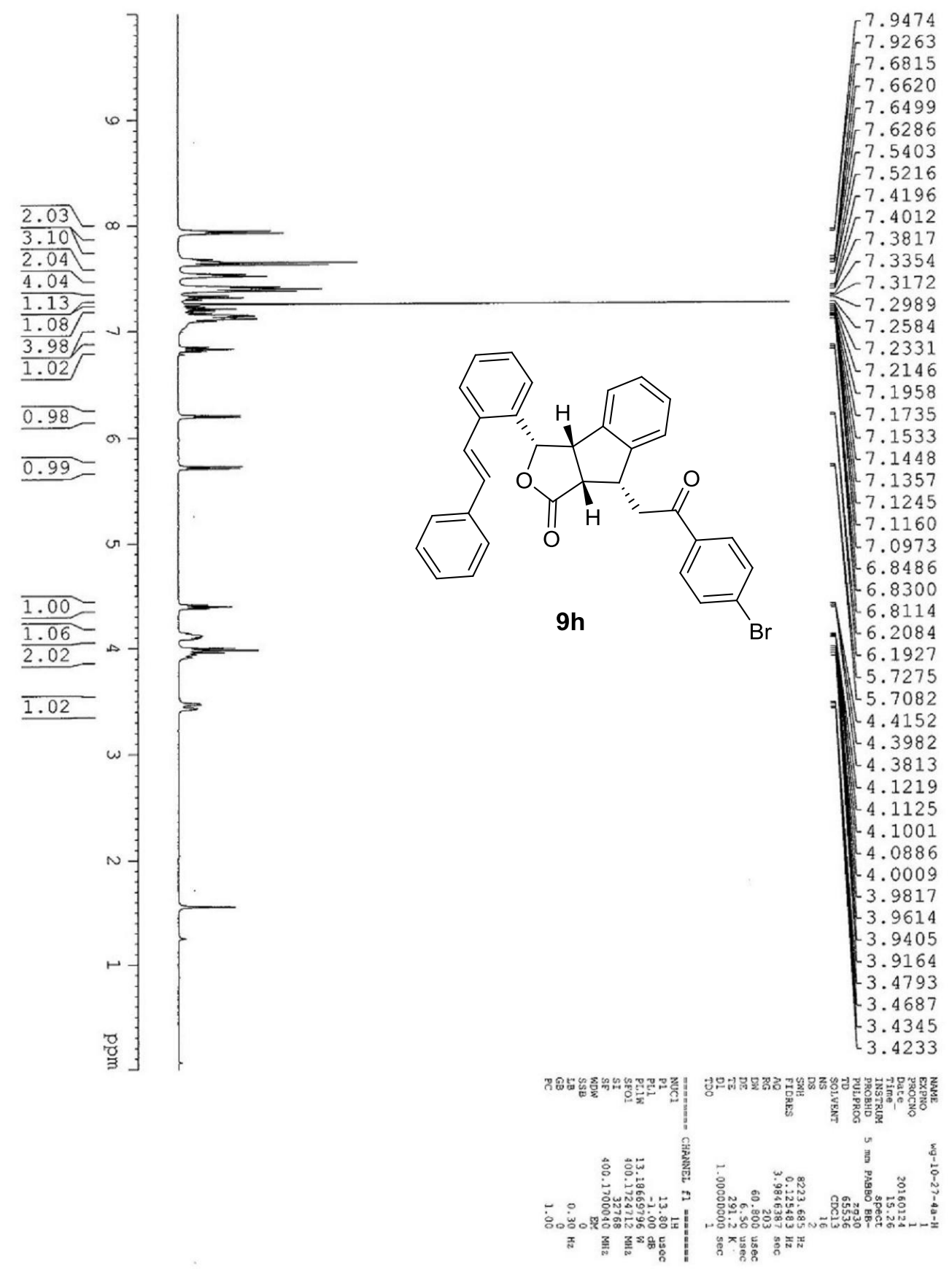




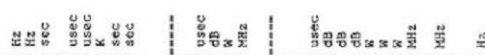

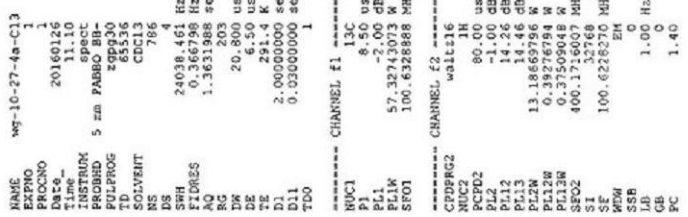

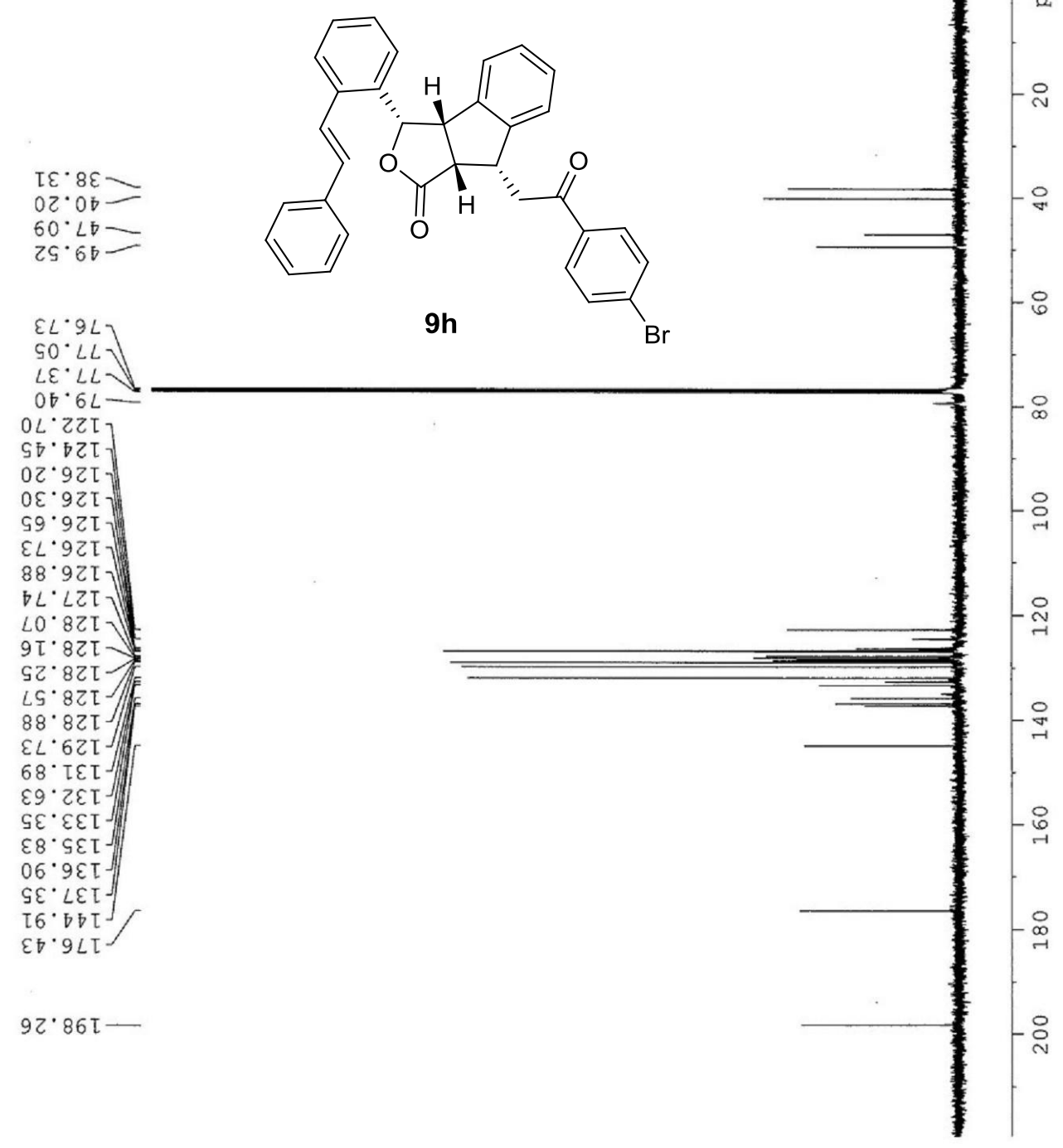




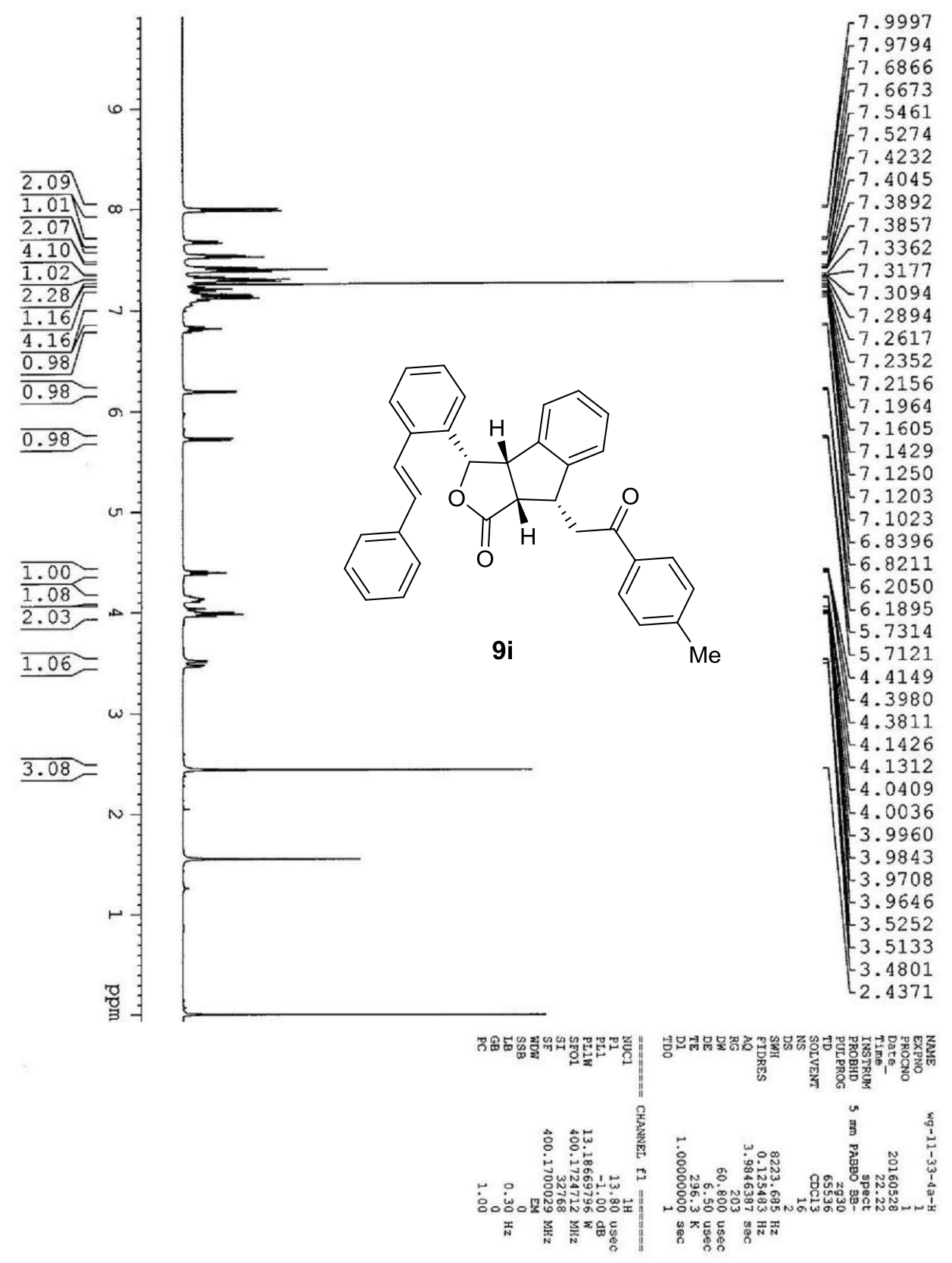




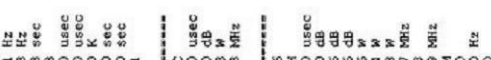

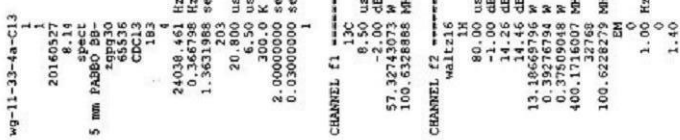

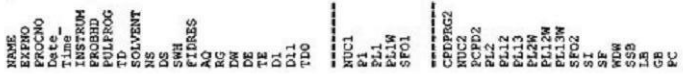

$8 \varepsilon \tau \cdot 8 \varepsilon=$

$662 \cdot L V=$

$9 S S \cdot 6 D=$

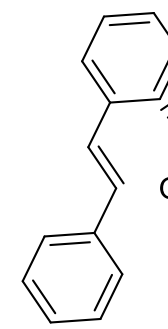

$\angle 0 L \cdot 9 L$

$S 20^{\circ} L L$

$\varepsilon \sqcup \varepsilon \cdot L L]$
$L O \varepsilon \cdot 6 L$

$08 L \cdot 2 Z T$

$\angle Z G^{\circ} \nabla Z I$

$9 \nabla \tau \cdot 9 Z \tau$

$Z L \varepsilon \cdot 9 Z \tau$

9LG.9ZT

OZL.9Z .

$689^{\circ} \mathrm{LZT}$

$\varepsilon 66^{\circ} \mathrm{LZT}$

โ0Z.8Zโ

$98 \tau \cdot 8 \tau \tau$

$08 \pi^{\circ} 82 \tau$

$968 \cdot 82 \tau$

$\varepsilon \mathrm{G} \cdot 6 Z \tau$

โ09. $2 \varepsilon \tau /$ )

$805^{\circ} \varepsilon \varepsilon \tau$

$\varsigma 99^{\circ} \circ \varepsilon \tau$

ऽ $0^{\circ} \mathrm{SET}$

โร $6^{\circ} 9 \varepsilon \tau$.

9 乙ह ${ }^{*} L \varepsilon \tau$

$\varepsilon \varepsilon L \cdot \varepsilon \nabla \tau$

โOE. $S \nabla \tau$

$80 \varepsilon^{\circ} 9 L \tau$

$\mathrm{SZ}^{\circ} 86 \mathrm{I}-$
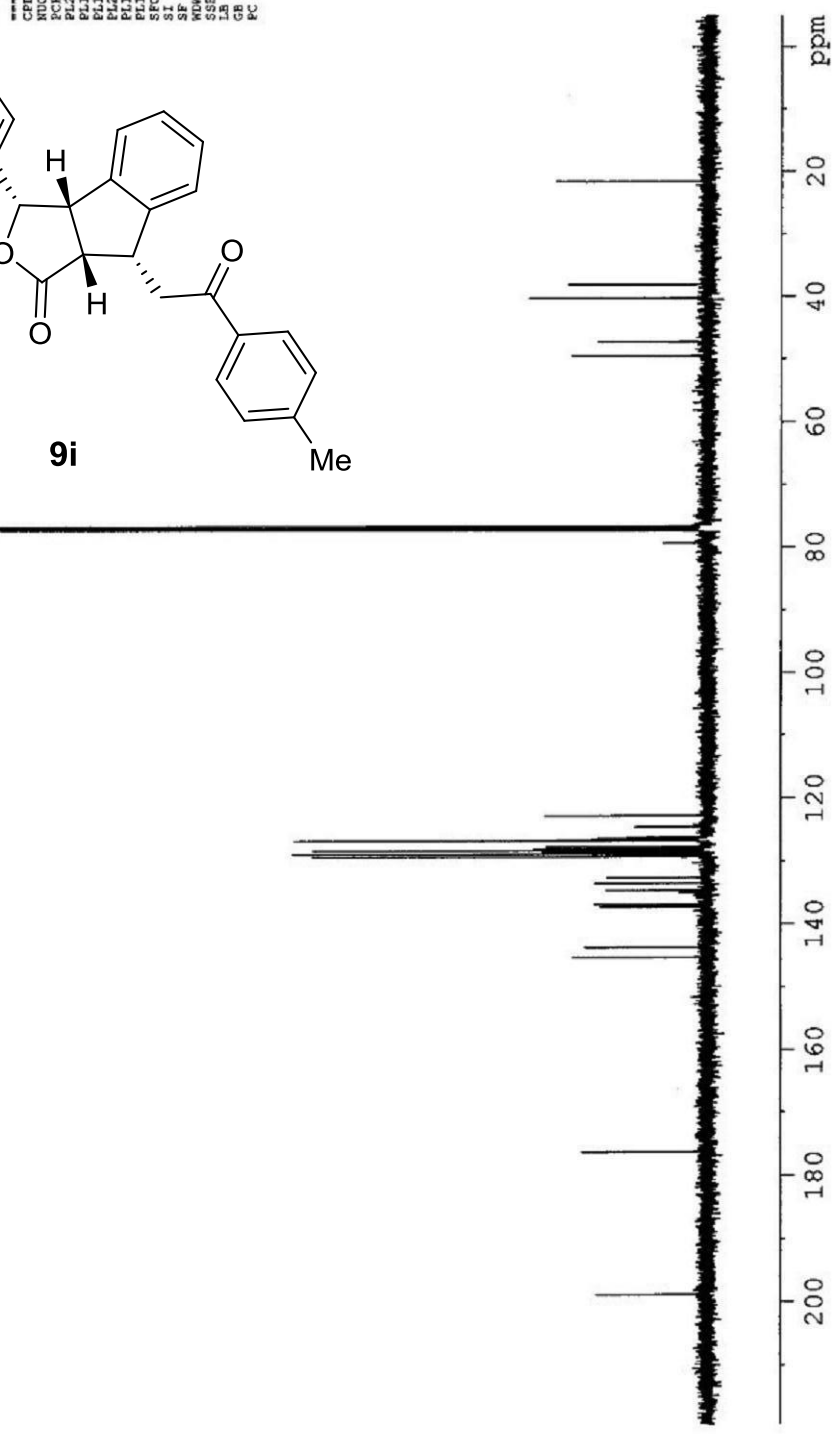


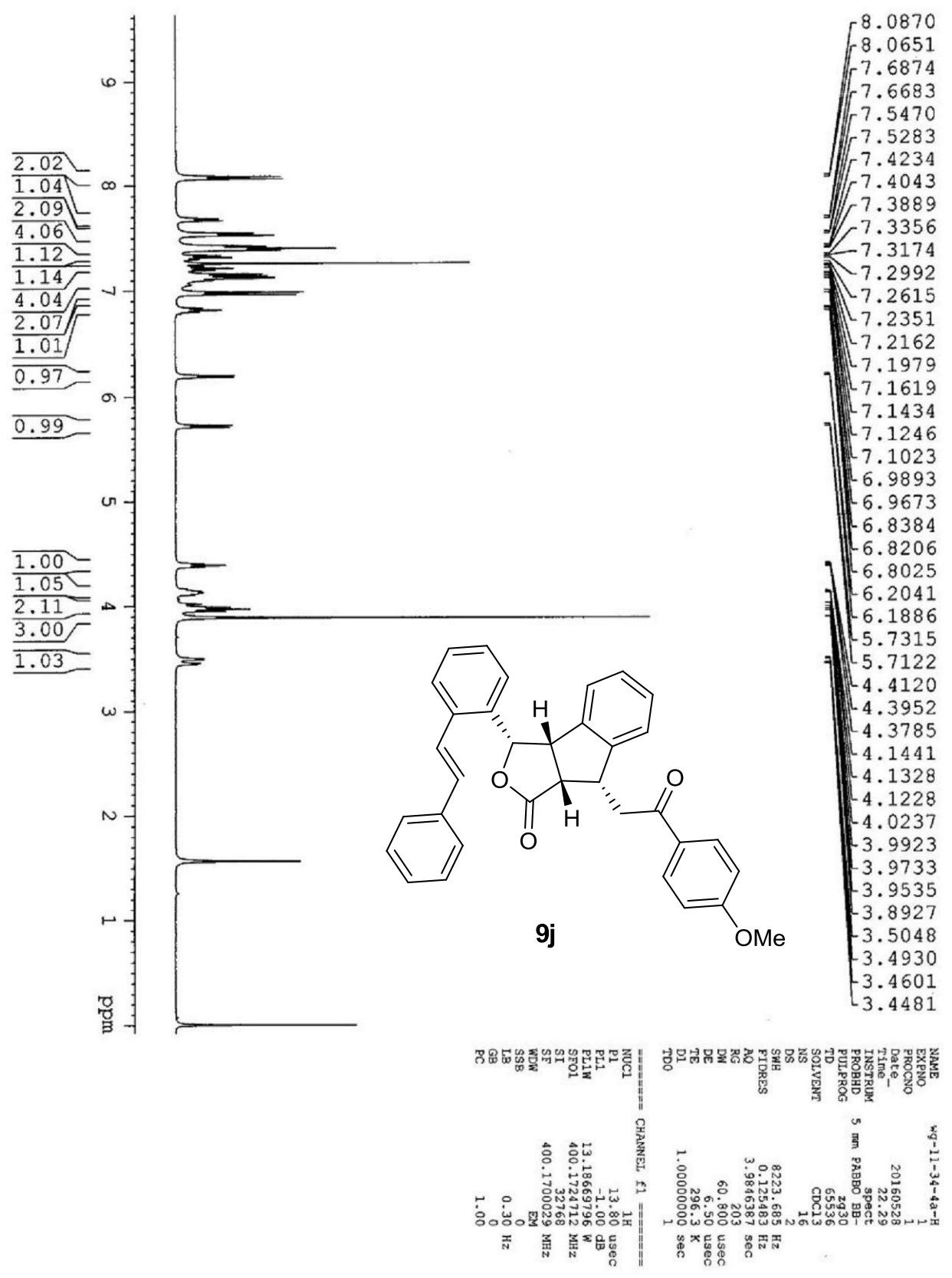




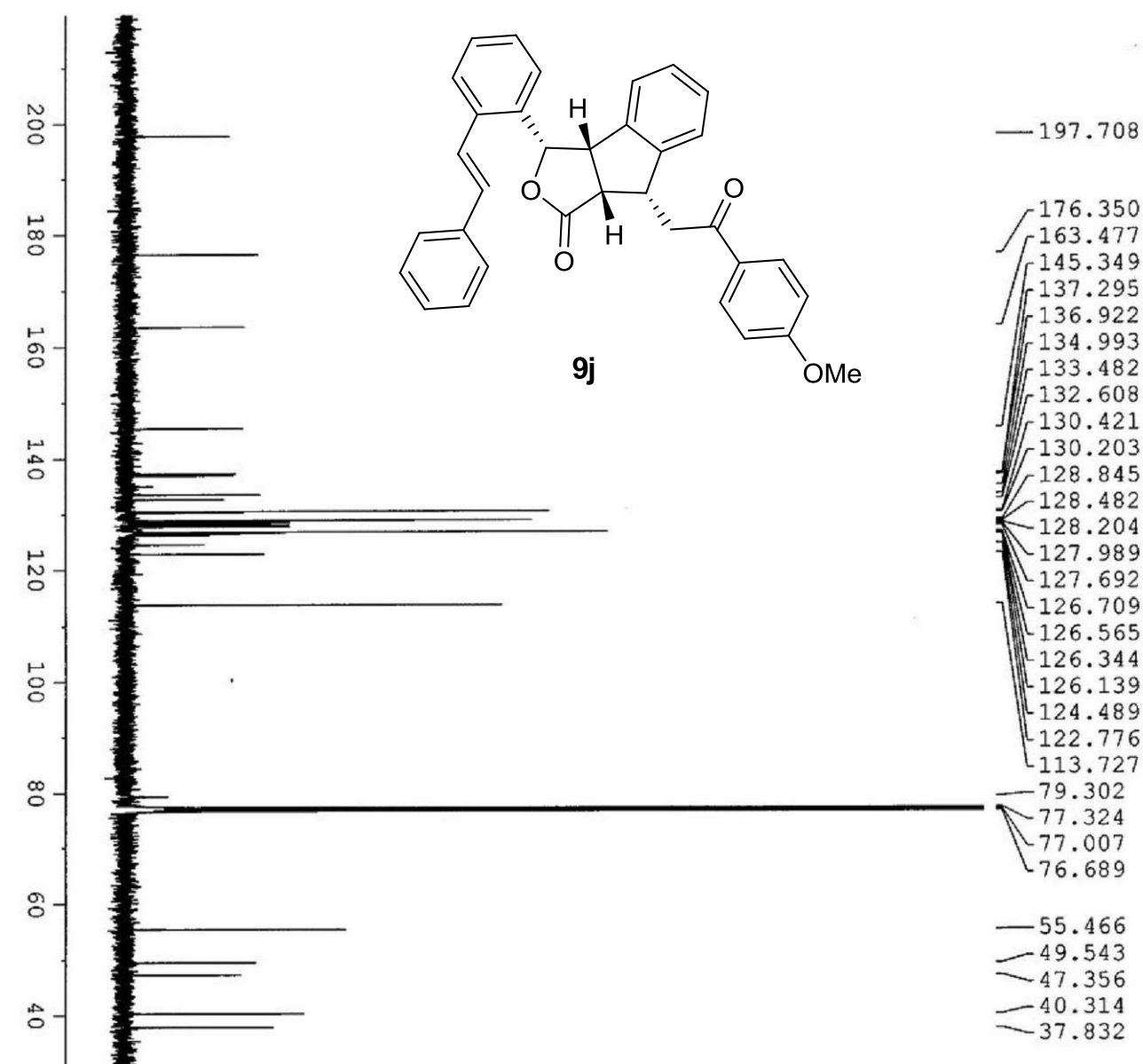

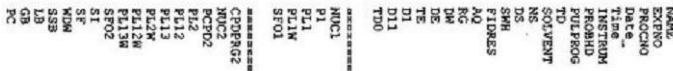

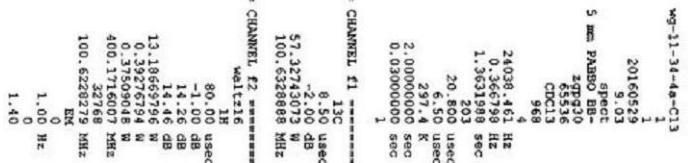




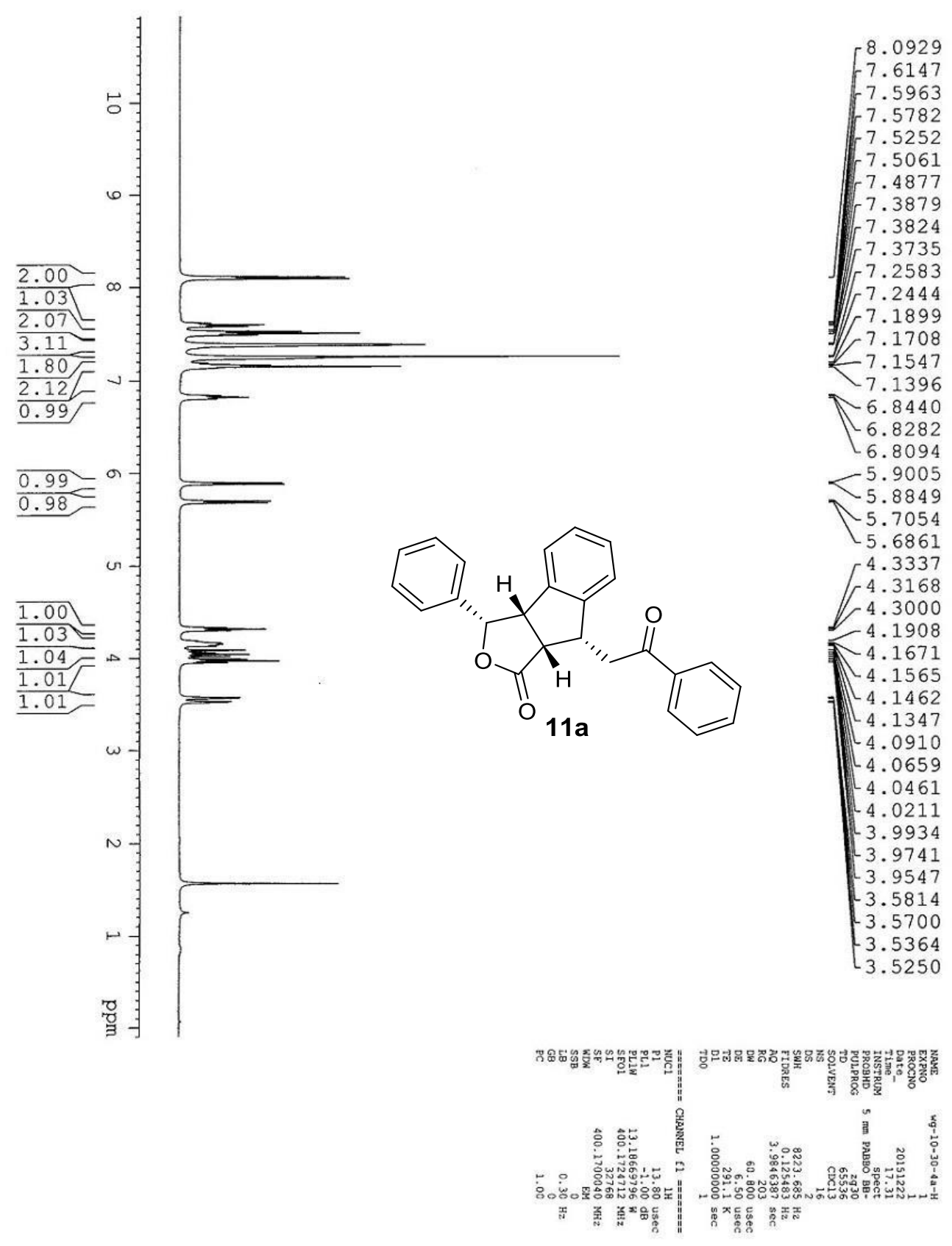




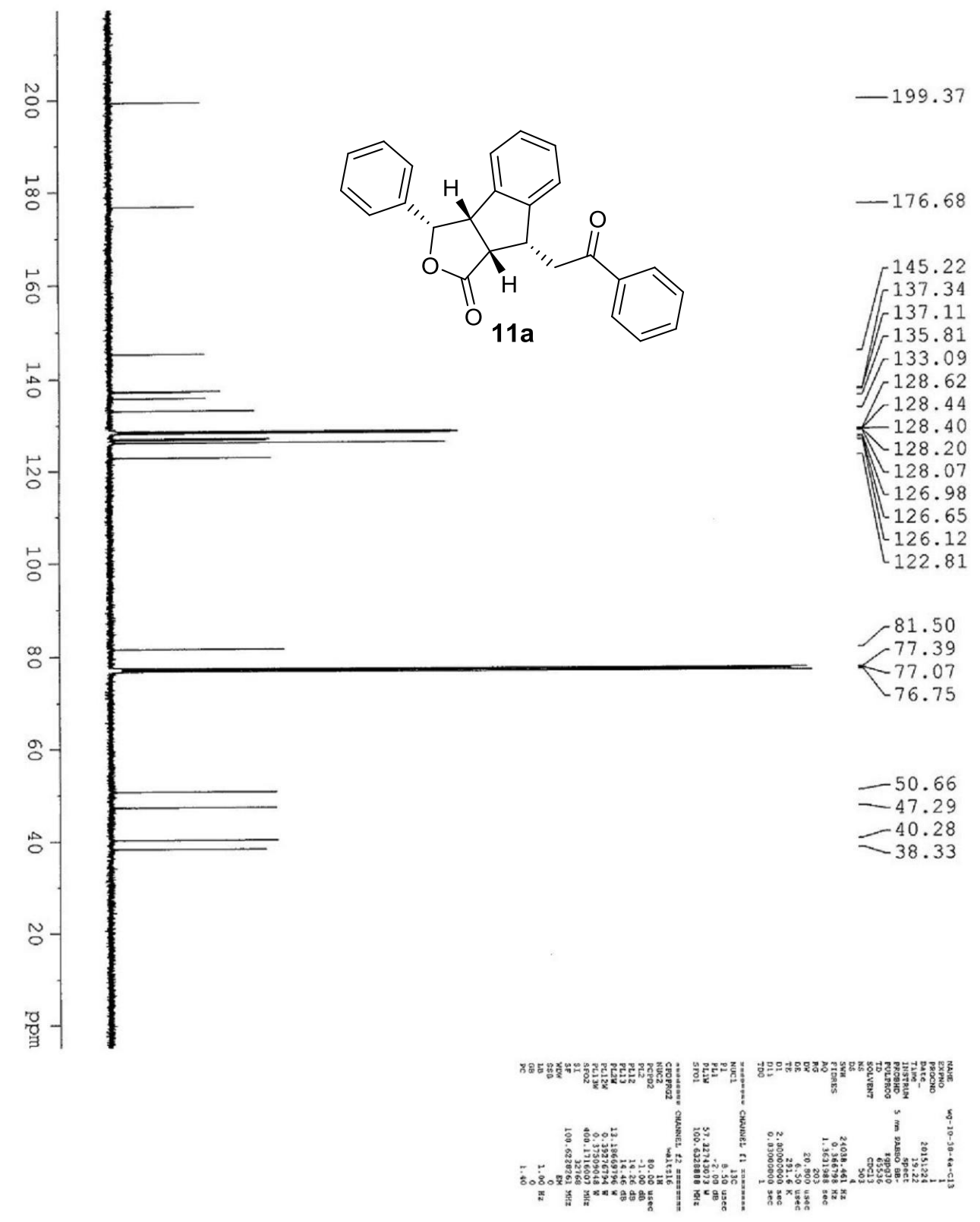




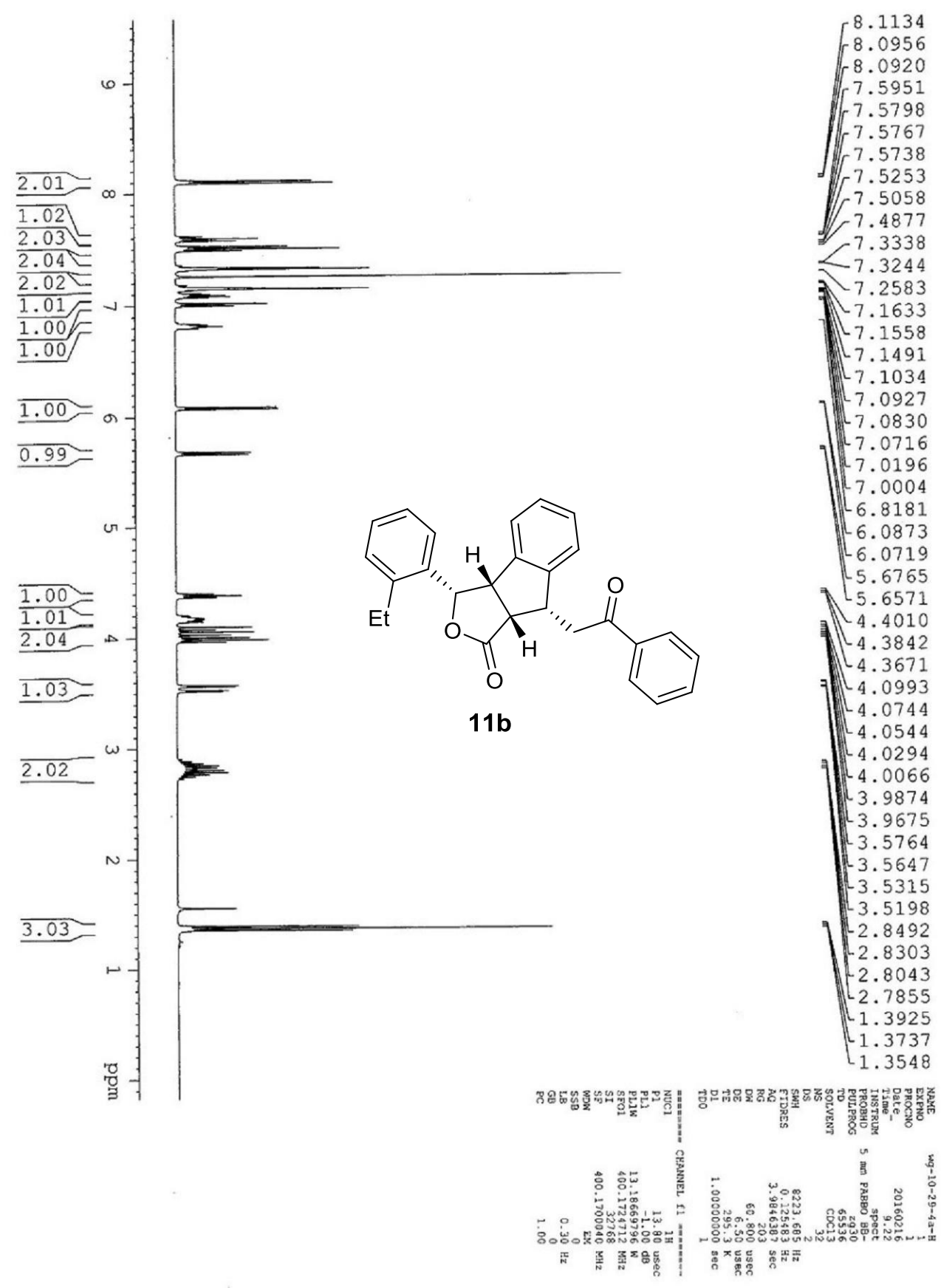




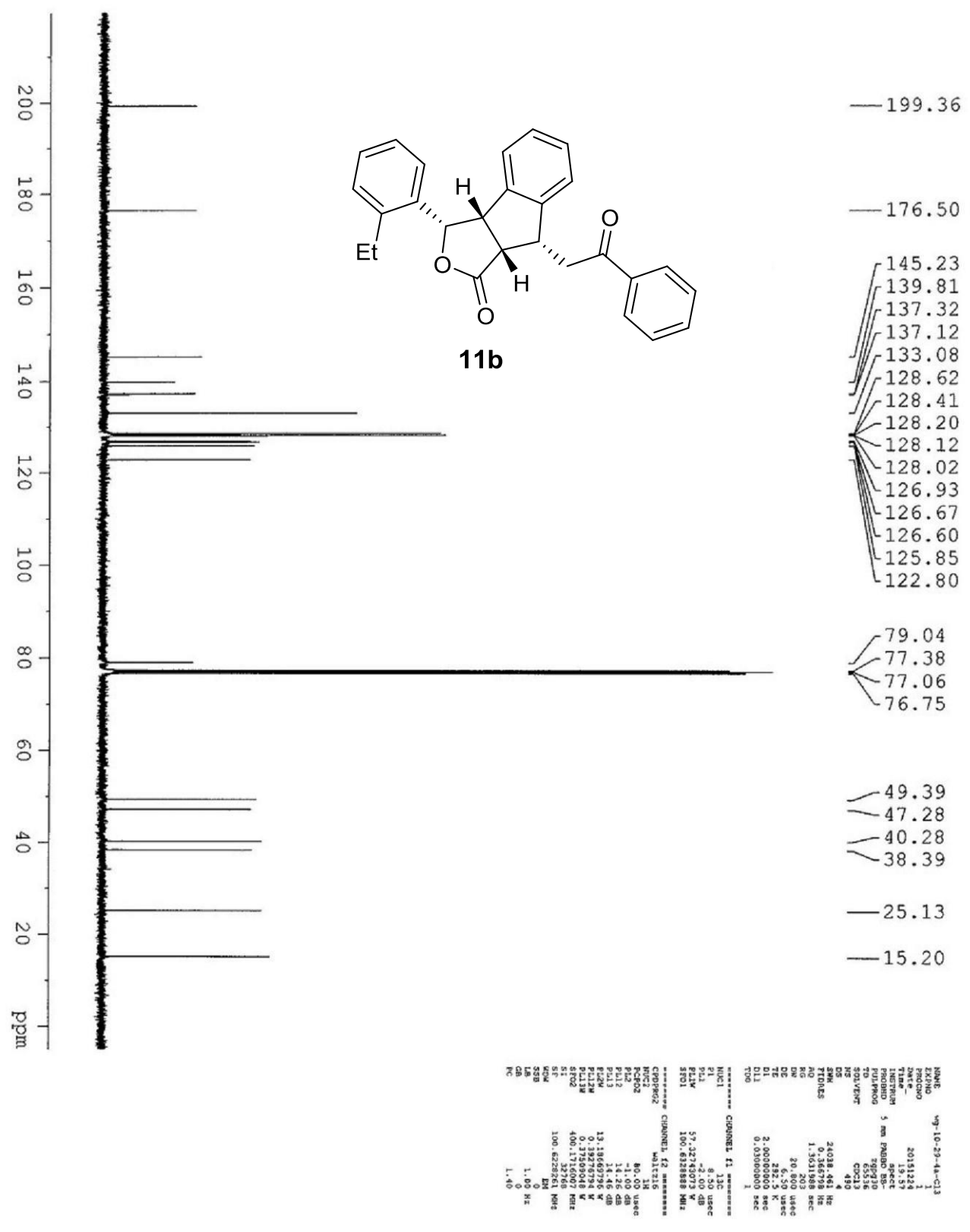




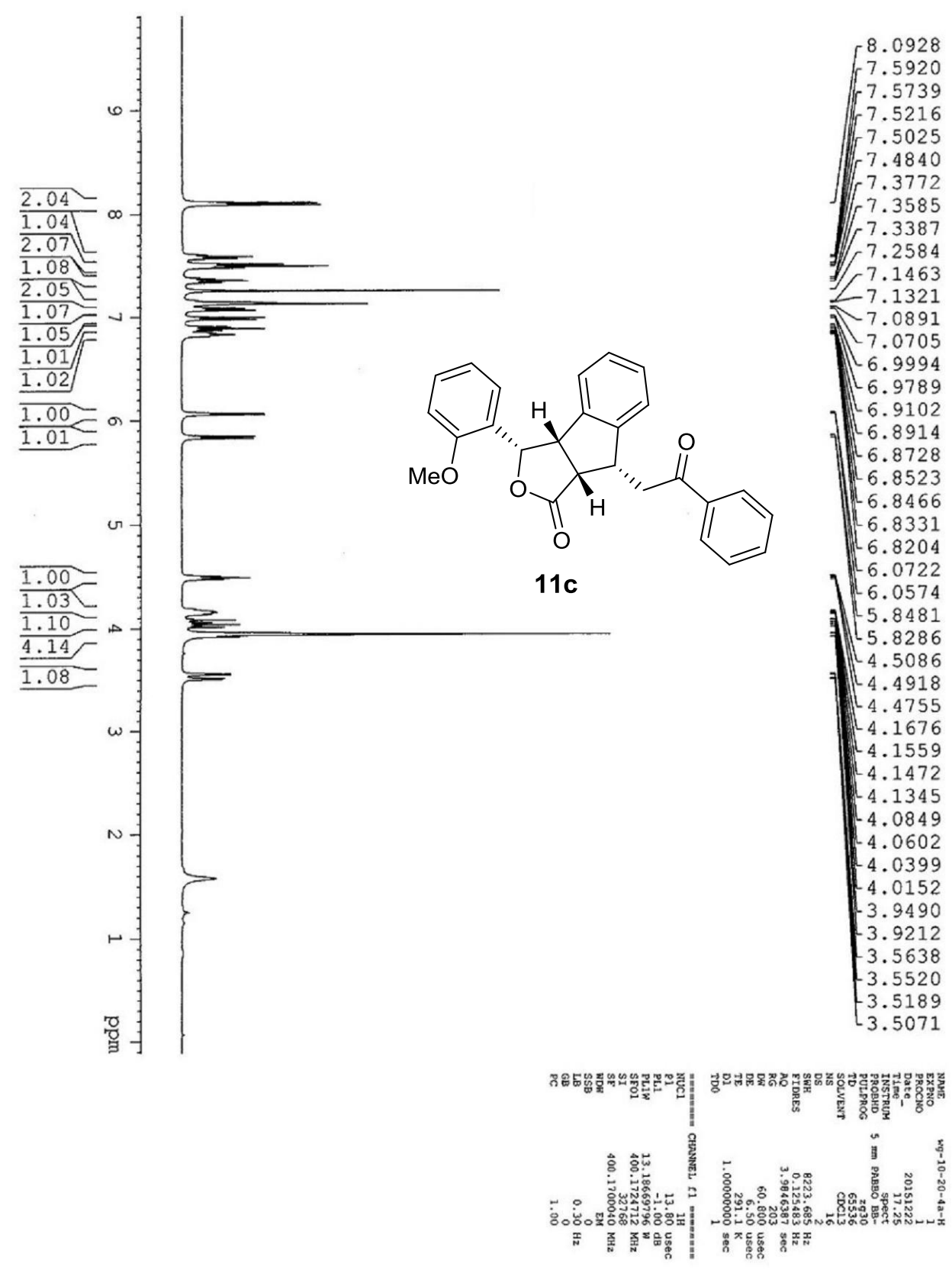




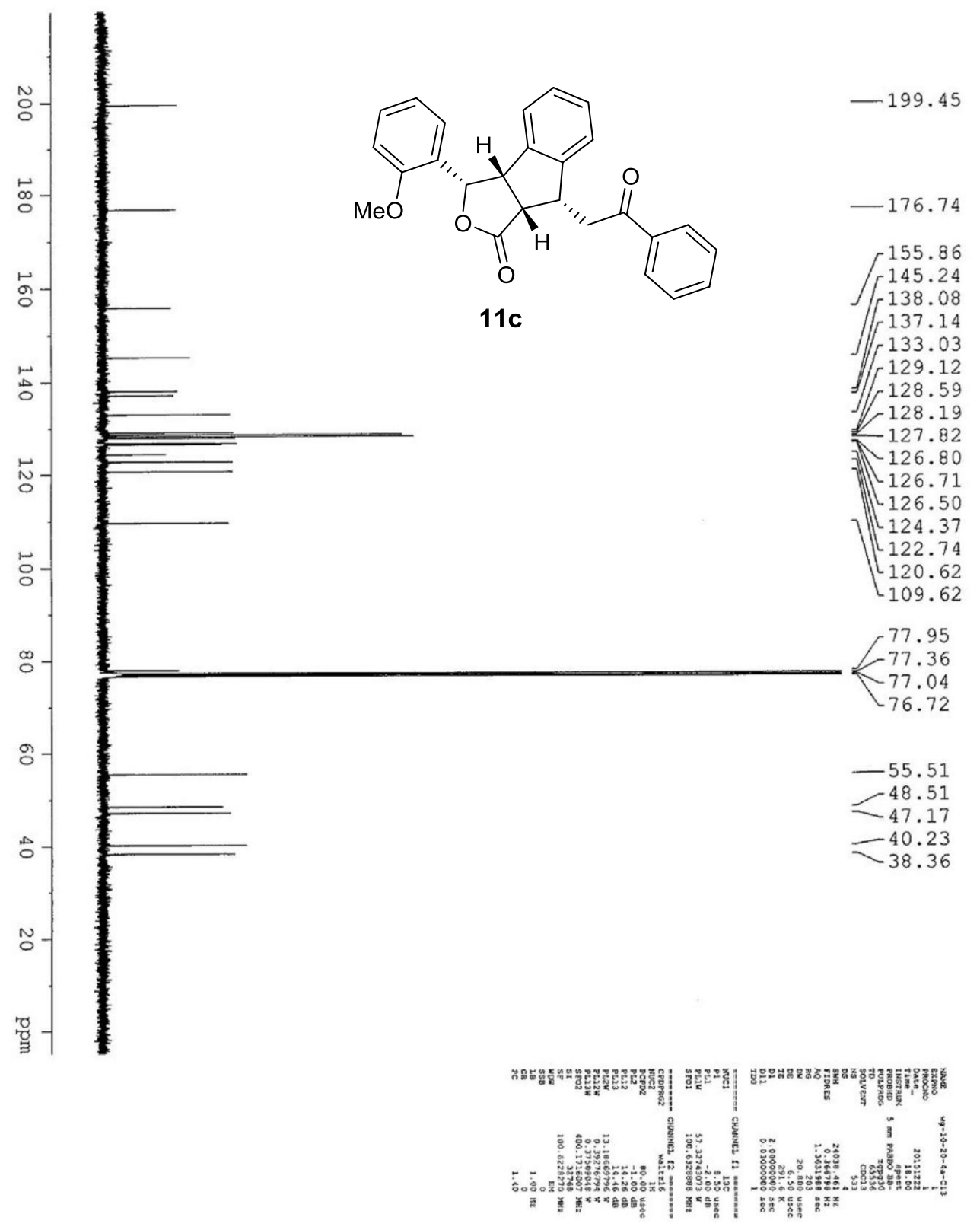




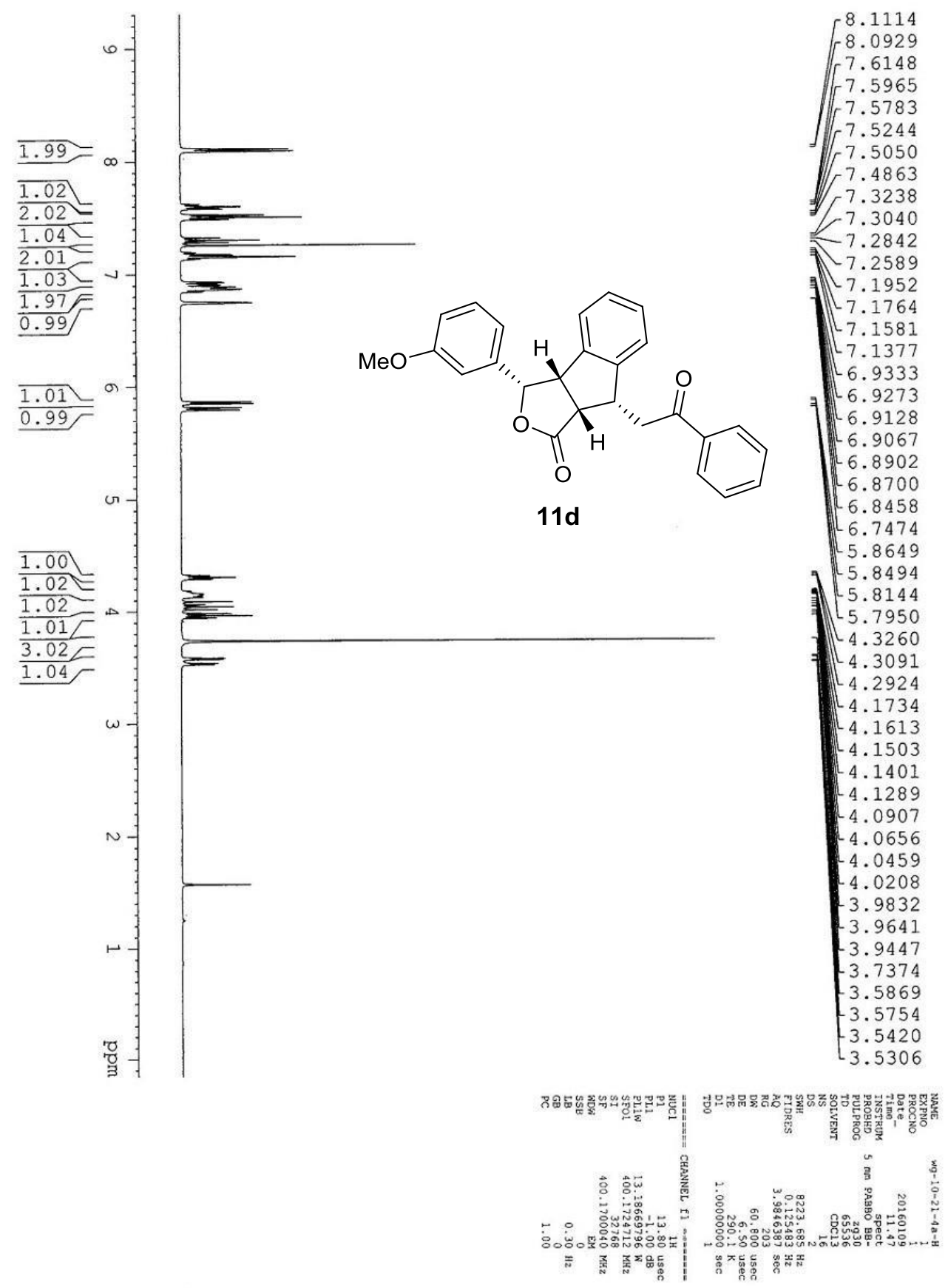




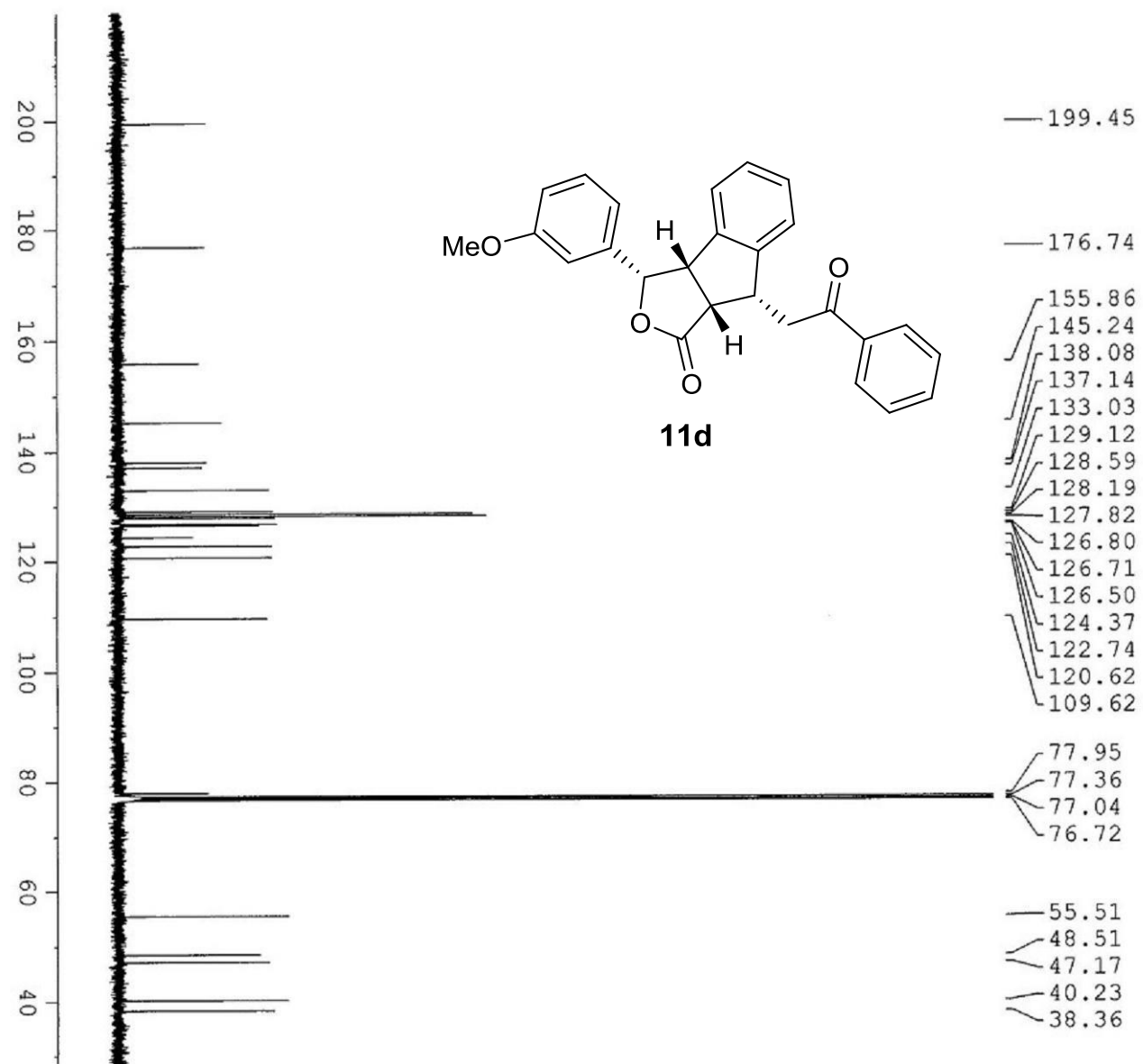

หัง

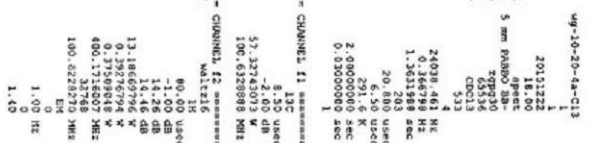




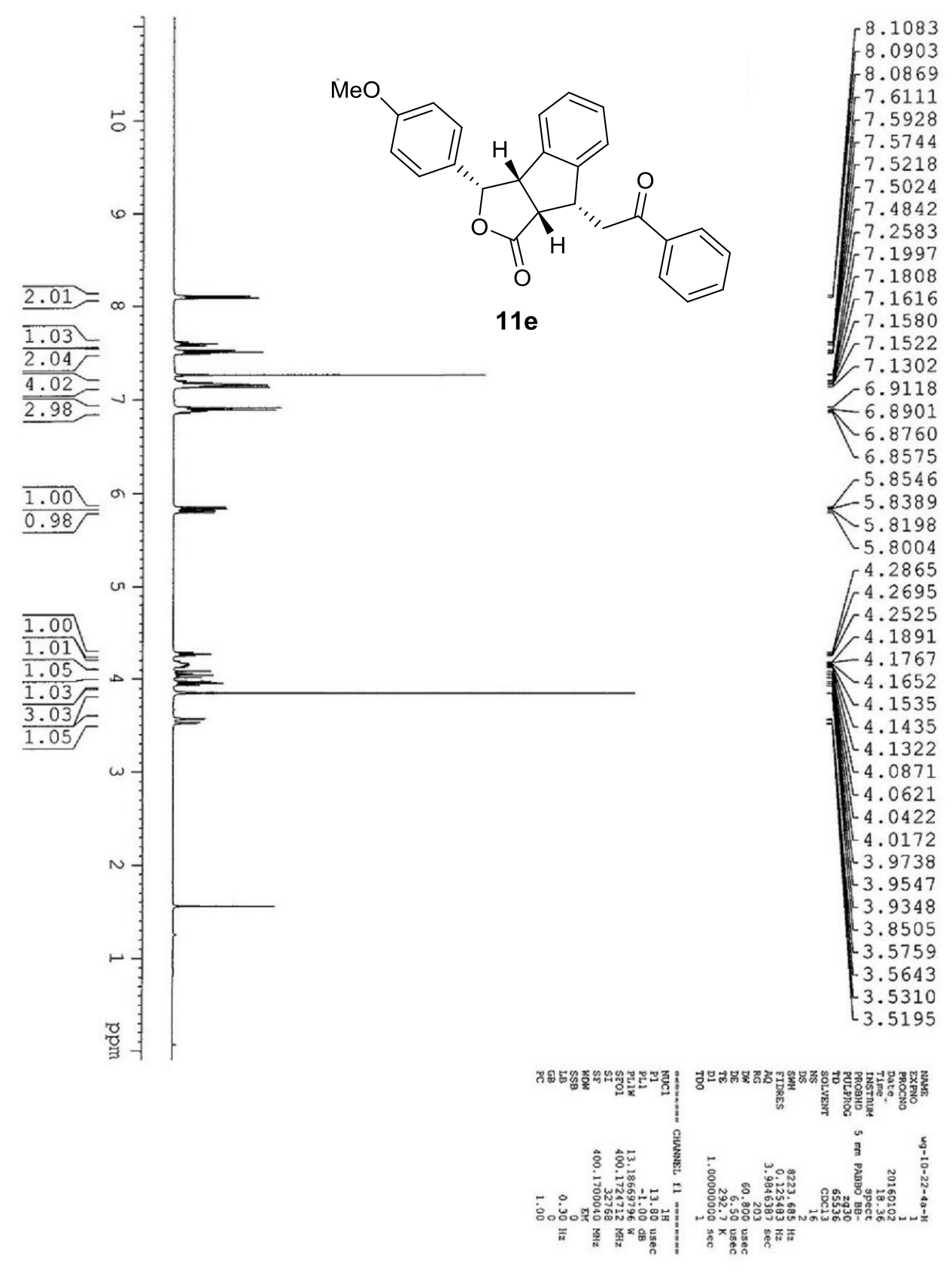




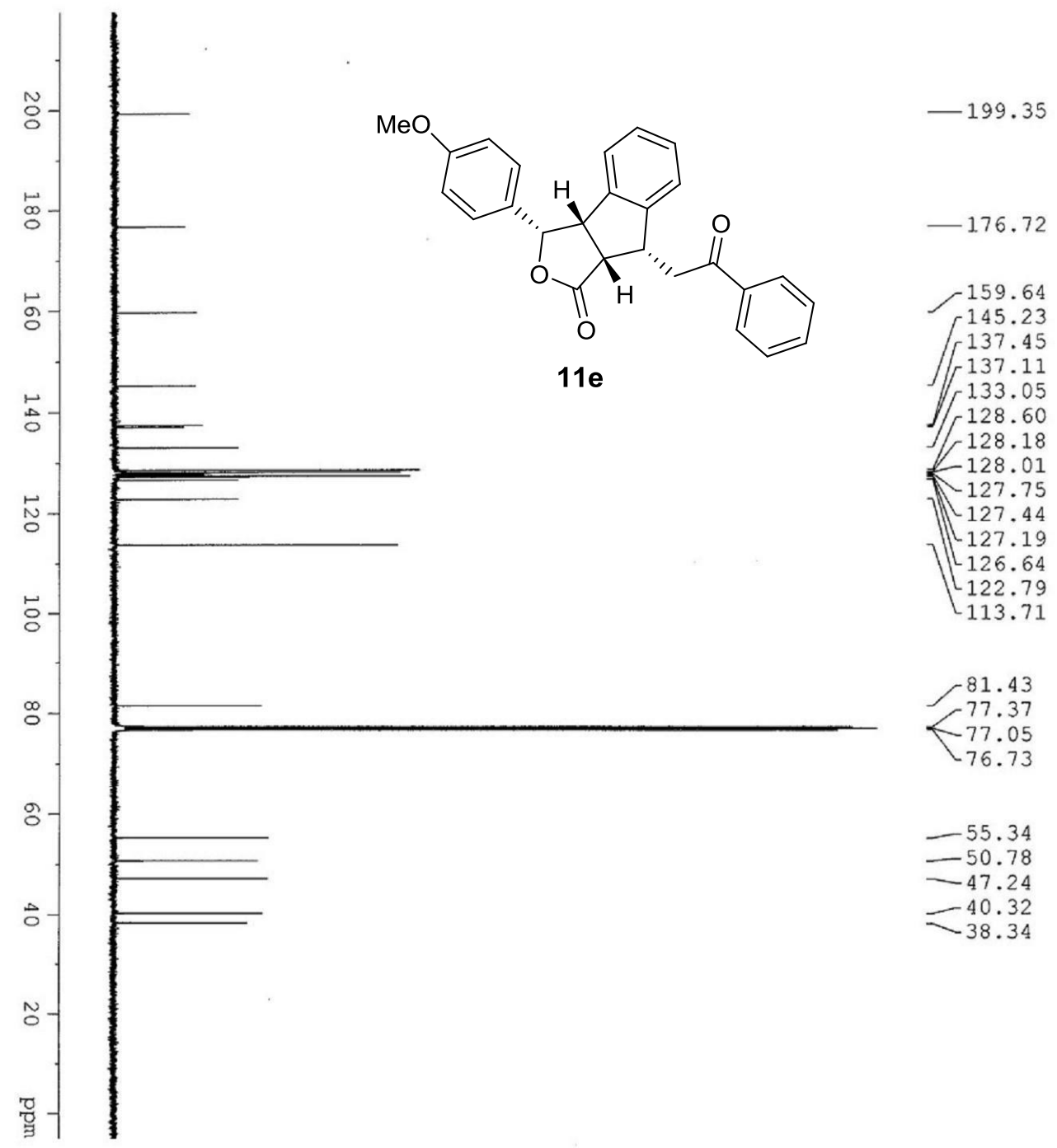

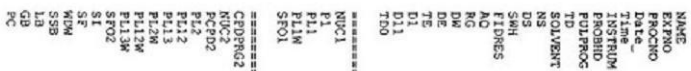

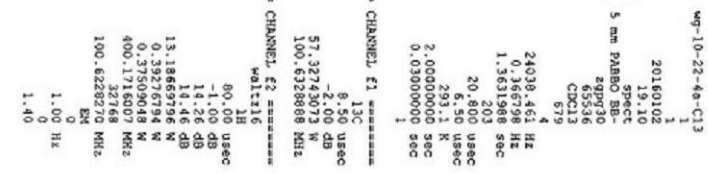




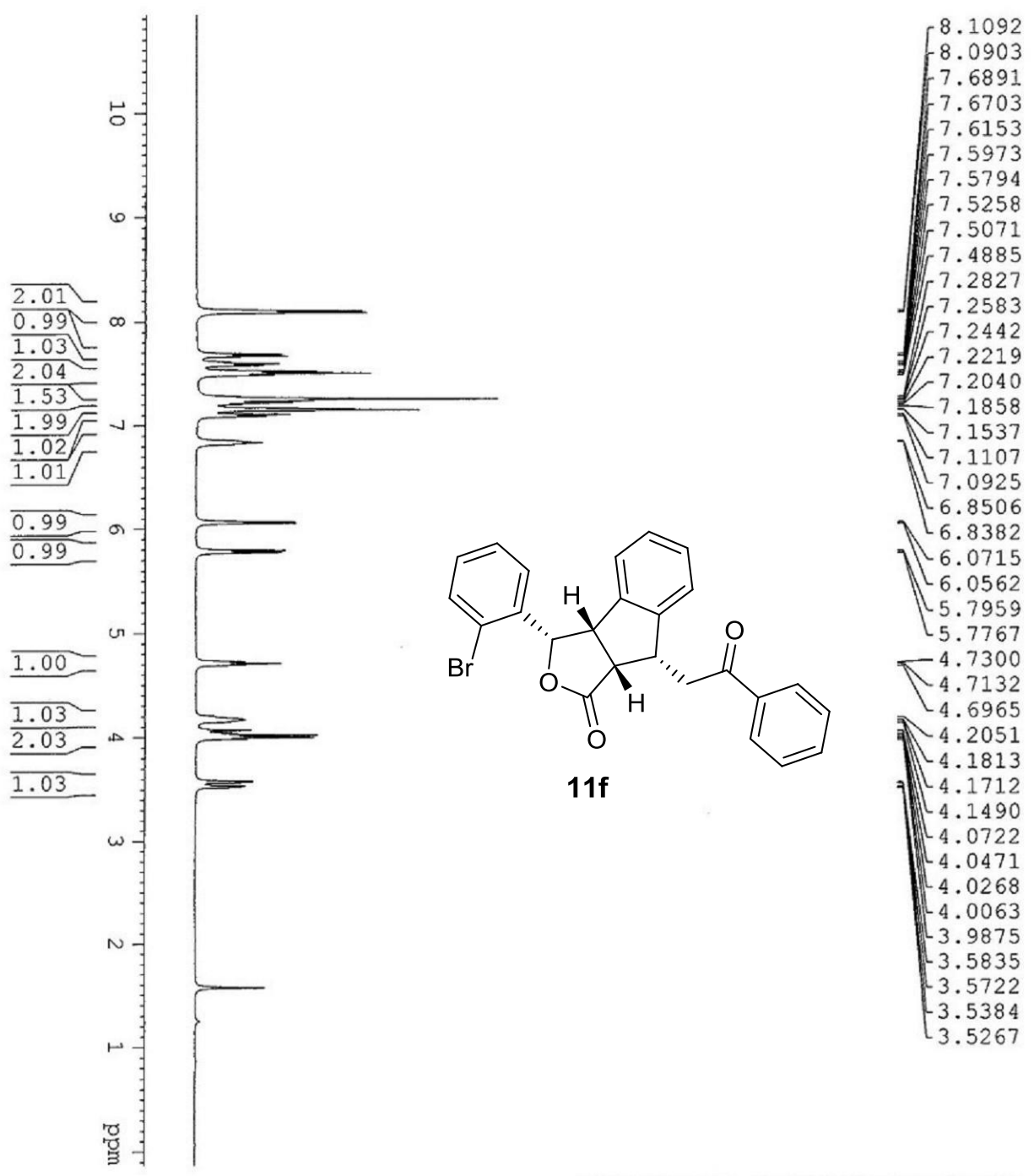

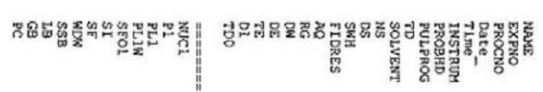

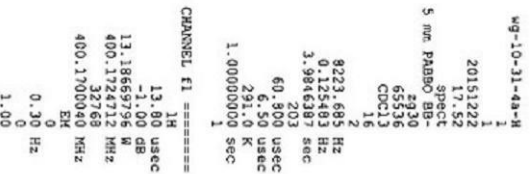




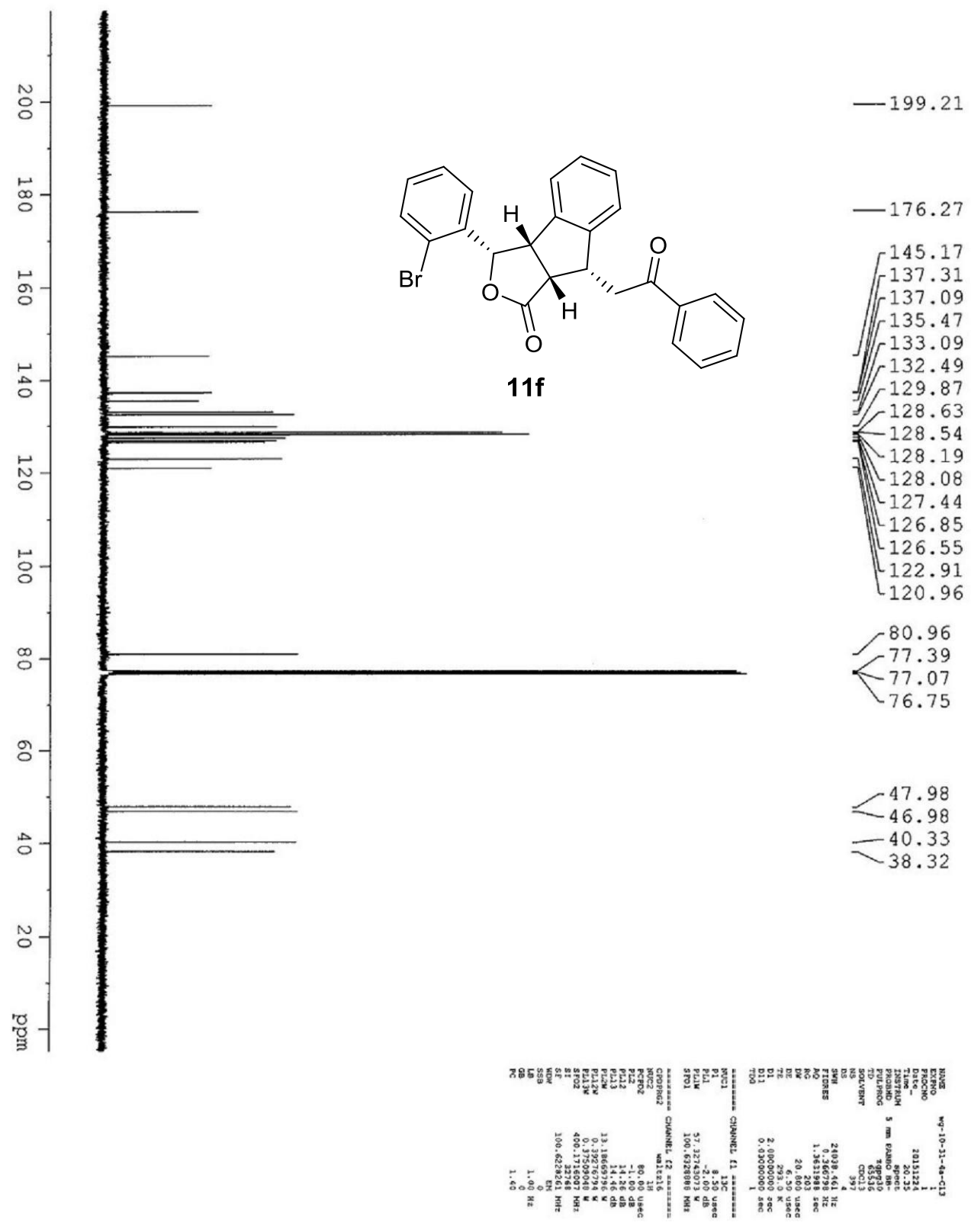




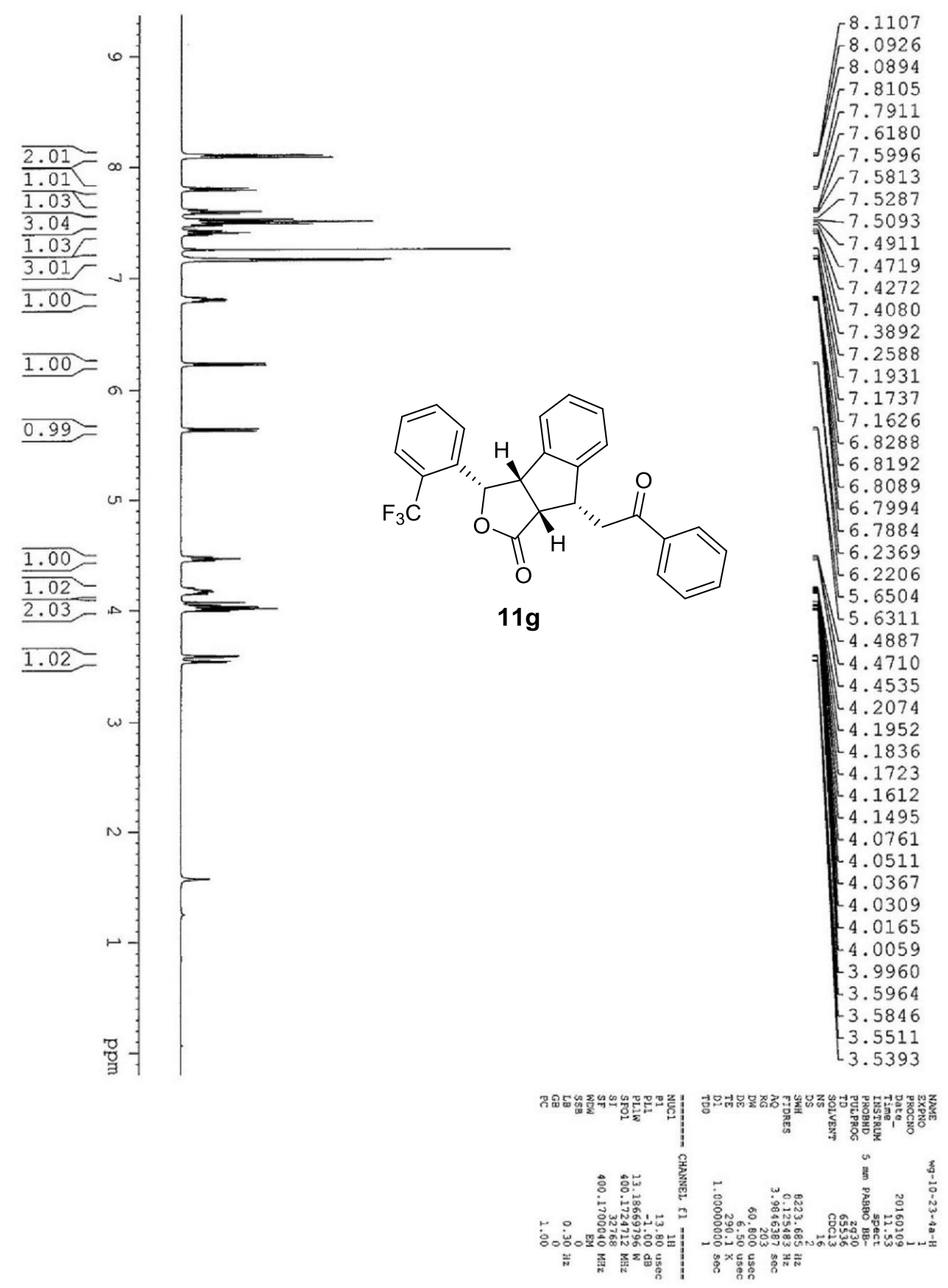




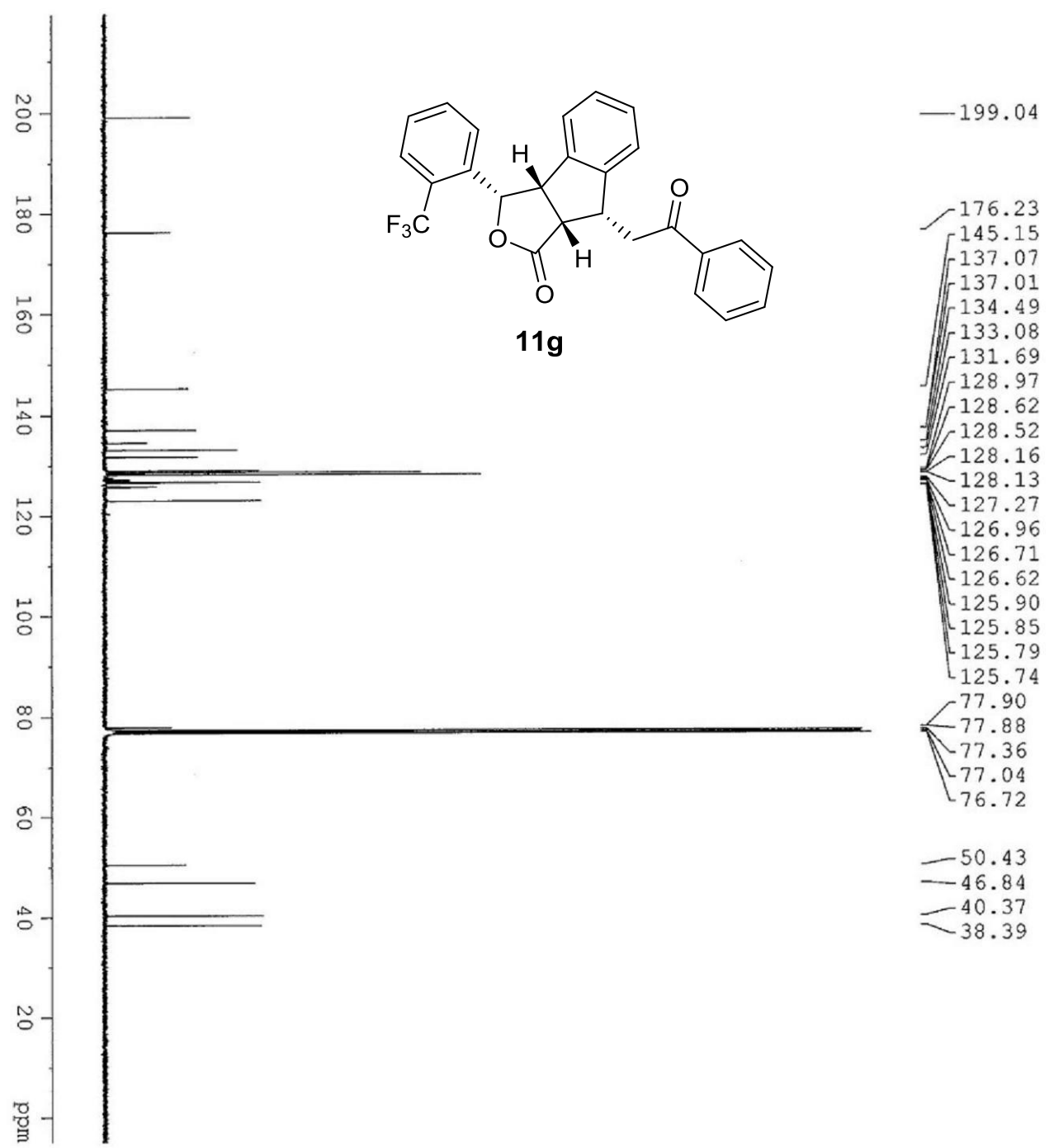

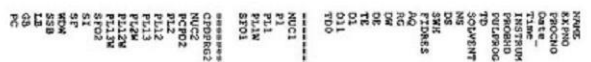

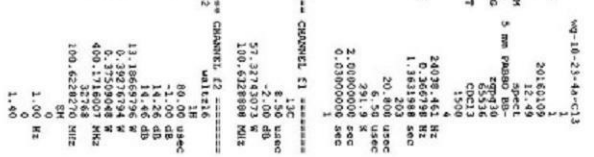




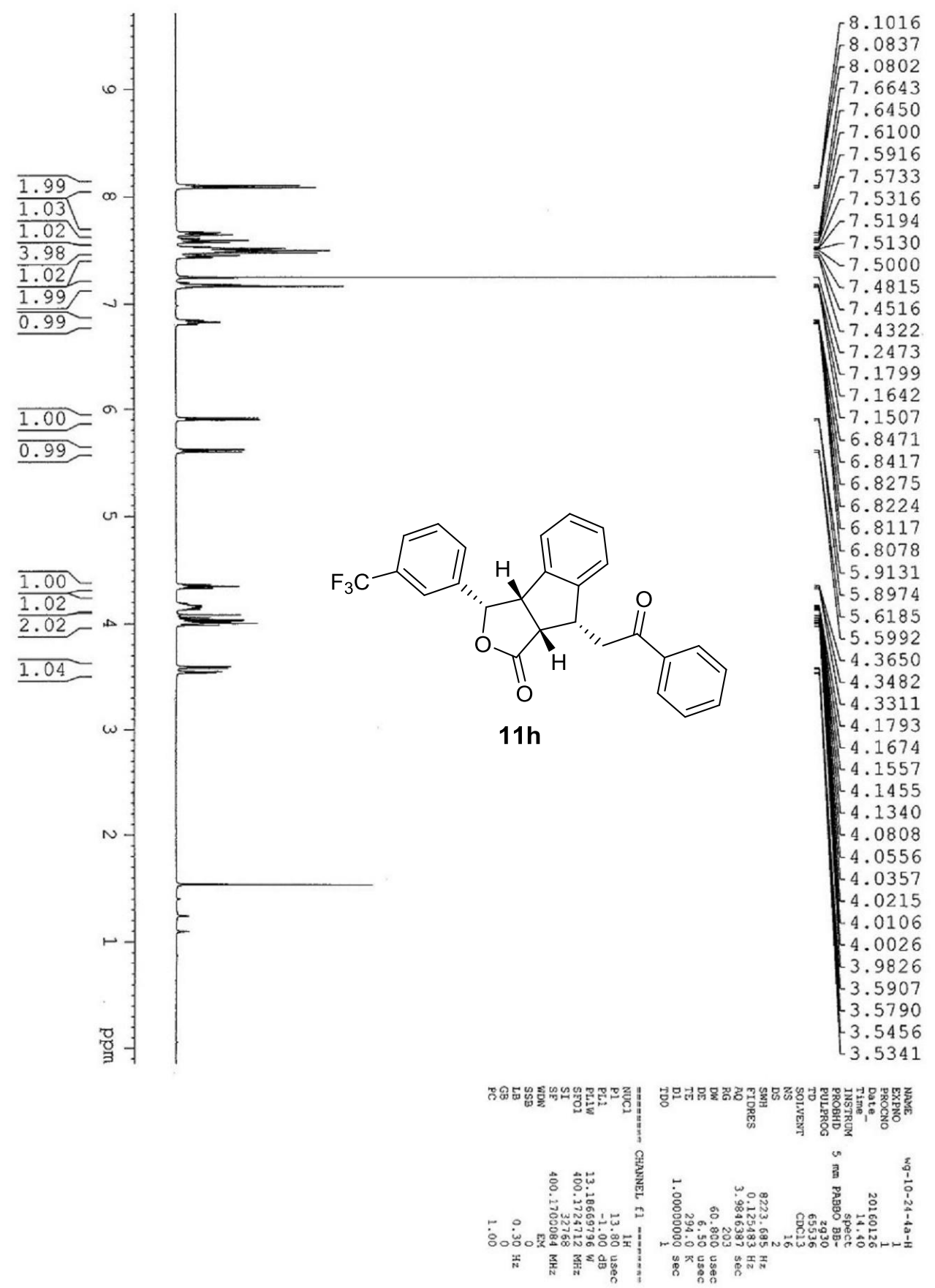




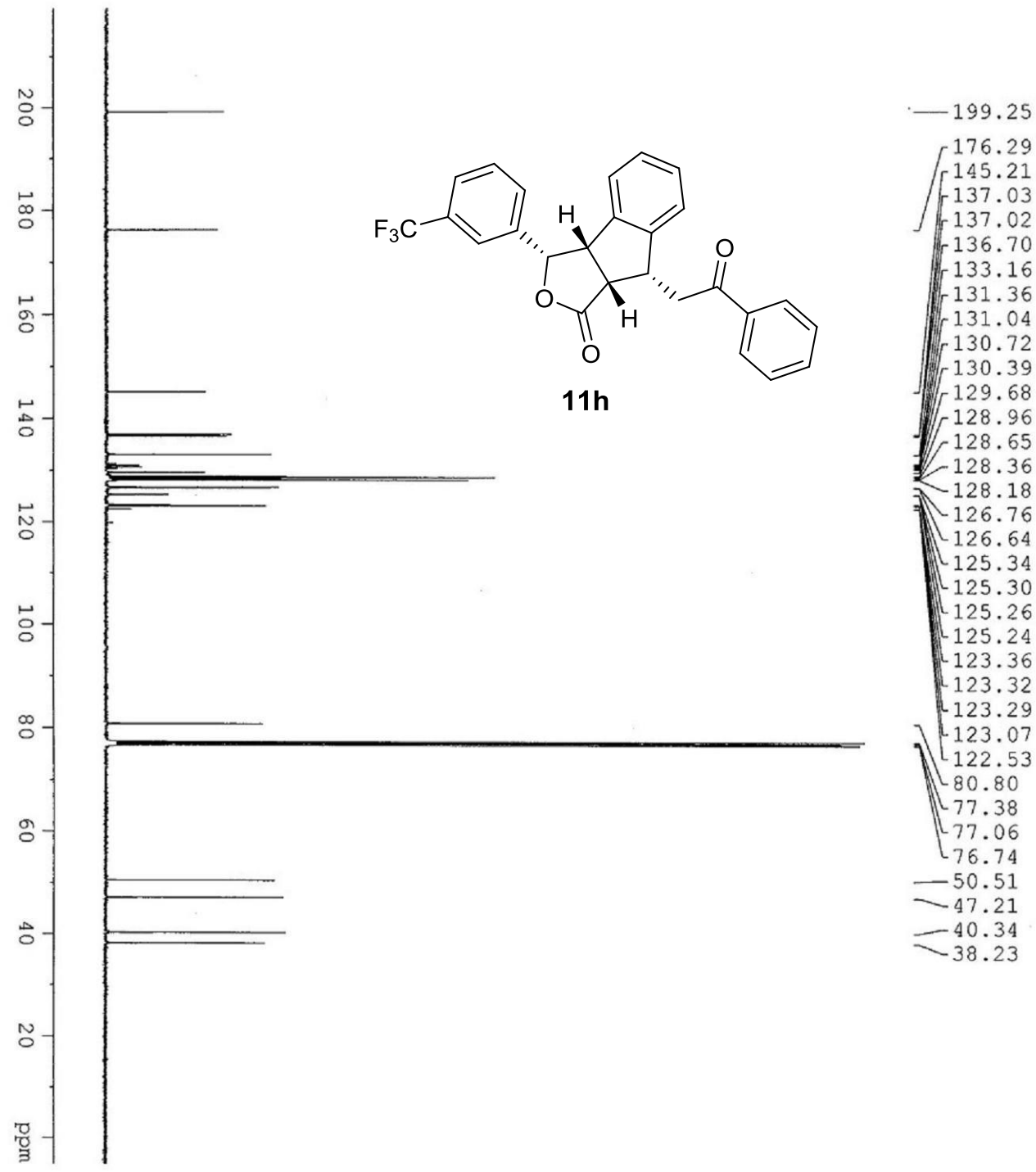

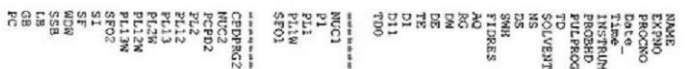

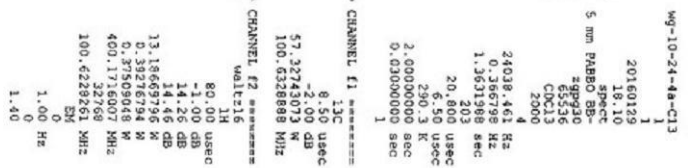




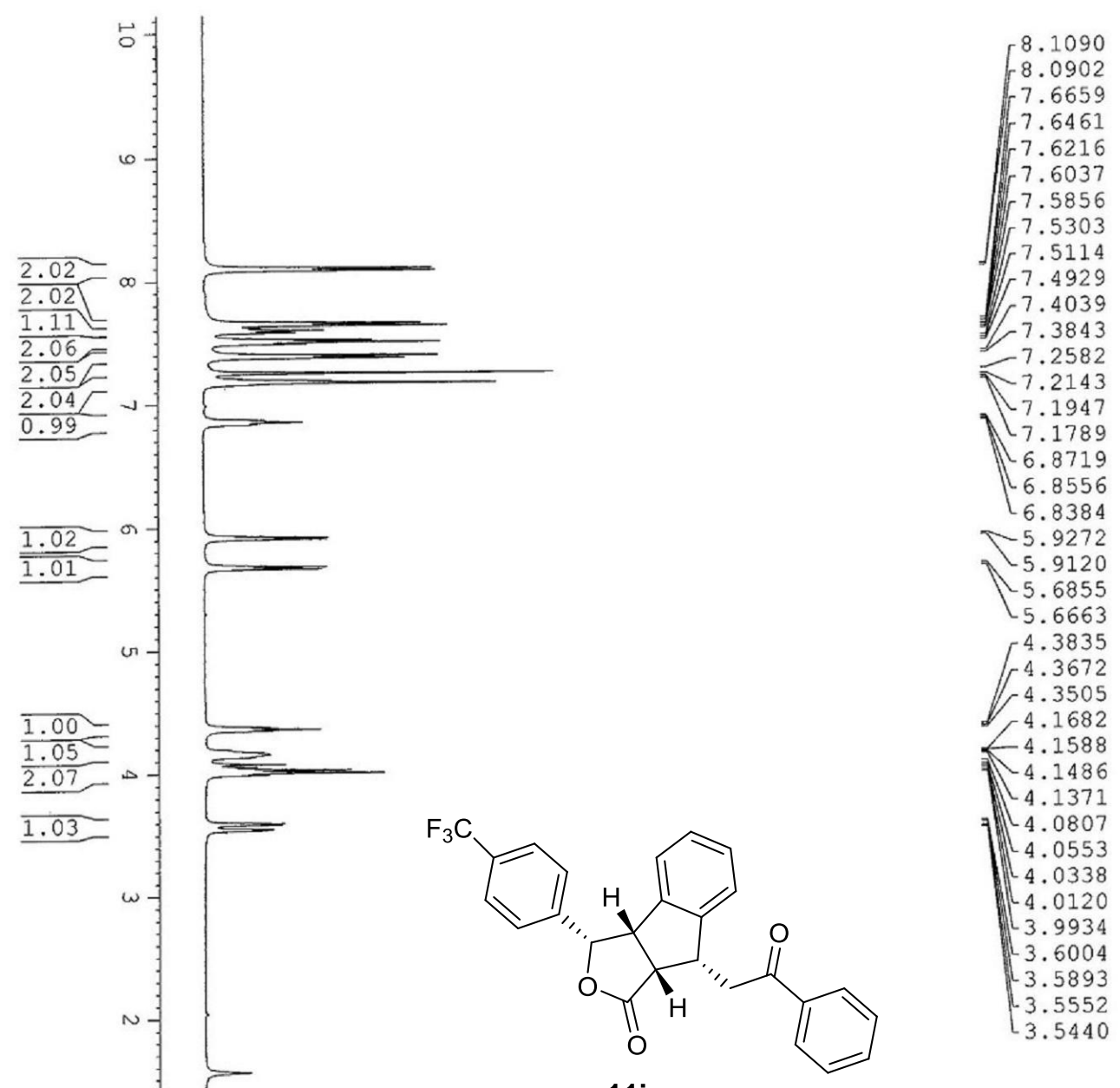

$11 \mathrm{i}$

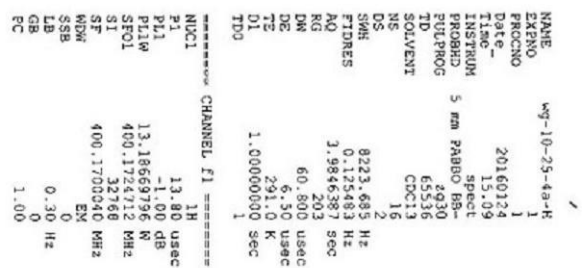




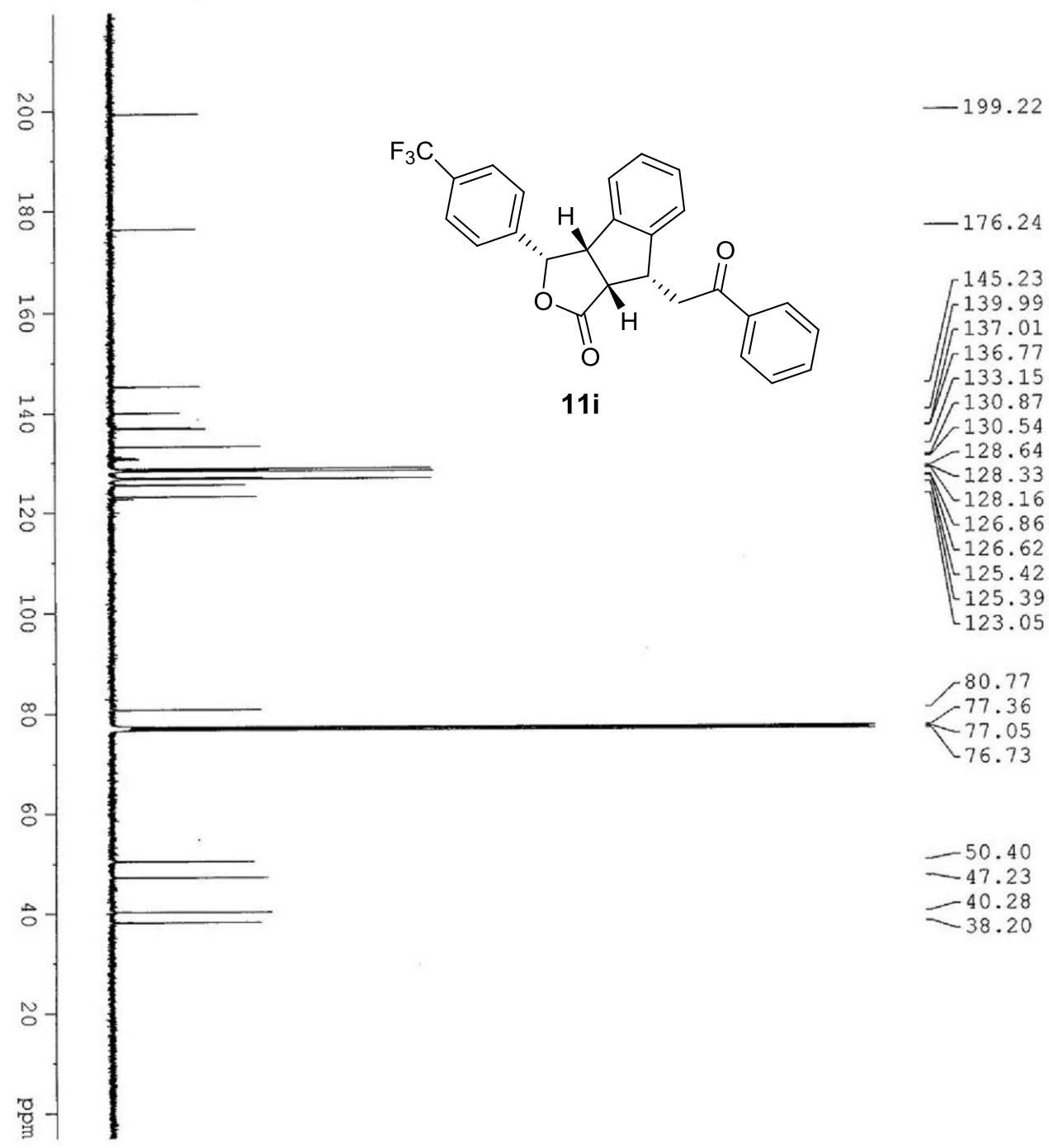

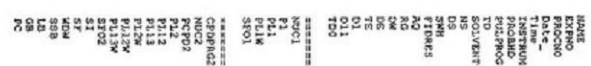

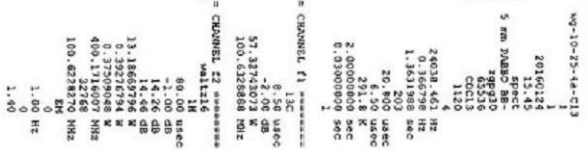



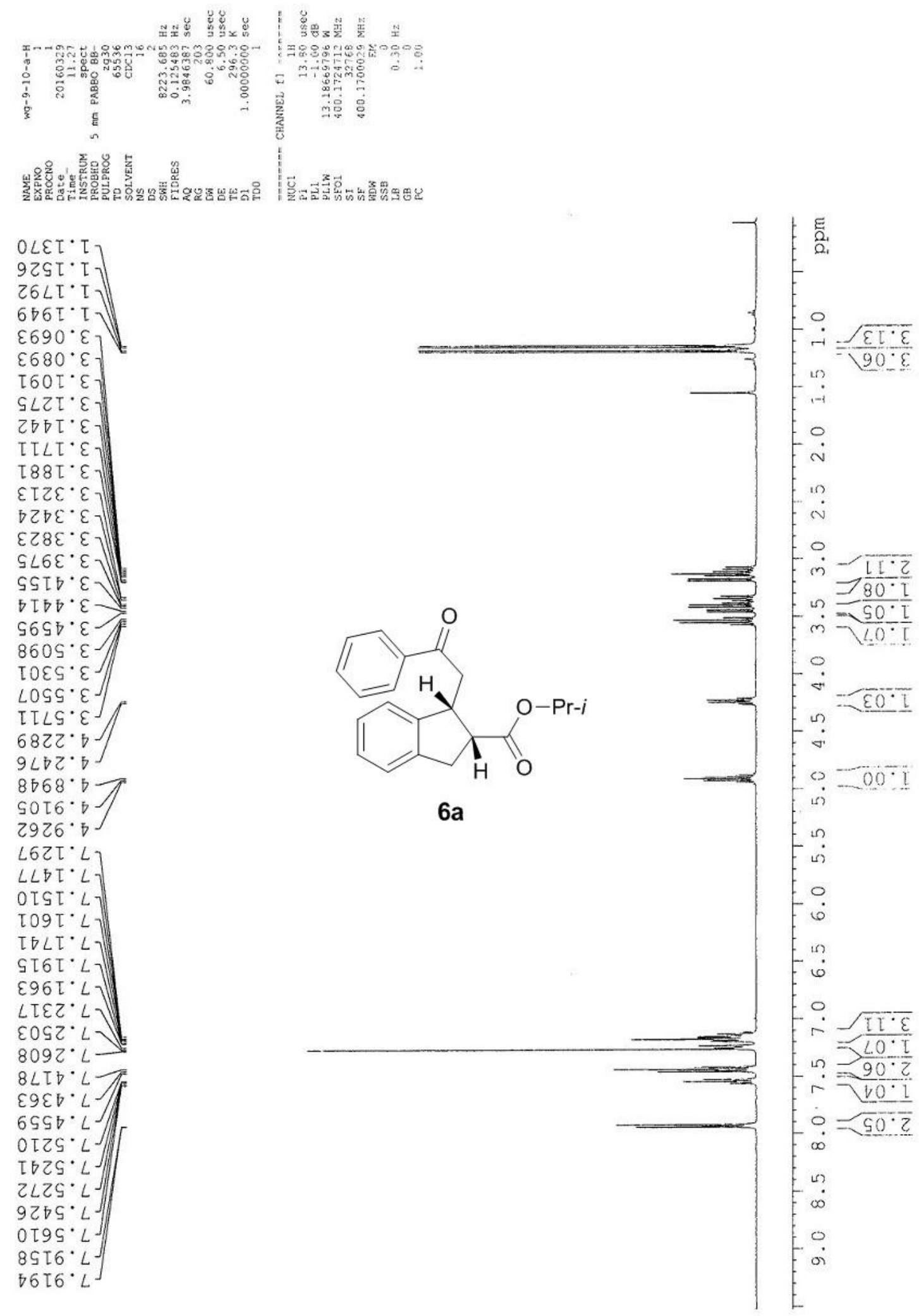

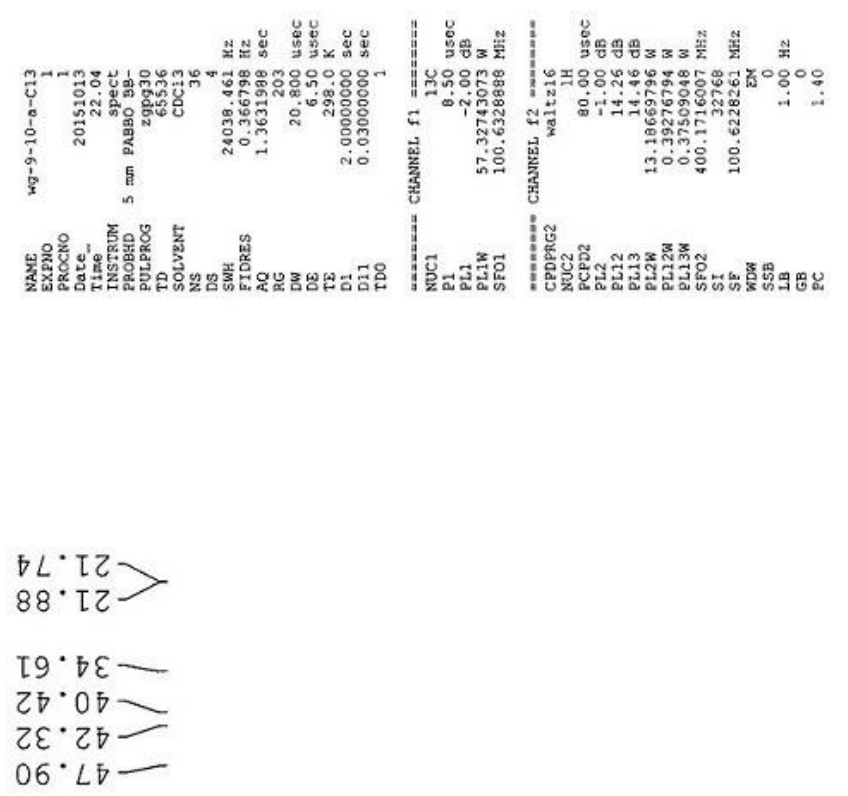

T0.89$28^{\circ} 9 L$
$51 . L L$
$96^{\circ} L L$

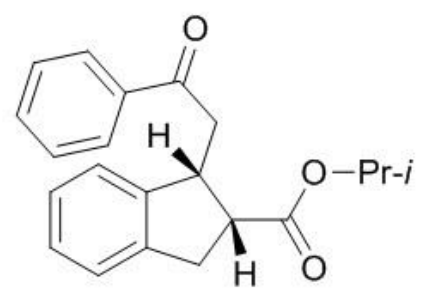

$6 a$

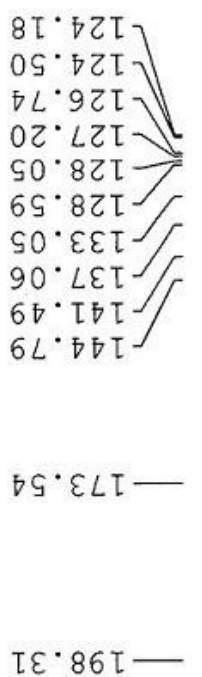

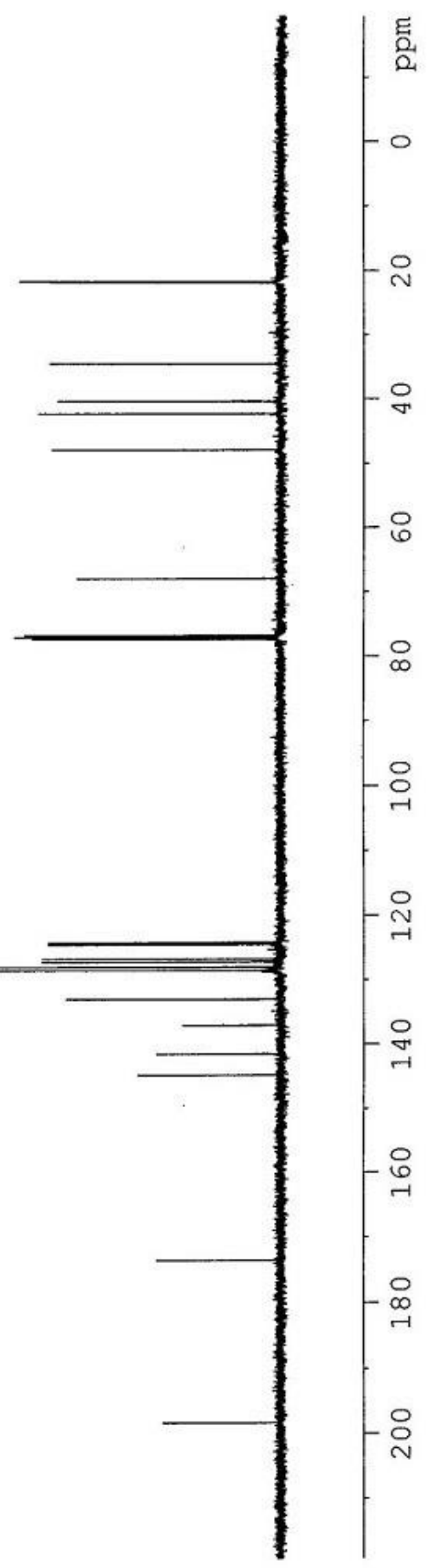




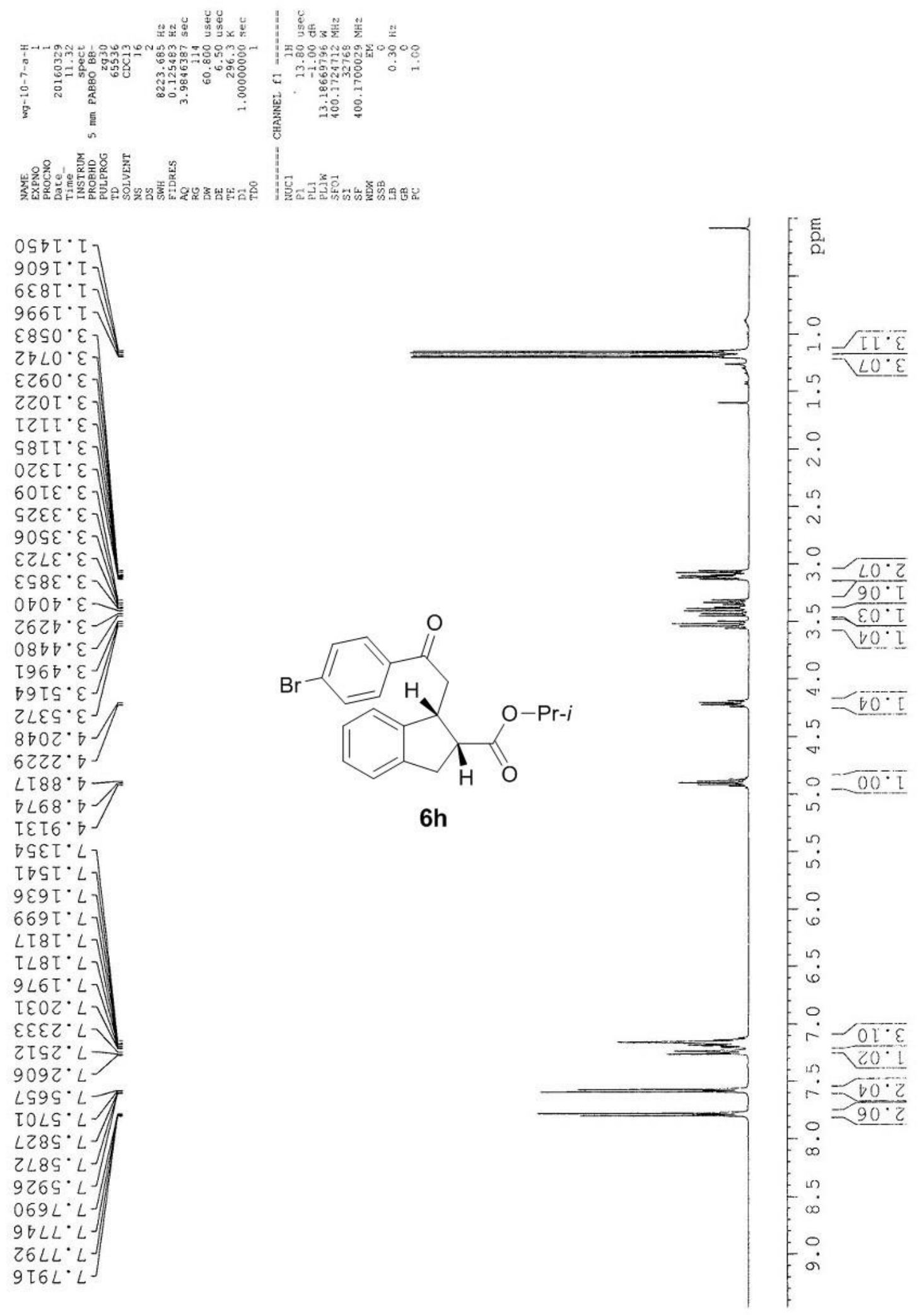




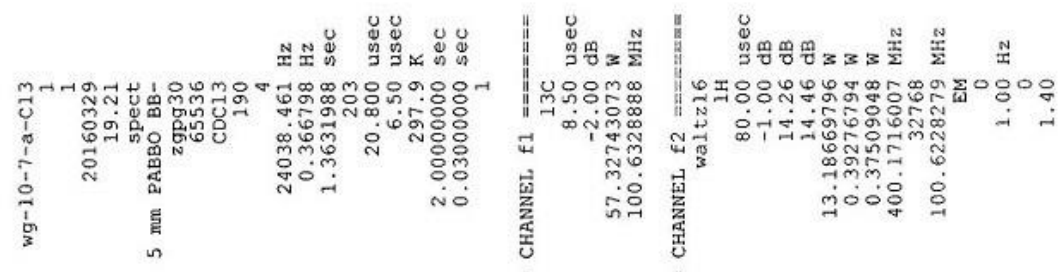

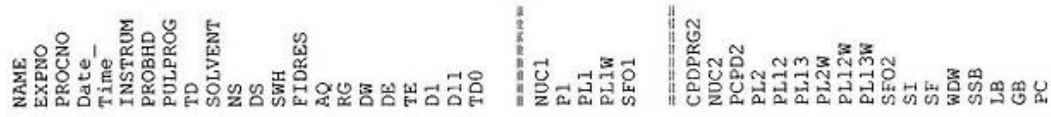

$\varepsilon L \cdot L>$

$09^{\circ} 7 \varepsilon$

$8 \varepsilon^{\circ} 0 t$

$\checkmark Z^{\prime} Z \boldsymbol{~}$

$S L \angle D-$

20.89

69.92

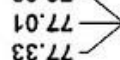

$80^{\circ} \nabla Z \downarrow$

ZS'७CL

cL.9Z1

$9 Z \cdot L Z$

81.821

t9.6Zl

98.เฉน

$७$ SEL

$6 \varepsilon$ เレレ

$89^{\circ} t 01$

$0 S^{\circ} \varepsilon L\llcorner-$

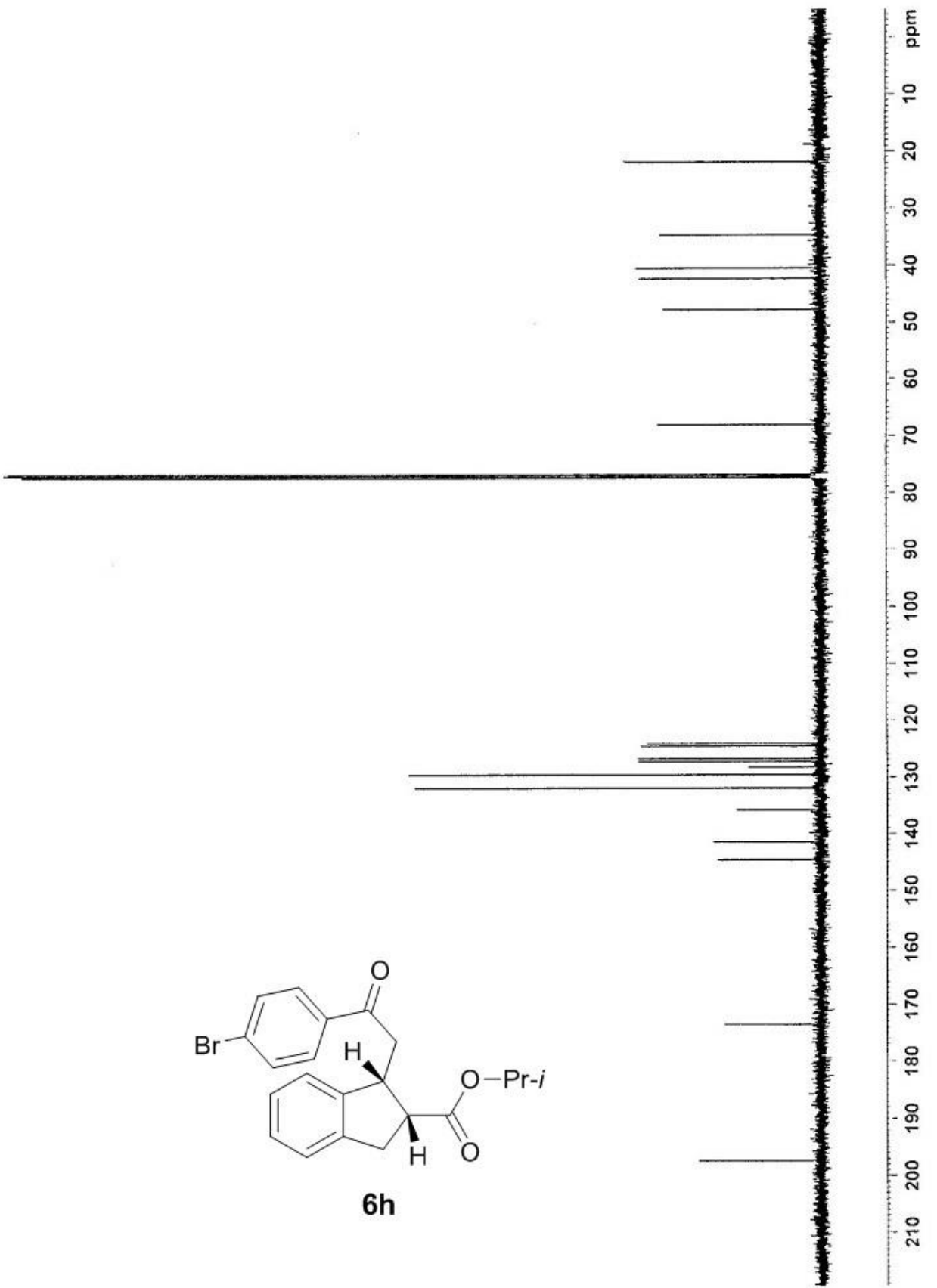



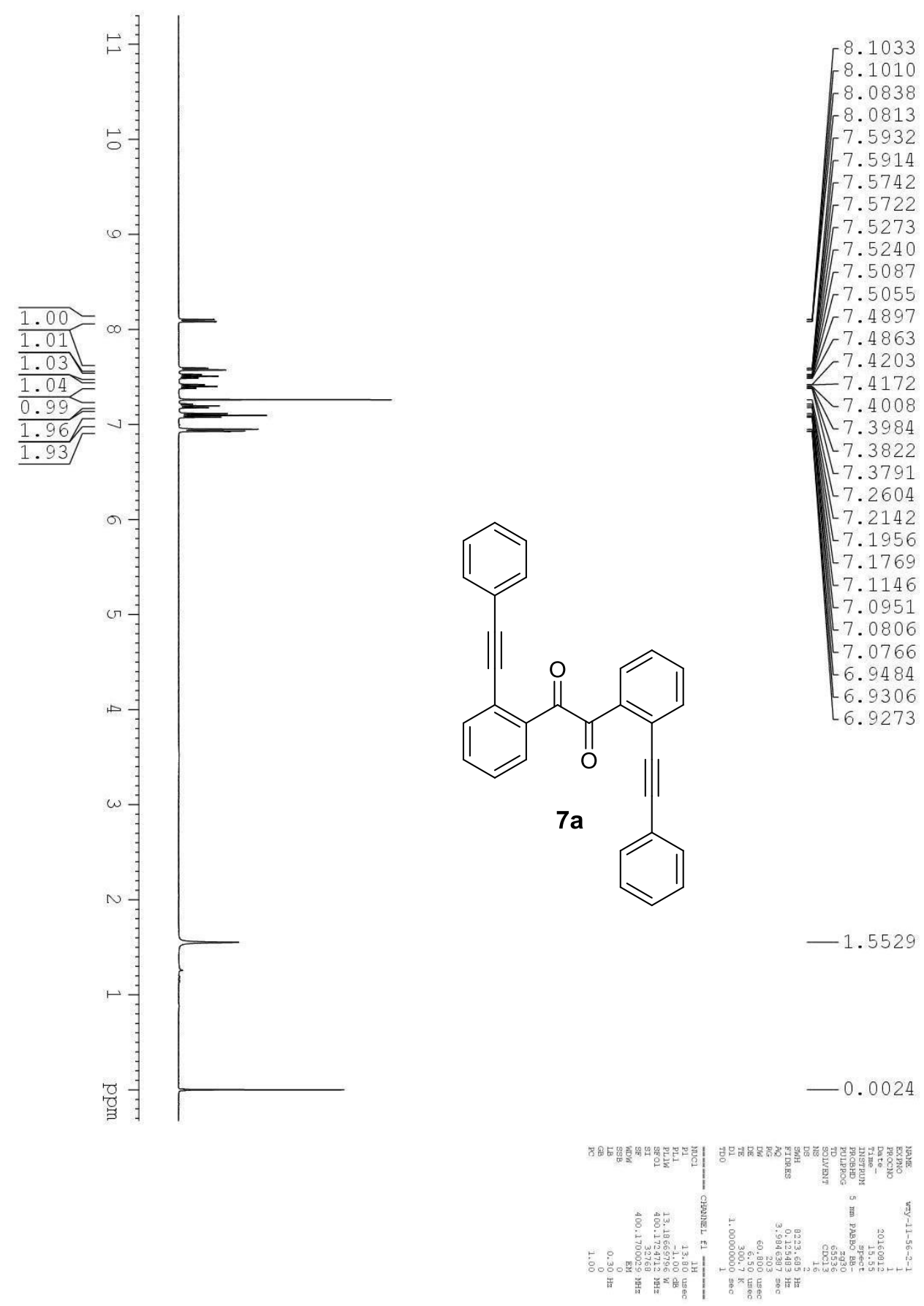


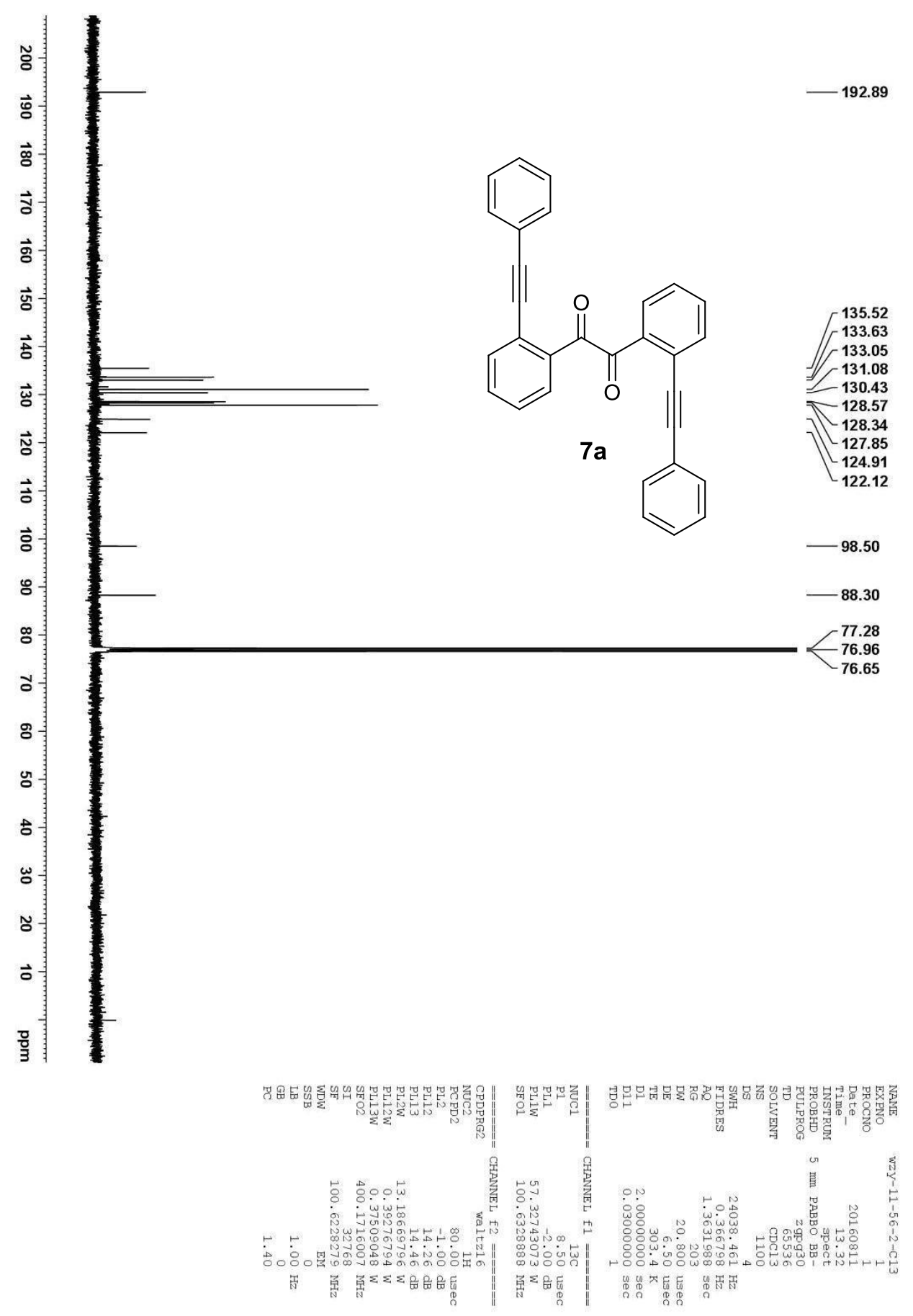




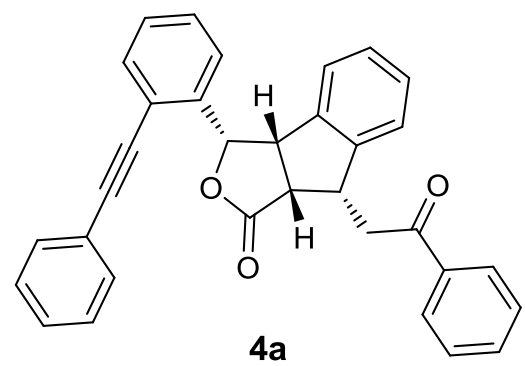

HPLC date of compound 4a: AD-H column, $90: 10$ hexane : IPA, flow rate: $1.0 \mathrm{ml} / \mathrm{min}, 254 \mathrm{~nm}, 25^{\circ} \mathrm{C}, 99 \%$ ee.

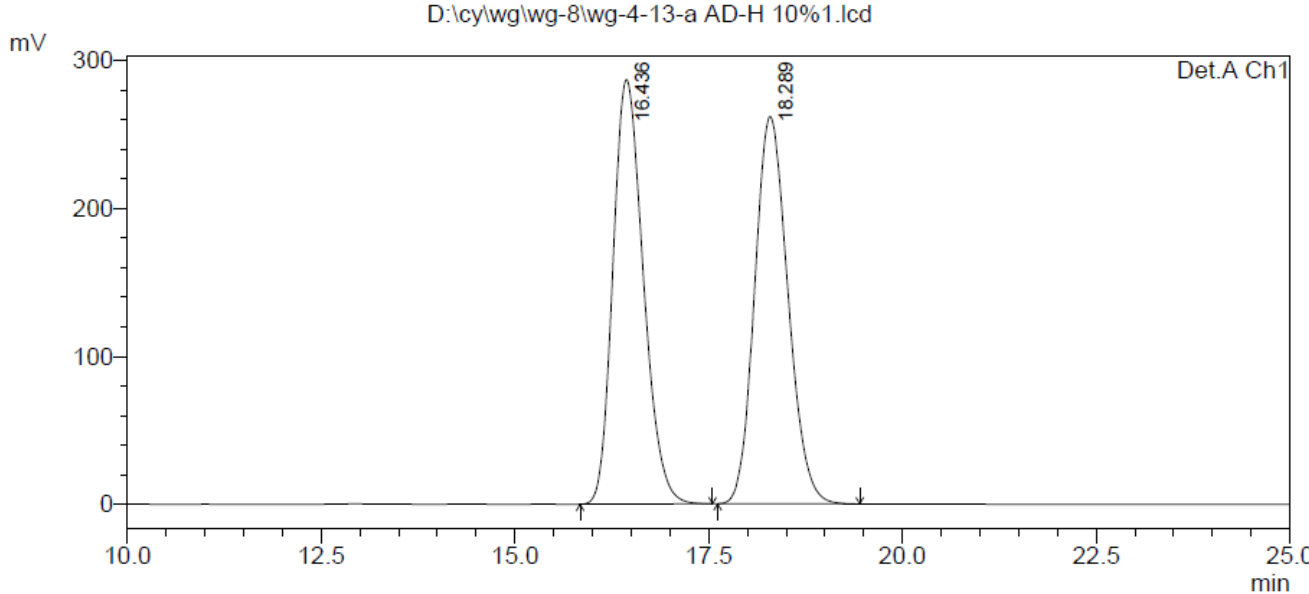

1 Det.A Ch1/254nm

Detector A Ch1 $254 \mathrm{~nm}$
\begin{tabular}{|r|r|r|r|r|r|}
\hline \multicolumn{1}{|c|}{ Peak\# } & Ret. Time & Area & Height & Area $\%$ & \multicolumn{1}{c|}{ Height $\%$} \\
\hline 1 & 16.436 & 7825378 & 287102 & 49.984 & 52.305 \\
\hline 2 & 18.289 & 7830293 & 261803 & 50.016 & 47.695 \\
\hline Total & & 15655671 & 548905 & 100.000 & 100.000 \\
\hline
\end{tabular}

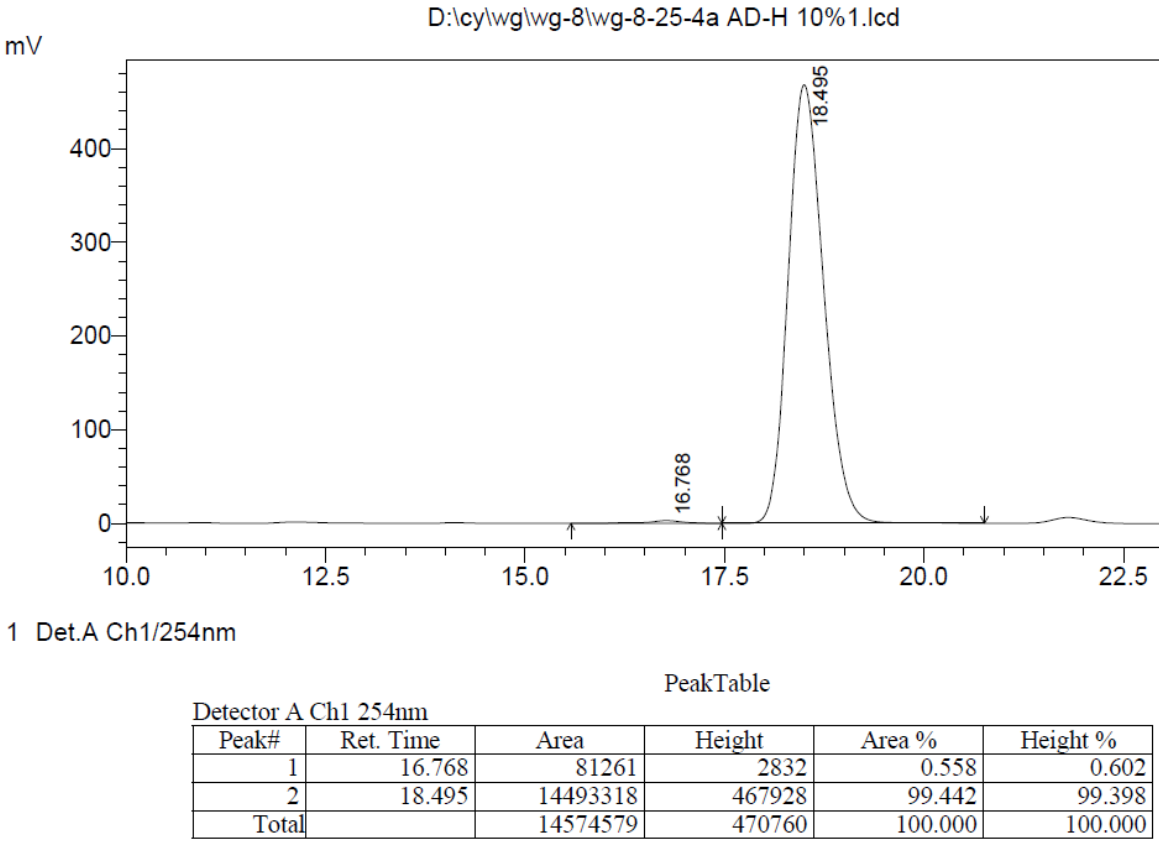




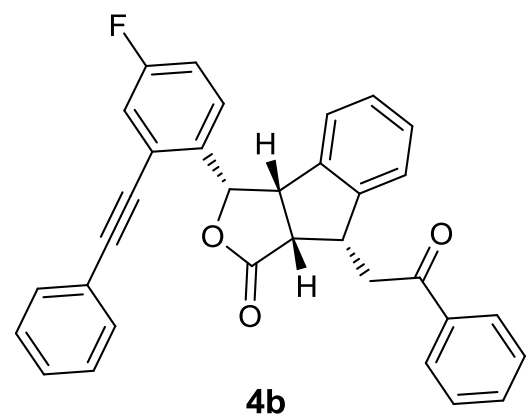

HPLC date of compound 4b: OD-H column, $80: 20$ hexane : IPA, flow rate: $1.0 \mathrm{ml} / \mathrm{min}, 254 \mathrm{~nm}, 25^{\circ} \mathrm{C}, 98 \%$ ee.

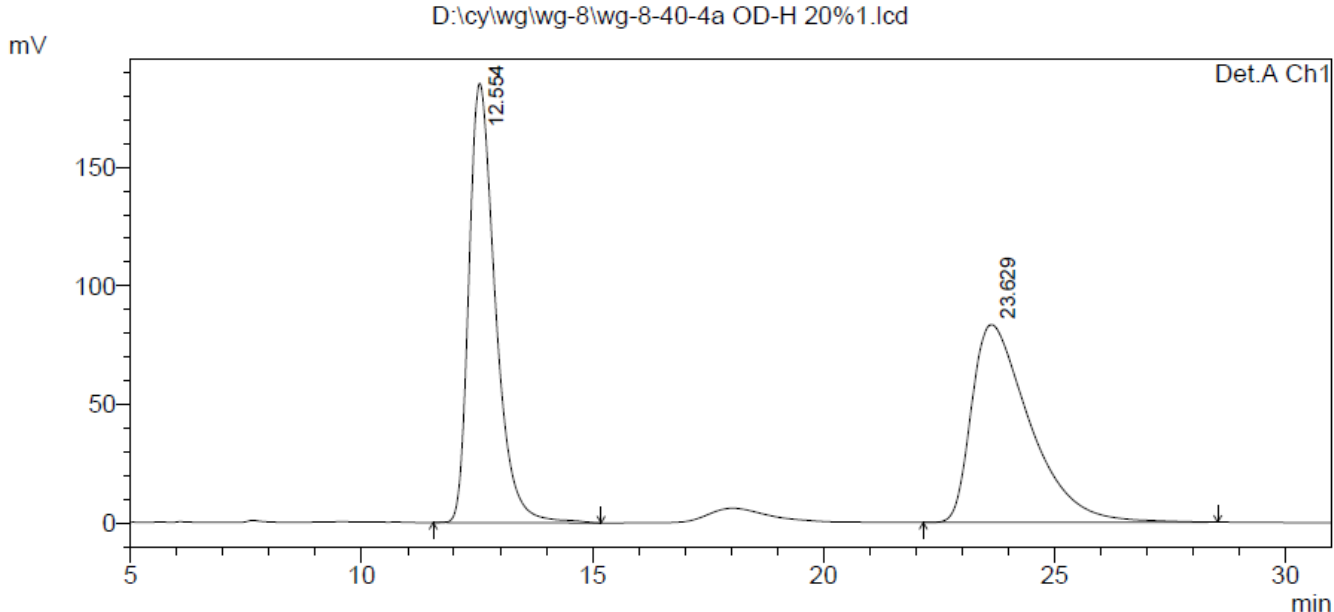

1 Det.A Ch1/254nm

Detector A Ch1 254nm
\begin{tabular}{|r|r|r|r|r|r|}
\hline \multicolumn{1}{|c|}{ Peak\# } & Ret. Time & \multicolumn{1}{|c|}{ Area } & Height & Area \% & \multicolumn{1}{|c|}{ Height \% } \\
\hline 1 & 12.554 & 7380456 & 185303 & 50.182 & 68.952 \\
\hline 2 & 23.629 & 7326971 & 83439 & 49.818 & 31.048 \\
\hline Total & & 14707427 & 268742 & 100.000 & 100.000 \\
\hline
\end{tabular}

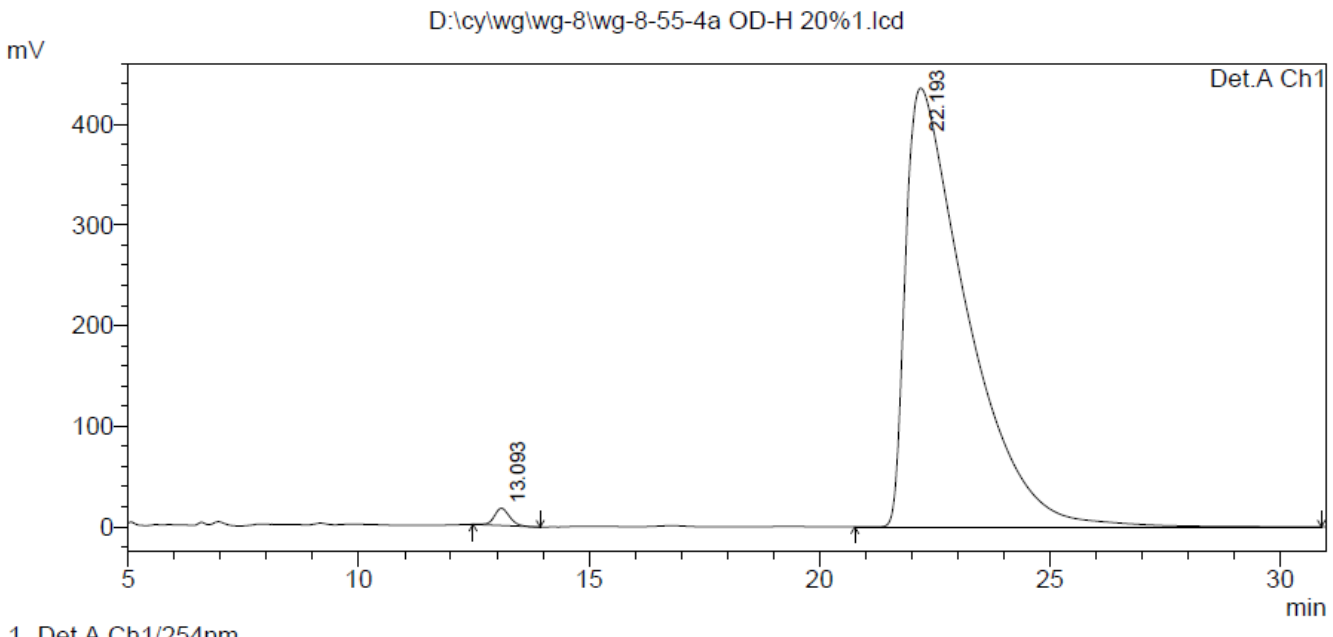

1 Det.A Ch1/254nm

PeakTable

Detector A Ch1 $254 \mathrm{~nm}$
\begin{tabular}{|r|r|r|r|r|r|}
\hline Peak\# & Ret. Time & Area & Height & Area $\%$ & \multicolumn{1}{c|}{ Height \% } \\
\hline 1 & 13.093 & 394983 & 17124 & 0.971 & 3.781 \\
\hline 2 & 22.193 & 40295466 & 435818 & 99.029 & 96.219 \\
\hline Total & & 40690450 & 452942 & 100.000 & 100.000 \\
\hline
\end{tabular}




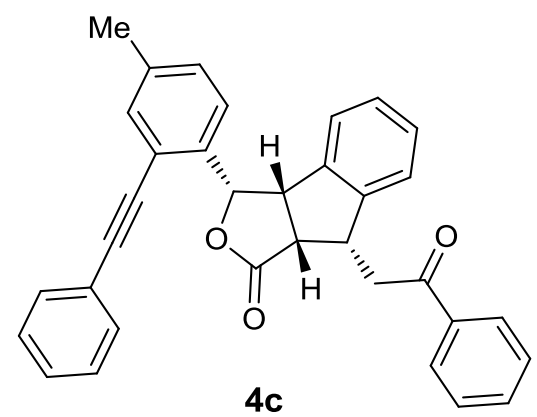

HPLC date of compound 4c: OD-H column, 80:20 hexane : IPA, flow rate: $1.0 \mathrm{ml} / \mathrm{min}, 254 \mathrm{~nm}, 25^{\circ} \mathrm{C},>99 \%$ ee.
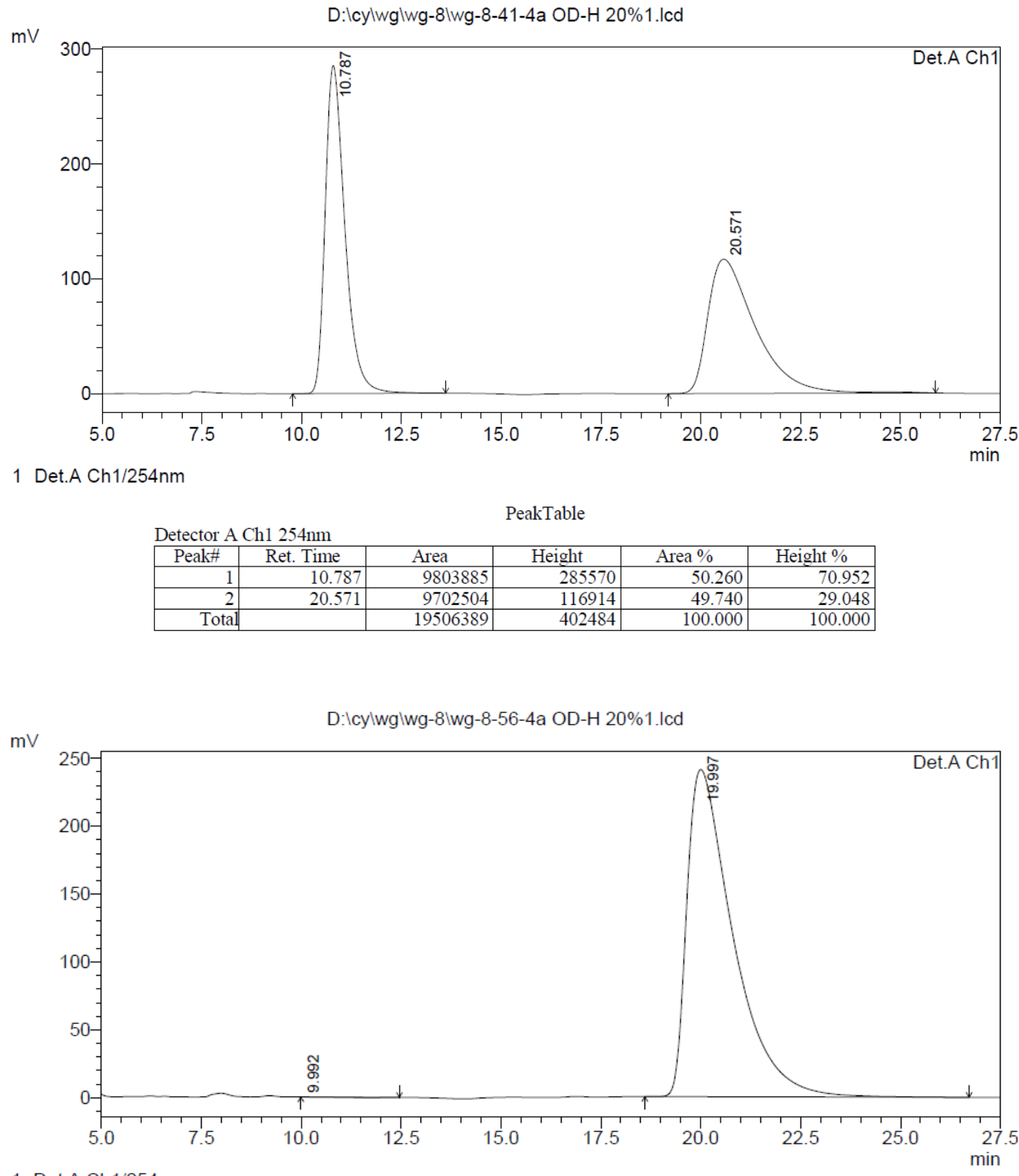

1 Det.A Ch1/254nm

Detector A Ch1 254nm
\begin{tabular}{|r|r|r|r|r|r|}
\hline \multicolumn{1}{|c|}{ Peak\# } & Ret. Time & \multicolumn{1}{|c|}{ Area } & Height & \multicolumn{1}{|c|}{ Area $\%$} & \multicolumn{1}{c|}{ Height $\%$} \\
\hline 1 & 9.992 & -18791 & 0 & -0.097 & 0.000 \\
\hline 2 & 19.997 & 19364177 & 241120 & 100.097 & 100.000 \\
\hline Total & & 19345386 & 241120 & 100.000 & 100.000 \\
\hline
\end{tabular}




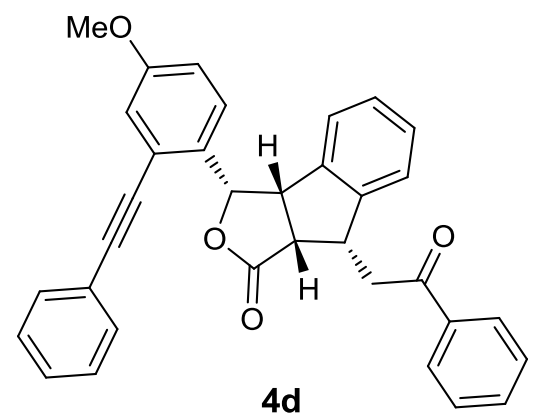

HPLC date of compound 4d: OD-H column, 80:20 hexane : IPA, flow rate: $1.0 \mathrm{ml} / \mathrm{min}, 254 \mathrm{~nm}, 25^{\circ} \mathrm{C}, 96 \%$ ee.

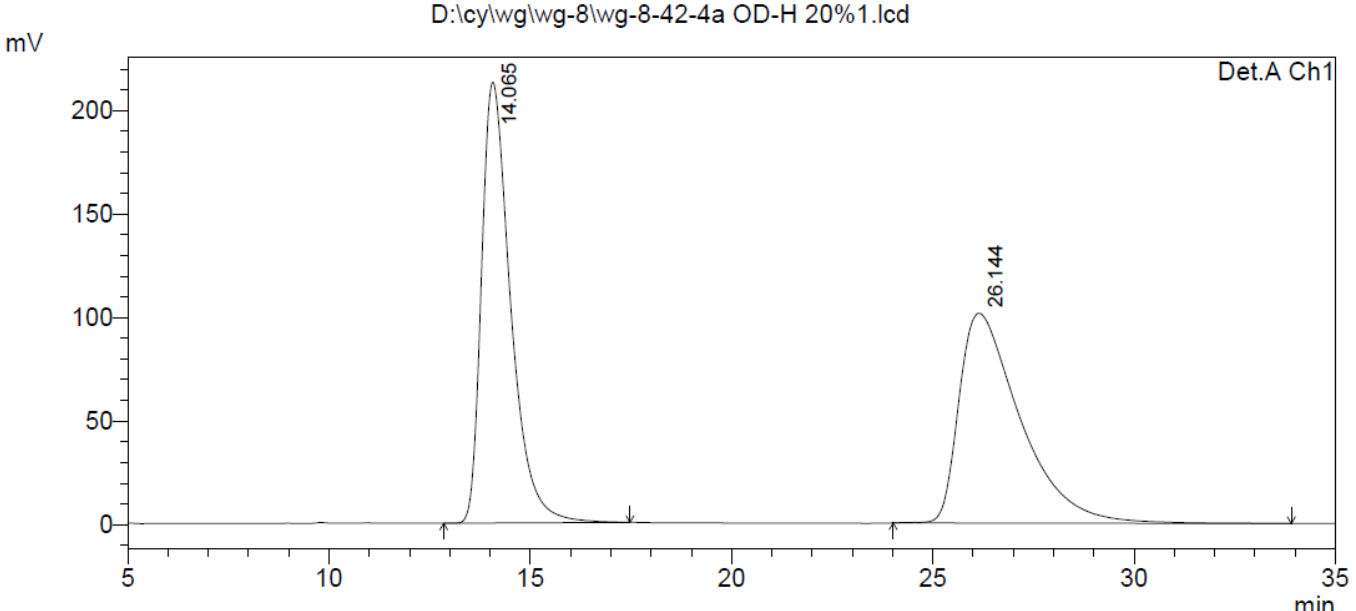

1 Det.A Ch1/254nm

Detector A Ch1 $254 \mathrm{~nm}$
\begin{tabular}{|r|r|r|r|r|r|}
\hline Peak\# & Ret. Time & Area & Height & Area $\%$ & Height $\%$ \\
\hline 1 & 14.065 & 10706158 & 212881 & 49.927 & 67.748 \\
\hline 2 & 26.144 & 10737518 & 101343 & 50.073 & 32.252 \\
\hline Total & & 21443676 & 314223 & 100.000 & 100.000 \\
\hline
\end{tabular}

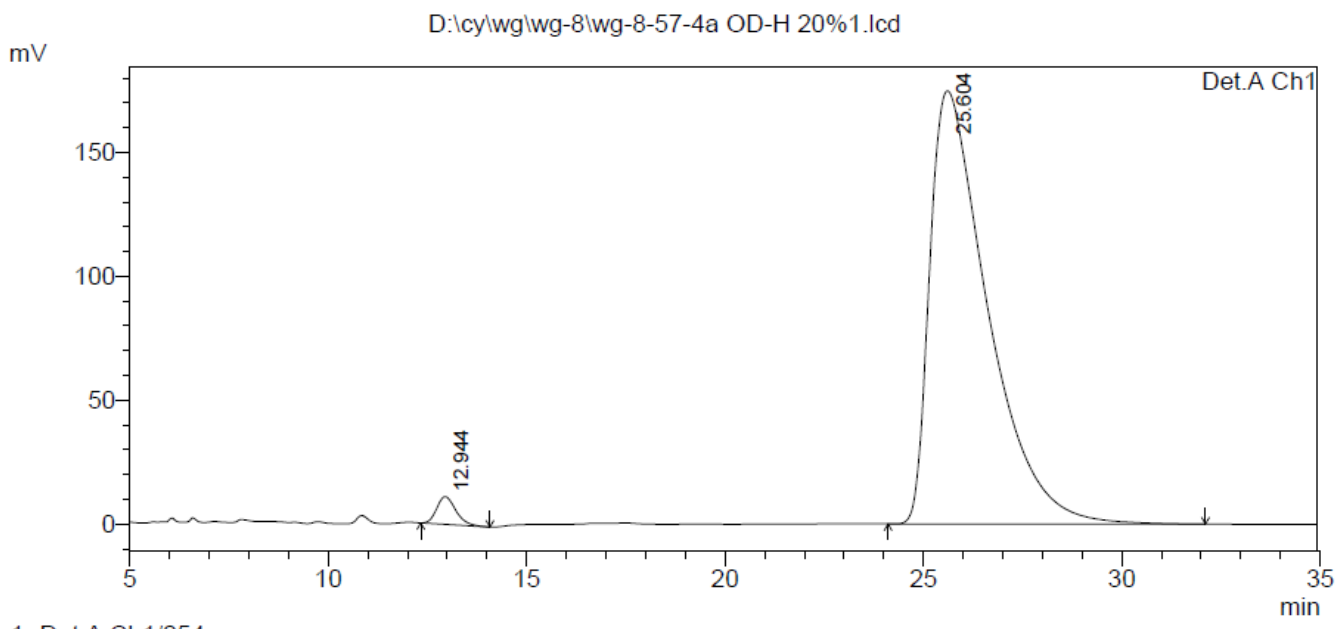

1 Det A Ch1/254nm

Detector A Ch1 254nm
\begin{tabular}{|r|r|r|r|r|r|}
\hline Peak\# & Ret. Time & Area & Height & Area $\%$ & \multicolumn{1}{c|}{ Height $\%$} \\
\hline 1 & 12.944 & 374168 & 11182 & 2.067 & \multicolumn{1}{c|}{6.016} \\
\hline 2 & 25.604 & 17725973 & 174693 & 97.933 & 93.984 \\
\hline Total & & 18100141 & 185876 & 100.000 & 100.000 \\
\hline
\end{tabular}




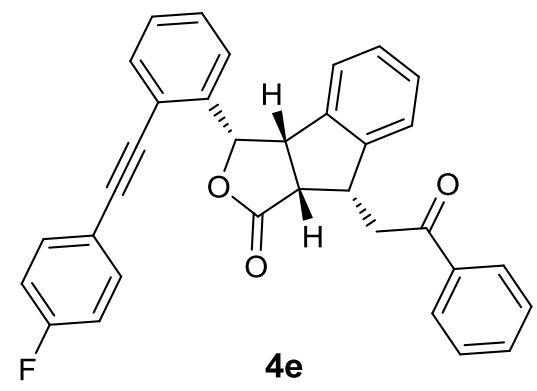

HPLC date of compound 4e: OD-H column, 80:20 hexane : IPA, flow rate: $1.0 \mathrm{ml} / \mathrm{min}, 254 \mathrm{~nm}, 25^{\circ} \mathrm{C},>99 \%$ ee.

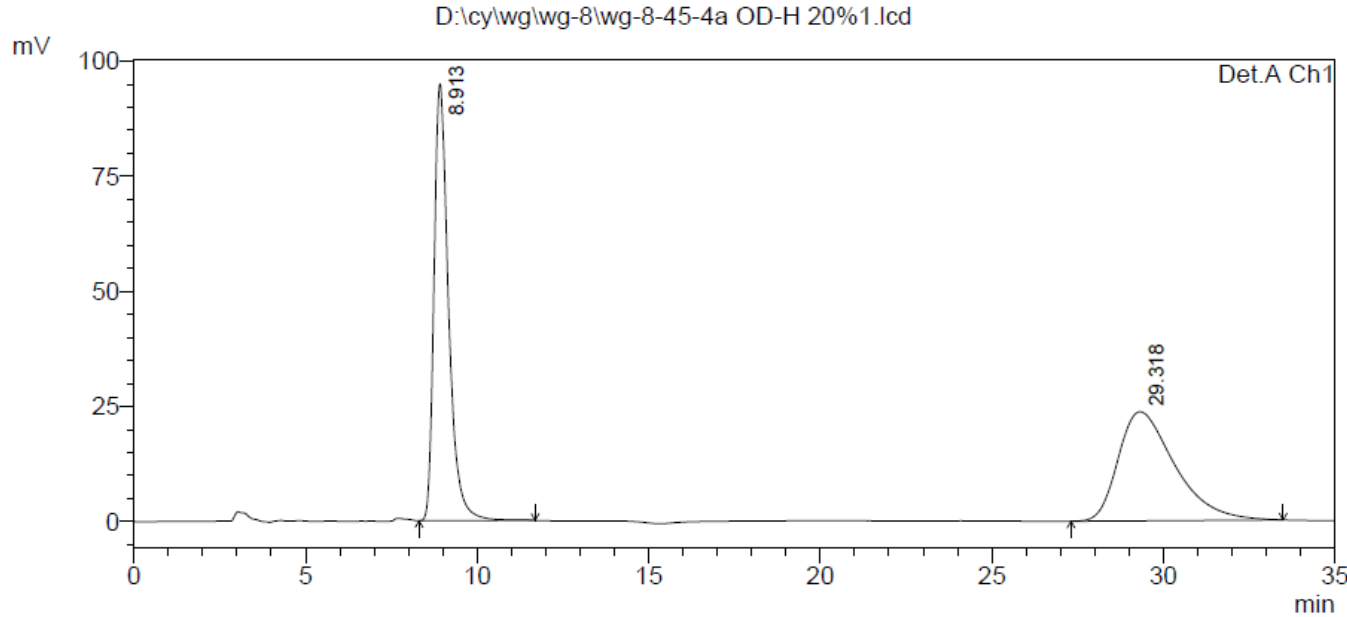

1 Det.A Ch1/254nm

PeakTable

Detector A Ch1 254nm
\begin{tabular}{|r|r|r|r|r|r|}
\hline \multicolumn{1}{|c|}{ Peak\# } & Ret. Time & \multicolumn{1}{c|}{ Area } & \multicolumn{1}{c|}{ Height } & Area \% & Height $\%$ \\
\hline 1 & 8.913 & 2796775 & 94874 & 50.742 & 80.017 \\
\hline 2 & 29.318 & 2714970 & 23693 & 49.258 & 19.983 \\
\hline Total & & 5511745 & 118568 & 100.000 & 100.000 \\
\hline
\end{tabular}

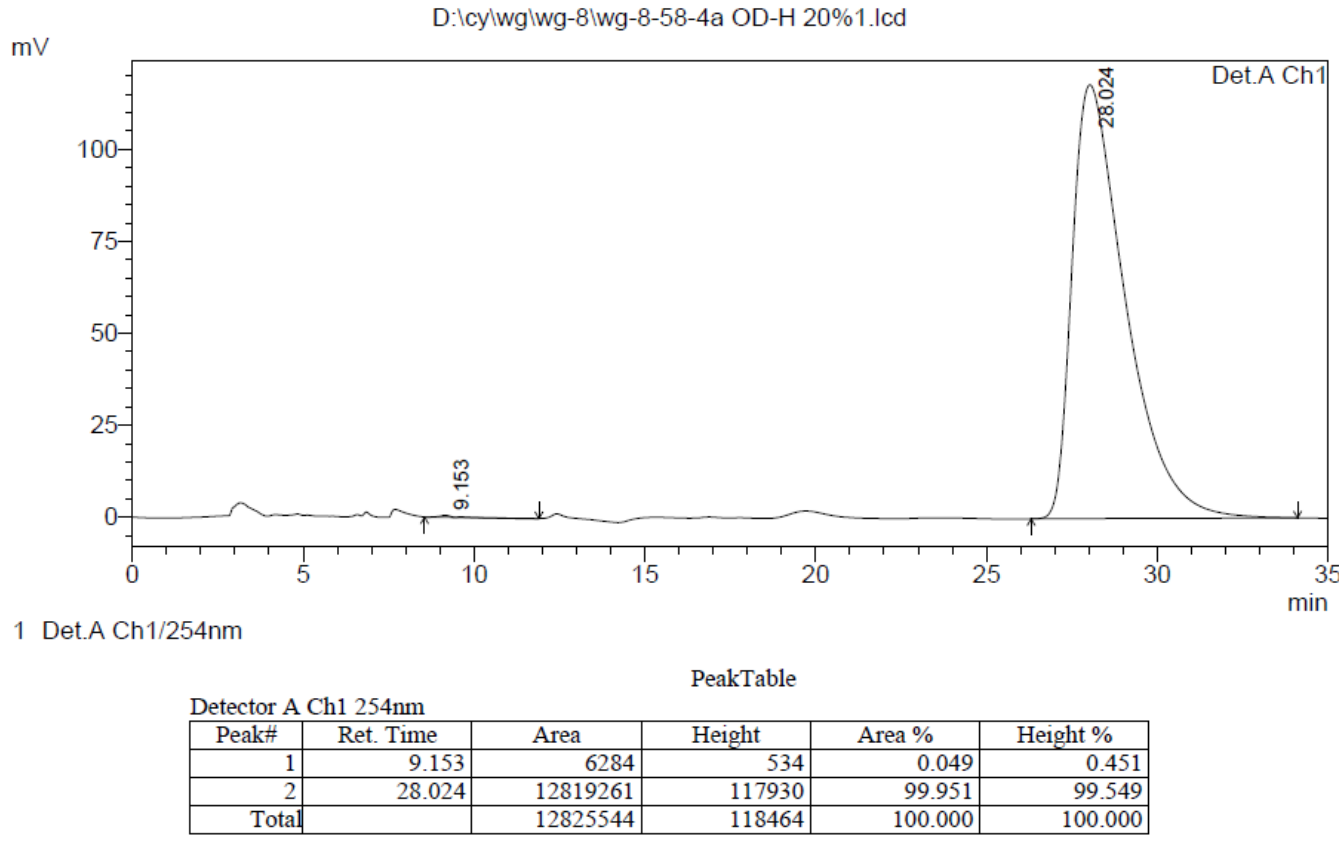




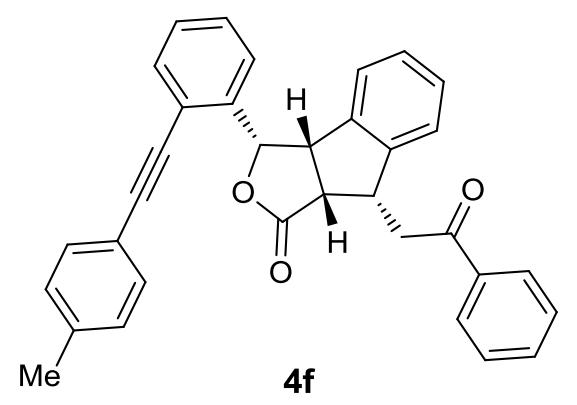

HPLC date of compound 4f: OD-H column, 80:20 hexane : IPA, flow rate: $1.0 \mathrm{ml} / \mathrm{min}, 254 \mathrm{~nm}, 25^{\circ} \mathrm{C}, 99 \%$ ee.

$\mathrm{mV}$

D:lcylwglwg-8lwg-8-46-4a OD-H 20\%1.lcd

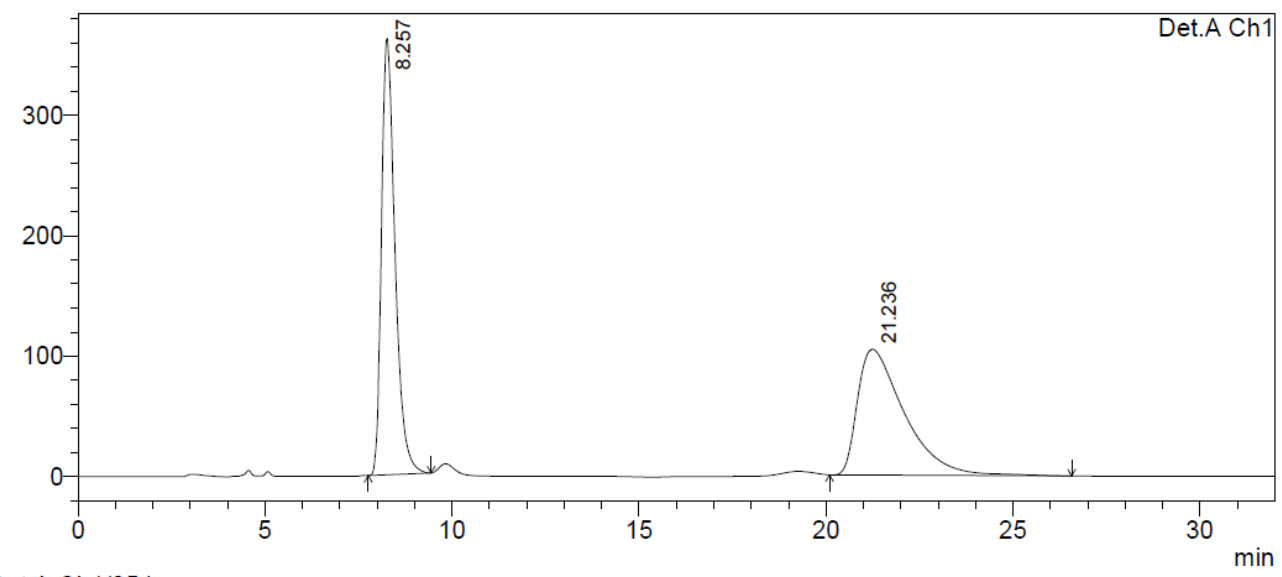

1 Det.A Ch1/254nm

PeakTable

Detector A Ch1 254nm
\begin{tabular}{|r|r|r|r|r|r|}
\hline Peak\# & Ret. Time & \multicolumn{1}{c|}{ Area } & Height & Area $\%$ & Height $\%$ \\
\hline 1 & 8.257 & 9146190 & 362267 & 50.138 & 77.615 \\
\hline 2 & 21.236 & 9095954 & 104484 & 49.862 & 22.385 \\
\hline Total & & 18242144 & 466752 & 100.000 & 100.000 \\
\hline
\end{tabular}

$\mathrm{mV}$

D:lcylwglwg-8lwg-8-59-4a OD-H 20\%1.lcd

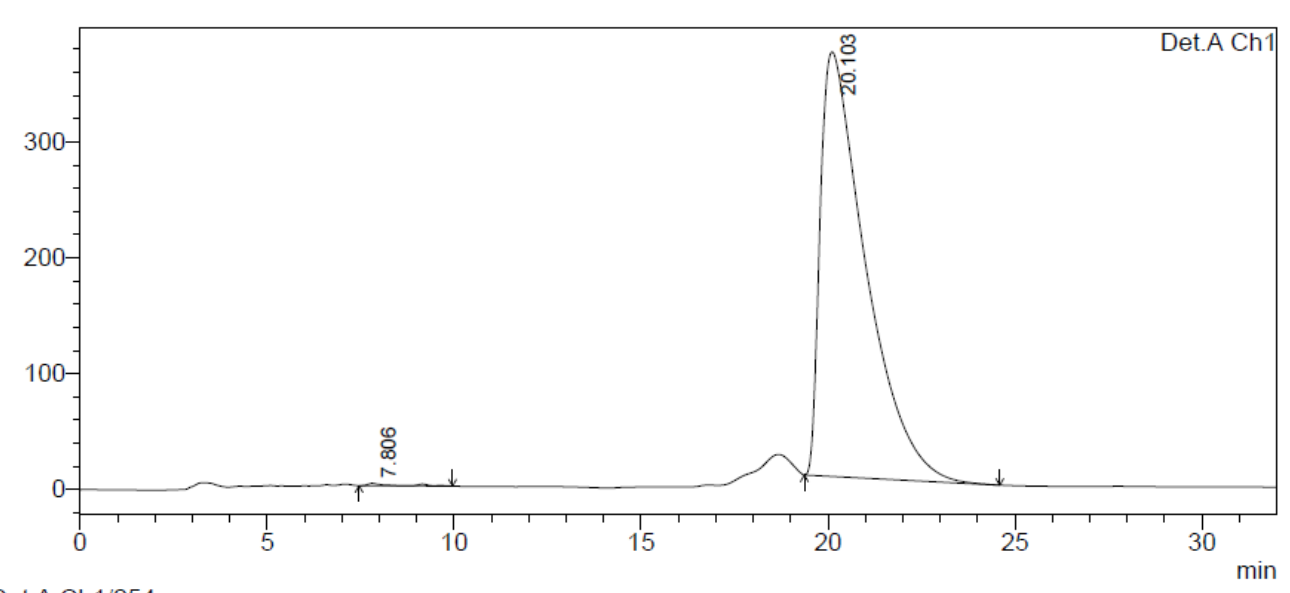

1 Det.A Ch1/254nm

PeakTable

Detector A Ch1 $254 \mathrm{~nm}$
\begin{tabular}{|r|r|r|r|r|r|}
\hline Peak\# & Ret. Time & Area & Height & Area $\%$ & \multicolumn{1}{|c|}{ Height \% } \\
\hline 1 & 7.806 & 96221 & 2152 & 0.310 & 0.584 \\
\hline 2 & 20.103 & 30988067 & 366525 & 99.690 & 99.416 \\
\hline Total & & 31084287 & 368677 & 100.000 & 100.000 \\
\hline
\end{tabular}




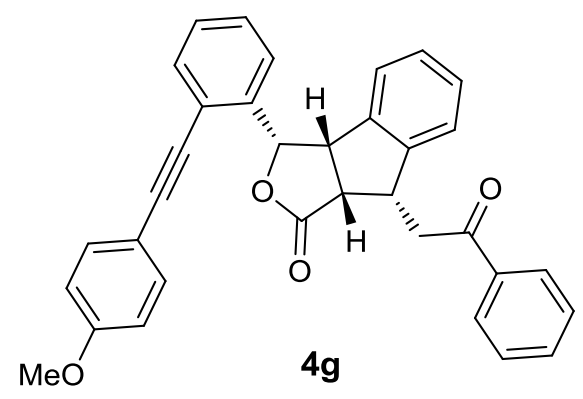

HPLC date of compound 4g: OD-H column, 80:20 hexane : IPA, flow rate: $1.0 \mathrm{ml} / \mathrm{min}, 254 \mathrm{~nm}, 25^{\circ} \mathrm{C}, 98 \%$ ee.

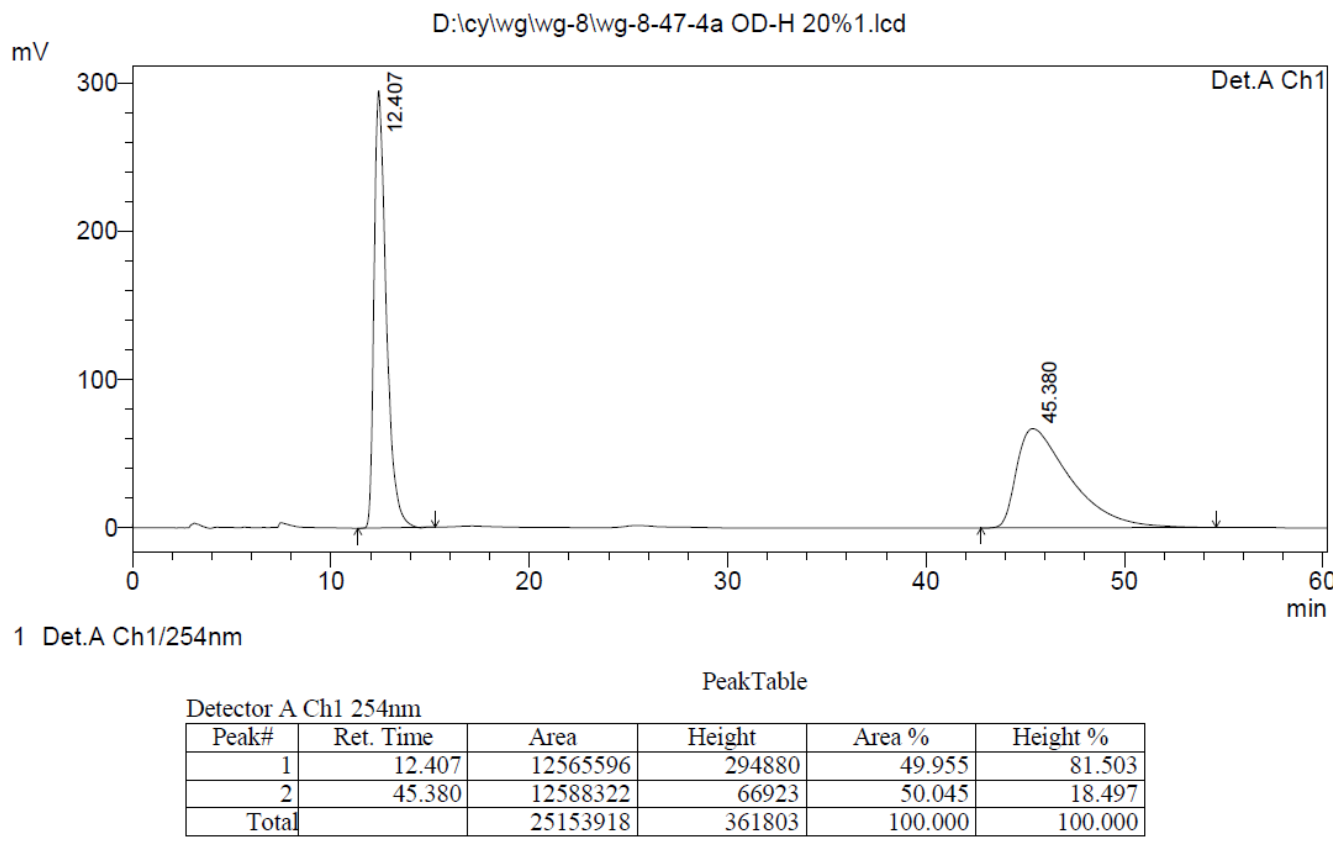

$\mathrm{mV}$

D:lcylwglwg-8lwg-8-60-4a OD-H 20\%1.Icd

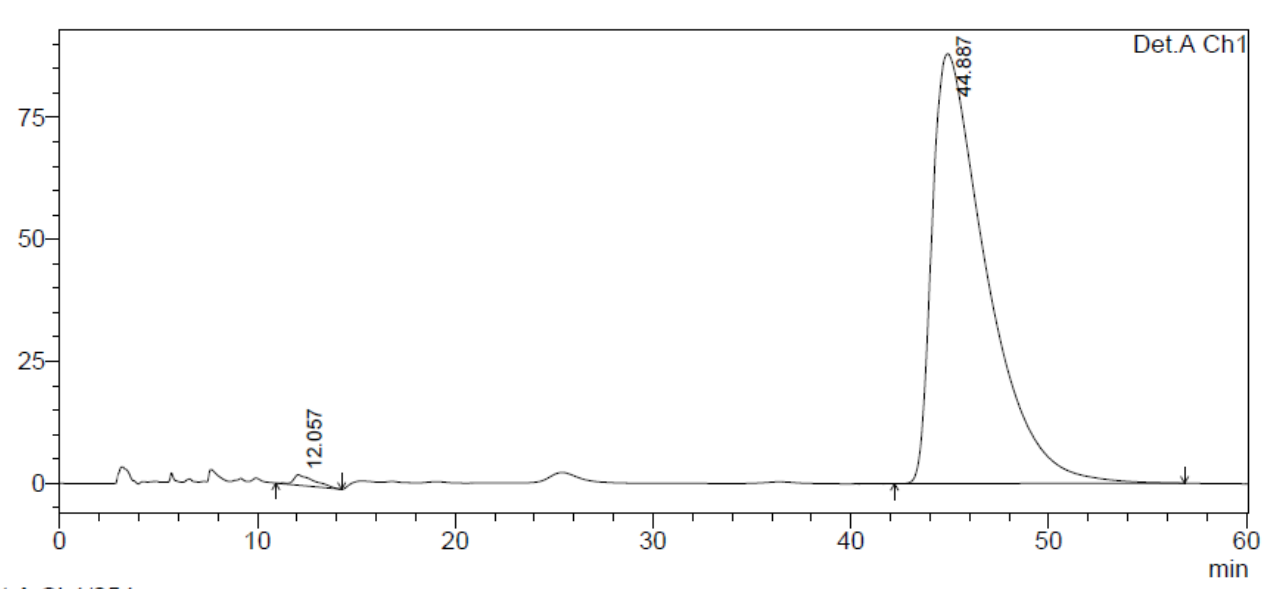

1 Det.A Ch1/254nm

Detector A Ch1 $254 \mathrm{~nm}$
\begin{tabular}{|r|r|r|r|r|r|}
\hline Peak\# & Ret. Time & \multicolumn{1}{|c|}{ Area } & \multicolumn{1}{|c|}{ Height } & Area $\%$ & Height $\%$ \\
\hline 1 & 12.057 & 161145 & 2126 & 0.947 & 2.357 \\
\hline 2 & 44.887 & 16849955 & 88099 & 99.053 & 97.643 \\
\hline Total & & 17011100 & 90225 & 100.000 & 100.000 \\
\hline
\end{tabular}




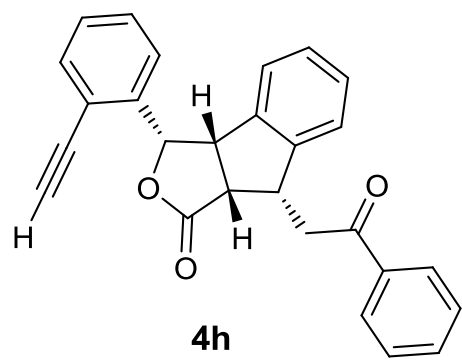

HPLC date of compound $4 \mathbf{h}$ : AD-H column, 90:10 hexane : IPA, flow rate: $1.0 \mathrm{ml} / \mathrm{min}, 254 \mathrm{~nm}, 25^{\circ} \mathrm{C},>99 \%$ ee.

$\mathrm{mV}$

D:Icylwglwg-8lwg-9-19-4a AD-H 10\%1.lcd

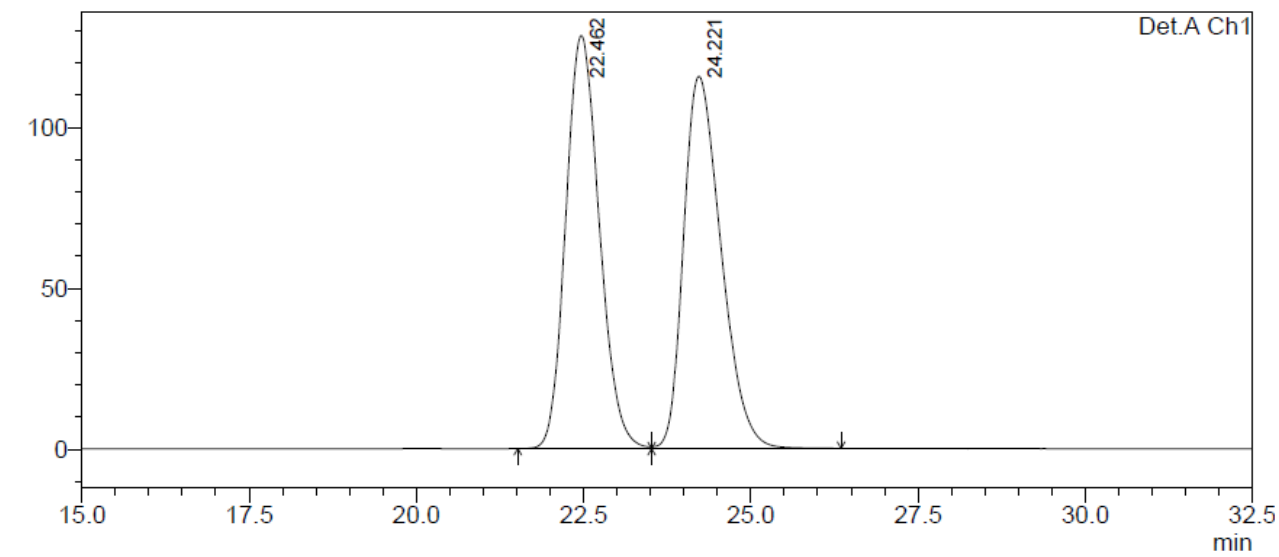

1 Det.A Ch1/254nm

PeakTable
Detector A Ch1 254nm
\begin{tabular}{|r|r|r|r|r|r|}
\hline Peak\# & Ret. Time & Area & Height & \multicolumn{1}{c|}{ Area \% } & \multicolumn{1}{c|}{ Height \% } \\
\hline 1 & 22.462 & 4478743 & 128156 & 49.894 & 52.600 \\
\hline 2 & 24.221 & 4497706 & 115486 & 50.106 & 47.400 \\
\hline Total & & 8976450 & 243643 & 100.000 & 100.000 \\
\hline
\end{tabular}

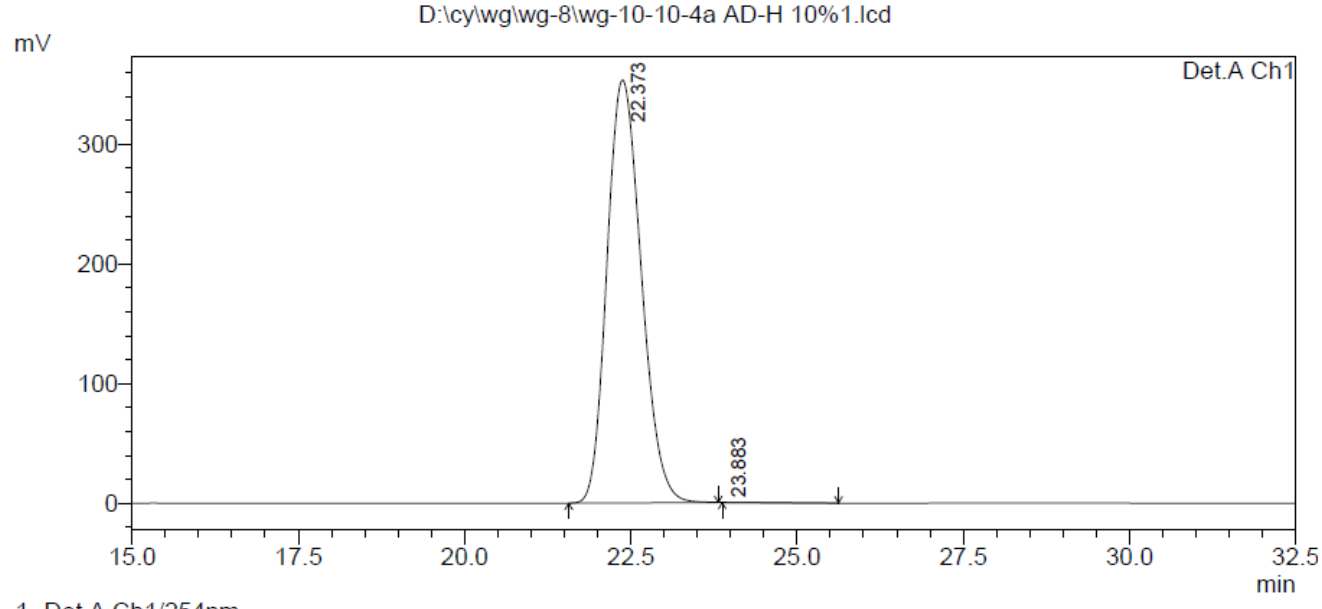

1 Det.A Ch1/254nm

DeakTable
\begin{tabular}{|r|r|r|r|r|r|}
\hline Peak\# A Ch1 254nm & Ret. Time & \multicolumn{1}{c|}{ Area } & Height & \multicolumn{1}{c|}{ Area \% } & \multicolumn{1}{c|}{ Height $\%$} \\
\hline 1 & 22.373 & 12663266 & 353312 & 100.047 & 100.002 \\
\hline 2 & 23.883 & -5998 & -7 & -0.047 & -0.002 \\
\hline Total & & 12657268 & 353305 & 100.000 & 100.000 \\
\hline
\end{tabular}




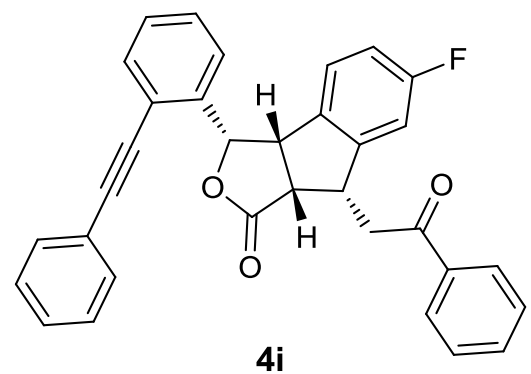

HPLC date of compound 4i: AD-H column, 80:20 hexane : IPA, flow rate: $1.0 \mathrm{ml} / \mathrm{min}, 254 \mathrm{~nm}, 25^{\circ} \mathrm{C},>99 \%$ ee.

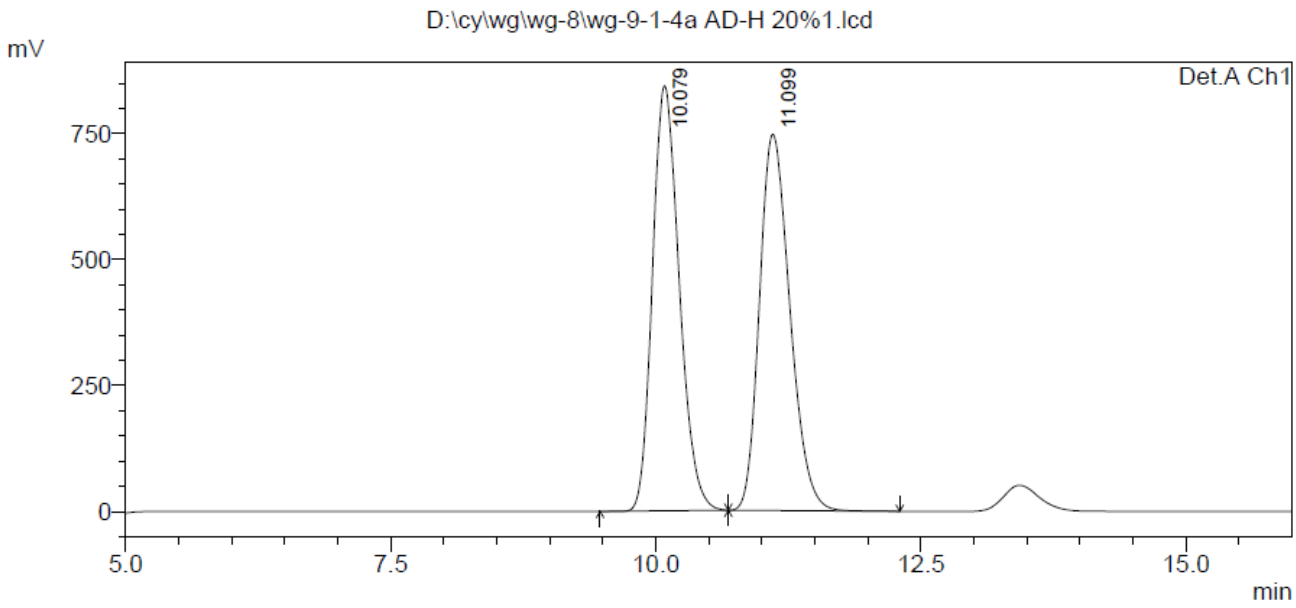

1 Det.A Ch $1 / 254 \mathrm{~nm}$

\begin{tabular}{|c|c|c|c|c|c|}
\hline \multicolumn{6}{|c|}{ Pea } \\
\hline Peak\# & Ret Time & & & & Hoilto \\
\hline 1 & 10.079 & 14840371 & 843960 & 49893 & 53.042 \\
\hline 2 & 11.099 & 14903998 & 747169 & 50.107 & 46.958 \\
\hline Tota & & 29744369 & 1591130 & 100.000 & 100.000 \\
\hline
\end{tabular}

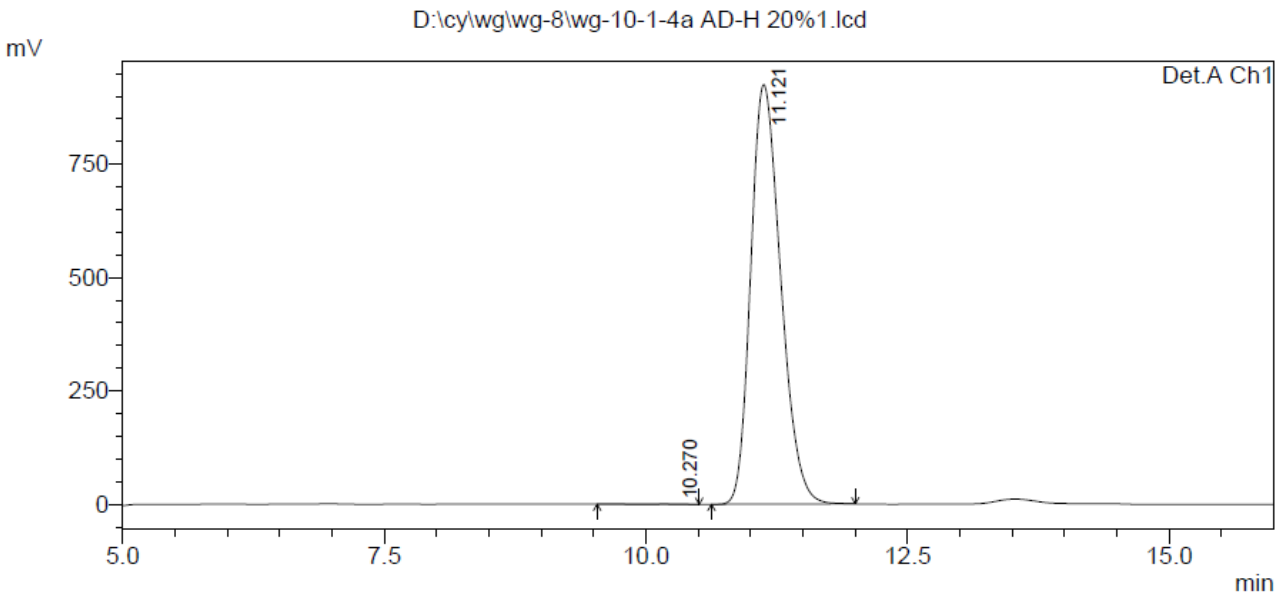

1 Det.A Ch $1 / 254 \mathrm{~nm}$

Detector A Ch1 $254 \mathrm{~nm}$
\begin{tabular}{|r|r|r|r|r|r|}
\hline Peak\# & Ret. Time & \multicolumn{1}{c|}{ Area } & \multicolumn{1}{c|}{ Height } & Area $\%$ & Height $\%$ \\
\hline 1 & 10.270 & 7336 & 454 & 0.040 & 0.049 \\
\hline 2 & 11.121 & 18449011 & 925558 & 99.960 & 99.951 \\
\hline Total & & 18456347 & 926012 & 100.000 & 100.000 \\
\hline
\end{tabular}




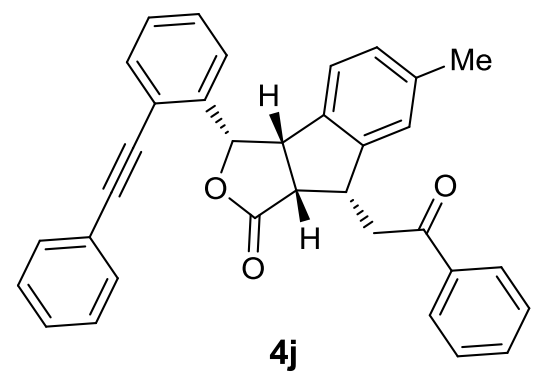

HPLC date of compound 4j: OD-H column, $80: 20$ hexane : IPA, flow rate: $1.0 \mathrm{ml} / \mathrm{min}, 254 \mathrm{~nm}, 25^{\circ} \mathrm{C},>99 \%$ ee.

$\mathrm{mV}$

D:lcylwglwg-8Iwg-9-2-4a OD-H 20\%1.Icd

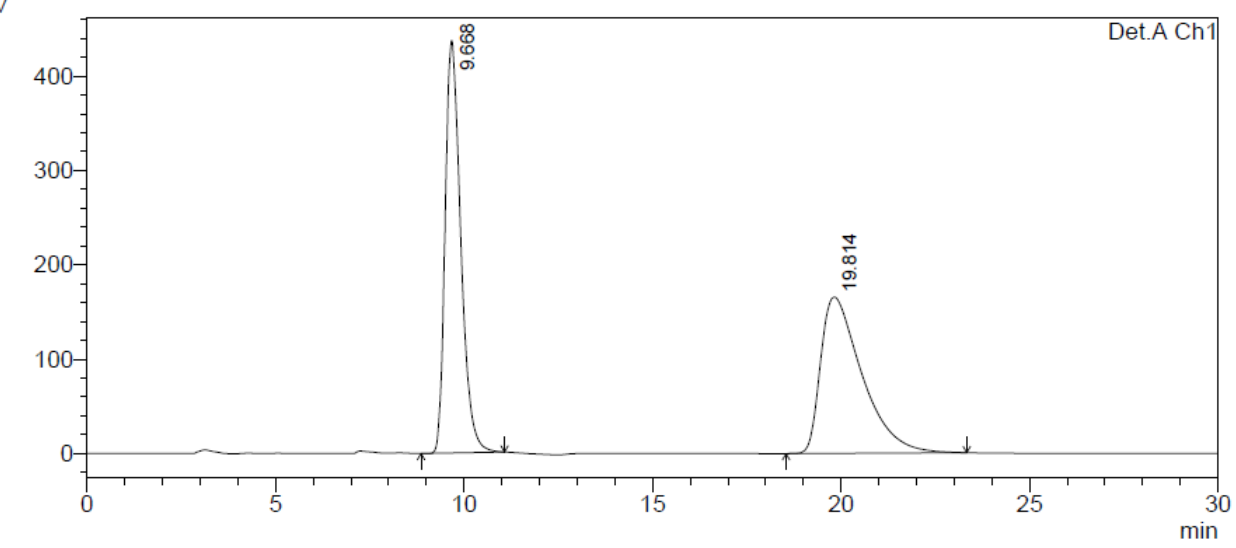

1 Det.A Ch1/254nm

Detector A Ch1 $254 \mathrm{~nm}$
\begin{tabular}{|r|r|r|r|r|r|}
\hline \multicolumn{1}{|c|}{ Peak\# } & Ret. Time & \multicolumn{1}{c|}{ Area } & Height & Area $\%$ & Height $\%$ \\
\hline 1 & 9.668 & 12618198 & 437133 & 50.004 & 72.505 \\
\hline 2 & 19.814 & 12616177 & 165768 & 49.996 & 27.495 \\
\hline Total & & 25234374 & 602900 & 100.000 & 100.000 \\
\hline
\end{tabular}

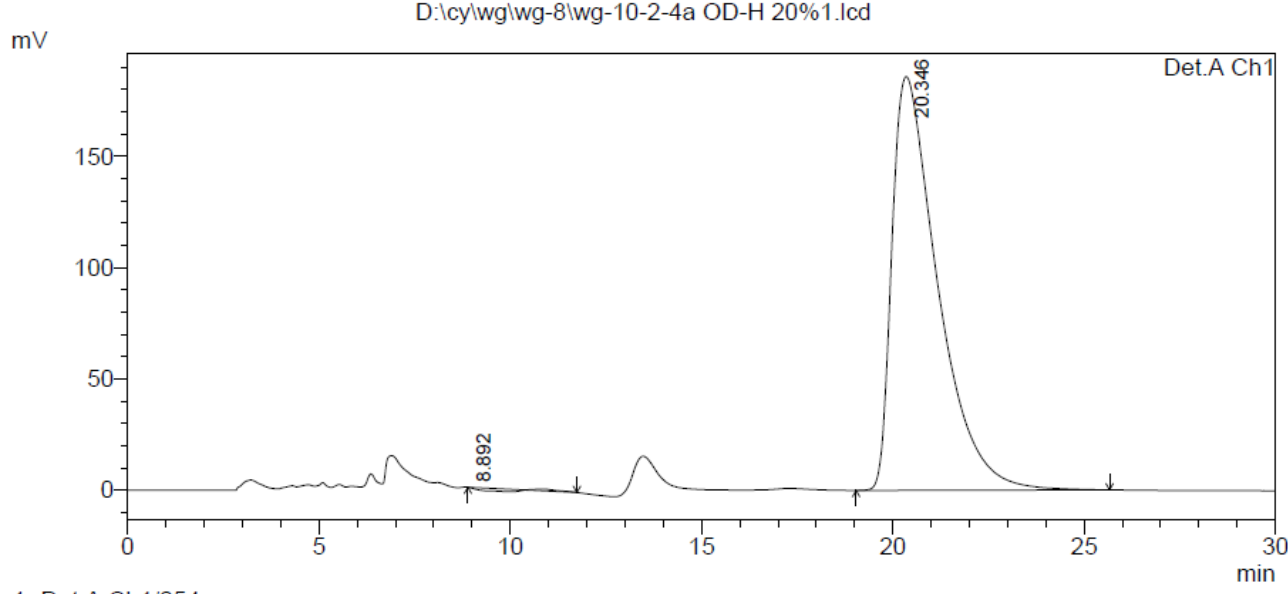

1 Det.A Ch1/254nm

PeakTable

\begin{tabular}{|c|c|c|c|c|c|}
\hline \multirow{2}{*}{\multicolumn{6}{|c|}{ PeakTable }} \\
\hline & & & & & \\
\hline Peak\# & Ret. Time & Area & Height & Area \% & Height \% \\
\hline 1 & 8.892 & -48013 & 0 & -0.312 & 0.000 \\
\hline 2 & 20.346 & 15427117 & 185848 & 100.312 & 100.000 \\
\hline Total & & 15379104 & 185848 & 100.000 & 100.000 \\
\hline
\end{tabular}




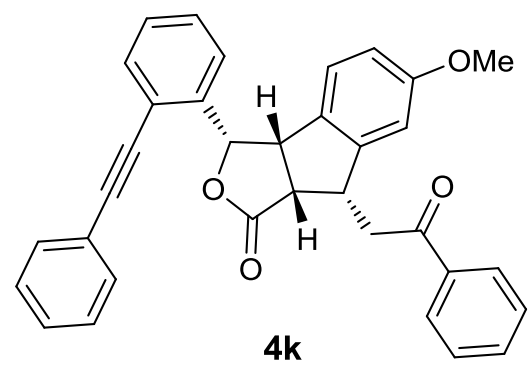

HPLC date of compound 4k: AD-H column, 80:20 hexane : IPA, flow rate: $1.0 \mathrm{ml} / \mathrm{min}, 254 \mathrm{~nm}, 25^{\circ} \mathrm{C},>99 \%$ ee.

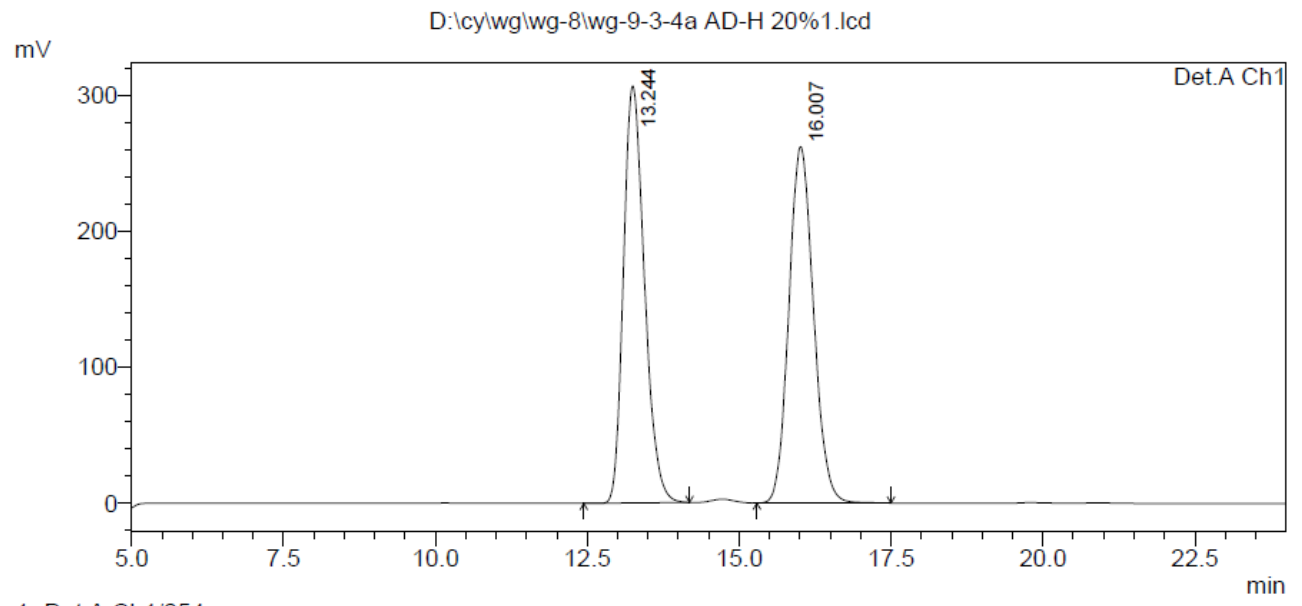

1 Det.A Ch $1 / 254 \mathrm{~nm}$

Detector A Ch1 $254 \mathrm{~nm}$
\begin{tabular}{|r|r|r|r|r|r|}
\hline Peak\# & Ret. Time & \multicolumn{1}{c|}{ Area } & Height & Area $\%$ & Height $\%$ \\
\hline 1 & 13.244 & 7368284 & 306748 & 49.882 & 53.923 \\
\hline 2 & 16.007 & 7403075 & 262110 & 50.118 & 46.077 \\
\hline Total & & 14771359 & 568858 & 100.000 & 100.000 \\
\hline
\end{tabular}

$\mathrm{mV}$

D: lcylwglwg-8lwg-10-3-4a AD-H 20\%1.Icd

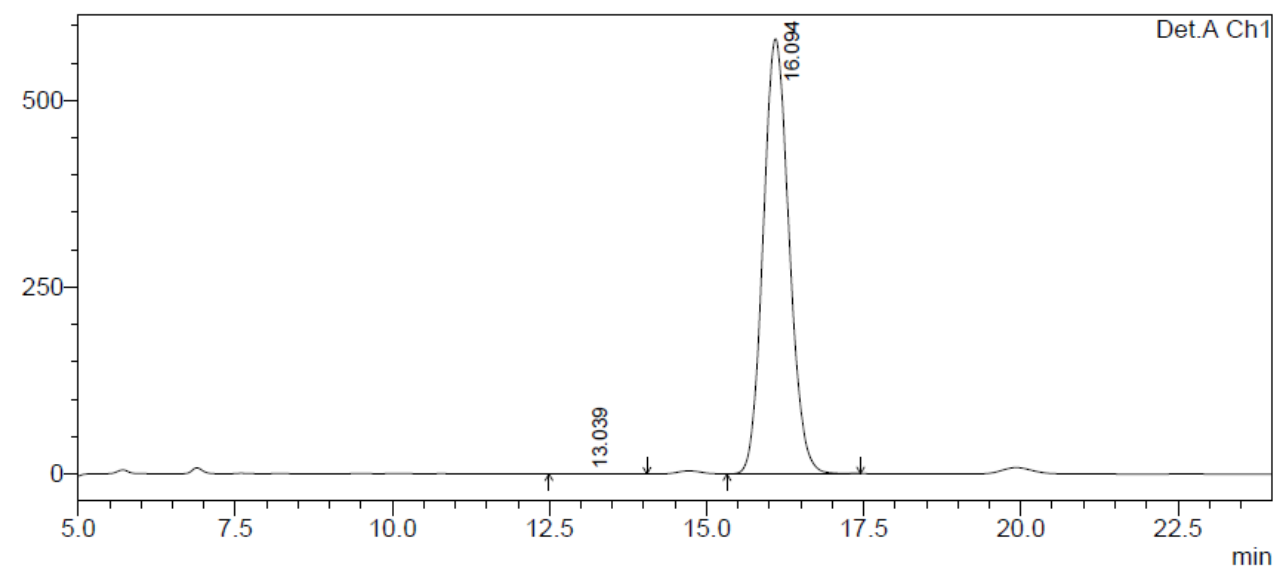

1 Det.A Ch1/254nm

PeakTable

Detector A Ch1 $254 \mathrm{~nm}$
\begin{tabular}{|r|r|r|r|r|r|}
\hline Peak\# & Ret. Time & \multicolumn{1}{c|}{ Area } & \multicolumn{1}{c|}{ Height } & Area \% & Height $\%$ \\
\hline 1 & 13.039 & 10837 & 262 & 0.065 & 0.045 \\
\hline 2 & 16.094 & 16687982 & 582267 & 99.935 & 99.955 \\
\hline Total & & 16698819 & 582529 & 100.000 & 100.000 \\
\hline
\end{tabular}




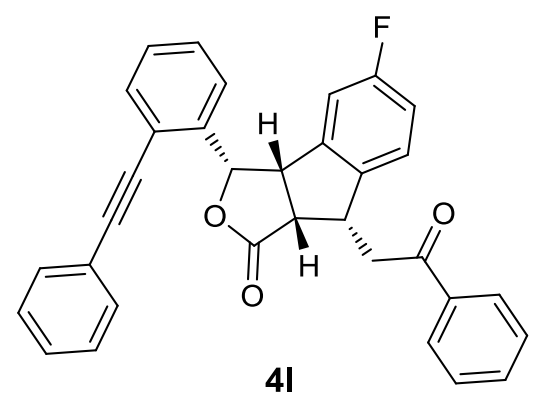

HPLC date of compound 4l: AD-H column, 80:20 hexane : IPA, flow rate: $1.0 \mathrm{ml} / \mathrm{min}, 254 \mathrm{~nm}, 25^{\circ} \mathrm{C},>99 \%$ ee.

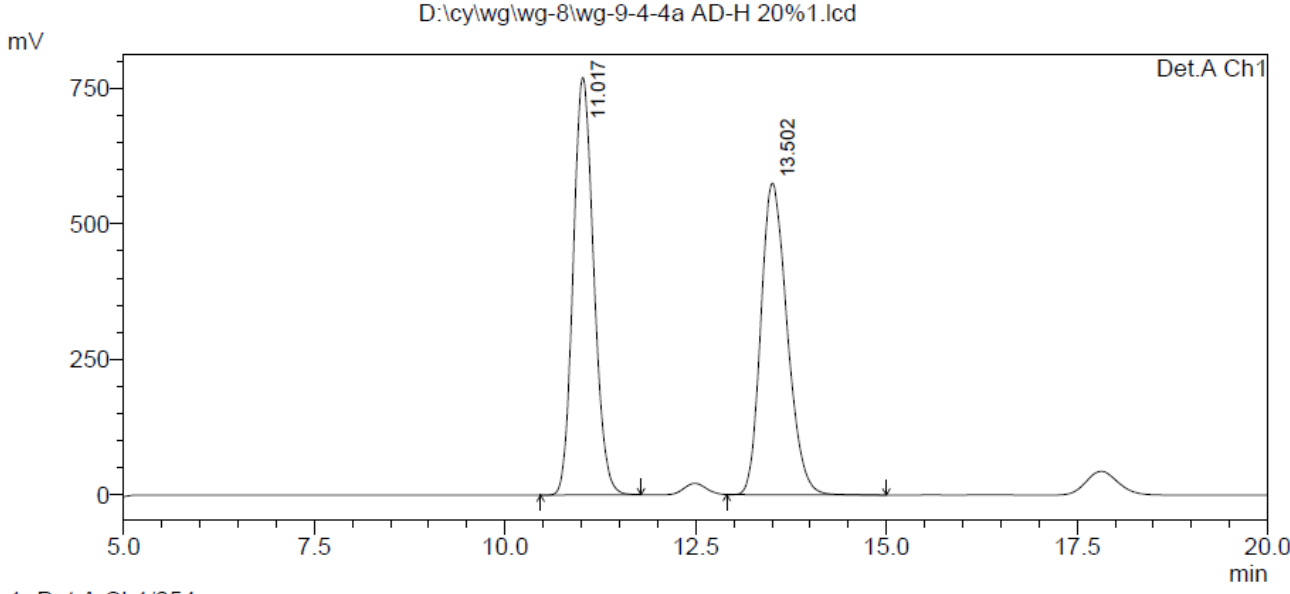

1 Det.A Ch $1 / 254 \mathrm{~nm}$

Detector A Ch1 $254 \mathrm{~nm}$
\begin{tabular}{|r|r|r|r|r|r|}
\hline Peak\# & Ret. Time & Area & \multicolumn{1}{c|}{ Height } & Area $\%$ & \multicolumn{1}{c|}{ Height $\%$} \\
\hline 1 & 11.017 & 14418939 & 769240 & 51.144 & 57.250 \\
\hline 2 & 13.502 & 13773793 & 574405 & 48.856 & 42.750 \\
\hline Total & & 28192732 & 1343645 & 100.000 & 100.000 \\
\hline
\end{tabular}

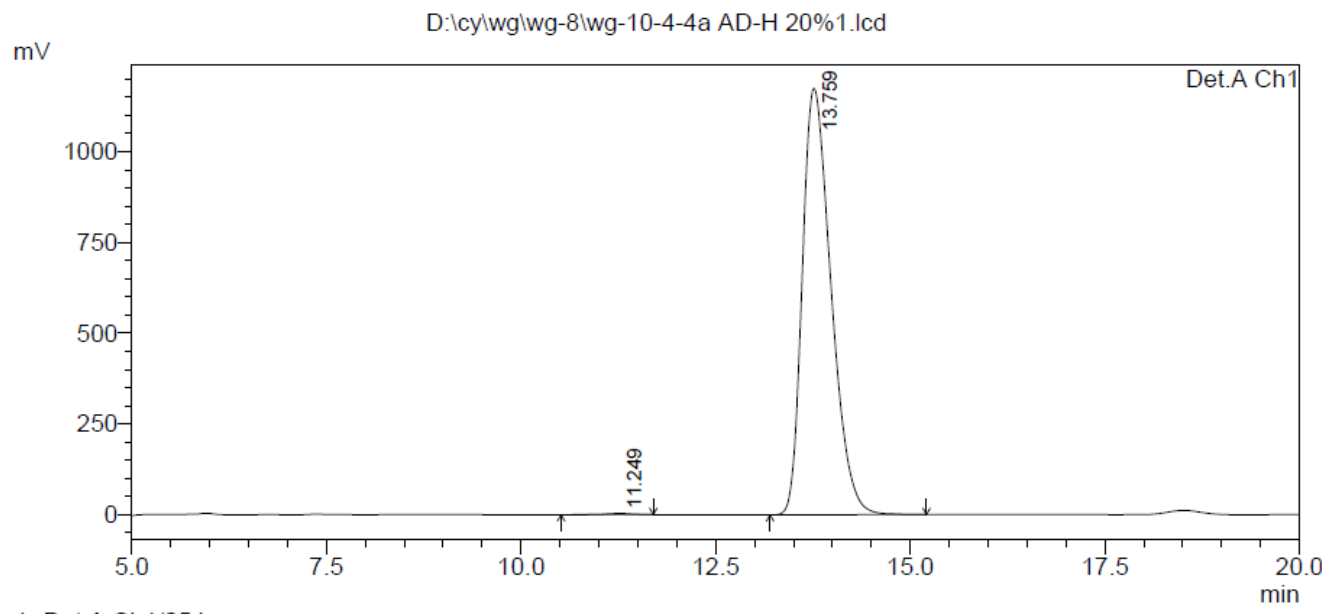

1 Det.A Ch $1 / 254 \mathrm{~nm}$

PeakTable
\begin{tabular}{|r|r|r|r|r|r|}
\hline \multicolumn{1}{|c|}{ Detector A Ch1 $254 \mathrm{~nm}$} \\
\hline Peak\# & Ret. Time & Area & Height & Area \% & Height $\%$ \\
\hline 1 & 11.249 & 69130 & 3238 & 0.229 & 0.275 \\
\hline 2 & 13.759 & 30170793 & 1174790 & 99.771 & 99.725 \\
\hline Total & & 30239923 & 1178028 & 100.000 & 100.000 \\
\hline
\end{tabular}




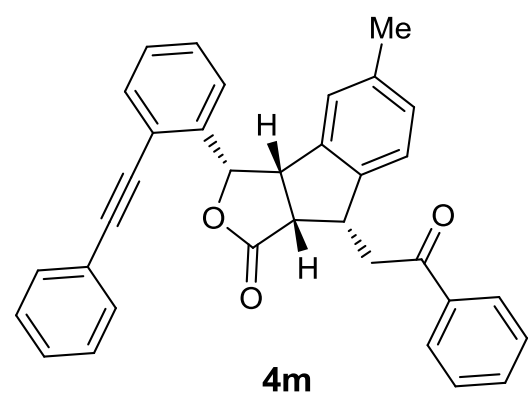

HPLC date of compound 4m: OD-H column, 80:20 hexane : IPA, flow rate: $1.0 \mathrm{ml} / \mathrm{min}, 254 \mathrm{~nm}, 25^{\circ} \mathrm{C}, 99 \%$ ee. $\mathrm{mV}$

D:Icylwglwg-8lwg-9-5-4a OD-H 20\%1.Icd

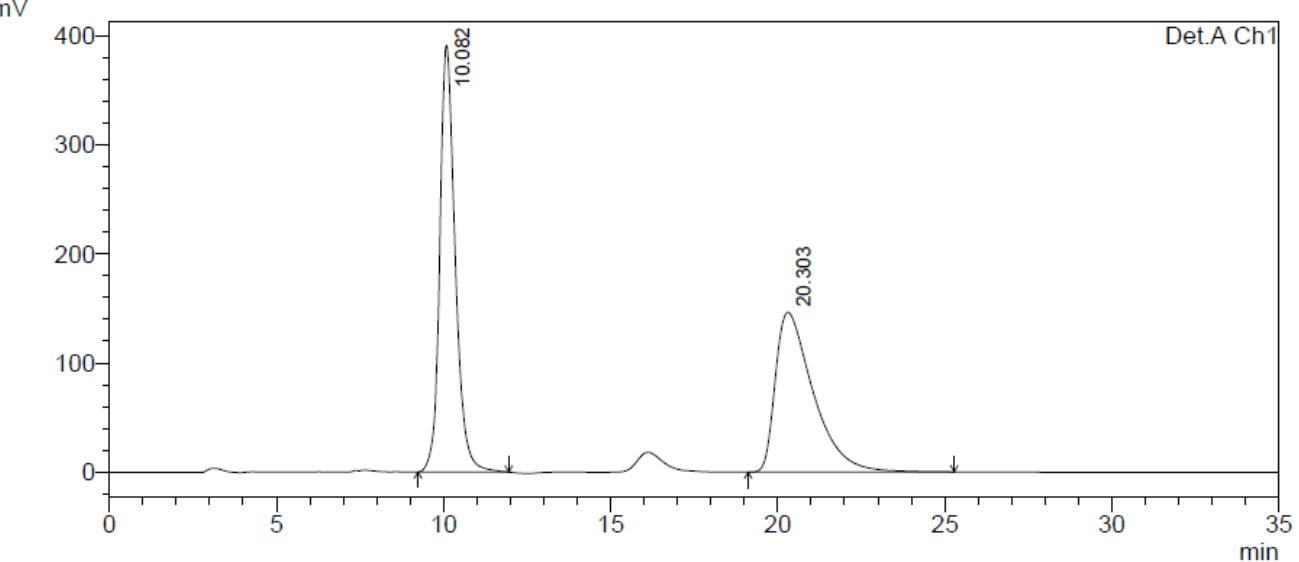

1 Det.A Ch1/254nm

PeakTable

Detector A Ch1 $254 \mathrm{~nm}$
\begin{tabular}{|r|r|r|r|r|r|}
\hline Peak\# & Ret. Time & Area & Height & Area \% & \multicolumn{1}{c|}{ Height \% } \\
\hline 1 & 10.082 & 12547657 & 391015 & 52.106 & 72.759 \\
\hline 2 & 20.303 & 11533222 & 146397 & 47.894 & 27.241 \\
\hline Total & & 24080880 & 537412 & 100.000 & 100.000 \\
\hline
\end{tabular}

$\mathrm{mV}$

D:Icylwglwg-8lwg-10-5-4a OD-H 20\%1.Icd

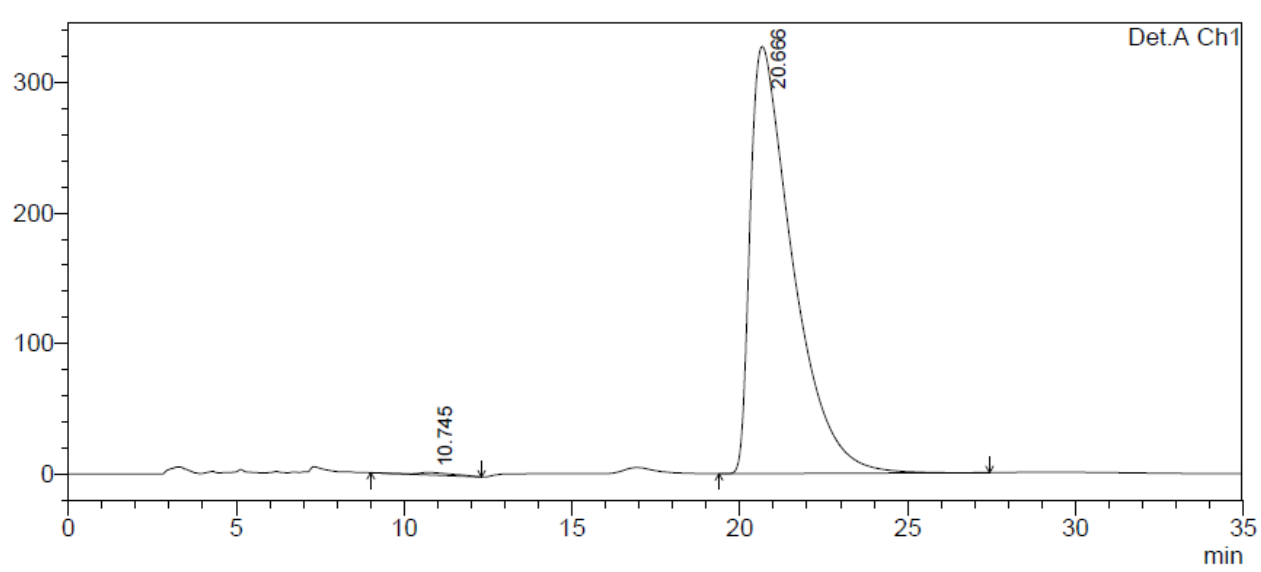

1 Det.A Ch1/254nm

PeakTable

Detector A Ch1 254nm
\begin{tabular}{|r|r|r|r|r|r|}
\hline \multicolumn{1}{|c|}{ Peak\# } & Ret. Time & Area & Height & Area $\%$ & Height $\%$ \\
\hline 1 & 10.745 & 137560 & 2011 & 0.476 & 0.611 \\
\hline 2 & 20.666 & 28776450 & 327060 & 99.524 & 99.389 \\
\hline Total & & 28914010 & 329071 & 100.000 & 100.000 \\
\hline
\end{tabular}




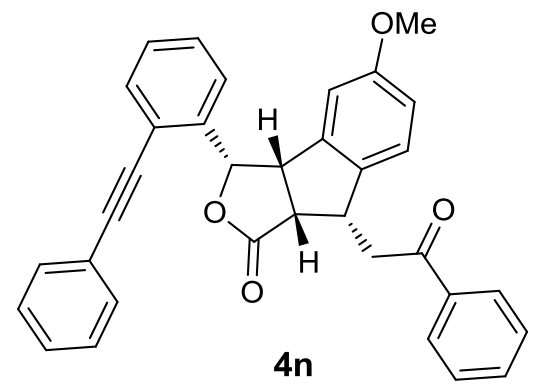

HPLC date of compound 4n: OD-H column, $80: 20$ hexane : IPA, flow rate: $1.0 \mathrm{ml} / \mathrm{min}, 254 \mathrm{~nm}, 25^{\circ} \mathrm{C},>99 \%$ ee.

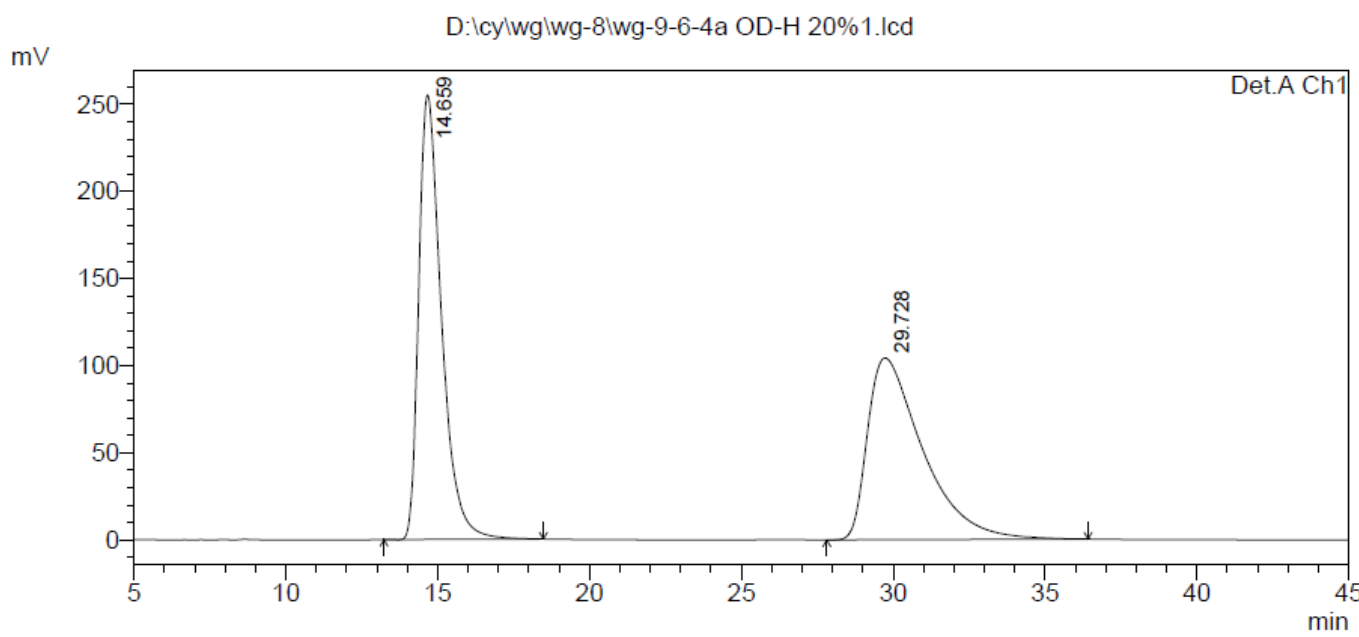

1 Det.A Ch1/254nm

PeakTable

Detector A Ch1 $254 \mathrm{~nm}$
\begin{tabular}{|r|r|r|r|r|r|}
\hline Peak\# & Ret. Time & Area & \multicolumn{1}{c|}{ Height } & \multicolumn{1}{c|}{ Area \% } & \multicolumn{1}{c|}{ Height $\%$} \\
\hline 1 & 14.659 & 13268857 & 254981 & 50.130 & 70.969 \\
\hline 2 & 29.728 & 13199990 & 104307 & 49.870 & 29.031 \\
\hline Total & & 26468846 & 359288 & 100.000 & 100.000 \\
\hline
\end{tabular}

$\mathrm{mV}$

D:Icylwglwg-8lwg-10-6-4a OD-H 20\%1.Icd

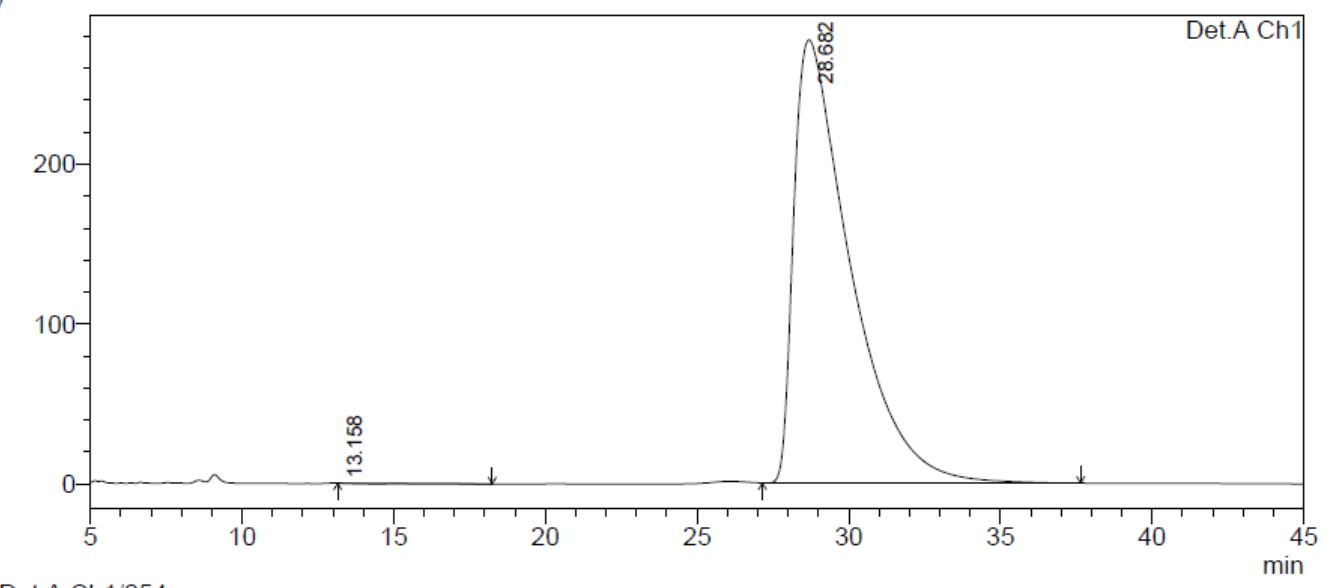

1 Det.A Ch1/254nm

PeakTable

Detector A Ch1 254nm
\begin{tabular}{|r|r|r|r|r|r|}
\hline Peak\# & Ret. Time & Area & Height & Area $\%$ & \multicolumn{1}{|c|}{ Height $\%$} \\
\hline 1 & 13.158 & -48606 & -2 & -0.135 & -0.001 \\
\hline 2 & 28.682 & 35943995 & 277204 & 100.135 & 100.001 \\
\hline Total & & 35895389 & 277203 & 100.000 & 100.000 \\
\hline
\end{tabular}




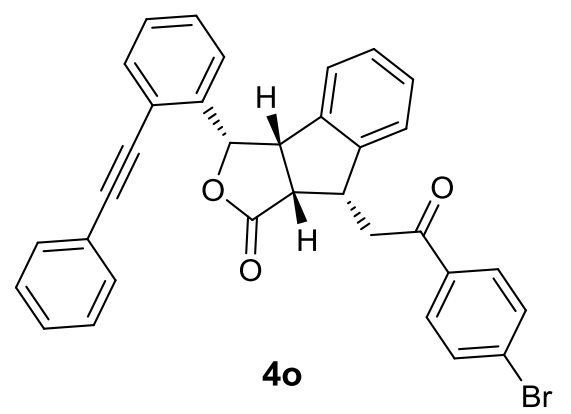

HPLC date of compound 4o: AD-H column, 80:20 hexane : IPA, flow rate: $1.0 \mathrm{ml} / \mathrm{min}, 254 \mathrm{~nm}, 25^{\circ} \mathrm{C}, 99 \%$ ee.

$\mathrm{mV}$

D: Icylwglwg-8lwg-9-7-4a AD-H 20\%1.lcd

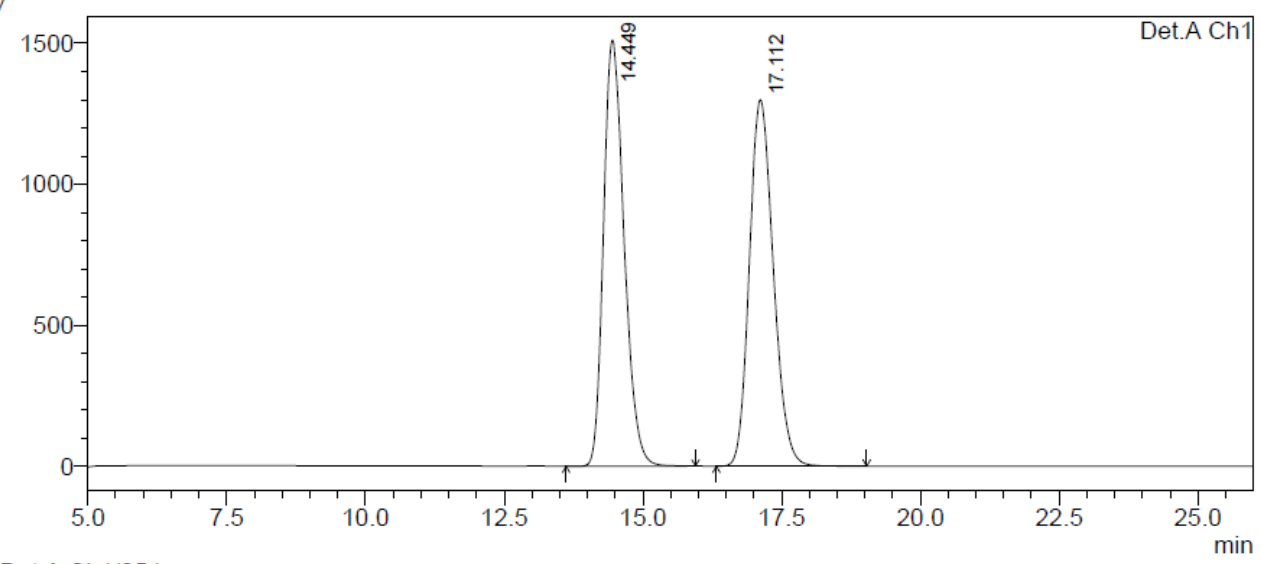

1 Det.A Ch $1 / 254 \mathrm{~nm}$

Detector A Ch1 254nm
\begin{tabular}{|r|r|r|r|r|r|}
\hline Peak\# & Ret. Time & Area & Height & Area $\%$ & \multicolumn{1}{c|}{ Height $\%$} \\
\hline 1 & 14.449 & 39022675 & 1510792 & 49.985 & 53.757 \\
\hline 2 & 17.112 & 39046067 & 1299628 & 50.015 & 46.243 \\
\hline Total & & 78068742 & 2810421 & 100.000 & 100.000 \\
\hline
\end{tabular}

$\mathrm{mV}$

D:lcylwglwg-8lwg-10-7-4a AD-H 20\%1.Icd

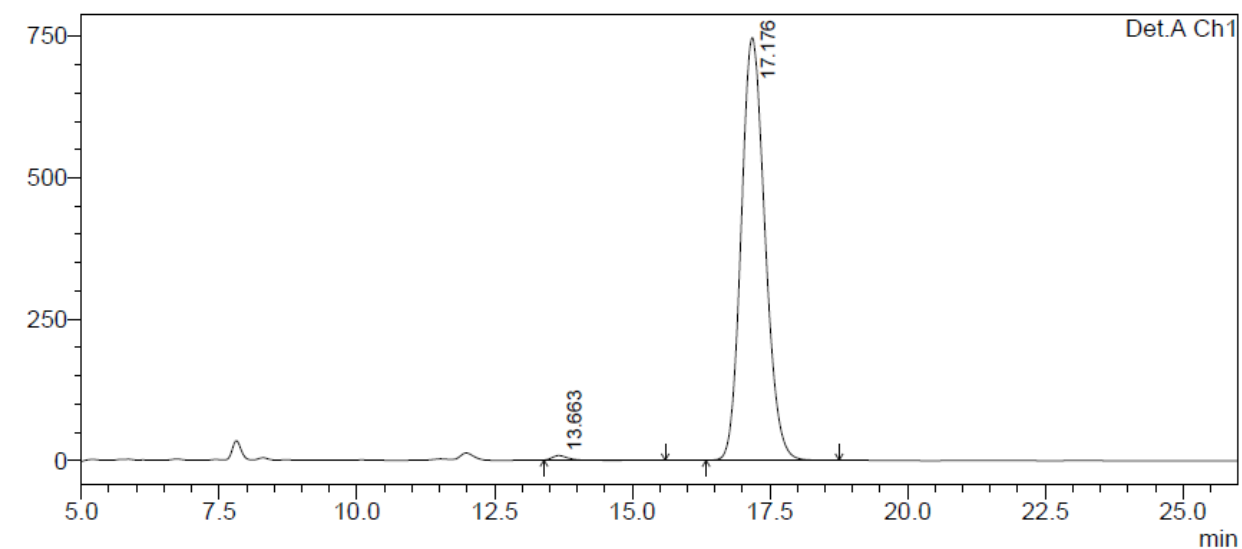

1 Det.A Ch $1 / 254 \mathrm{~nm}$

PeakTable

Detector A Ch1 254nm
\begin{tabular}{|r|r|r|r|r|r|}
\hline \multicolumn{1}{|c|}{ Peak\# } & Ret. Time & \multicolumn{1}{c|}{ Area } & Height & Area $\%$ & Height $\%$ \\
\hline 1 & 13.663 & 134109 & 7990 & 0.595 & 1.059 \\
\hline 2 & 17.176 & 22391393 & 746212 & 99.405 & 98.941 \\
\hline Total & & 22525502 & 754203 & 100.000 & 100.000 \\
\hline
\end{tabular}




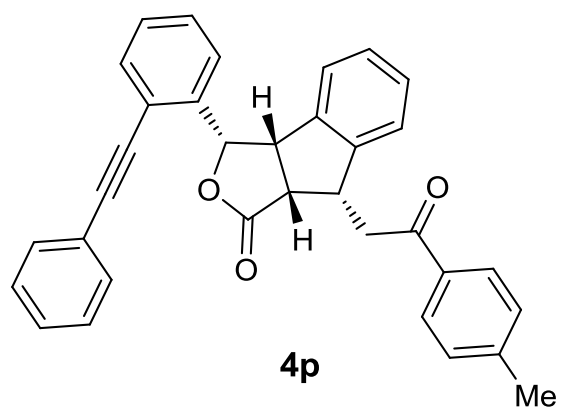

HPLC date of compound 4p: AD-H column, 80:20 hexane : IPA, flow rate: $1.0 \mathrm{ml} / \mathrm{min}, 254 \mathrm{~nm}, 25^{\circ} \mathrm{C},>99 \%$ ee.

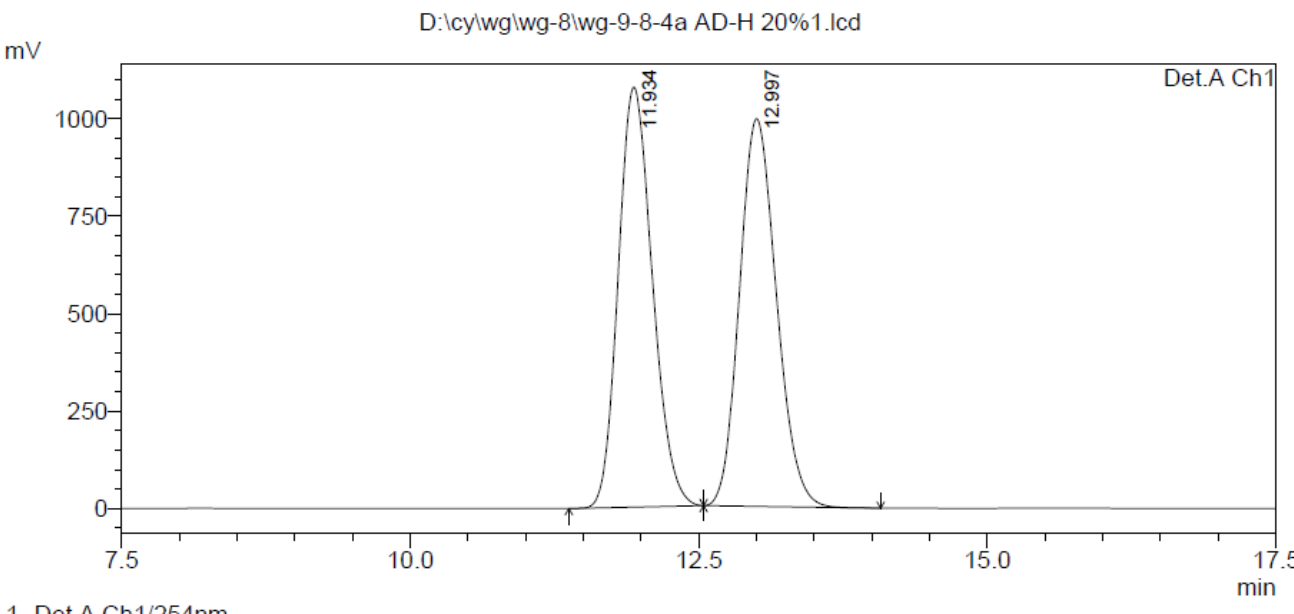

1 Det.A Ch $1 / 254 \mathrm{~nm}$

Detector A Ch1 254nm
\begin{tabular}{|r|r|r|r|r|r|}
\hline Peak\# & Ret. Time & \multicolumn{1}{l}{ Area } & \multicolumn{1}{c|}{ Height } & Area $\%$ & \multicolumn{1}{c|}{ Height $\%$} \\
\hline 1 & 11.934 & 22133442 & 1077153 & 50.010 & 51.990 \\
\hline 2 & 12.997 & 22124345 & 994685 & 49.990 & 48.010 \\
\hline Total & & 44257787 & 2071837 & 100.000 & 100.000 \\
\hline
\end{tabular}

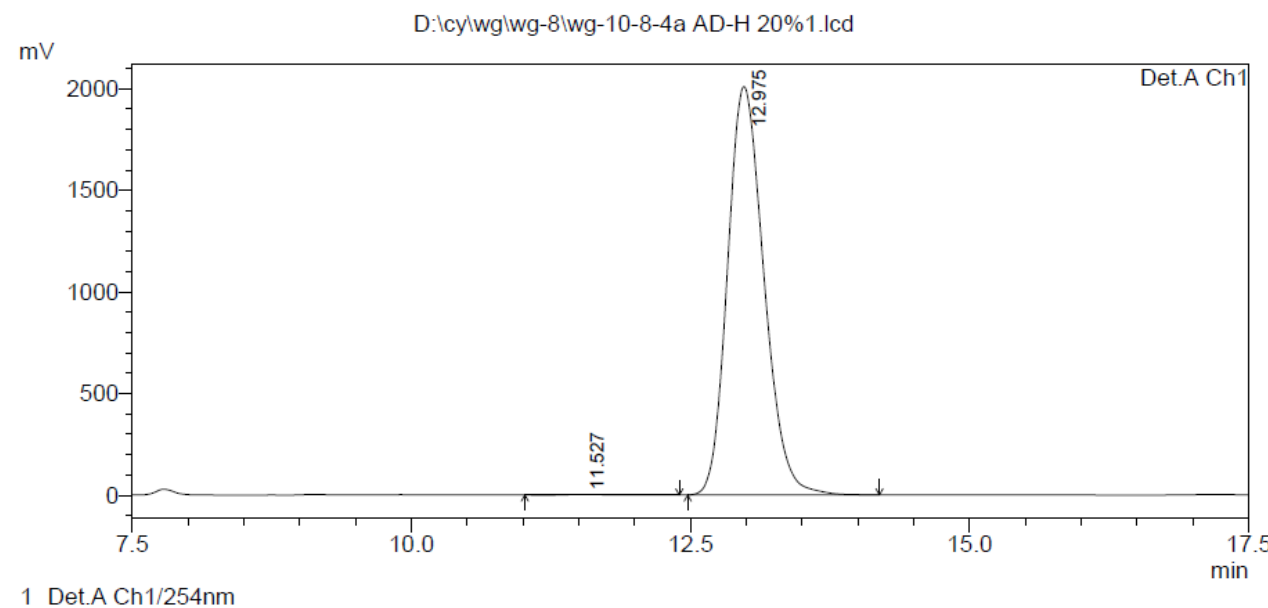

Detector A Ch1 254nm
\begin{tabular}{|r|r|r|r|r|r|}
\hline Peak\# & Ret. Time & Area & Height & \multicolumn{1}{c|}{ Area $\%$} & \multicolumn{1}{c|}{ Height $\%$} \\
\hline 1 & 11.527 & 34569 & 3090 & 0.076 & 0.154 \\
\hline 2 & 12.975 & 45598224 & 2008698 & 99.924 & 99.846 \\
\hline Total & & 45632792 & 2011788 & 100.000 & 100.000 \\
\hline
\end{tabular}




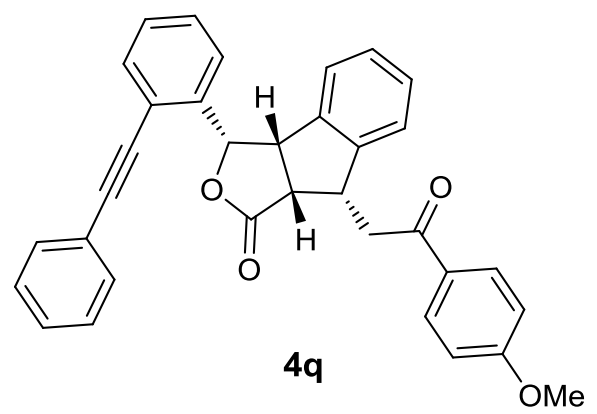

HPLC date of compound 4q: AD-H column, 80:20 hexane : IPA, flow rate: $1.0 \mathrm{ml} / \mathrm{min}, 254 \mathrm{~nm}, 25^{\circ} \mathrm{C},>99 \%$ ee.

$\mathrm{mV}$ D:Icylwglwg-8|wg-9-9-4a AD-H 20\%1.Icd

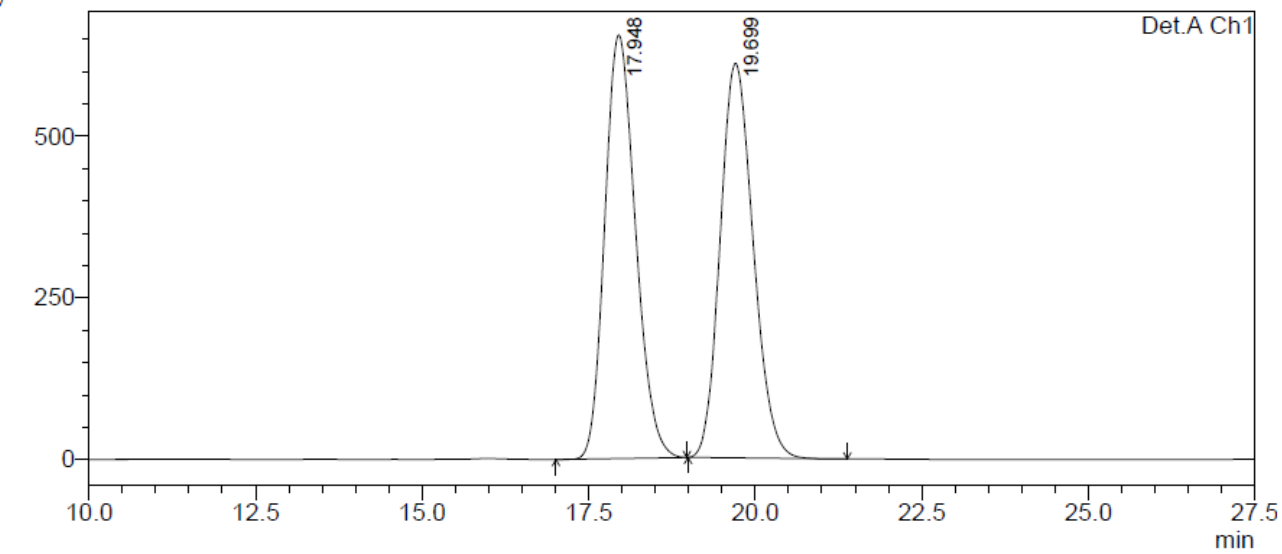

1 Det.A Ch1/254nm

\begin{tabular}{|c|c|c|c|c|c|}
\hline \multicolumn{6}{|c|}{ Detector A Ch1 $254 \mathrm{~nm}$} \\
\hline Peak\# & Ret. Time & Area & Height & Area \% & Height \% \\
\hline 1 & 17.948 & 21035334 & 655011 & 49.987 & 51.7 \\
\hline 2 & 19.699 & 21046396 & 610290 & 50.013 & 48.2 \\
\hline Total & & 42081729 & 1265302 & 100.000 & 100.000 \\
\hline
\end{tabular}

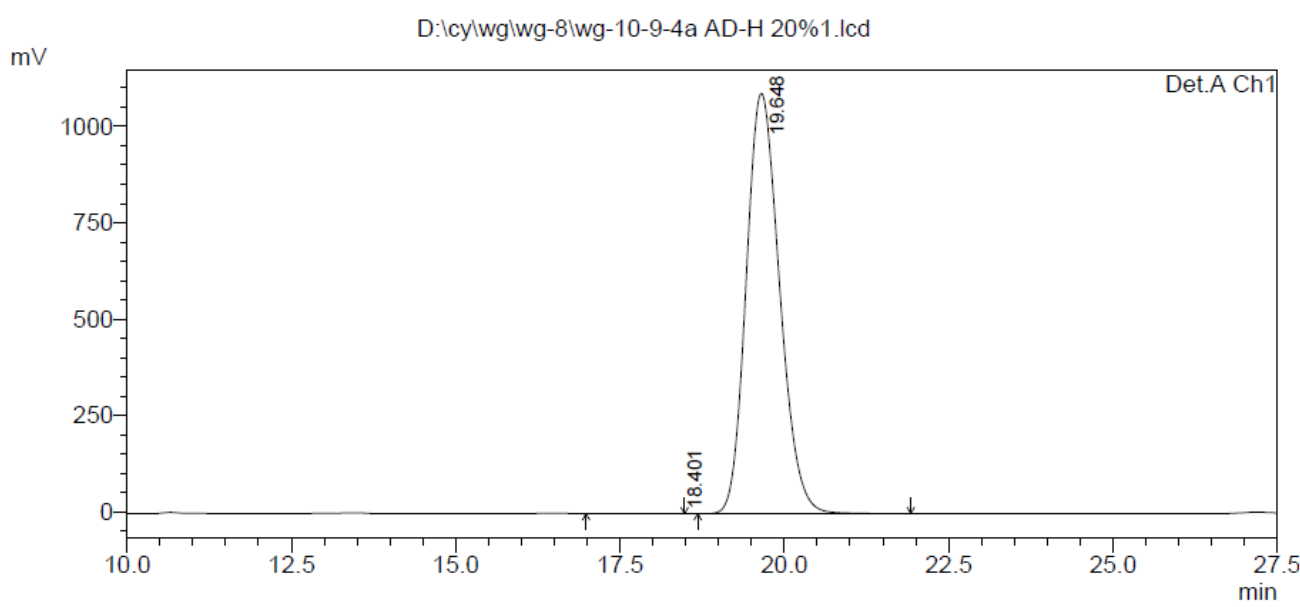

1 Det.A Ch1/254nm

\begin{tabular}{|c|c|c|c|c|c|}
\hline \multicolumn{6}{|c|}{ Detector A Ch1 $254 \mathrm{~nm}$} \\
\hline Peak\# & Ret Time & Area & Height & Area \% & Height \% \\
\hline 1 & 18.401 & -7498 & 15 & -0.019 & 0.001 \\
\hline & 19.648 & 384868 & 1090064 & & 9 \\
\hline Total & & $\begin{array}{l}30+00001 \\
38479304\end{array}$ & 1090079 & 100.000 & 100.000 \\
\hline
\end{tabular}




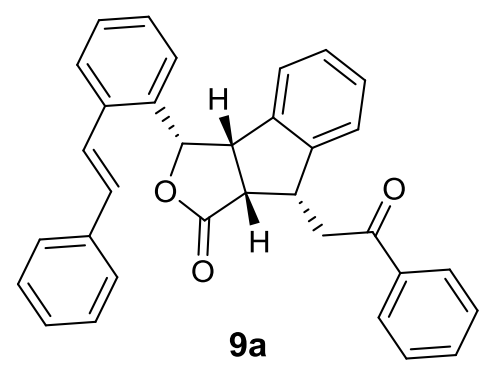

HPLC date of compound 9a: AD-H column, 90:10 hexane : IPA, flow rate: $1.0 \mathrm{ml} / \mathrm{min}, 254 \mathrm{~nm}, 25^{\circ} \mathrm{C},>99 \%$ ee.

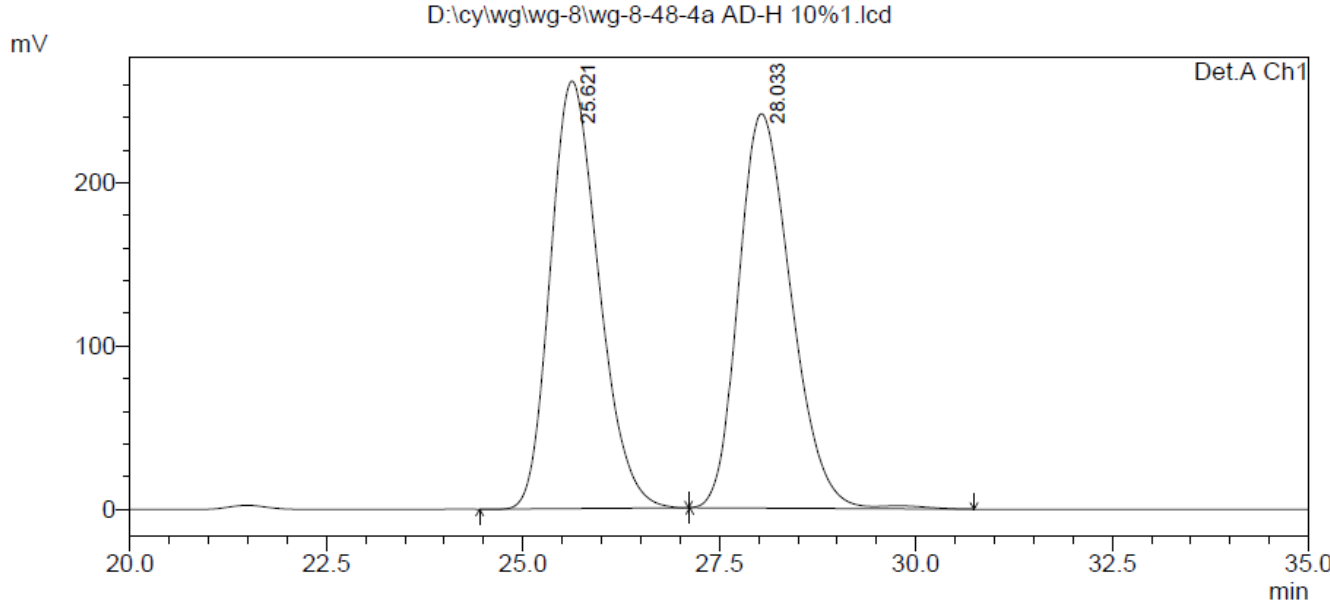

1 Det.A Ch1/254nm

PeakTable

Detector A Ch1 254nm
\begin{tabular}{|r|r|r|r|r|r|}
\hline Peak\# & Ret. Time & \multicolumn{1}{l|}{ Area } & Height & Area $\%$ & Height $\%$ \\
\hline 1 & 25.621 & 11237222 & 261894 & 49.852 & 52.022 \\
\hline 2 & 28.033 & 11303831 & 241533 & 50.148 & 47.978 \\
\hline Total & & 22541052 & 503427 & 100.000 & 100.000 \\
\hline
\end{tabular}

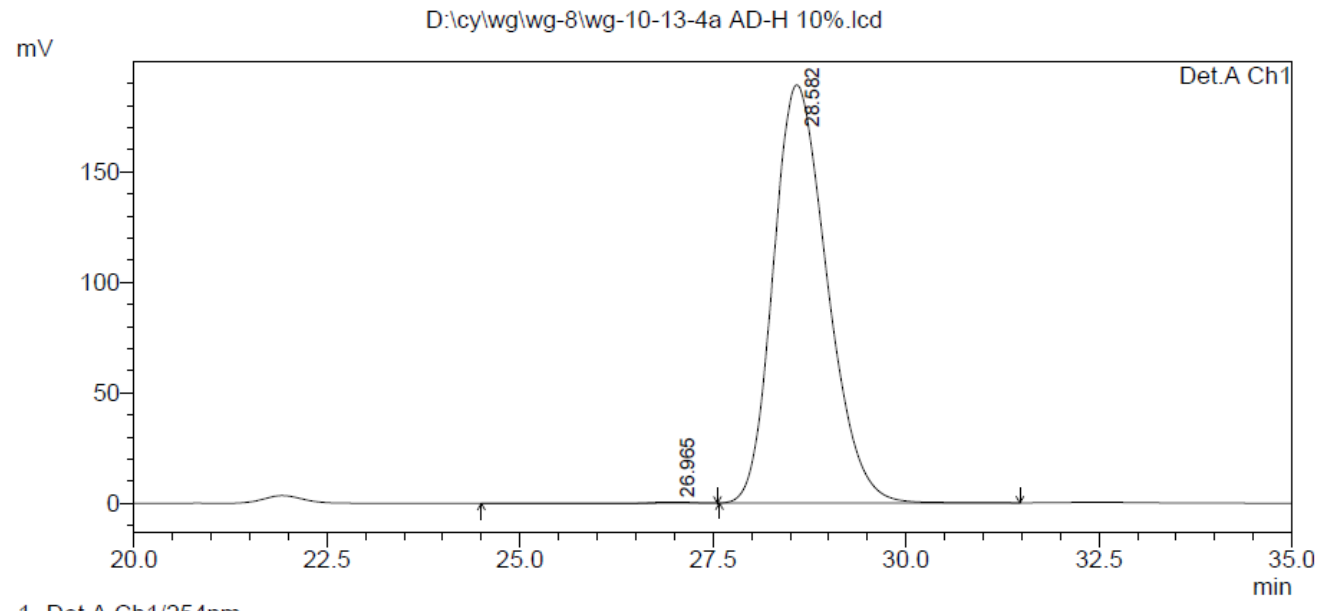

1 Det.A Ch $1 / 254 \mathrm{~nm}$

Detector A Ch1 254nm
\begin{tabular}{|r|r|r|r|r|r|}
\hline Peak\# & Ret. Time & \multicolumn{1}{c|}{ Area } & \multicolumn{1}{c|}{ Height } & \multicolumn{1}{c|}{ Area $\%$} & \multicolumn{1}{c|}{ Height \% } \\
\hline 1 & 26.965 & 8710 & 487 & 0.095 & 0.257 \\
\hline 2 & 28.582 & 9163222 & 189085 & 99.905 & 99.743 \\
\hline Total & & 9171933 & 189572 & 100.000 & 100.000 \\
\hline
\end{tabular}




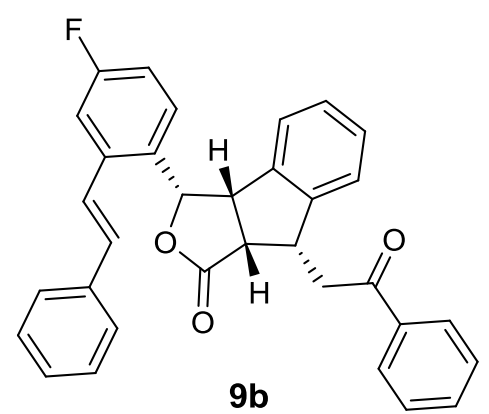

HPLC date of compound 9b: OD-H column, 80:20 hexane : IPA, flow rate: $1.0 \mathrm{ml} / \mathrm{min}, 254 \mathrm{~nm}, 25^{\circ} \mathrm{C},>99 \%$ ee.

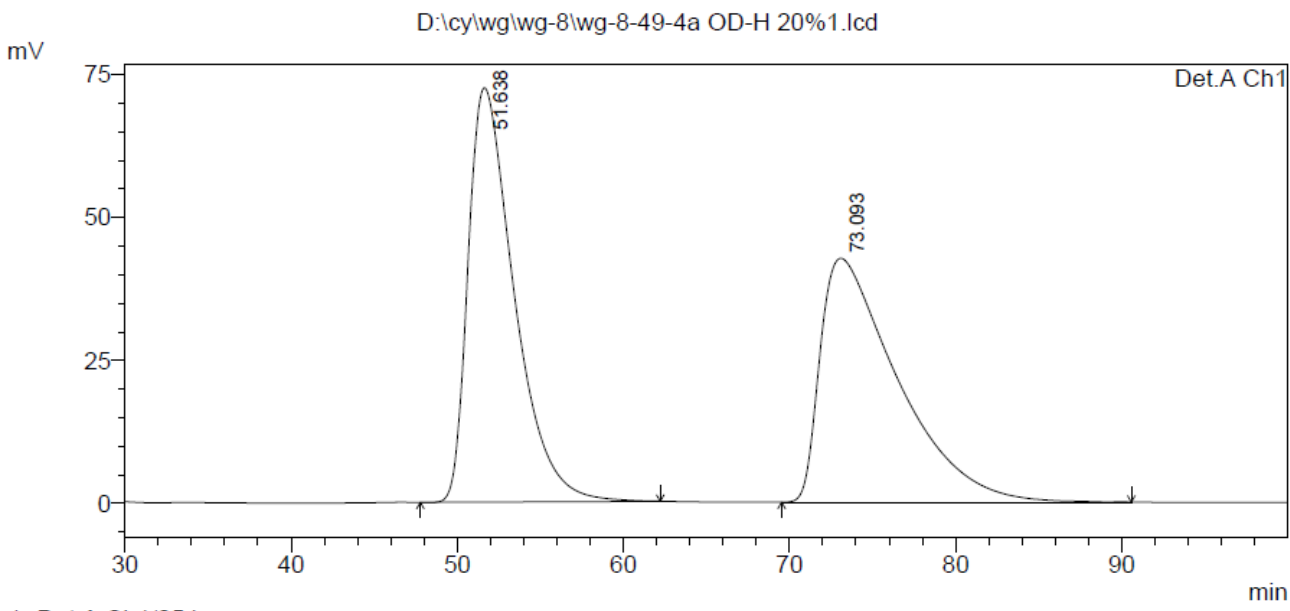

1 Det.A Ch1/254nm

\begin{tabular}{|c|c|c|c|c|c|}
\hline \multirow{2}{*}{\multicolumn{6}{|c|}{ Detector A Ch1 254nm }} \\
\hline & & & & & \\
\hline Peak\# & Ret. Time & Area & Height & Area \% & Height $\%$ \\
\hline 1 & 51.638 & 13953133 & 72468 & 50.206 & 62.952 \\
\hline & 73.093 & 13838745 & 42649 & 49.794 & 37.048 \\
\hline Total & & 27791878 & 115118 & 100.000 & 100.000 \\
\hline
\end{tabular}

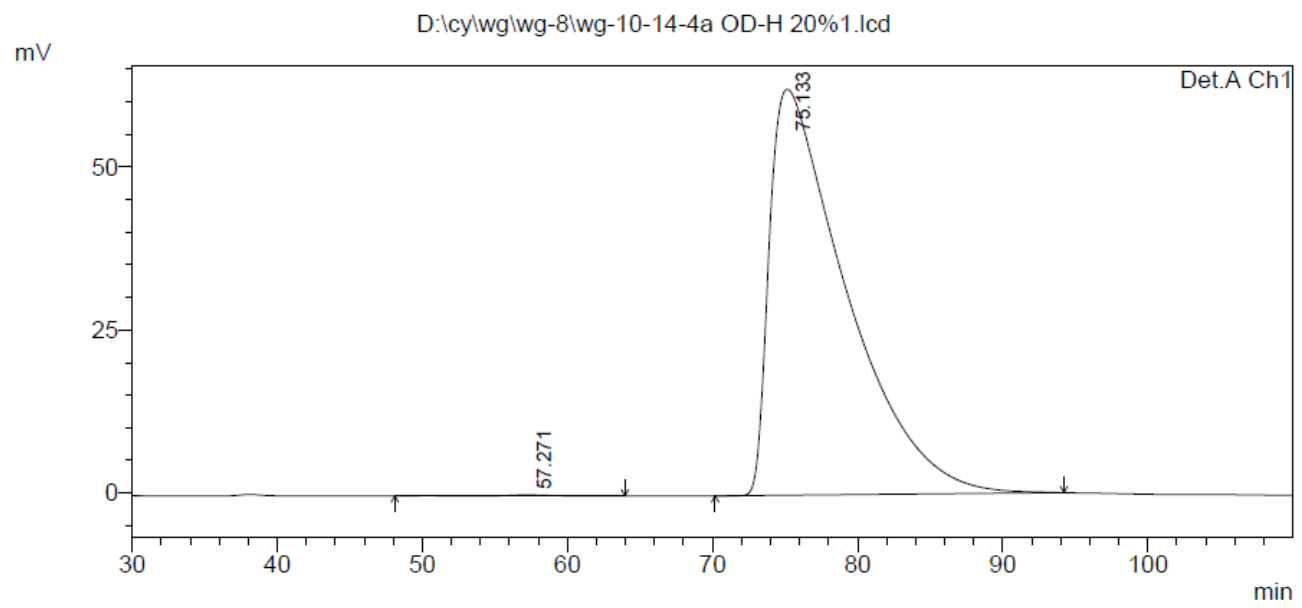

1 Det.A Ch $1 / 254 \mathrm{~nm}$

PeakTable

Detector A Ch1 $254 \mathrm{~nm}$
\begin{tabular}{|r|r|r|r|r|r|}
\hline Peak\# & Ret. Time & \multicolumn{1}{c|}{ Area } & \multicolumn{1}{c|}{ Height } & Area $\%$ & \multicolumn{1}{c|}{ Height $\%$} \\
\hline 1 & 57.271 & 26613 & 172 & 0.116 & 0.275 \\
\hline 2 & 75.133 & 22950956 & 62274 & 99.884 & 99.725 \\
\hline Total & & 22977570 & 62446 & 100.000 & 100.000 \\
\hline
\end{tabular}




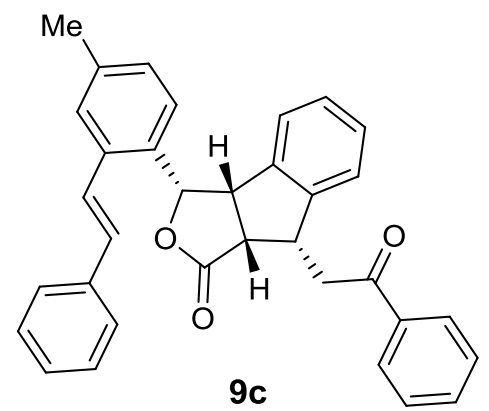

HPLC date of compound 9c: OD-H column, 80:20 hexane : IPA, flow rate: $1.0 \mathrm{ml} / \mathrm{min}, 254 \mathrm{~nm}, 25^{\circ} \mathrm{C},>99 \%$ ee.

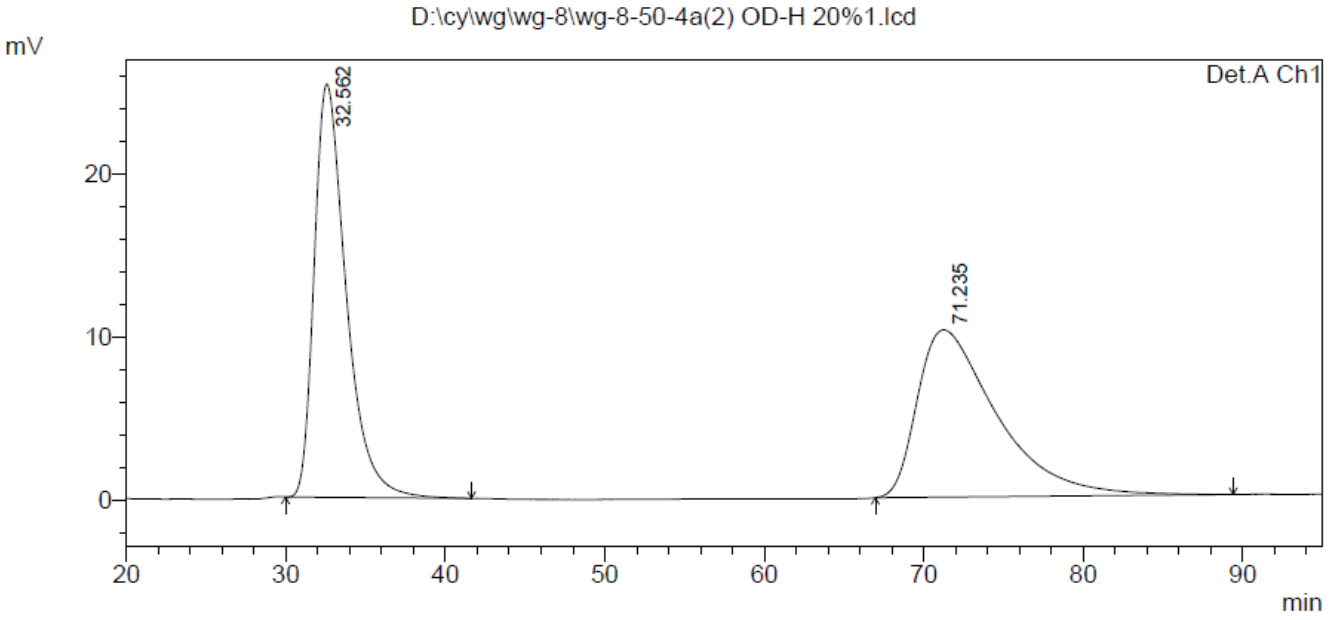

1 Det.A Ch1/254nm

Detector A Ch1 $254 \mathrm{~nm}$
\begin{tabular}{|r|r|r|r|r|r|}
\hline Peak\# & Ret. Time & Area & Height & Area \% & \multicolumn{1}{|c|}{ Height \% } \\
\hline 1 & 32.562 & 3527507 & 25326 & 49.845 & 71.199 \\
\hline 2 & 71.235 & 3549406 & 10245 & 50.155 & 28.801 \\
\hline Total & & 7076913 & 35571 & 100.000 & 100.000 \\
\hline
\end{tabular}

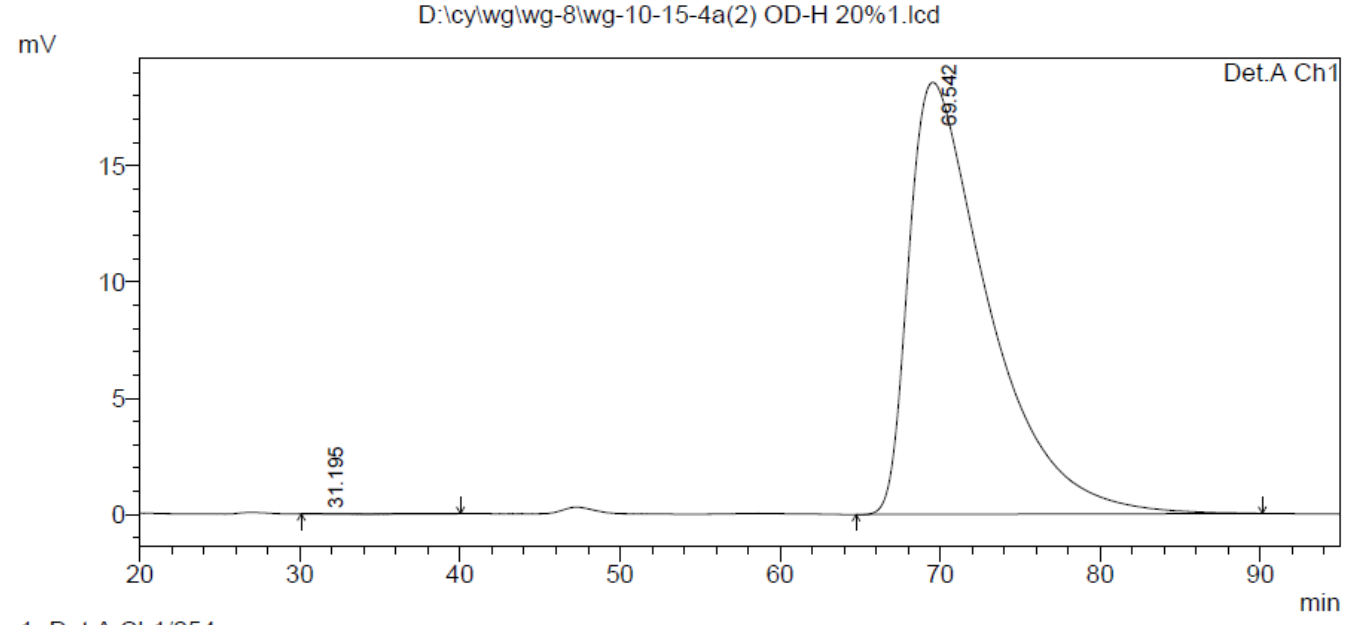

1 Det.A Ch1/254nm

Detector A Ch1 254nm
\begin{tabular}{|r|r|r|r|r|r|}
\hline Peak\# & Ret. Time & Area & Height & \multicolumn{1}{c|}{ Area \% } & \multicolumn{1}{c|}{ Height \% } \\
\hline 1 & 31.195 & -6201 & 7 & -0.096 & 0.036 \\
\hline 2 & 69.542 & 6438999 & 18582 & 100.096 & 99.964 \\
\hline Total & & 6432798 & 18589 & 100.000 & 100.000 \\
\hline
\end{tabular}




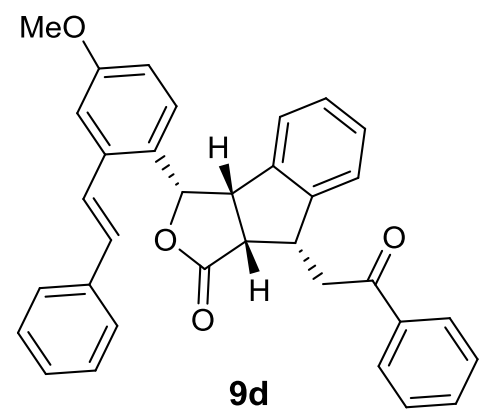

HPLC date of compound 9d: AD-H column, 80:20 hexane : IPA, flow rate: $1.0 \mathrm{ml} / \mathrm{min}, 254 \mathrm{~nm}, 25^{\circ} \mathrm{C},>99 \%$ ee.

$\mathrm{mV}$

D:Icylwglwg-8lwg-8-51-4a AD-H 20\%1.lcd

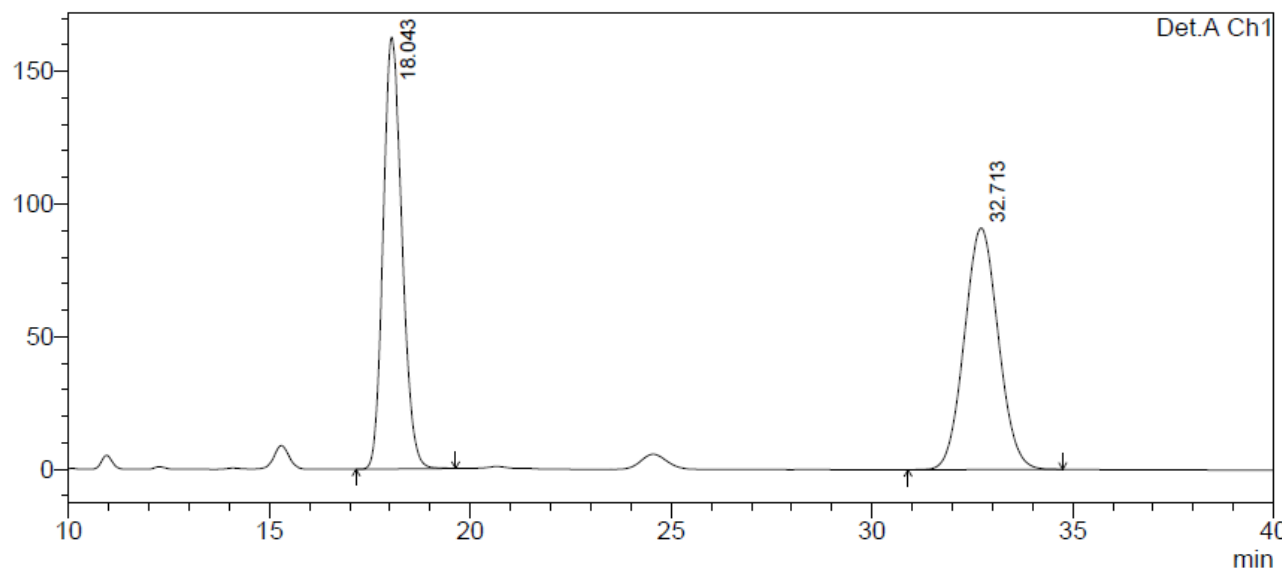

1 Det.A Ch1/254nm

Detector A Ch1 254nm
\begin{tabular}{|r|r|r|r|r|r|}
\hline \multicolumn{1}{|c|}{ Peak\# } & Ret. Time & \multicolumn{1}{c|}{ Area } & Height & Area \% & \multicolumn{1}{c|}{ Height \% } \\
\hline 1 & 18.043 & 5297401 & 162623 & 49.703 & 64.121 \\
\hline 2 & 32.713 & 5360779 & 90995 & 50.297 & 35.879 \\
\hline Total & & 10658180 & 253617 & 100.000 & 100.000 \\
\hline
\end{tabular}

$\mathrm{mV}$

D:lcylwglwg-8lwg-10-16-4a AD-H 20\%.Icd

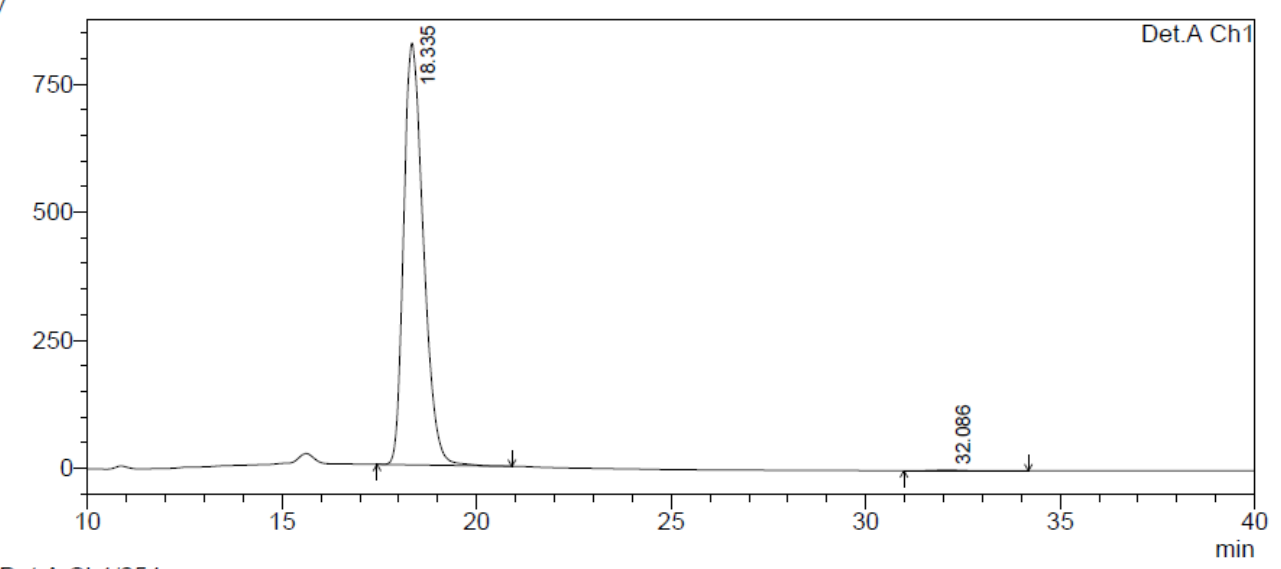

1 Det.A Ch1/254nm

PeakTable
\begin{tabular}{|r|r|r|r|r|r|} 
Detector A Ch1 254nm \\
\hline Peak\# & Ret. Time & \multicolumn{1}{|c|}{ Area } & \multicolumn{1}{c|}{ Height } & \multicolumn{1}{c|}{ Area \% } & \multicolumn{1}{c|}{ Height \% } \\
\hline 1 & 18.335 & 29081138 & 823231 & 99.743 & 99.833 \\
\hline 2 & 32.086 & 74915 & 1377 & 0.257 & 0.167 \\
\hline Total & & 29156053 & 824608 & 100.000 & 100.000 \\
\hline
\end{tabular}




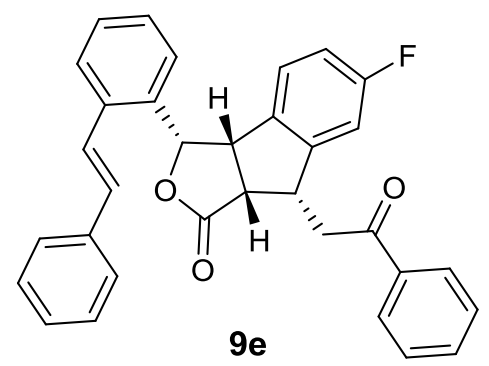

HPLC date of compound 9e: AD-H column, 80:20 hexane : IPA, flow rate: $1.0 \mathrm{ml} / \mathrm{min}, 254 \mathrm{~nm}, 25^{\circ} \mathrm{C}, 99 \%$ ee.

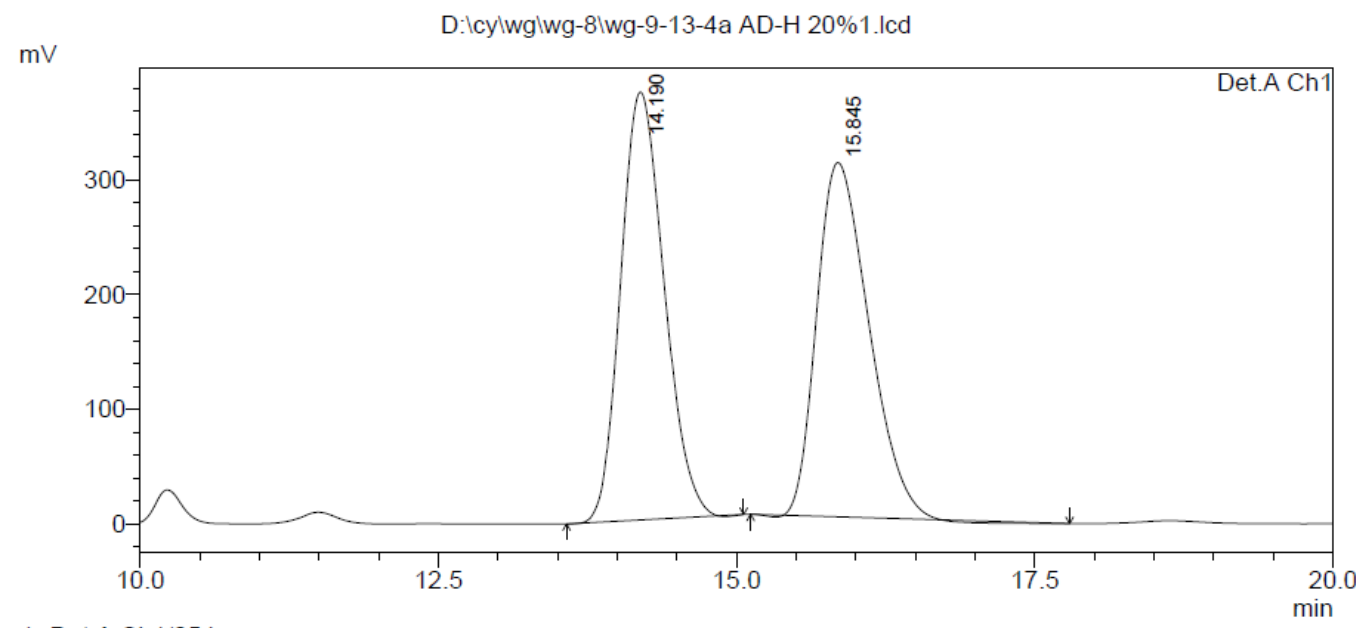

1 Det.A Ch1/254nm

PeakTable
\begin{tabular}{|r|r|r|r|r|r|}
\hline Detector A Ch1 254nm \\
\hline Peak\# & Ret. Time & Area & Height & Area \% & \multicolumn{1}{c|}{ Height \% } \\
\hline 1 & 14.190 & 9449475 & 373187 & 50.321 & 54.719 \\
\hline 2 & 15.845 & 9329039 & 308814 & 49.679 & 45.281 \\
\hline Total & & 18778513 & 682002 & 100.000 & 100.000 \\
\hline
\end{tabular}

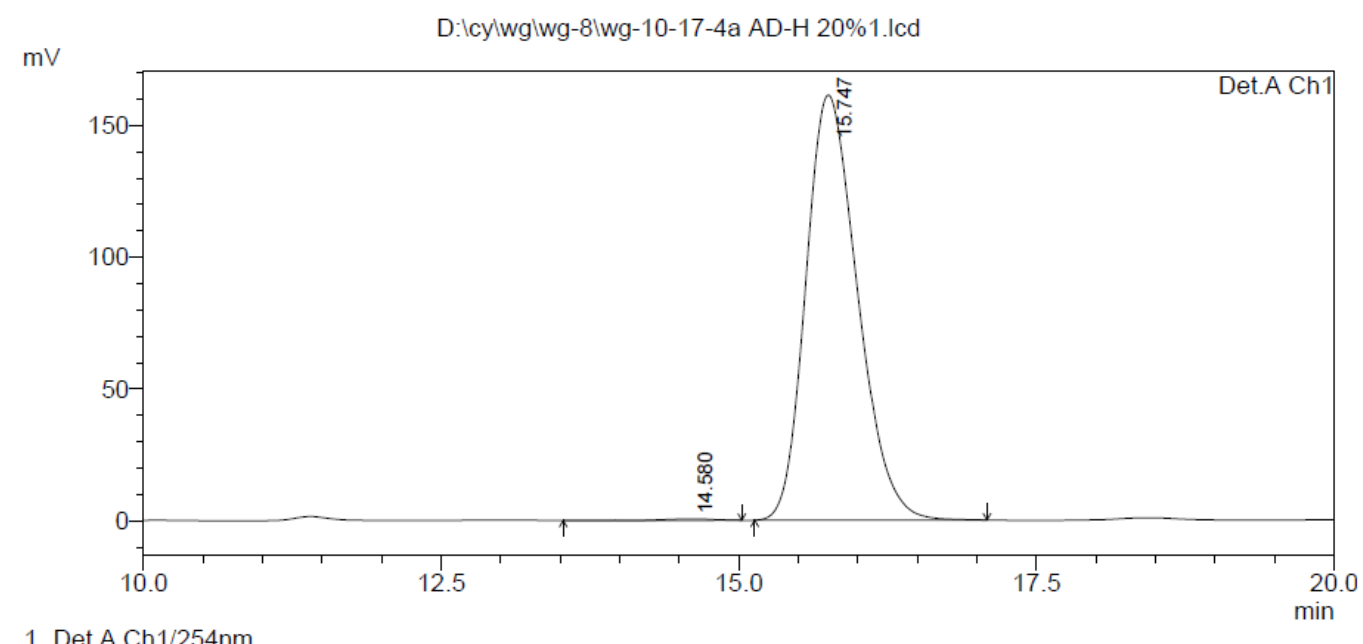

1 Det.A Ch1/254nm

PeakTable

Detector A Ch1 $254 \mathrm{~nm}$
\begin{tabular}{|r|r|r|r|r|r|}
\hline Peak\# & Ret. Time & \multicolumn{1}{|c|}{ Area } & Height & Area $\%$ & Height $\%$ \\
\hline 1 & 14.580 & 15255 & 555 & 0.312 & 0.343 \\
\hline 2 & 15.747 & 4869792 & 161273 & 99.688 & 99.657 \\
\hline Total & & 4885047 & 161828 & 100.000 & 100.000 \\
\hline
\end{tabular}




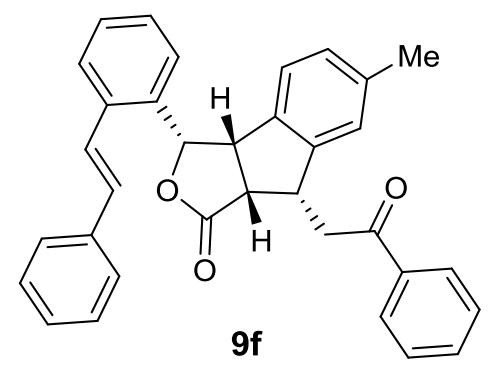

HPLC date of compound 9f: OD-H column, $80: 20$ hexane : IPA, flow rate: $1.0 \mathrm{ml} / \mathrm{min}, 254 \mathrm{~nm}, 25^{\circ} \mathrm{C}, 99 \%$ ee.

$\mathrm{mV}$

D:lcylwg lwg-8lwg-9-14-4a OD-H 20\%1.Icd

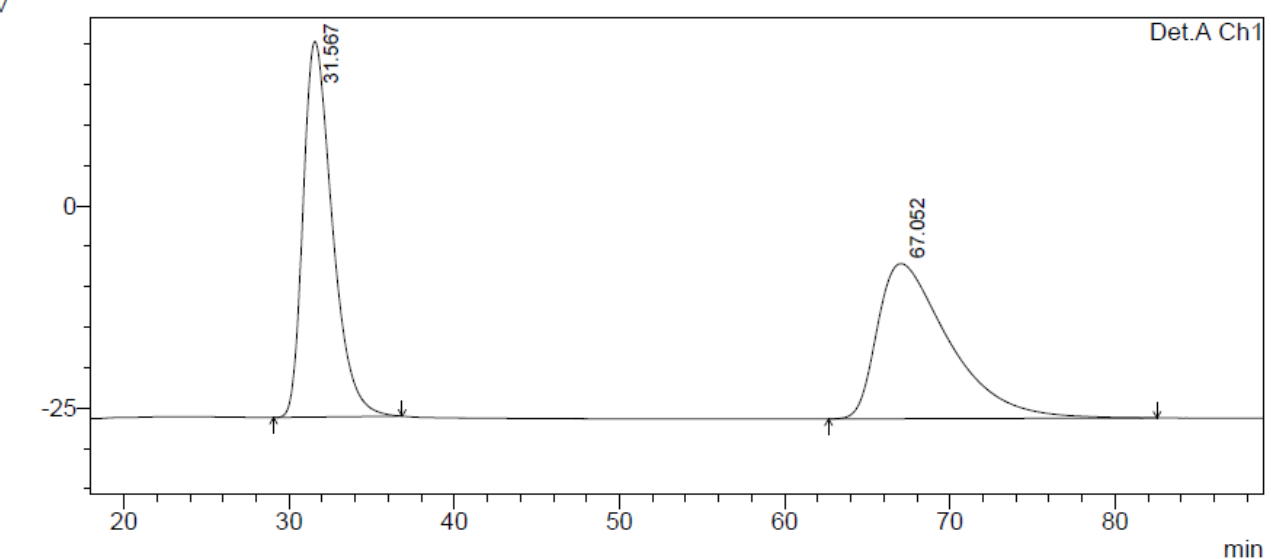

1 Det.A Ch1/254nm

Detector A Ch1 254nm
\begin{tabular}{|r|r|r|r|r|r|}
\hline Peak\# & Ret. Time & Area & Height & \multicolumn{1}{c|}{ Area \% } & \multicolumn{1}{c|}{ Height \% } \\
\hline 1 & 31.567 & 5732497 & 46525 & 49.695 & 70.785 \\
\hline 2 & 67.052 & 5802832 & 19203 & 50.305 & 29.215 \\
\hline Total & & 11535330 & 65728 & 100.000 & 100.000 \\
\hline
\end{tabular}

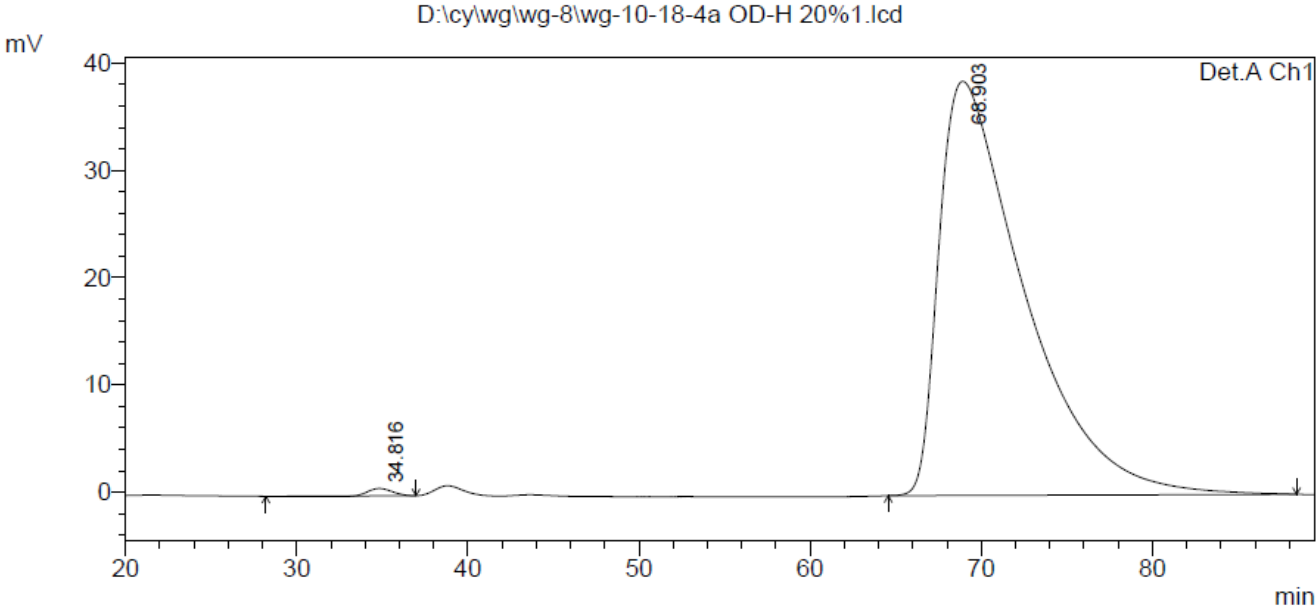

1 Det.A Ch1/254nm

PeakTable

Detector A Ch1 254nm
\begin{tabular}{|r|r|r|r|r|r|}
\hline Peak\# & Ret. Time & \multicolumn{1}{c|}{ Area } & Height & Area $\%$ & \multicolumn{1}{|c|}{ Height $\%$} \\
\hline 1 & 34.816 & 61649 & 697 & 0.458 & 1.772 \\
\hline 2 & 68.903 & 13403558 & 38611 & 99.542 & 98.228 \\
\hline Total & & 13465206 & 39308 & 100.000 & 100.000 \\
\hline
\end{tabular}




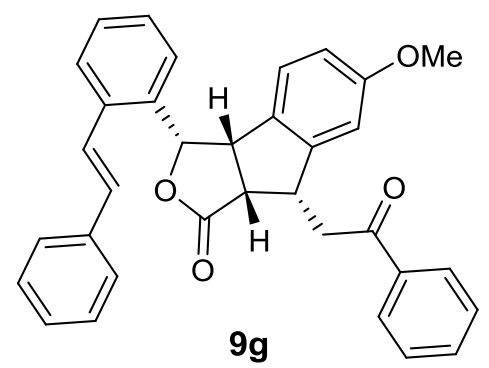

HPLC date of compound 9g: AD-H column, $80: 20$ hexane : IPA, flow rate: $1.0 \mathrm{ml} / \mathrm{min}, 254 \mathrm{~nm}, 25^{\circ} \mathrm{C}, 99 \%$ ee.

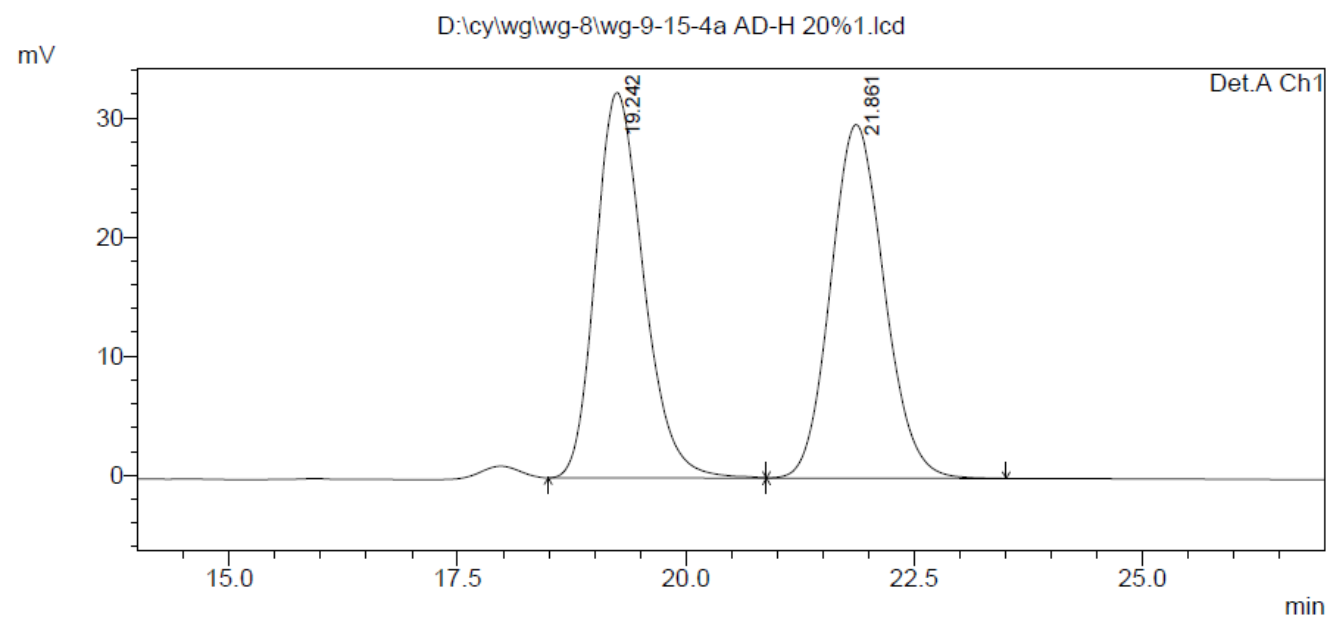

1 Det.A Ch1/254nm

Detector A Ch1 254nm
\begin{tabular}{|r|r|r|r|r|r|}
\hline Peak\# & Ret. Time & Area & Height & \multicolumn{1}{c|}{ Area \% } & \multicolumn{1}{c|}{ Height \% } \\
\hline 1 & 19.242 & 1185600 & 32354 & 49.419 & 52.147 \\
\hline 2 & 21.861 & 1213491 & 29691 & 50.581 & 47.853 \\
\hline Total & & 2399091 & 62045 & 100.000 & 100.000 \\
\hline
\end{tabular}

$\mathrm{mV}$

D:Icylwglwg-8|wg-10-19-4a AD-H 20\%1.lcd

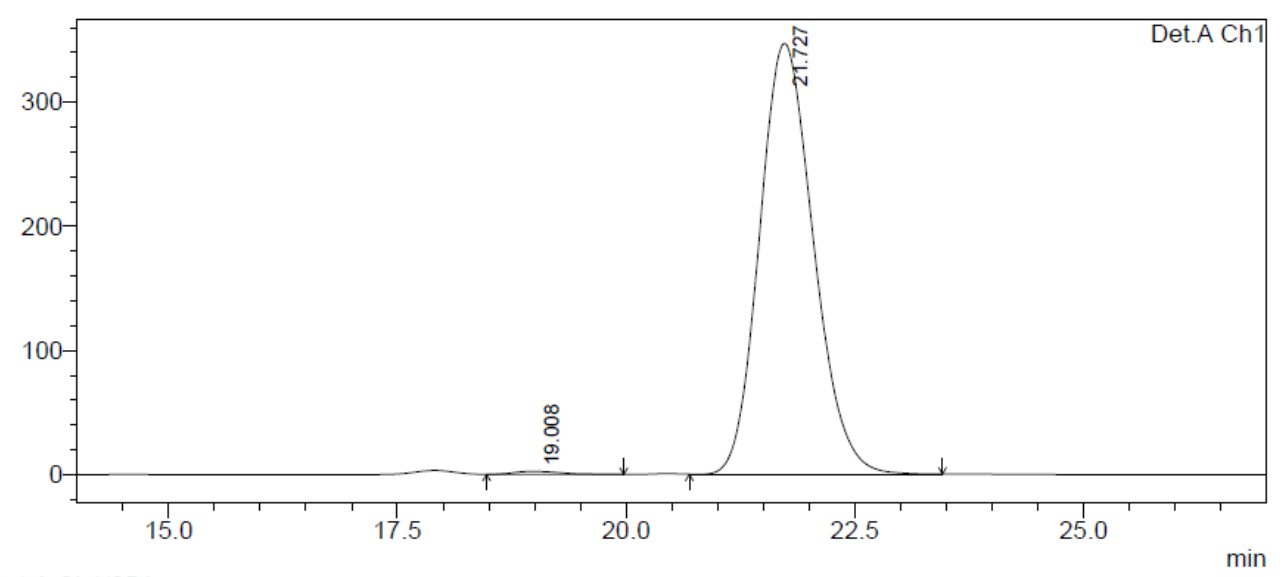

1 Det.A Ch1/254nm

PeakTable

Detector A Ch1 $254 \mathrm{~nm}$
\begin{tabular}{|r|r|r|r|r|r|}
\hline Peak\# & Ret. Time & Area & Height & \multicolumn{1}{c|}{ Area $\%$} & Height $\%$ \\
\hline 1 & 19.008 & 86128 & 2588 & 0.602 & 0.741 \\
\hline 2 & 21.727 & 14225156 & 346870 & 99.398 & 99.259 \\
\hline Total & & 14311285 & 349458 & 100.000 & 100.000 \\
\hline
\end{tabular}




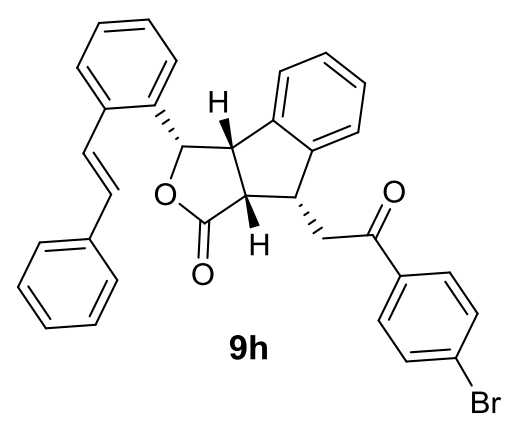

HPLC date of compound 9h: AD-H column, 80:20 hexane : IPA, flow rate: $1.0 \mathrm{ml} / \mathrm{min}, 254 \mathrm{~nm}, 25^{\circ} \mathrm{C}, 97 \%$ ee.

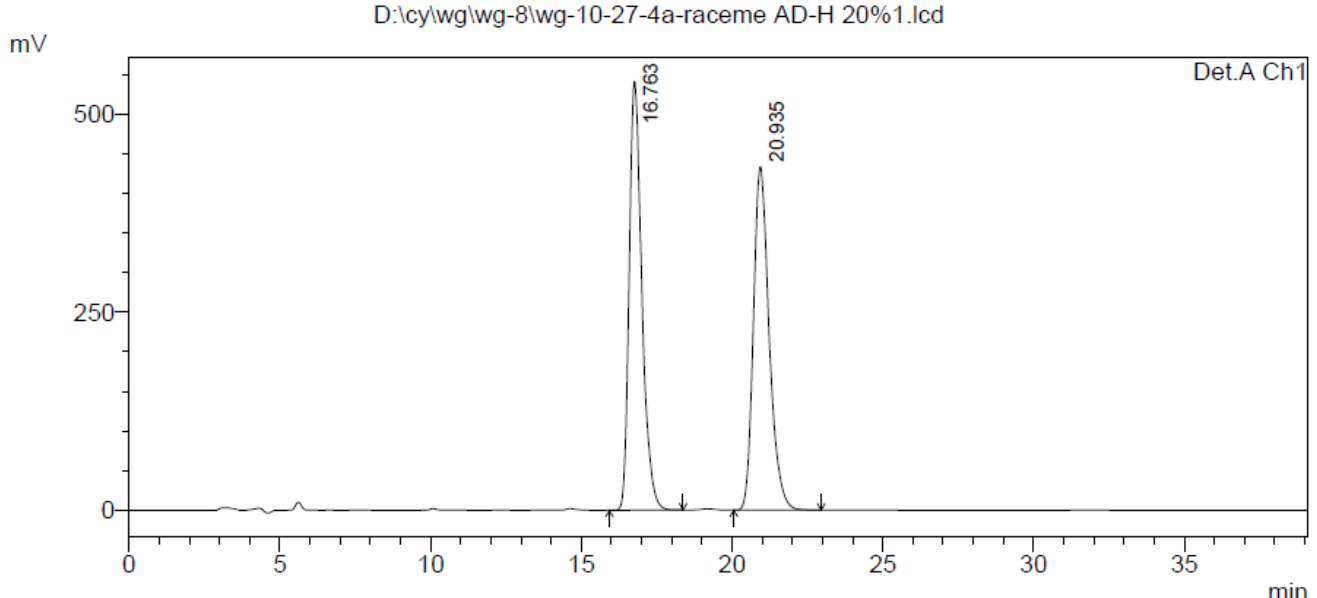

1 Det.A Ch $1 / 254 \mathrm{~nm}$

PeakTable

Detector A Ch1 254nm
\begin{tabular}{|r|r|r|r|r|r|}
\hline Peak\# & Ret. Time & Area & Height & Area \% & Height \% \\
\hline 1 & 16.763 & 15793277 & 541175 & 50.061 & 55.546 \\
\hline 2 & 20.935 & 15754902 & 433105 & 49.939 & 44.454 \\
\hline Total & & 31548179 & 974280 & 100.000 & 100.000 \\
\hline
\end{tabular}

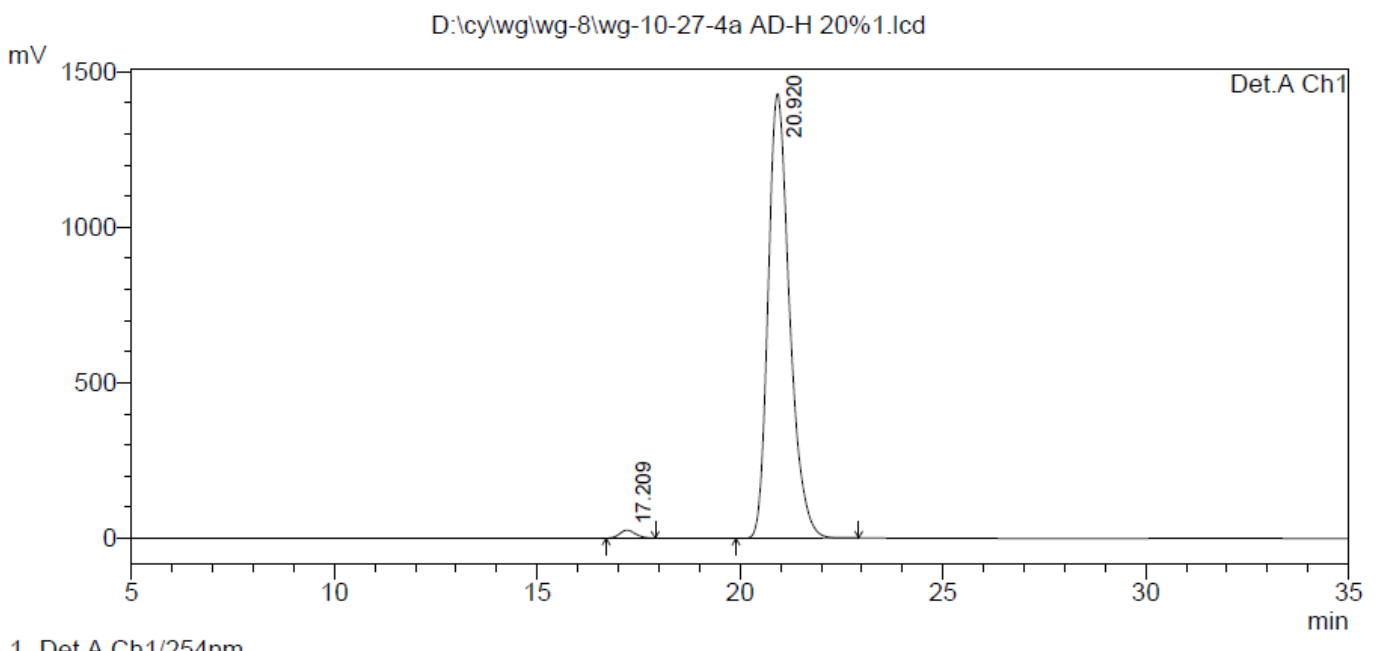

1 Det.A Ch1/254nm

\begin{tabular}{|c|c|c|c|c|c|}
\hline Peak\# & Ret. Time & Area & Height & Area $\%$ & Height \% \\
\hline 1 & 17.209 & 718312 & 25970 & 1.351 & 1.786 \\
\hline 2 & 20.920 & 52440381 & 1428420 & 98.649 & 98.214 \\
\hline Total & & 53158693 & 1454390 & 100.000 & 100.000 \\
\hline
\end{tabular}




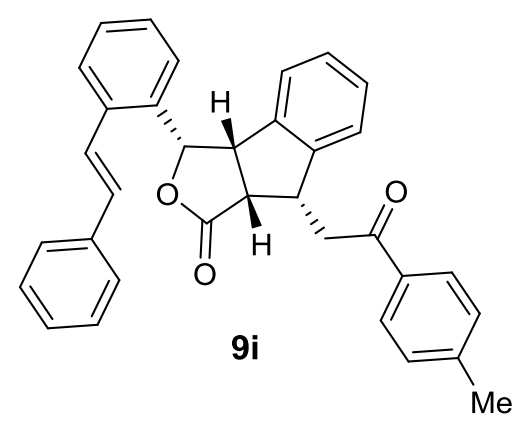

HPLC date of compound 9i: OD-H column, 80:20 hexane : IPA, flow rate: $1.0 \mathrm{ml} / \mathrm{min}, 254 \mathrm{~nm}$, $25^{\circ} \mathrm{C},>99 \%$ ee.

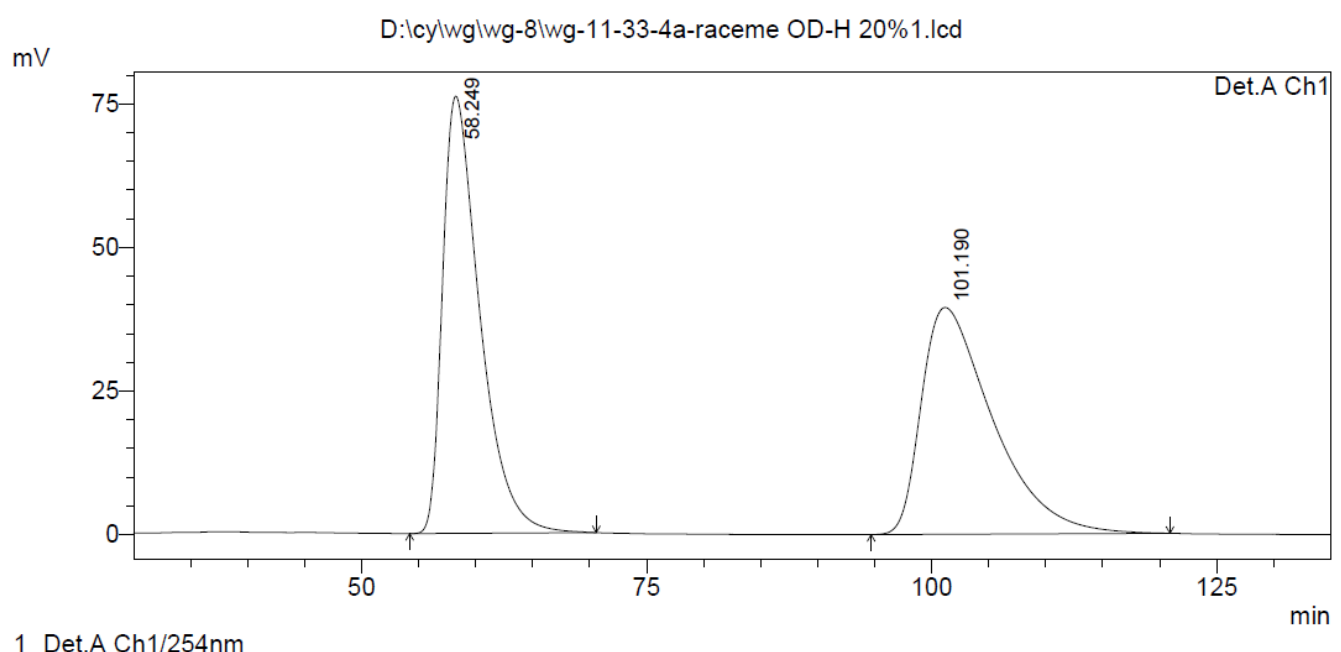

1 Det.A Ch1/254nm

Detector A Ch1 254nm
\begin{tabular}{|r|r|r|r|r|r|}
\hline Peak\# & Ret. Time & Area & Height & Area $\%$ & \multicolumn{1}{|c|}{ Height \% } \\
\hline 1 & 58.249 & 17332519 & 76153 & 50.164 & 65.848 \\
\hline 2 & 101.190 & 17219492 & 39497 & 49.836 & 34.152 \\
\hline Total & & 34552011 & 115650 & 100.000 & 100.000 \\
\hline
\end{tabular}

$\mathrm{mV}$

D:lcylwglwg-8lwg-11-33-4a OD-H 20\%1.lcd

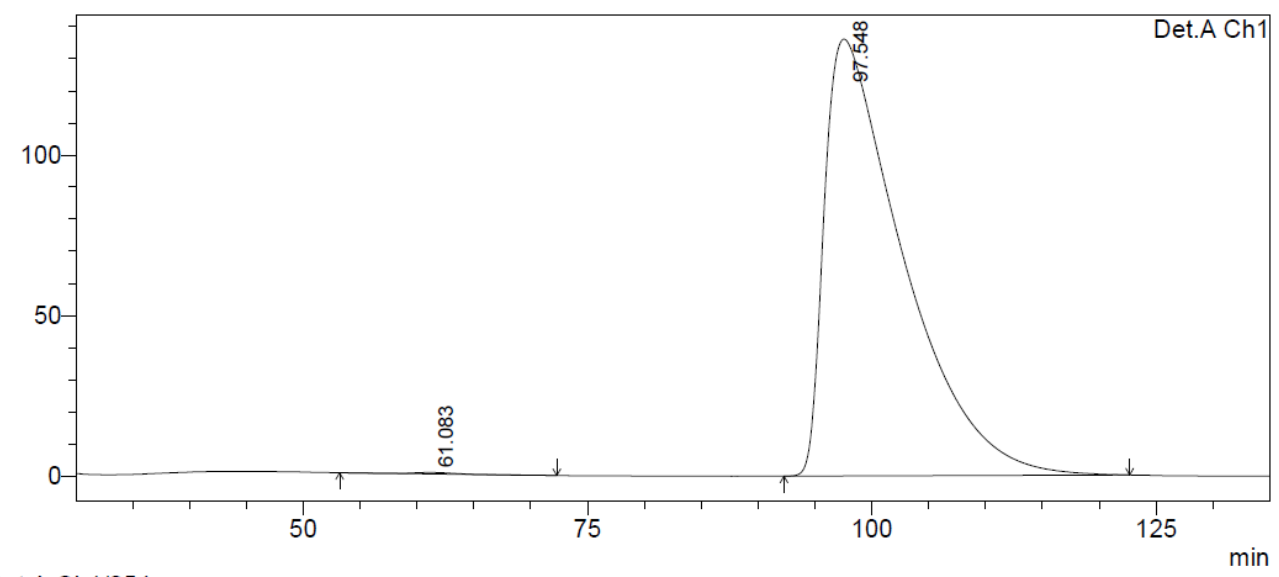

1 Det.A Ch1/254nm

PeakTable

Detector A Ch1 $254 \mathrm{~nm}$
\begin{tabular}{|r|r|r|r|r|r|}
\hline Peak\# & Ret. Time & Area & Height & Area $\%$ & Height $\%$ \\
\hline 1 & 61.083 & 74964 & 446 & 0.114 & 0.326 \\
\hline 2 & 97.548 & 65639341 & 136111 & 99.886 & 99.674 \\
\hline Total & & 65714305 & 136557 & 100.000 & 100.000 \\
\hline
\end{tabular}




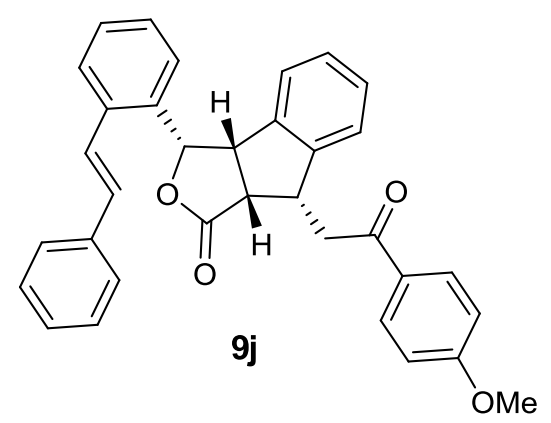

HPLC date of compound 9j: OD-H column, 80:20 hexane : IPA, flow rate: $1.0 \mathrm{ml} / \mathrm{min}, 254 \mathrm{~nm}$, $25^{\circ} \mathrm{C},>99 \%$ ee.

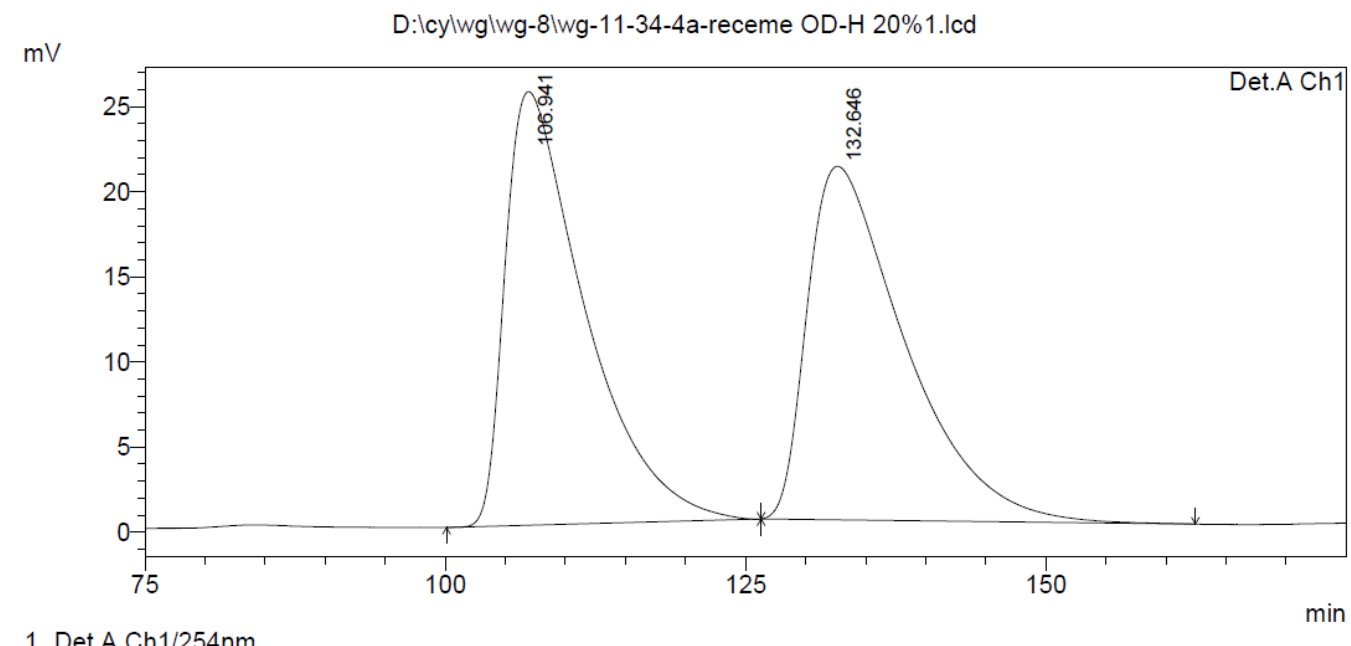

1 Det.A Ch1/254nm

PeakTable

Detector A Ch1 $254 \mathrm{~nm}$
\begin{tabular}{|r|r|r|r|r|r|}
\hline Peak\# & Ret. Time & \multicolumn{1}{l}{ Area } & Height & Area $\%$ & \multicolumn{1}{c|}{ Height $\%$} \\
\hline 1 & 106.941 & 11737696 & 25477 & 49.976 & 55.085 \\
\hline 2 & 132.646 & 11748736 & 20774 & 50.024 & 44.915 \\
\hline Total & & 23486432 & 46250 & 100.000 & 100.000 \\
\hline
\end{tabular}

$\mathrm{mV}$

D:lcylwglwg-8lwg-11-34-4a OD-H 20\%1.lcd

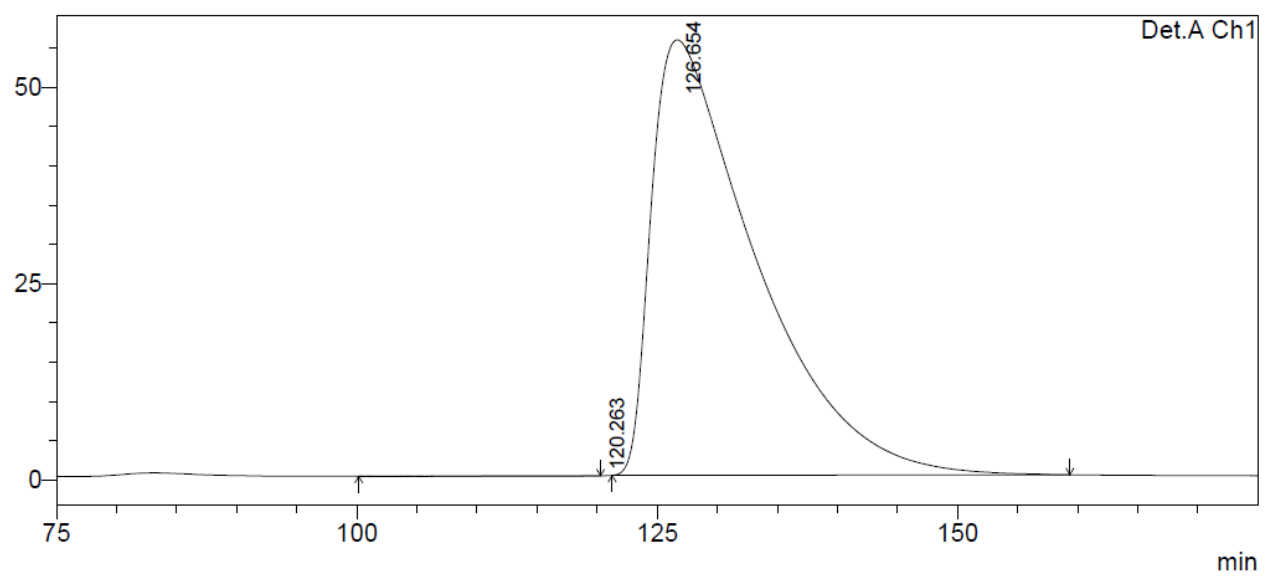

1 Det.A Ch $1 / 254 \mathrm{~nm}$

PeakTable

Detector A Ch1 $254 \mathrm{~nm}$
\begin{tabular}{|r|r|r|r|r|r|}
\hline Peak\# & Ret. Time & Area & Height & Area $\%$ & Height $\%$ \\
\hline 1 & 120.263 & -20159 & 1 & -0.060 & 0.002 \\
\hline 2 & 126.654 & 33548597 & 55445 & 100.060 & 99.998 \\
\hline Total & & 33528438 & 55446 & 100.000 & 100.000 \\
\hline
\end{tabular}


<smiles>O=C(C[C@H]1c2ccccc2[C@@H]2[C@@H](c3ccccc3)OC(=O)[C@H]12)c1ccccc1</smiles>

$11 \mathrm{a}$

HPLC date of compound 11a: AD-H column, 80:20 hexane : IPA, flow rate: $1.0 \mathrm{ml} / \mathrm{min}, 254 \mathrm{~nm}, 25^{\circ} \mathrm{C},>99 \%$ ee.

$\mathrm{mV}$

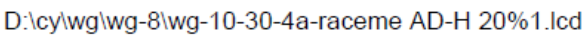

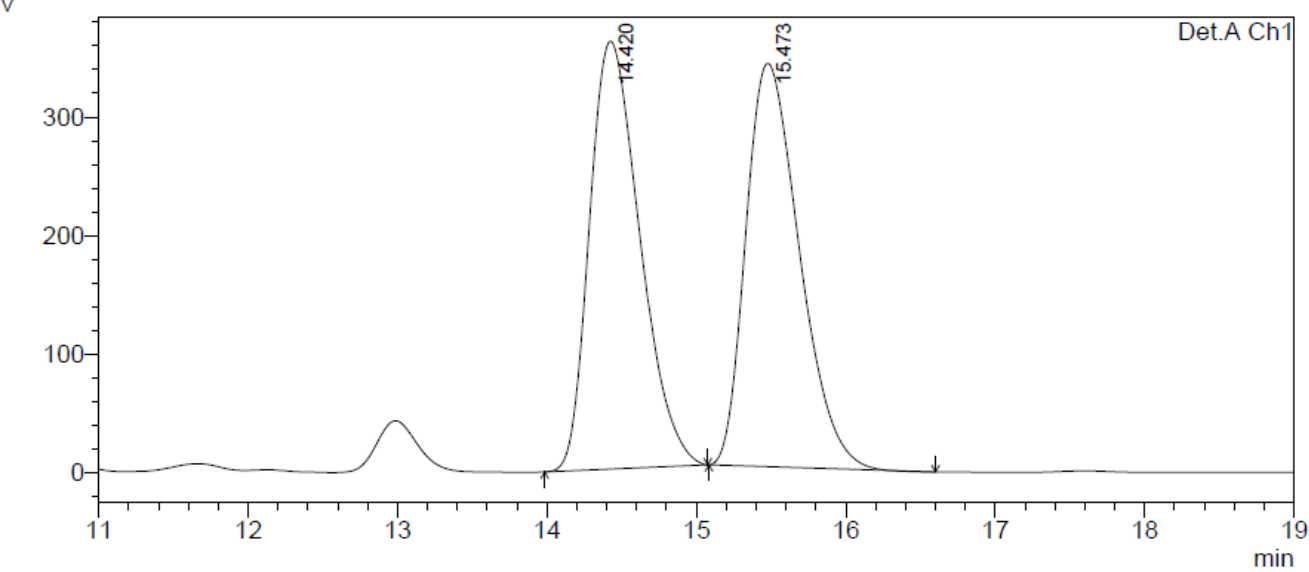

1 Det.A Ch1/254nm

DeakTable
\begin{tabular}{|r|r|r|r|r|r|}
\hline \multicolumn{1}{|c|}{ Peak\# } & Ret. Time & \multicolumn{1}{c|}{ Area } & Height & \multicolumn{1}{c|}{ Area \% } & \multicolumn{1}{c|}{ Height \% } \\
\hline 1 & 14.420 & 8456524 & 360810 & 49.960 & 51.477 \\
\hline 2 & 15.473 & 8470220 & 340101 & 50.040 & 48.523 \\
\hline Total & & 16926744 & 700910 & 100.000 & 100.000 \\
\hline
\end{tabular}

$\mathrm{mV}$

D:lcylwglwg-8|wg-10-30-4a AD-H 20\%1.Icd

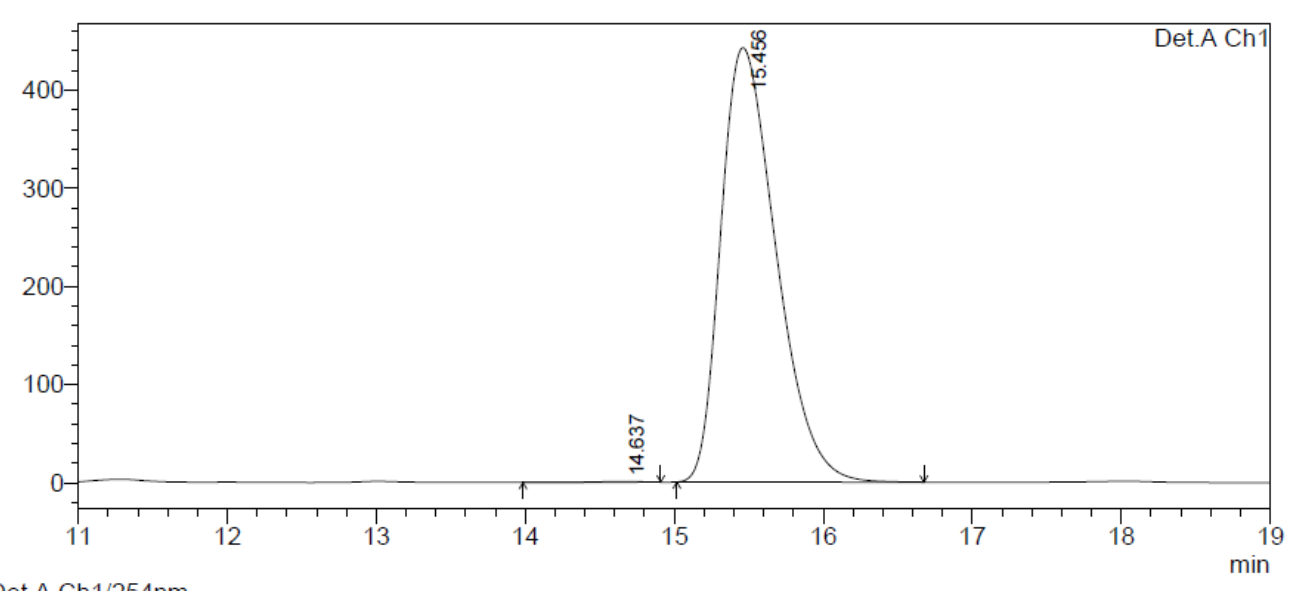

1 Det.A Ch1/254nm

PeakTable

Detector A Ch1 $254 \mathrm{~nm}$
\begin{tabular}{|r|r|r|r|r|r|}
\hline Peak\# & Ret. Time & Area & Height & Area $\%$ & \multicolumn{1}{|c|}{ Height $\%$} \\
\hline 1 & 14.637 & 12265 & 711 & 0.107 & 0.160 \\
\hline 2 & 15.456 & 11423662 & 442452 & 99.893 & 99.840 \\
\hline Total & & 11435927 & 443163 & 100.000 & 100.000 \\
\hline
\end{tabular}


<smiles>CCc1ccccc1[C@@H]1OC(=O)[C@@H]2[C@H](CC(=O)c3ccccc3)c3ccccc3[C@H]12</smiles>

11b

HPLC date of compound 11b: AD-H column, 80:20 hexane : IPA, flow rate: $1.0 \mathrm{ml} / \mathrm{min}, 254 \mathrm{~nm}, 25^{\circ} \mathrm{C},>99 \%$ ee.

$\mathrm{mV}$

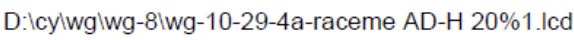

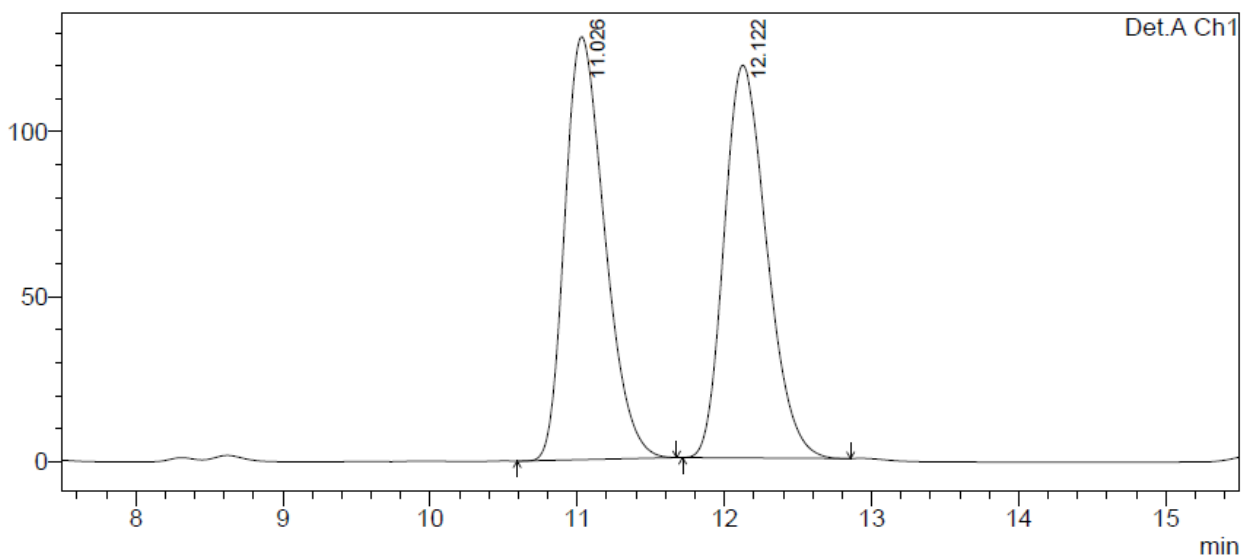

1 Det.A Ch1/254nm

PeakTable
Detector A Ch1 254nm
\begin{tabular}{|r|r|r|r|r|r|}
\hline Peak\# & Ret. Time & Area & Height & \multicolumn{1}{c|}{ Area \% } & \multicolumn{1}{|c|}{ Height \% } \\
\hline 1 & 11.026 & 2420927 & 128127 & 50.148 & 51.842 \\
\hline 2 & 12.122 & 2406663 & 119020 & 49.852 & 48.158 \\
\hline Total & & 4827591 & 247147 & 100.000 & 100.000 \\
\hline
\end{tabular}

$\mathrm{mV}$

D:Icylwglwg-8lwg-10-29-4a AD-H 20\%1.Icd

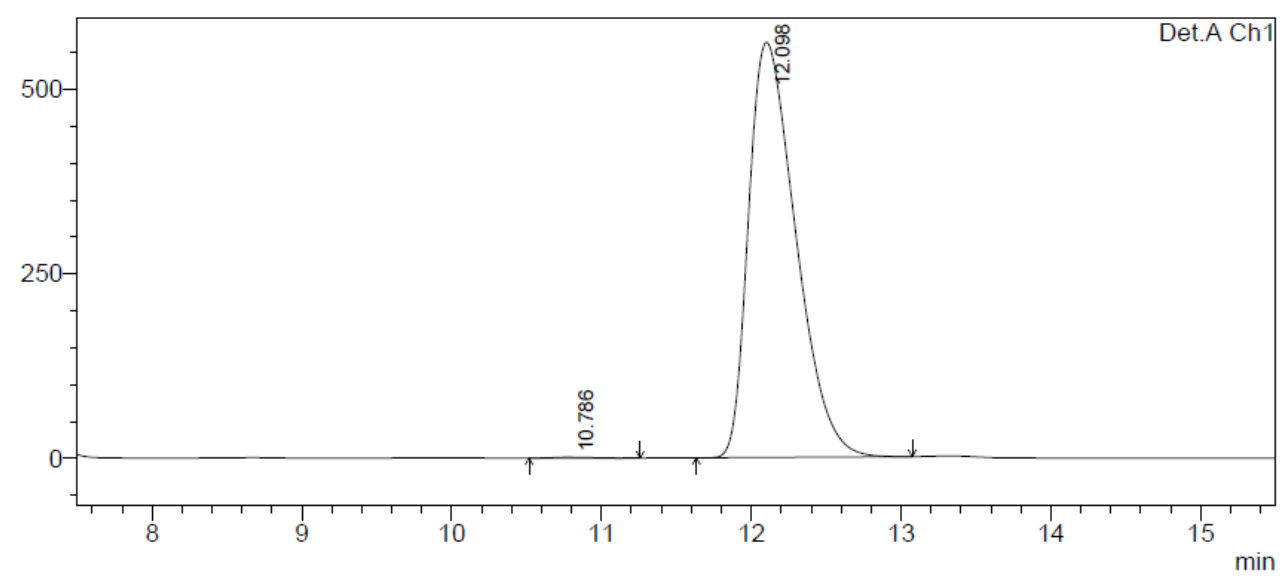

1 Det.A Ch1/254nm

PeakTable

Detector A Ch1 $254 \mathrm{~nm}$
\begin{tabular}{|r|r|r|r|r|r|}
\hline Peak\# & Ret. Time & \multicolumn{1}{c|}{ Area } & Height & Area $\%$ & \multicolumn{1}{c|}{ Height $\%$} \\
\hline 1 & 10.786 & 18407 & 1289 & 0.149 & 0.228 \\
\hline 2 & 12.098 & 12338875 & 563008 & 99.851 & 99.772 \\
\hline Total & & 12357283 & 564297 & 100.000 & 100.000 \\
\hline
\end{tabular}


<smiles>COc1ccccc1[C@@H]1OC(=O)[C@H]2[C@H](CC(=O)c3ccccc3)c3ccccc3[C@@H]21</smiles>

$11 \mathrm{c}$

HPLC date of compound 11c: OD-H column, $80: 20$ hexane : IPA, flow rate: $1.0 \mathrm{ml} / \mathrm{min}, 254 \mathrm{~nm}, 25^{\circ} \mathrm{C}, 99 \%$ ee.

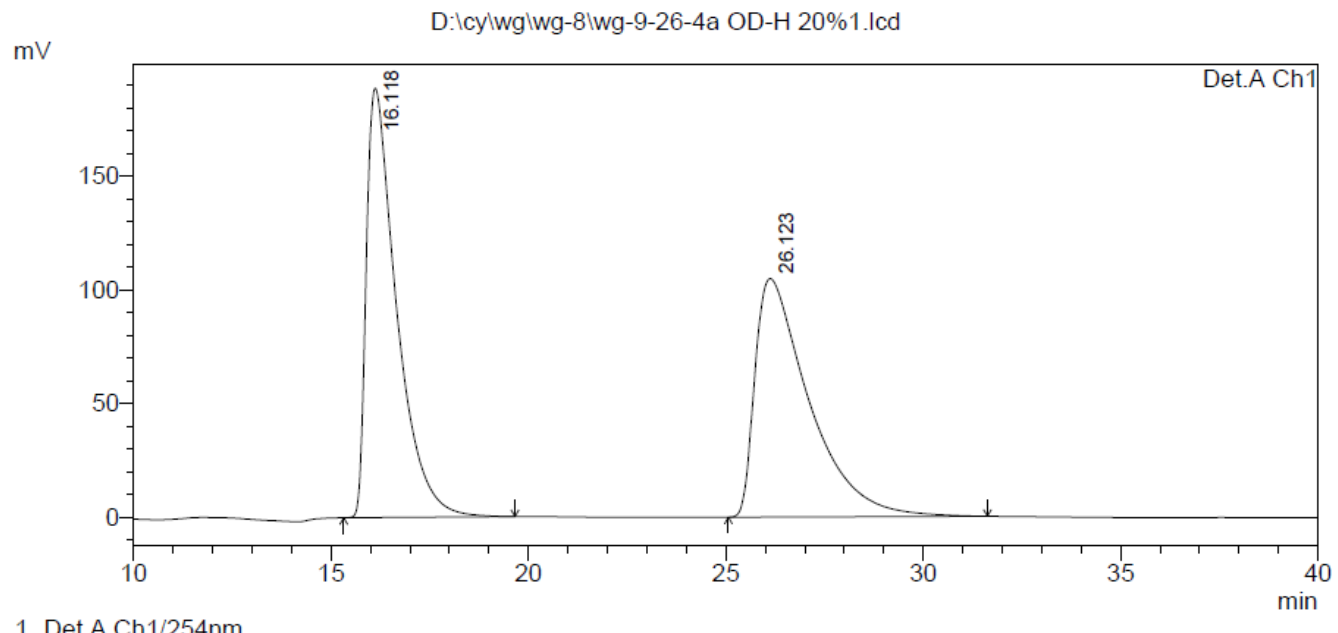

1 Det.A Ch1/254nm

PeakTable

Detector A Ch1 $254 \mathrm{~nm}$
\begin{tabular}{|r|r|r|r|r|r|}
\hline Peak\# & Ret. Time & \multicolumn{1}{c|}{ Area } & Height & \multicolumn{1}{c|}{ Area $\%$} & \multicolumn{1}{c|}{ Height $\%$} \\
\hline 1 & 16.118 & 10052728 & 188621 & 50.162 & 64.284 \\
\hline 2 & 26.123 & 9987604 & 104798 & 49.838 & 35.716 \\
\hline Total & & 20040331 & 293419 & 100.000 & 100.000 \\
\hline
\end{tabular}

$\mathrm{mV}$

D:Icylwglwg-8|wg-10-20-4a OD-H 20\%1.Icd

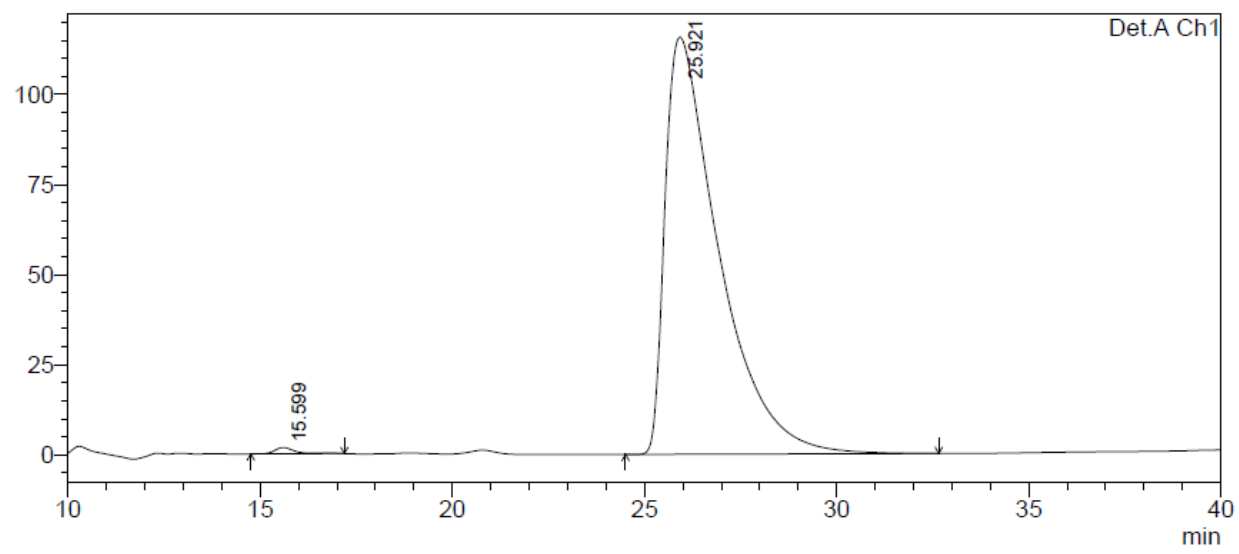

1 Det.A Ch1/254nm

Detector A Ch1 254nm
\begin{tabular}{|r|r|r|r|r|r|}
\hline Peak\# & Ret. Time & Area & \multicolumn{1}{c|}{ Height } & Area \% & \multicolumn{1}{c|}{ Height $\%$} \\
\hline 1 & 15.599 & 65229 & 1733 & 0.578 & 1.476 \\
\hline 2 & 25.921 & 11220676 & 115744 & 99.422 & 98.524 \\
\hline Total & & 11285905 & 117477 & 100.000 & 100.000 \\
\hline
\end{tabular}


<smiles>COc1cccc([C@@H]2OC(=O)[C@H]3[C@H](CC(=O)c4ccccc4)c4ccccc4[C@H]32)c1</smiles>

$11 d$

HPLC date of compound 11d: OD-H column, $80: 20$ hexane : IPA, flow rate: $1.0 \mathrm{ml} / \mathrm{min}, 254 \mathrm{~nm}, 25^{\circ} \mathrm{C}, 97 \%$ ee.

$\mathrm{mV}$

D:lcylwg $|w g-8| w g-9-27-4 a$ OD-H 20\%1.Icd

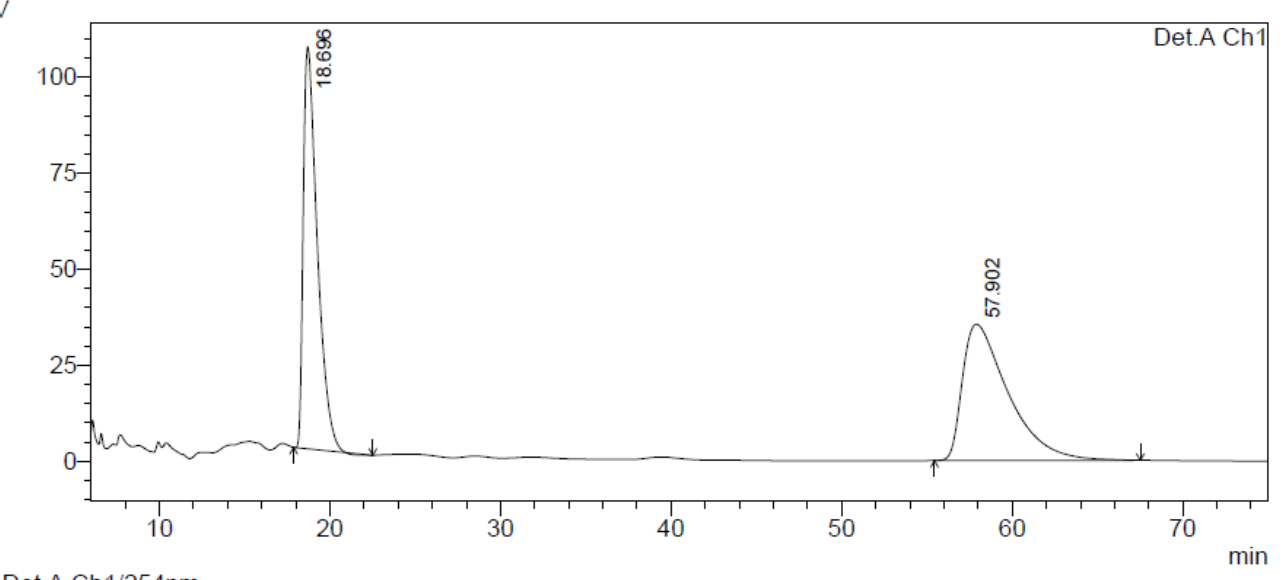

1 Det.A Ch $1 / 254 \mathrm{~nm}$

PeakTable

Detector A Ch1 $254 \mathrm{~nm}$
\begin{tabular}{|r|r|r|r|r|r|}
\hline Peak\# & Ret. Time & \multicolumn{1}{c|}{ Area } & Height & \multicolumn{1}{c|}{ Area \% } & \multicolumn{1}{c|}{ Height $\%$} \\
\hline 1 & 18.696 & 5980535 & 104727 & 47.479 & 74.682 \\
\hline 2 & 57.902 & 6615721 & 35504 & 52.521 & 25.318 \\
\hline Total & & 12596255 & 140231 & 100.000 & 100.000 \\
\hline
\end{tabular}

$\mathrm{mV}$

D:lcylwglwg-8lwg-10-21-4a OD-H 20\%1.Icd

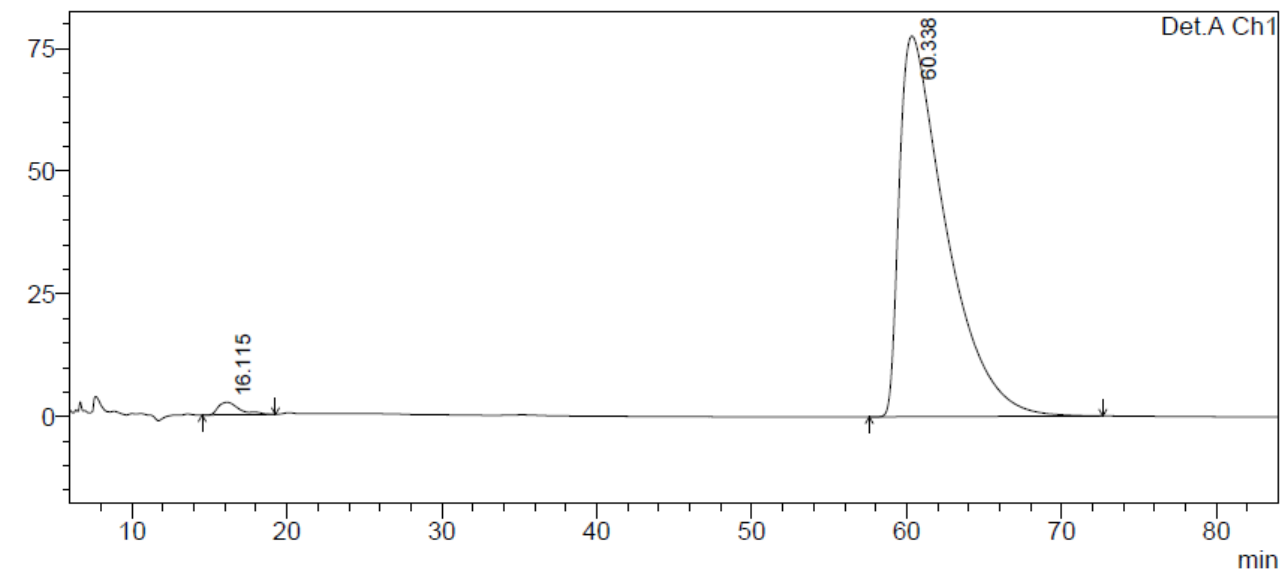

1 Det.A Ch1/254nm

PeakTable

Detector A Ch1 $254 \mathrm{~nm}$
\begin{tabular}{|r|r|r|r|r|r|}
\hline Peak\# & Ret. Time & \multicolumn{1}{c|}{ Area } & Height & Area $\%$ & Height $\%$ \\
\hline 1 & 16.115 & 244146 & 2548 & 1.477 & 3.182 \\
\hline 2 & 60.338 & 16281745 & 77541 & 98.523 & 96.818 \\
\hline Total & & 16525891 & 80089 & 100.000 & 100.000 \\
\hline
\end{tabular}


$\mathrm{MeO}$<smiles>O=C(C[C@H]1c2ccccc2[C@H]2[C@@H](c3ccc(I)cc3)OC(=O)[C@@H]21)c1ccccc1</smiles>

$11 \mathrm{e}$

HPLC date of compound 11e: AD-H column, 80:20 hexane : IPA, flow rate: $1.0 \mathrm{ml} / \mathrm{min}, 254 \mathrm{~nm}, 25^{\circ} \mathrm{C},>99 \%$ ee.

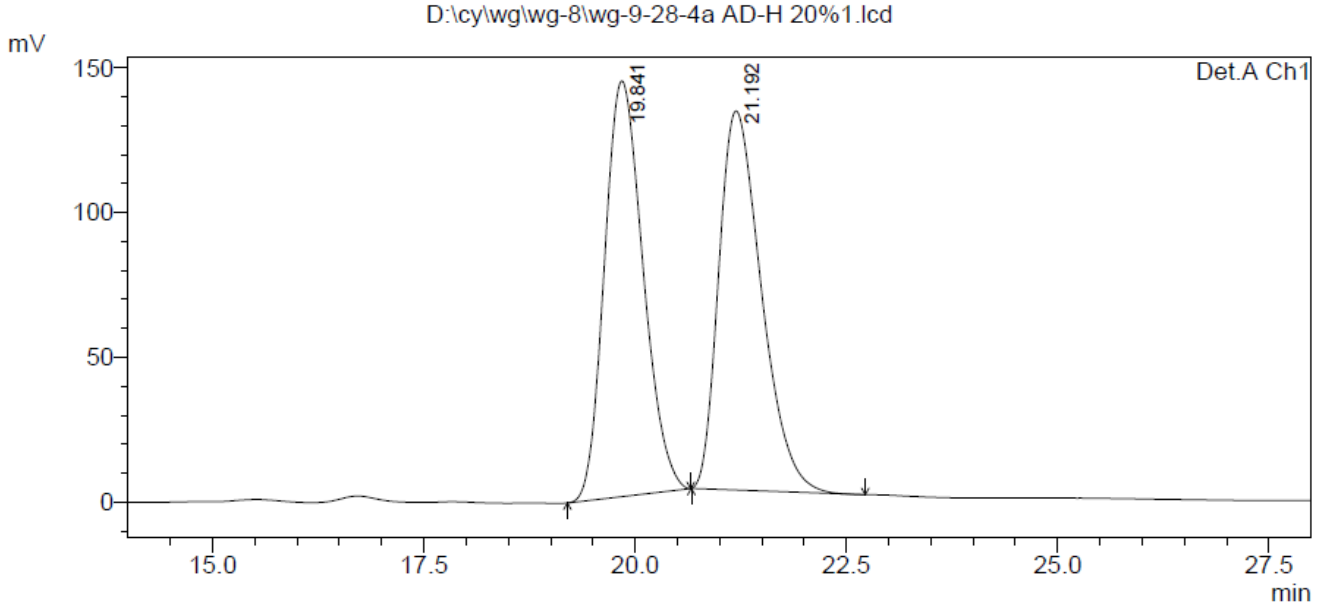

1 Det.A Ch $1 / 254 \mathrm{~nm}$

PeakTable

Detector A Ch1 $254 \mathrm{~nm}$
\begin{tabular}{|r|r|r|r|r|r|}
\hline Peak\# & Ret. Time & Area & Height & \multicolumn{1}{c|}{ Area $\%$} & \multicolumn{1}{c|}{ Height $\%$} \\
\hline 1 & 19.841 & 4556357 & 143583 & 49.855 & 52.307 \\
\hline 2 & 21.192 & 4582861 & 130920 & 50.145 & 47.693 \\
\hline Total & & 9139218 & 274503 & 100.000 & 100.000 \\
\hline
\end{tabular}

$\mathrm{mV}$

D:lcylwglwg-8lwg-10-22-4a AD-H 20\%1.Icd

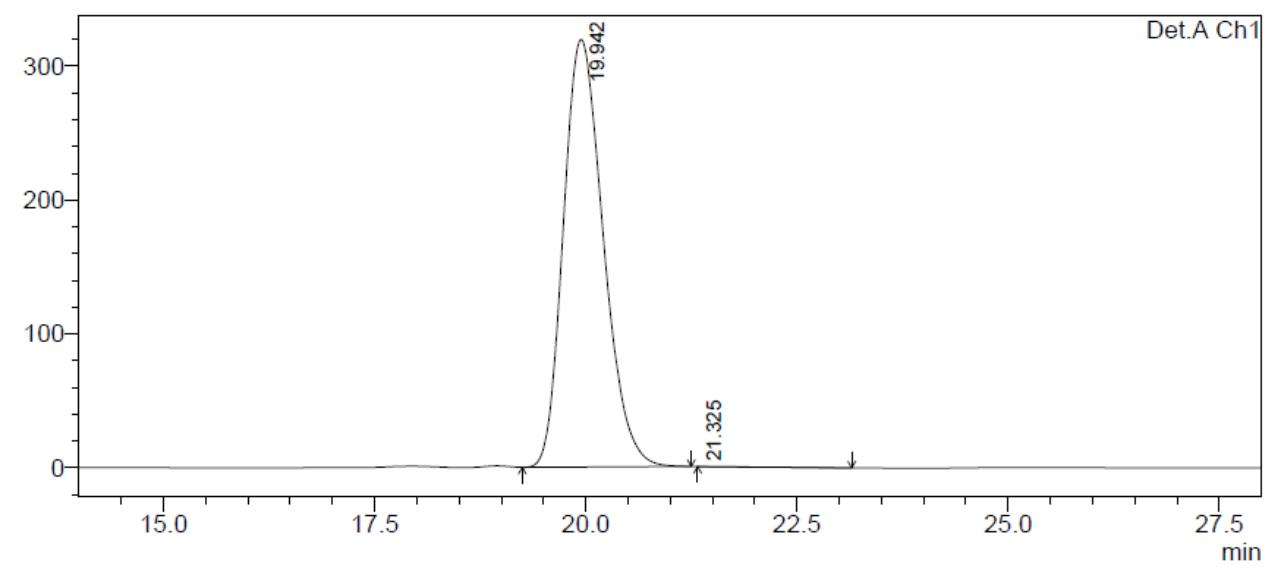

1 Det.A Ch1/254nm

PeakTable

Detector A Ch1 $254 \mathrm{~nm}$
\begin{tabular}{|r|r|r|r|r|r|}
\hline Peak\# & Ret. Time & Area & \multicolumn{1}{c|}{ Height } & \multicolumn{1}{c|}{ Area $\%$} & \multicolumn{1}{c|}{ Height $\%$} \\
\hline 1 & 19.942 & 10488626 & 319161 & 100.055 & 100.000 \\
\hline 2 & 21.325 & -5776 & 0 & -0.055 & 0.000 \\
\hline Total & & 10482850 & 319161 & 100.000 & 100.000 \\
\hline
\end{tabular}


<smiles>O=C(C[C@H]1c2ccccc2[C@@H]2[C@@H](c3ccccc3Br)OC(=O)[C@H]12)c1ccccc1</smiles>

$11 f$

HPLC date of compound 11f: OD-H column, 80:20 hexane : IPA, flow rate: $1.0 \mathrm{ml} / \mathrm{min}, 254 \mathrm{~nm}, 25^{\circ} \mathrm{C},>99 \%$ ee.

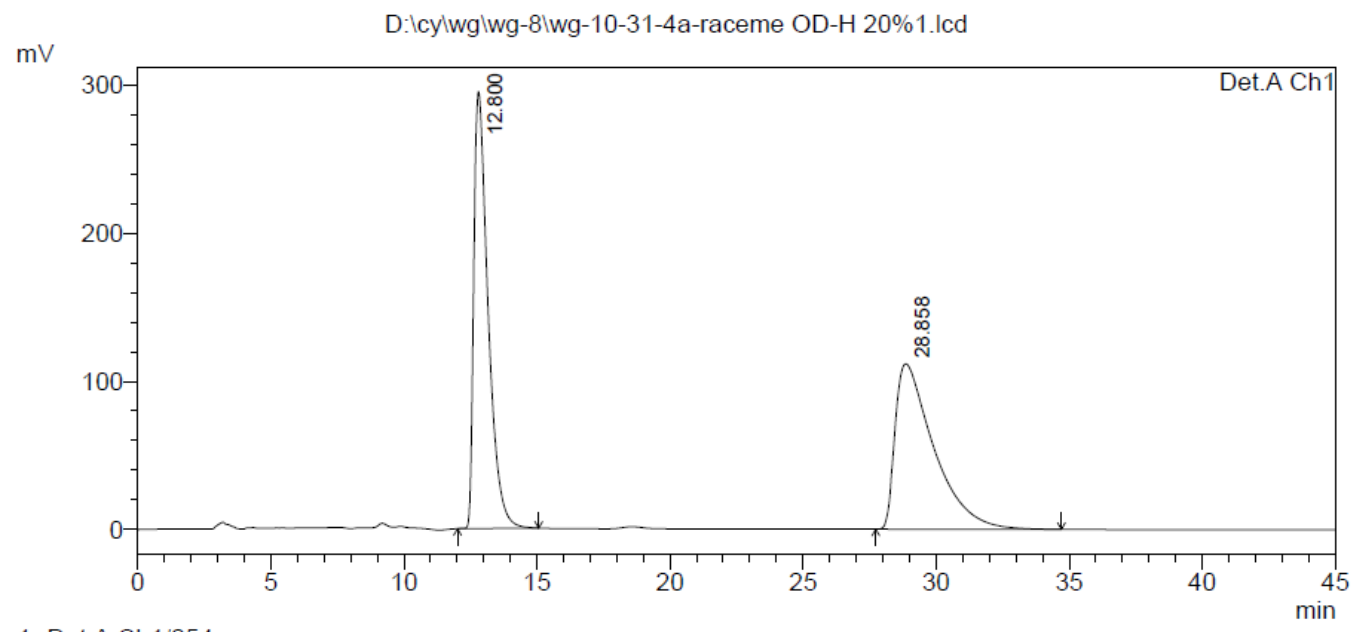

1 Det.A Ch1/254nm

PeakTable
\begin{tabular}{|r|r|r|r|r|r|}
\hline Detector A Ch1 254nm \\
\hline Peak\# & Ret. Time & Area & Height & \multicolumn{1}{c|}{ Area \% } & \multicolumn{1}{c|}{ Height \% } \\
\hline 1 & 12.800 & 11016133 & 295118 & 49.336 & 72.541 \\
\hline 2 & 28.858 & 11312467 & 111714 & 50.664 & 27.459 \\
\hline Total & & 22328600 & 406832 & 100.000 & 100.000 \\
\hline
\end{tabular}

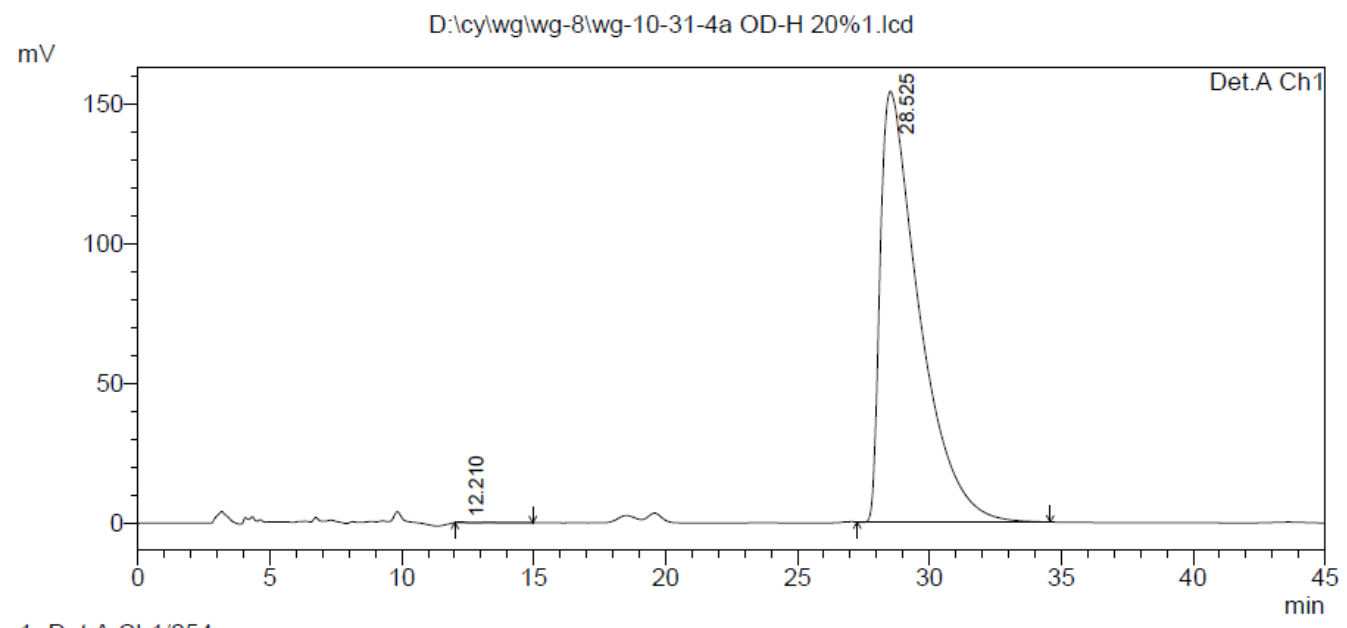

1 Det.A Ch1/254nm

PeakTable
\begin{tabular}{|r|r|r|r|r|r|}
\hline Detector A Ch1 254nm \\
\hline Peak\# & Ret. Time & Area & \multicolumn{1}{c|}{ Height } & \multicolumn{1}{c|}{ Area \% } & \multicolumn{1}{c|}{ Height \% } \\
\hline 1 & 12.210 & 6222 & 241 & 0.039 & 0.156 \\
\hline 2 & 28.525 & 15775507 & 154121 & 99.961 & 99.844 \\
\hline Total & & 15781729 & 154362 & 100.000 & 100.000 \\
\hline
\end{tabular}


<smiles>O=C(C[C@H]1c2ccccc2[C@@H]2[C@@H](c3ccccc3C(F)(F)F)OC(=O)[C@H]12)c1ccccc1</smiles>

$11 \mathrm{~g}$

HPLC date of compound 11g: OD-H column, 80:20 hexane : IPA, flow rate: $1.0 \mathrm{ml} / \mathrm{min}, 254 \mathrm{~nm}, 25^{\circ} \mathrm{C}, 99 \%$ ee.

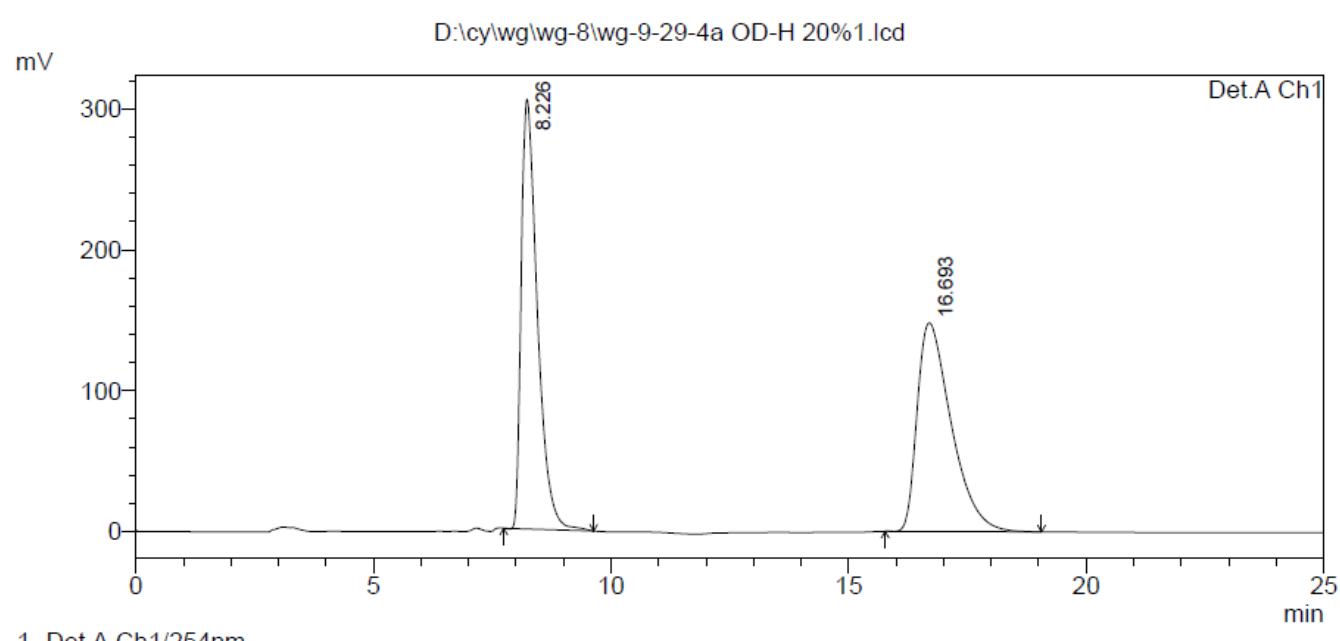

1 Det.A Ch1/254nm

PeakTable
Detector A Ch1 254nm
\begin{tabular}{|r|r|r|r|r|r|}
\hline Peak\# & Ret. Time & \multicolumn{1}{c|}{ Area } & Height & Area \% & \multicolumn{1}{c|}{ Height \% } \\
\hline 1 & 8.226 & 7229336 & 304979 & 49.445 & 67.316 \\
\hline 2 & 16.693 & 7391557 & 148077 & 50.555 & 32.684 \\
\hline Total & & 14620893 & 453056 & 100.000 & 100.000 \\
\hline
\end{tabular}

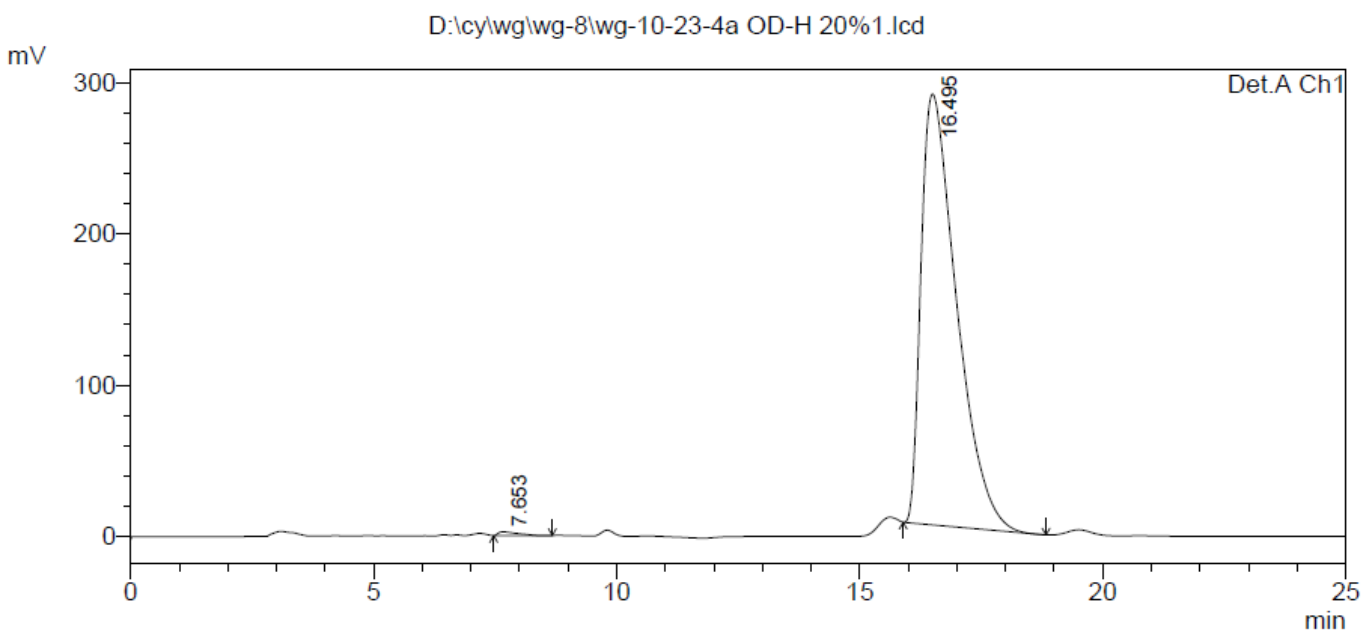

1 Det.A Ch1/254nm

PeakTable

Detector A Ch1 $254 \mathrm{~nm}$
\begin{tabular}{|r|r|r|r|r|r|}
\hline Peak\# & Ret. Time & \multicolumn{1}{c|}{ Area } & \multicolumn{1}{c|}{ Height } & Area $\%$ & \multicolumn{1}{c|}{ Height $\%$} \\
\hline 1 & 7.653 & 65214 & 2417 & 0.449 & 0.842 \\
\hline 2 & 16.495 & 14464962 & 284620 & 99.551 & 99.158 \\
\hline Total & & 14530176 & 287036 & 100.000 & 100.000 \\
\hline
\end{tabular}


<smiles>O=C(C[C@H]1c2ccccc2[C@H]2[C@@H](c3cccc(C(F)(F)F)c3)OC(=O)[C@@H]21)c1ccccc1</smiles>

$11 \mathrm{~h}$

HPLC date of compound 11h: AD-H column, 80:20 hexane : IPA, flow rate: $1.0 \mathrm{ml} / \mathrm{min}, 254 \mathrm{~nm}, 25^{\circ} \mathrm{C}, 94 \%$ ee

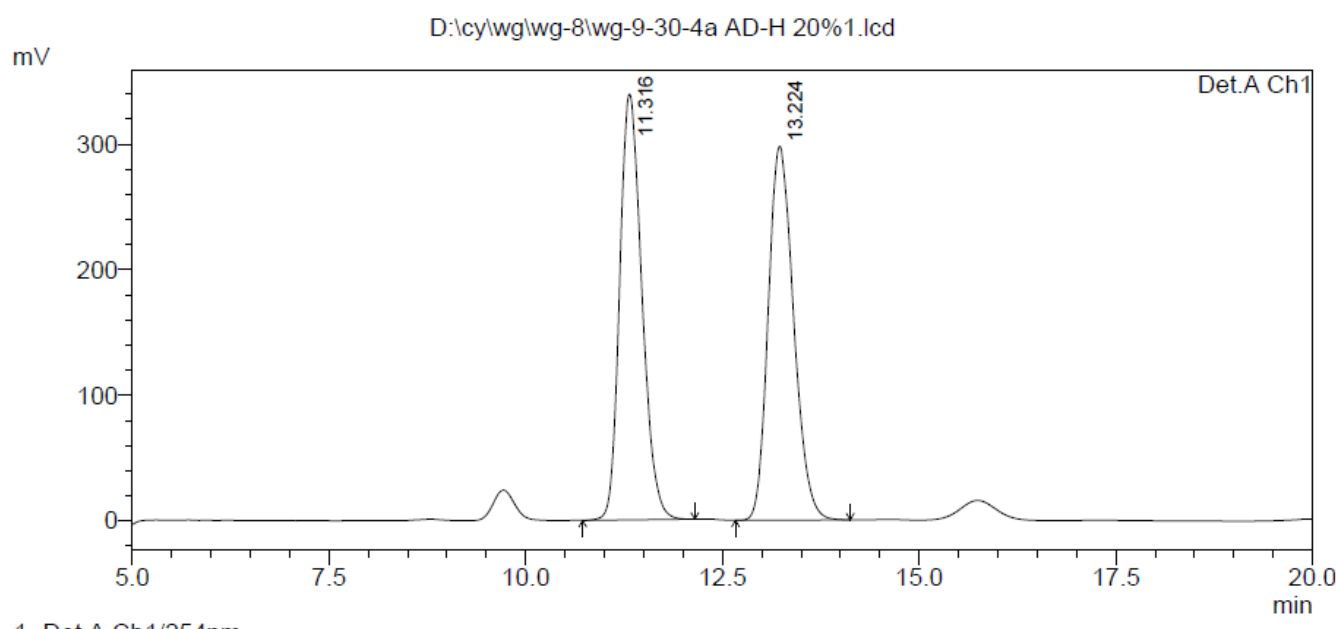

1 Det.A Ch1/254nm

Detector A Ch1 254nm
\begin{tabular}{|r|r|r|r|r|r|}
\hline Peak\# & Ret. Time & \multicolumn{1}{c|}{ Area } & Height & Area \% & \multicolumn{1}{c|}{ Height \% } \\
\hline 1 & 11.316 & 6664943 & 339371 & 49.868 & 53.256 \\
\hline 2 & 13.224 & 6700140 & 297879 & 50.132 & 46.744 \\
\hline Total & & 13365083 & 637250 & 100.000 & 100.000 \\
\hline
\end{tabular}

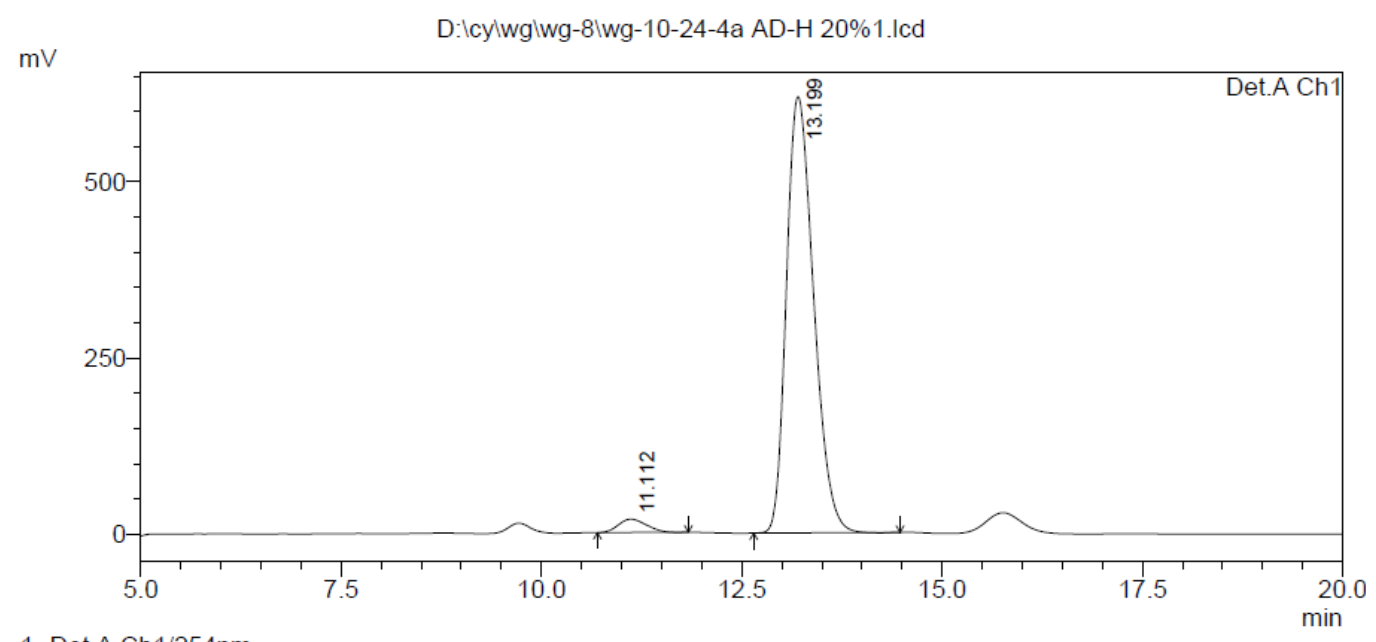

1 Det.A Ch1/254nm

PeakTable

Detector A Ch1 $254 \mathrm{~nm}$
\begin{tabular}{|r|r|r|r|r|r|}
\hline Peak\# & Ret. Time & \multicolumn{1}{c|}{ Area } & Height & Area \% & \multicolumn{1}{c|}{ Height $\%$} \\
\hline 1 & 11.112 & 449833 & 18802 & 2.987 & 2.948 \\
\hline 2 & 13.199 & 14609145 & 619021 & 97.013 & 97.052 \\
\hline Total & & 15058978 & 637823 & 100.000 & 100.000 \\
\hline
\end{tabular}


<smiles>O=C(C[C@H]1c2ccccc2[C@@H]2[C@@H](c3ccc(C(F)(F)F)cc3)OC(=O)[C@H]12)c1ccccc1</smiles>

$11 \mathrm{i}$

HPLC date of compound 11i: AD-H column, 80:20 hexane : IPA, flow rate: $1.0 \mathrm{ml} / \mathrm{min}, 254 \mathrm{~nm}, 25^{\circ} \mathrm{C}, 99 \%$ ee.

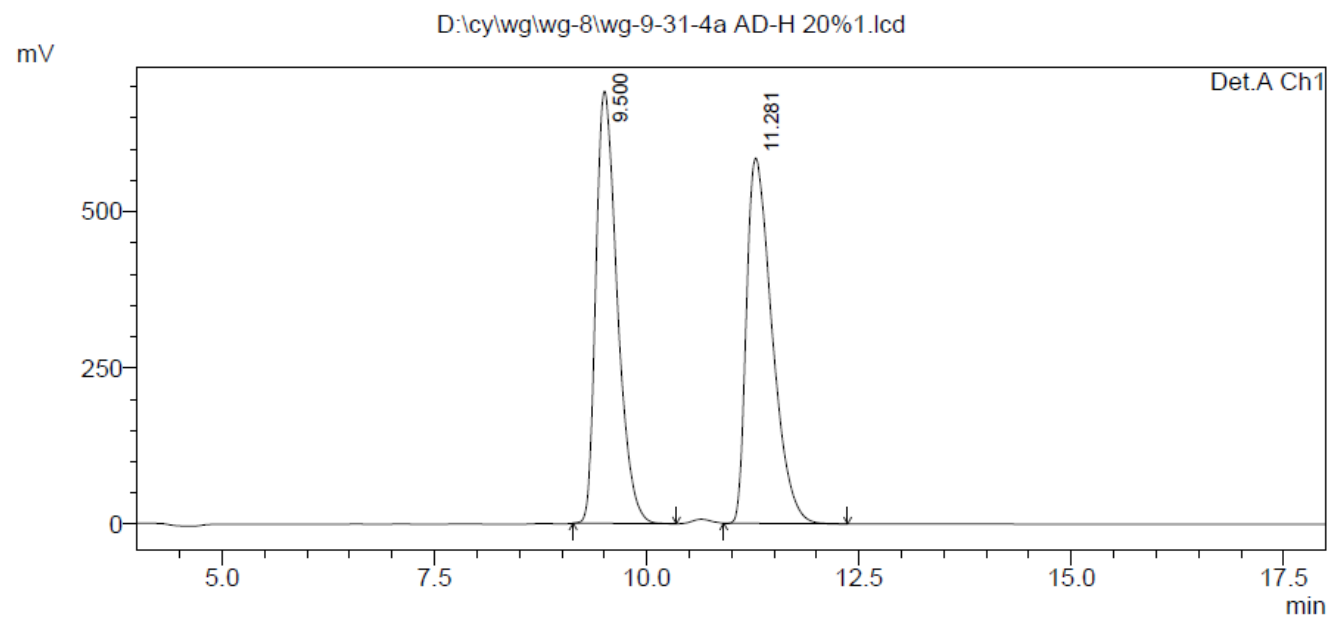

1 Det.A Ch1/254nm

Detector A Ch1 254nm
\begin{tabular}{|r|r|r|r|r|r|}
\hline Peak\# & Ret. Time & Area & \multicolumn{1}{c|}{ Height } & Area $\%$ & \multicolumn{1}{|c|}{ Height \% } \\
\hline 1 & 9.500 & 12085389 & 690887 & 49.634 & 54.186 \\
\hline 2 & 11.281 & 12263389 & 584148 & 50.366 & 45.814 \\
\hline Total & & 24348778 & 1275035 & 100.000 & 100.000 \\
\hline
\end{tabular}

$\mathrm{mV}$

D:Icylwglwg-8lwg-10-25-4a AD-H 20\%1.Icd

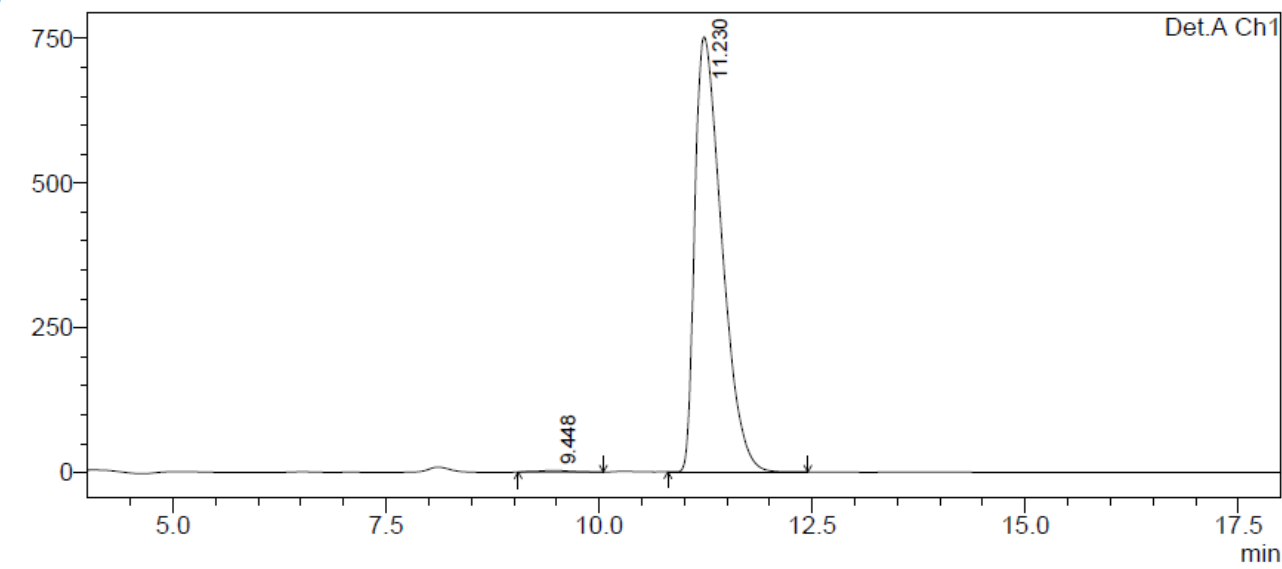

1 Det.A Ch1/254nm

PeakTable

Detector A Ch1 $254 \mathrm{~nm}$
\begin{tabular}{|r|r|r|r|r|r|}
\hline Peak\# & Ret. Time & Area & Height & Area $\%$ & \multicolumn{1}{c|}{ Height $\%$} \\
\hline 1 & 9.448 & 69123 & 2579 & 0.415 & 0.342 \\
\hline 2 & 11.230 & 16567581 & 751940 & 99.585 & 99.658 \\
\hline Total & & 16636704 & 754519 & 100.000 & 100.000 \\
\hline
\end{tabular}




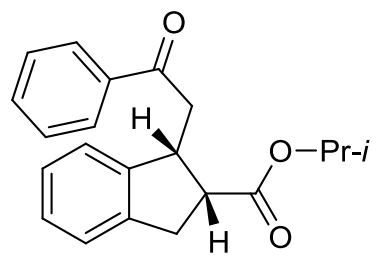

6a

HPLC date of compound 6a: AD-H column, 95:5 hexane : IPA, flow rate: $1.0 \mathrm{ml} / \mathrm{min}, 254 \mathrm{~nm}, 25^{\circ} \mathrm{C}, 95 \%$ ee.

$\mathrm{mV}$ D:Icylwglwg-8lwg-9-10-a-raceme AD-H 5\%1.Icd

$\mathrm{mV}$

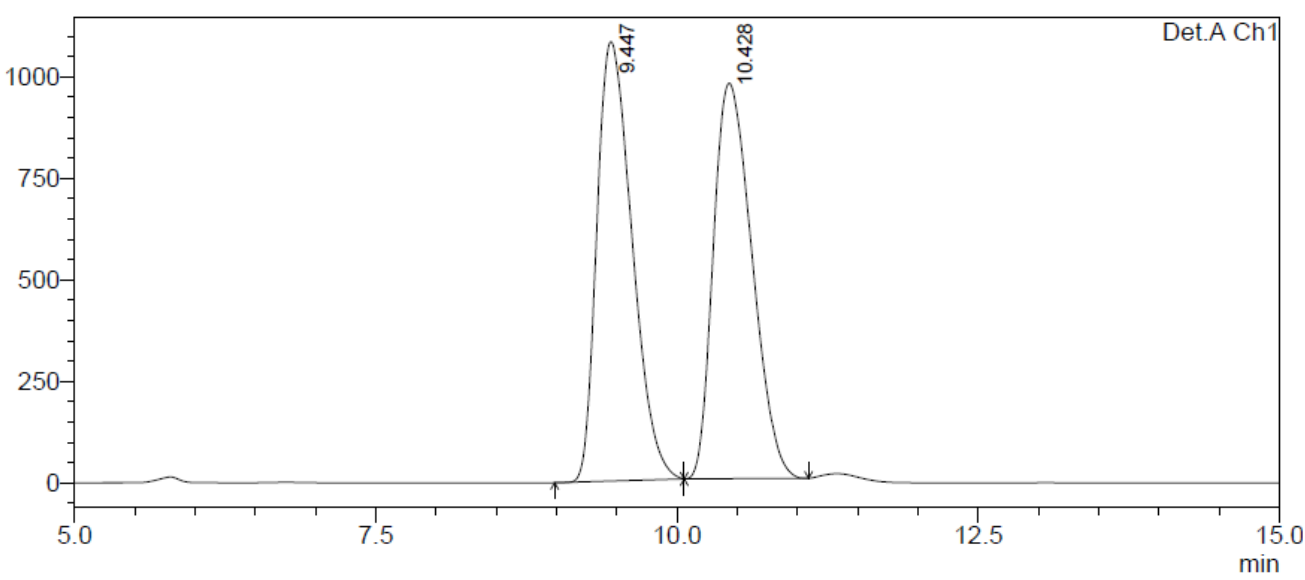

1 Det.A Ch $1 / 254 \mathrm{~nm}$

Detector A Ch1 254nm
\begin{tabular}{|r|r|r|r|r|r|}
\hline Peak\# & Ret. Time & \multicolumn{1}{l}{ Area } & \multicolumn{1}{c|}{ Height } & Area $\%$ & \multicolumn{1}{c|}{ Height $\%$} \\
\hline 1 & 9.447 & 21961013 & 1081348 & 50.308 & 52.628 \\
\hline 2 & 10.428 & 21691888 & 973341 & 49.692 & 47.372 \\
\hline Total & & 43652901 & 2054689 & 100.000 & 100.000 \\
\hline
\end{tabular}

$\mathrm{mV}$

D:Icylwglwg-8Iwg-9-10-a AD-H 5\%1.Icd

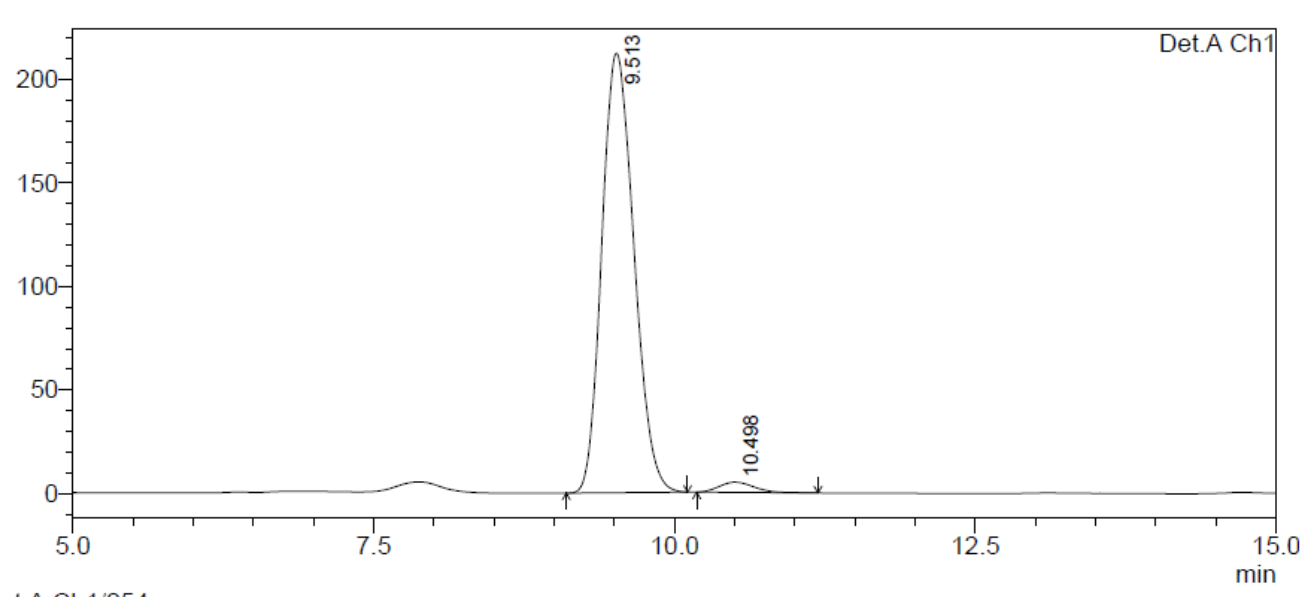

1 Det.A Ch1/254nm

PeakTable

Detector A Ch1 $254 \mathrm{~nm}$
\begin{tabular}{|r|r|r|r|r|r|}
\hline Peak\# & Ret. Time & \multicolumn{1}{c|}{ Area } & Height & \multicolumn{1}{c|}{ Area $\%$} & \multicolumn{1}{c|}{ Height $\%$} \\
\hline 1 & 9.513 & 3947058 & 212383 & 97.545 & 97.730 \\
\hline 2 & 10.498 & 99340 & 4933 & 2.455 & 2.270 \\
\hline Total & & 4046398 & 217317 & 100.000 & 100.000 \\
\hline
\end{tabular}


<smiles>CCOC(=O)C1Cc2ccccc2C1CC(=O)c1ccc(Br)cc1</smiles>

$6 \mathrm{~h}$

HPLC date of compound $6 \mathbf{h}$ : AD-H column, $95: 5$ hexane : IPA, flow rate: $1.0 \mathrm{ml} / \mathrm{min}, 254 \mathrm{~nm}, 25^{\circ} \mathrm{C}, 96 \%$ ee.

$\mathrm{mV}$ D: Icylwglwg-8lwg-10-7-a-raceme AD-H 5\%1.lcd

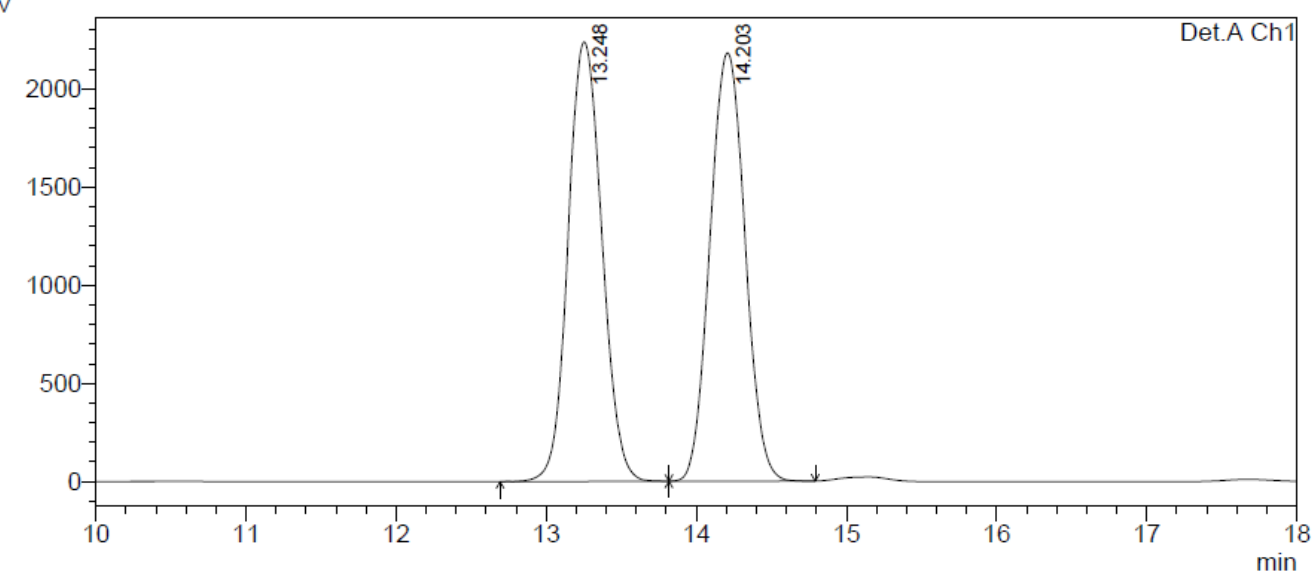

1 Det.A Ch1/254nm

PeakTable
\begin{tabular}{|r|r|r|r|r|r|}
\hline Peak\# & Ret. Time & Area & Height & Area $\%$ & \multicolumn{1}{c|}{ Height $\%$} \\
\hline 1 & 13.248 & 35144616 & 2239111 & 49.798 & 50.641 \\
\hline 2 & 14.203 & 35430401 & 2182395 & 50.202 & 49.359 \\
\hline Total & & 70575017 & 4421505 & 100.000 & 100.000 \\
\hline
\end{tabular}

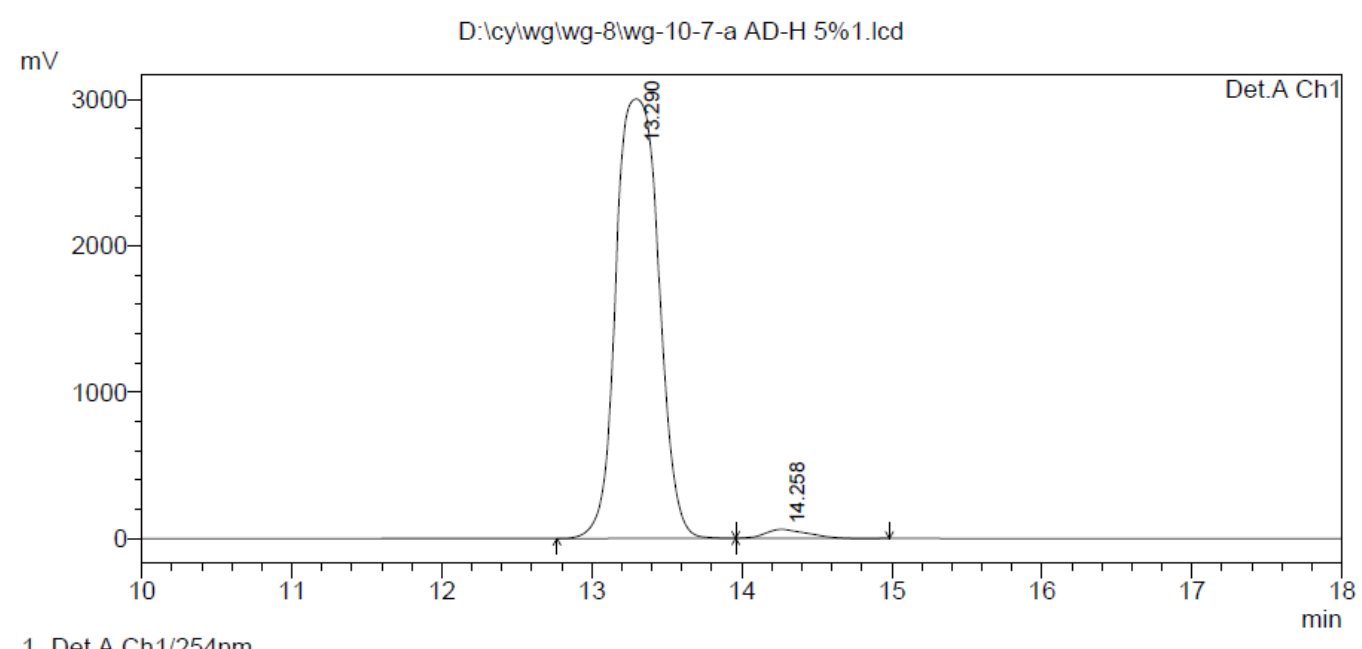

1 Det.A Ch1/254nm

Detector A Ch1 254nm
\begin{tabular}{|r|r|r|r|r|r|}
\hline Peak\# & Ret. Time & \multicolumn{1}{|c|}{ Area } & \multicolumn{1}{c|}{ Height } & Area \% & \multicolumn{1}{|c|}{ Height \% } \\
\hline 1 & 13.290 & 59622839 & 3004248 & 98.143 & 98.043 \\
\hline 2 & 14.258 & 1127933 & 59972 & 1.857 & 1.957 \\
\hline Total & & 60750772 & 3064220 & 100.000 & 100.000 \\
\hline
\end{tabular}

